\title{
Organische Petrologie und Mikrofazies der mitteleozänen Seesedimente des Eckfelder Maares (Südwesteifel)
}

\author{
Dissertation \\ zur Erlangung des Doktorgrades \\ der Mathematisch-Naturwissenschaftlichen Fakultäten \\ der Georg-August-Universität zu Göttingen
}

vorgelegt von

Volker Bullwinkel

aus Bremerhaven

Göttingen 2003 
D 7

Referent: $\quad$ Prof. Dr. W. Riegel

Korreferent: Prof. Dr. J. Paul

$\underline{\text { Tag der mündlichen Prüfung: }}$ 


\section{Kurzfassung}

In der vorliegenden Arbeit sind die Ergebnisse des von der Deutschen Forschungsgemeinschaft (DFG) geförderten Projektes "Organische Petrologie und Mikrofazies der mitteleozänen Seesedimente des Eckfelder Maares (Südwesteifel)" dokumentiert. Die Untersuchungen sind Bestandteil des interdisziplinär angelegten Gemeinschaftsprojektes "Bohrung Eckfelder Maar 1996" unter der Koordination des Naturhistorischen Museums Mainz / Landessammlung für Naturkunde Rheinland-Pfalz. Projektziel ist eine detaillierte Rekonstruktion des Paläoökosystems "Eckfelder Maarsee".

Anhand einer hochauflösenden Zonierung der beckenzentralen Bohrprofile Eckfeld 1/96, 2/96 und 3/96 in drei Lithofazieszonen (LFZ) mit insgesamt acht Mikrofazieszonen (MFZ) wird die Sedimentationsgeschichte des mitteleozänen Eckfeld-Sees nachvollzogen.

Eine quantitative Analyse des partikulären organischen Materials (POM) an 54 Kornschliffproben der Eckfelder Profundalsedimente zeigt vertikale Variationen im Verteilungsmuster von organischer und mineralischer Substanz auf. Die mittels statistischer Methoden (ClusterAnalyse) aufbereiteten Datensätze erlauben eine objektive Identifizierung übergeordneter Schwankungen im Verhältnis von Primärproduktion zu organo-detritischem Eintrag.

Auf der Grundlage von durchlicht-, auflicht-, fluoreszenz- und elektronenmikroskopischen Untersuchungen an insgesamt 74 Dünn- und Stückschliffen werden diskrete Laminen-Typen (LT) charakterisiert, die sich untereinander in ihrem Stoffbestand und ihrer diagenetischen Überprägung unterscheiden. Durch eine systematische Differenzierung von Mikroschichtungsgefügen können grundlegende Bildungs- und Ablagerungsmechanismen von organischer und mineralischer Substanz im Eckfelder Maar aufgezeigt werden. 


\begin{abstract}
The present thesis documents and discusses the results of the project "Organic petrology and microfacies of the Middle Eocene lacustrine sediments from the Eckfeld Maar (Southwest Eifel)" supported by the German Research Council (DFG). The investigations are part of the interdisciplinary cooperative project "Drill core Eckfeld Maar 1996" aimed at a detailed reconstruction of the "Eckfeld maar lake ecosystem" and coordinated by the Museum of Natural History Mainz / State Collection of Natural Sciences of Rhineland-Palatinate.
\end{abstract}

The reconstruction of the sedimentary history of the Middle Eocene Lake Eckfeld is based on three cores (Eckfeld 1/96, 2/96 and 3/96) drilled near the centre of the lake basin. The lake sediments are subdivided into three lithofacies zones (LFZ) and eight microfacies zones (MFZ).

The quantitative determination of particulate organic matter (POM) carried out on 54 polished sections of crushed samples reveals significant variations in the vertical distribution of organic and mineral components. Statistical tests (cluster analysis) of data sets allow the objective identification of fluctuations in the ratio of primary production to input of organic detritus.

Based on the study of 74 thin and polished sections of oriented block samples by means of transmitted light, fluorescence and scanning electron microscopy several distinctive types of laminae (LT) are recognized and described. They differ in particular with regard to their primary composition as well as to the degree of diagenetic alteration. By differentiating and interpreting sediment fabrics and bedding features on a microscale some fundamental mechanisms involved in the formation and deposition of organic and mineral components in the Eckfeld Maar can be recognized. 


\section{Inhalt}

\section{Abkürzungen}

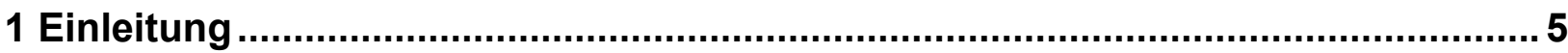

2 Das Eckfelder Maar ...................................................................................................... 6

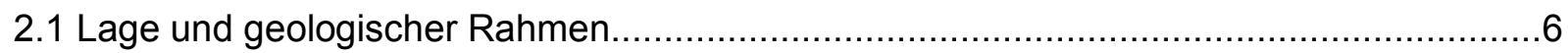

2.2 Klima und eozänes Relief im Raum Manderscheid/Eckfeld ..........................................

2.3 Erforschungsgeschichte und gegenwärtiger Kenntnisstand ...................................... 8

3 Material und Methoden .................................................................................. 13

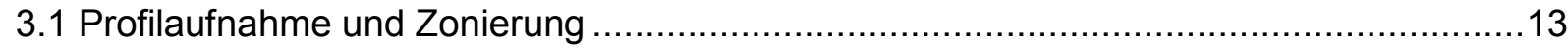

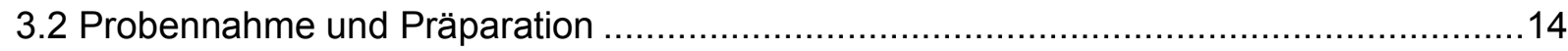

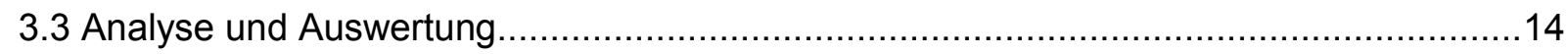

4 Vertikale Zonierung der zentralen Seesedimente............................................ 17

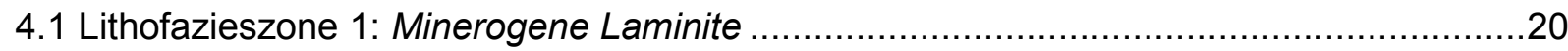

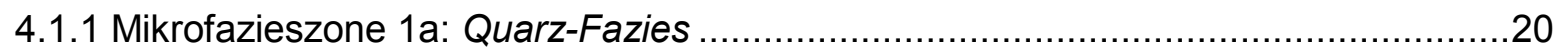

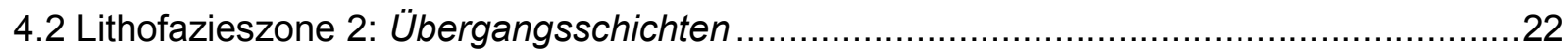

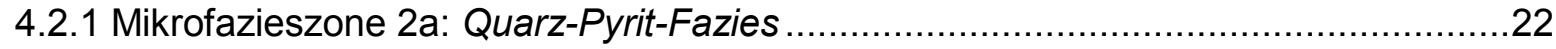

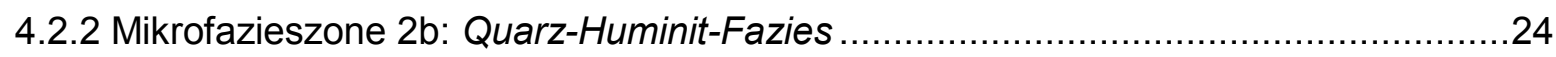

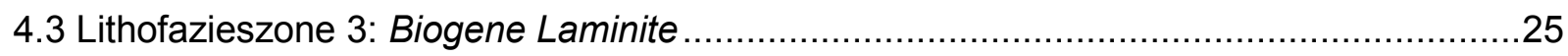

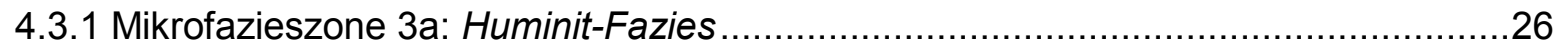

4.3.2 Mikrofazieszone 3b: Aulacoseira-Fazies ....................................................28

4.3.3 Mikrofazieszone 3c: Huminit-Opal-Fazies .......................................................... 30

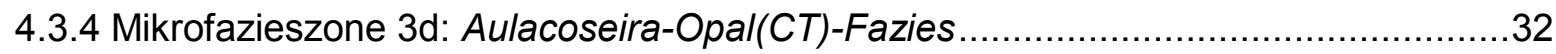

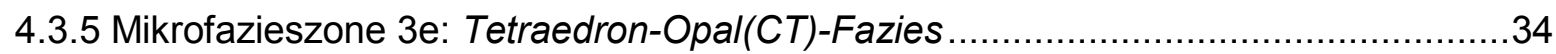

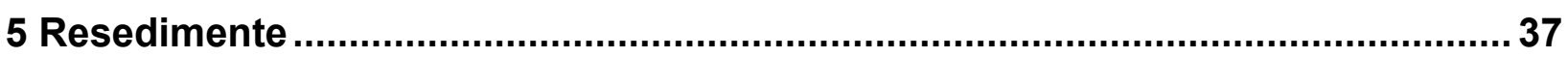

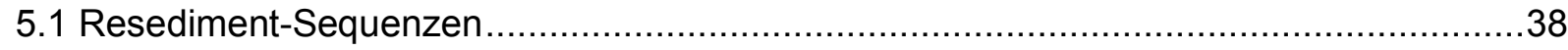

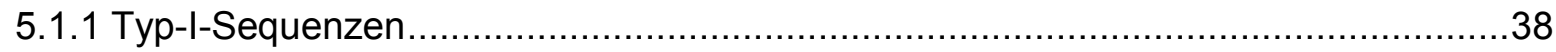

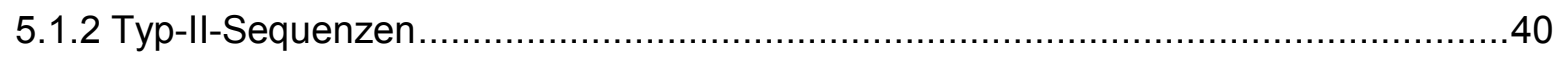

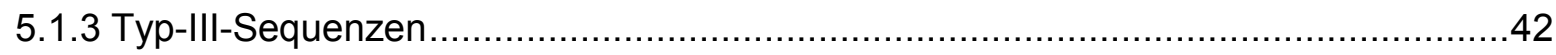

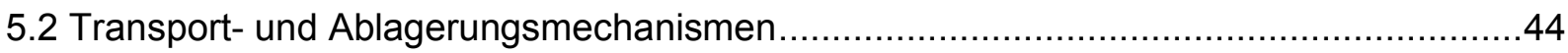

6 Bestandteile der zentralen Seesedimente........................................................49

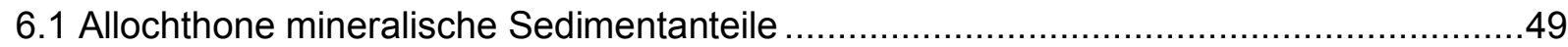

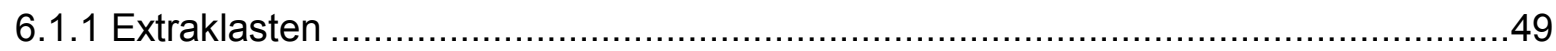

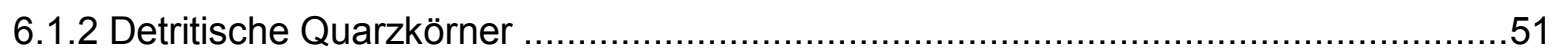

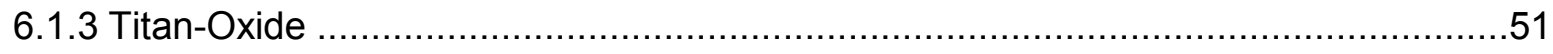

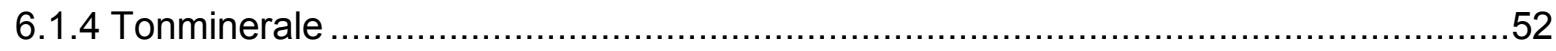

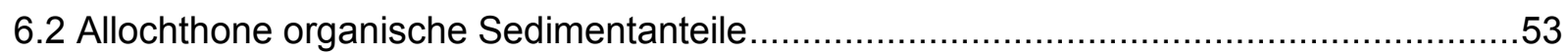

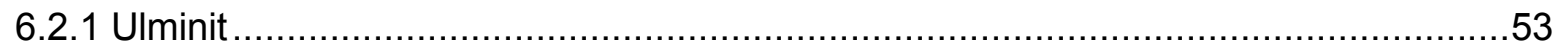

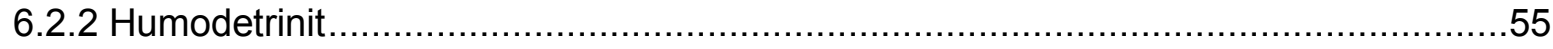

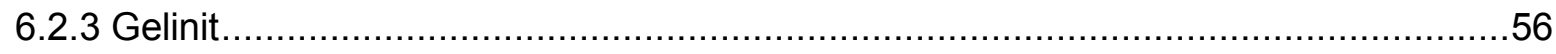

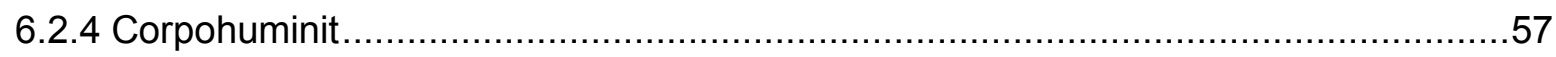

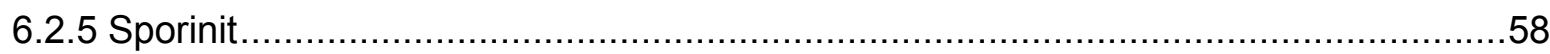

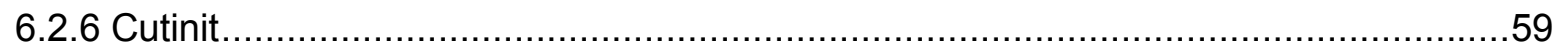

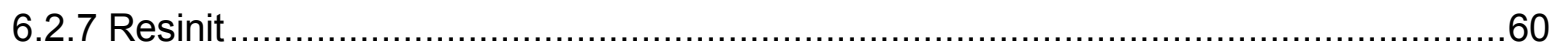




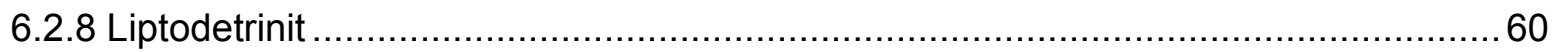

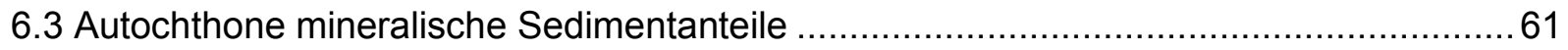

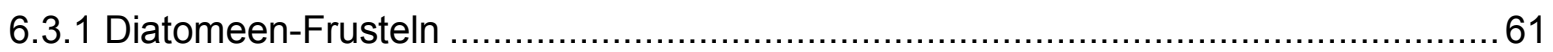

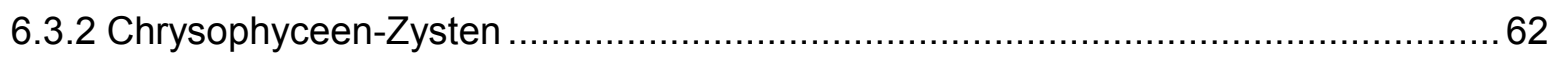

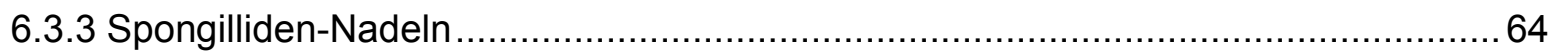

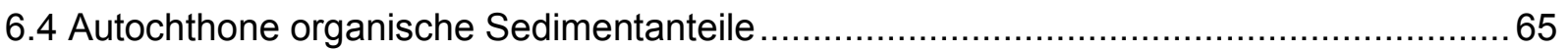

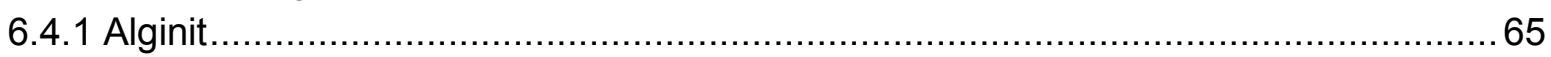

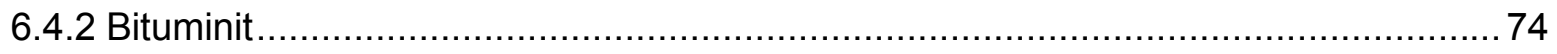

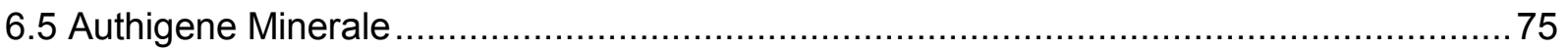

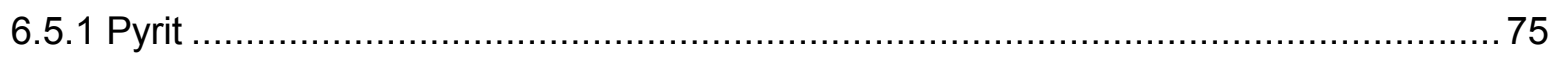

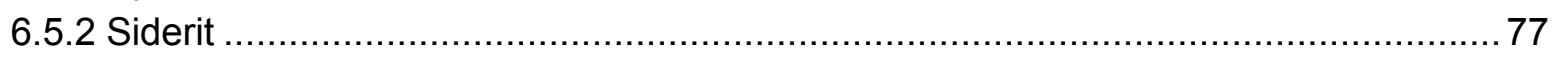

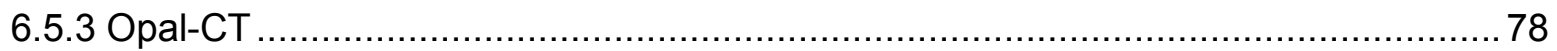

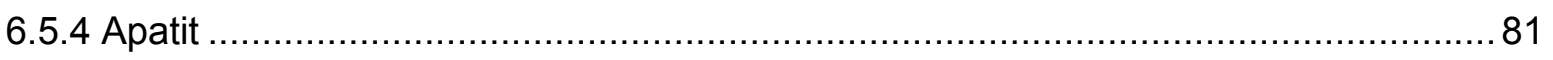

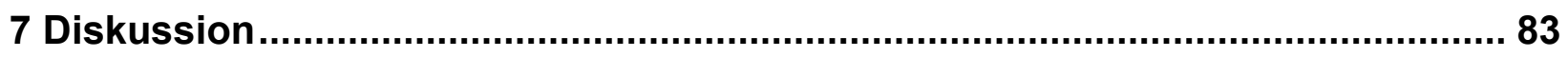

7.1 Beckengenese und Sedimentationsgeschichte des Eckfelder Maares ........................... 83

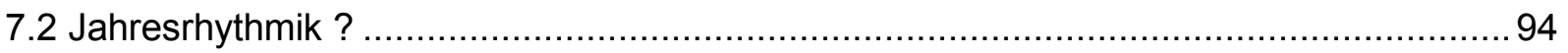

7.3 Die Profundalsedimente von Eckfeld im Vergleich mit den Seeablagerungen von Messel (Mitteleozän) und Enspel/Westerwald (Oberoligozän) ............................... 97

8 Zusammenfassung der Ergebnisse .................................................................... 98

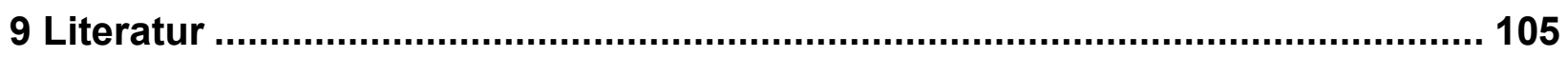

Dank

\section{Verzeichnis der Anhänge}

Anhang 1-1 bis 1-6

Anhang 2-1 bis 2-6

Anhang 2-7 und 2-8

Anhang 2-9 bis 2-12

Anhang 2-13

Anhang 3-1

Anhang 3-2

Anhang 3-3

\section{Verzeichnis der Anlagen}

Anlage 1

Anlage 2
Detail-Profile aus der Bohrung E3/96

Fototafeln: Laminen-Typen

Fototafeln: Resedimente

Fototafeln: Sedimentbestandteile

EDX-Spektren

Häufigkeitsverteilung der organischen und mineralischen Partikel (Bohrungen E1/96 und E2/96), tabellarisch

Verteilung und Koloniegrößen von Botryococcus cf. braunii (Bohrung E1/96 und E2/96)

Verzeichnis der Dünn- und Stückschliffe (Bohrung E3/96)
Profile der Bohrungen E1/96, E2/96 und E3/96

Häufigkeitsverteilung der organischen und mineralischen Partikel (Bohrungen E1/96 und E2/96), graphisch 


\section{Abkürzungen}

DM Durchlicht-Mikroskopie

I XPol. Gekreuzte Polarisatoren

AM Auflicht-Mikroskopie

AFM Auflicht-Fluoreszenz-Mikroskopie

REM Rasterelektronen-Mikroskopie

BSE Rasterelektronen-Mikroskopie im Rückstreuelektronen-Modus

EDX Energiedispersive Röntgenspektroskopie

XRD Röntgendiffraktometrie

LFZ Lithofazieszone

MFZ Mikrofazieszone

LT Laminen-Typ

SOM Sedimentäres organisches Material

POM Partikuläres organisches Material [im Folgenden synonym mit "Kerogen", siehe auch TYSON (1995, S. 18)]

NHM/LfN Naturhistorisches Museum Mainz/Landessammlung für Naturkunde Rheinland-Pfalz 

Rhythmically laminated sequences provide a fascinating stimulation for the human psyche.

(GLENN \& KELTS 1991)

\section{Einleitung}

Aufgrund ihrer zumeist geringen Ausdehnung und der - aus geologischer Sicht - kurzzeitigen Existenz stellen Seen überschaubare Lebensräume dar, deren ökologische Charakteristika oft vollständiger dokumentierbar sind als diejenigen im marinen Milieu (CLAUSING 2001). Relativ zu marinen Systemen reagieren Seen sensitiver auf schwankende Umweltbedingungen (KELTS 1988, GLENN \& KELTS 1991). Seesedimente gehören daher zu den besten Indikatoren für eine Rekonstruktion des Paläoklimas in kontinentalen Bereichen (TALBOT \& ALLEN 1996). Die Art und der Mechanismus des Sedimenteintrags sind in lakustrinen Ablagerungsräumen jedoch nicht nur abhängig vom jeweils herrschenden Klima (Abb. 01). Sie werden ebenso durch die Beckenmorphologie sowie die Topographie und Geologie des Einzugsgebietes gesteuert (GLENN \& KELTS 1991, ZOLITSCHKA 1998).

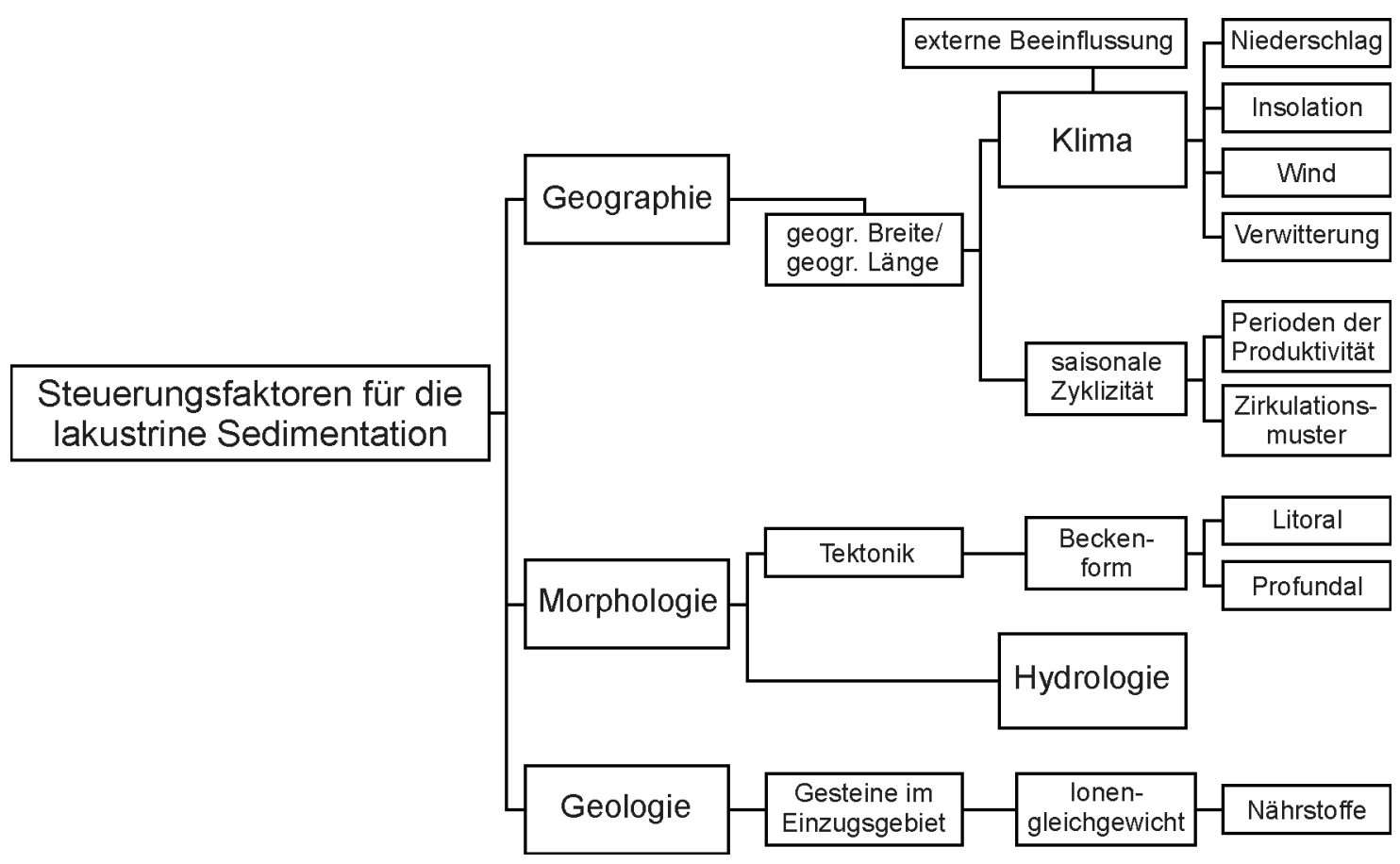

Abb. 01: Steuerungsfaktoren für die lakustrine Sedimentation (nach GLENN \& KELTS 1991).

Maarseen sind aufgrund ihres üblicherweise geringen Durchmessers, ihrer großen Tiefe und ihres begrenzten Einzugsgebietes ideale Forschungsobjekte für die ökologische Wechselwirkungen in limnischen Milieus. Angesichts des hohen Erhaltungspotentials und des Reichtums an überlieferten Bioindikatoren (LUTZ \& NEUFFER 2001) haben sich gerade die laminierten Seesedimente von Eckfeld in den letzten 20 Jahren als ein ausgezeichnetes Archiv zur Rekonstruktion paläoökologischer Steuerungsfaktoren während des mitteleozänen Klimaoptimums erwiesen. 


\section{Das Eckfelder Maar}

\subsection{Lage und geologischer Rahmen}

Das tertiäre Vulkanfeld der Hocheifel umfaßt rund 400 gegenwärtig bekannte Eruptionszentren. Nach Kalium-Argon-Datierungen begann die vulkanische Aktivität vor $45 \mathrm{Ma}$ im Mitteleozän und setzte sich mit wechselnder Intensität bis in das beginnende Miozän (vor 23-24 Ma) hinein fort (CANTAREL \& LIPPOLD 1977, MüLLER-SOHNIUS et al. 1989, MEYER 1994). Auf einer N-S bis NNE-SSW ausgerichteten Fläche von etwa $1000 \mathrm{~km}^{2}$ wurden überwiegend Glieder einer Alkalibasalt-Assoziation (Olivinbasalte, Alkaliolivinbasalte, Nephelinbasanite und Olivinnephelinite) gefördert. Höher differenzierte Hawaiite, Mugearite, Benmoreite und Trachyte sind in ihrem Auftreten auf das Kerngebiet des Vulkanfeldes bei Kelberg begrenzt (HUCKENHOLZ 1983, HUCKENHOLZ \& BÜCHEL 1988). Das Kelberger Hoch bezeichnet eine konzentrische, positiv magnetische Anomalie mit einem Durchmesser von etwa $20 \mathrm{~km}$ (Abb. 04). Als Ursache für das Störfeld im Zentrum des Vulkangebietes vermutet BüCHEL (1992) eine tertiäre Magmakammer in etwa $10 \mathrm{~km}$ Tiefe.
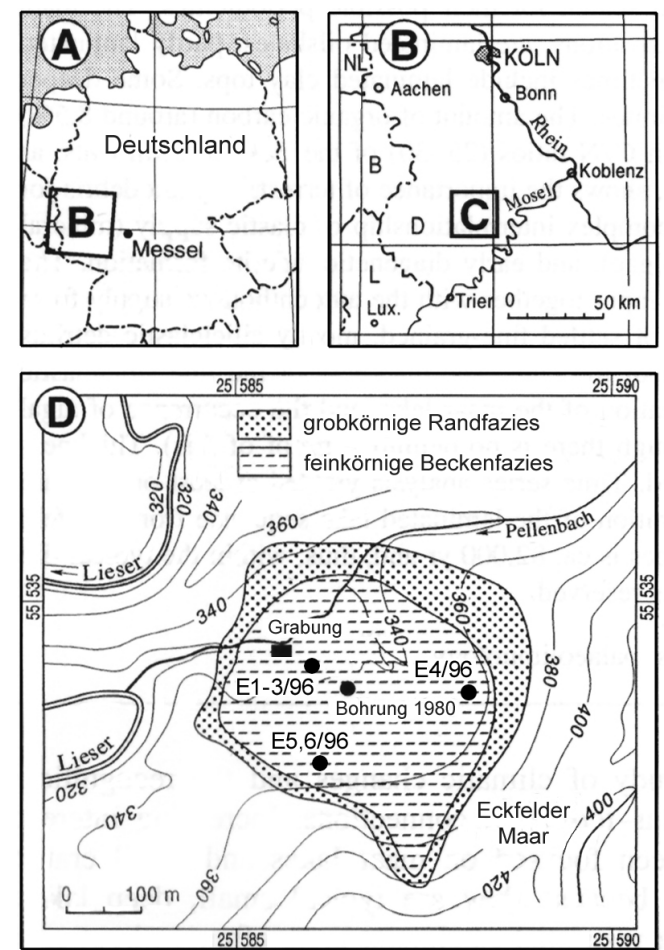

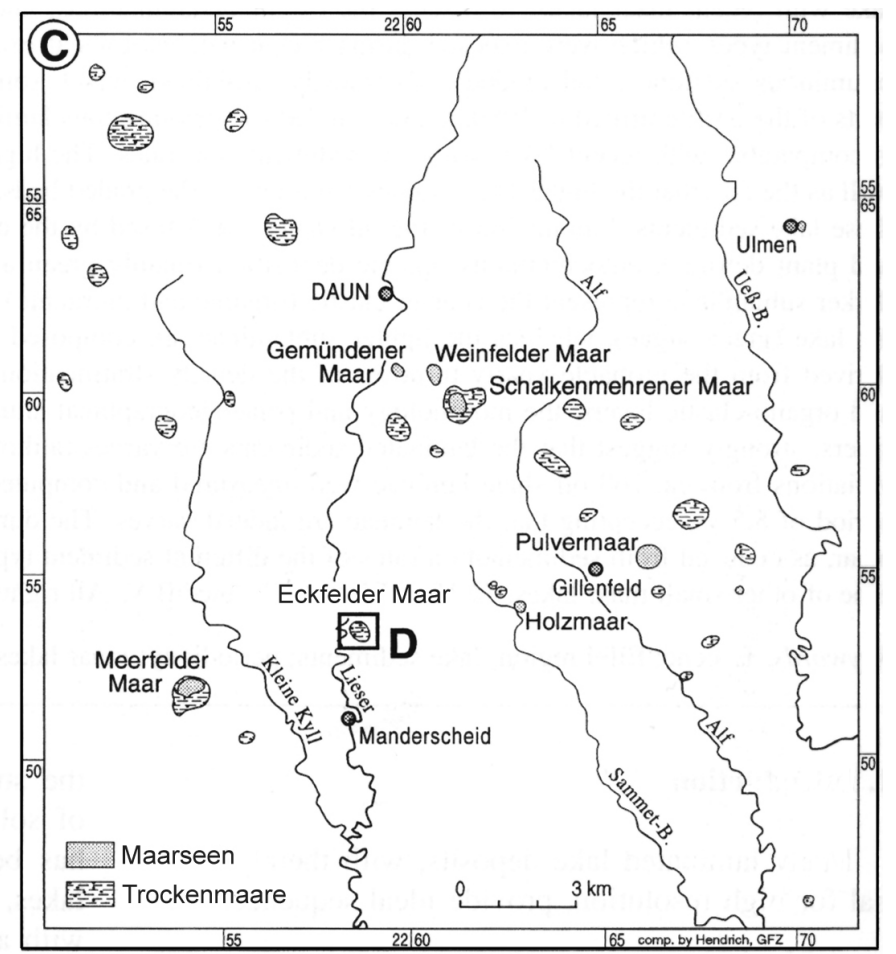

Abb. 02: (A bis C) Geographische Lage des mitteleozänen Eckfelder Maares, (D) Lokationen der Bohrungen sowie der Grabungsfläche (nach MINGRAM 1998 und PIRRUNG 1998).

Das mitteleozäne Eckfelder Maar liegt rund 20km SSW des Kelberger Hochs im Randbereich des tertiären Hocheifel Vulkanfeldes, etwa 1,5km nordwestlich der Gemeinde Eckfeld bei Manderscheid/Südwesteifel (BI. Daun, TK 25 Nr. 5806). Nahe der Einmündung des Pellenbaches in die Lieser, einem Nebenfluß der Mosel, streicht das Tertiärvorkommen isoliert in einer kesselförmigen, rund 450 x 500m messenden Erweiterung des Pellenbachtals aus (Abb. 02). Von lithologisch eintönigen Gesteinen der Unterems-Stufe (Unterdevon) umgeben punktiert das Maardiatrem die Nordwestflanke des Manderscheider Antiklinoriums, einer bedeutenden 
und kompliziert gebauten Großfaltenstruktur innerhalb der Südwesteifel (MEYER et al. 1994). Etwa 100m SE des Kraterrandes treten Siegen-Schichten zutage, die auch den Kern des Antiklinoriums aufbauen. Im Raum Manderscheid/Eckfeld wird das Unterdevon vorwiegend durch graue bis rotgraue oder auch grünlich graue Silt- und Sandsteine und quarzitische Sandsteine repräsentiert. Das Korngrößenspektrum liegt in der Regel zwischen Mittelsilt und Feinsand. Hinzu treten, wenn auch meist untergeordnet, Mittelsandsteine und Tonsteine bzw. Tonschiefer (KöLSCHBACH et al. 1993, MEYER et al. 1994).

Nach ZsÓTÉR (1997) lassen sich die am Südrand des Eckfelder Maares und im sogenannten "Ostschurf" (Schurf N5 von PIRRUNG 1992b) anstehenden Tuffe von einem basaltischen oder etwas höher differenzierten Magma ableiten. Demgegenüber lieferten geochemische Analysen von DEN BROK \& HOFMEISTER (2000) keinerlei Hinweise auf fraktionierte Förderprodukte.

\subsection{Klima und eozänes Relief im Raum Manderscheid/Eckfeld}

Neben den zahlreichen paläobotanischen Befunden läßt vor allem die in Eckfeld überlieferte Insekten-Taphozönose auf ein humides, tropisch-subtropisches Klima zur Bildungszeit der Seesedimente schließen (NICKEL 1994, 1996; LUTZ \& NEUFFER 1997, LUTZ et al. 2000, RUST 2002). Die Jahresdurchschnittstemperatur lag vermutlich deutlich über $20^{\circ} \mathrm{C}$ (LUTZ 1991, LUTZ et al. 1998). Nach MAI (1995) und Vos \& MINGRAM (2002) ist für das Mitteleozän Zentraleuropas von einem Monsun-Klima mit relativ trockenen Wintern und feuchten Sommern auszugehen.

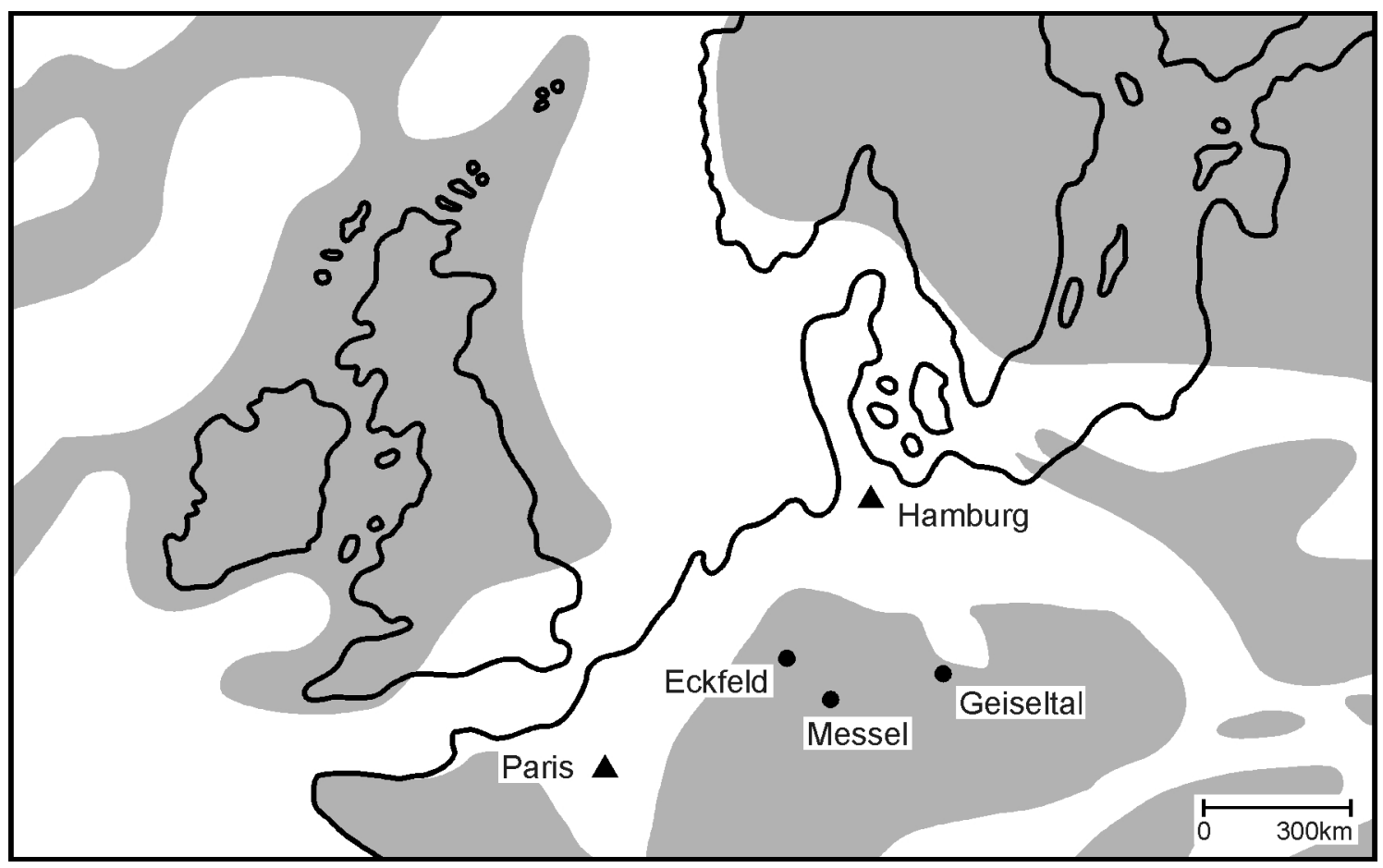

Abb. 03: Paläogeographie West-Europas im Mitteleozän (nach FRANZEN 1995 und WILDE \& FRANKENHÄUSER 1998).

Im Raum Manderscheid/Eckfeld war das eozäne Relief mit hoher Wahrscheinlichkeit durch eine bereits prätertiär angelegte, rund $200 \mathrm{~m}$ hohe Schichtstufe des Buntsandsteins geprägt (LUTZ et al. 2000). Sie kann auch als eine wirksame Wasserscheide zwischen dem Rheinischen 
Schiefergebirge und dem Pariser Becken angenommen werden (LÖHNERTZ 1994). Eine von Süden kommende und an die Buntsandsteinverbreitung angelehnte Ur-Saar-Talung schwenkte nördlich von Arenrath - evtl. durch das vom Kehlberger Hoch verursachte gegenläufige Gefälle - in eine SW-NE-Richtung analog dem heutigen Moselverlauf ein (Abb. 04). Im Gebiet des Arenrather Beckens mündeten kleinere aber vermutlich gefällreiche Zuflüsse in die Ur-Saar, in deren Einzugsbereich (Manderscheider Talung) auch das eozäne Vorkommen von Eckfeld einzuordnen ist (LÖHNERTZ 1994, LUTZ et al. 2000).

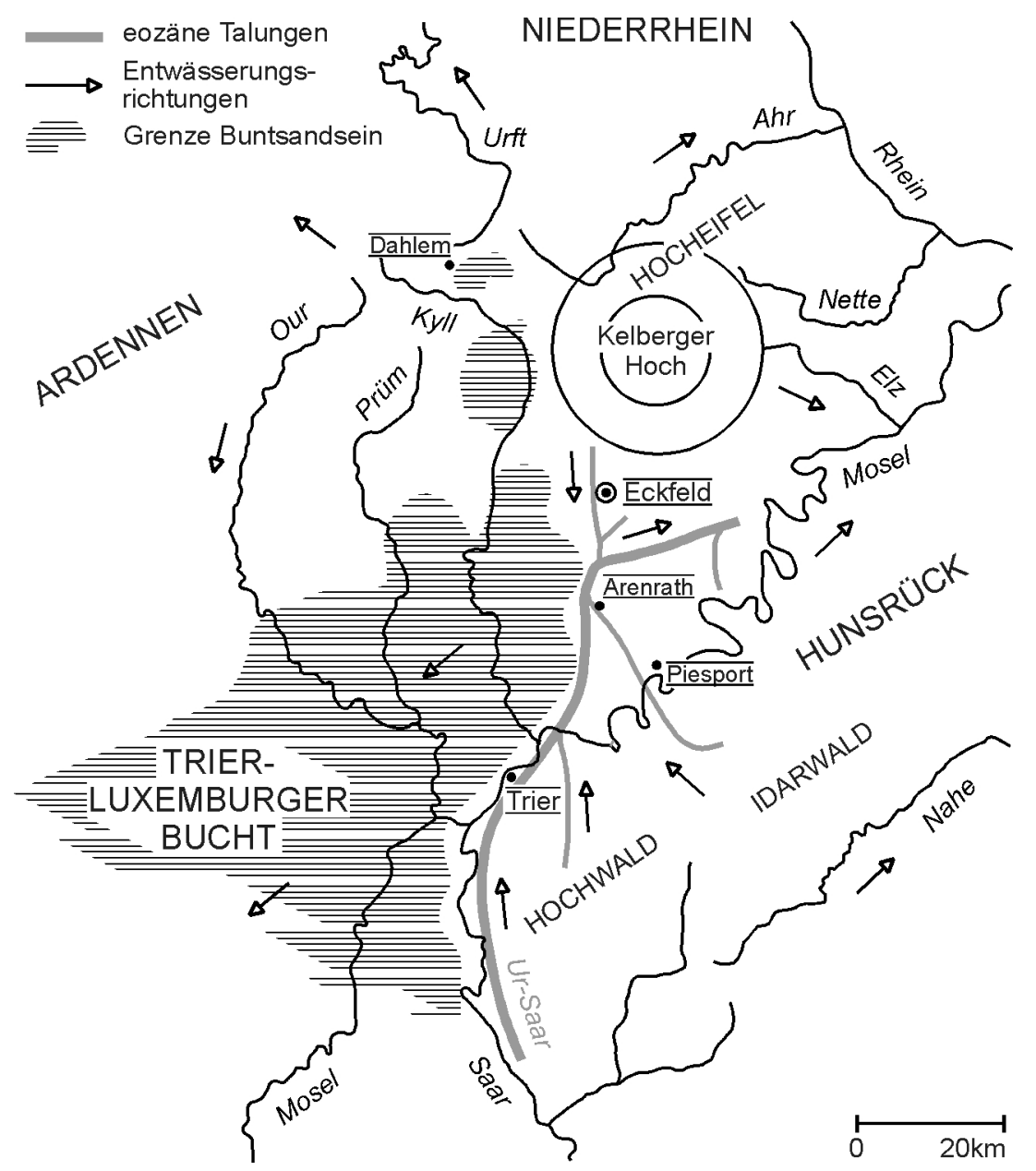

Abb. 04: Rekonstruktion des mitteleozänen Talsystems der Westeifel (nach LÖHNERTZ 1994).

\subsection{Erforschungsgeschichte und gegenwärtiger Kenntnisstand}

\section{Die Entdeckung des Tertiärvorkommens und erste Erkenntnisse}

Entdeckt wurde das Eckfelder "Braunkohlenlager" im Jahre 1838 von dem Dorflehrer Nikolaus Pauly aus Eckfeld, der mittels zweier Schächte auch versuchsweise den Abbau der "Braunkohle" betrieb (WEBER 1853, LÖHNERTZ 1978a).

Die erste wissenschaftliche Bearbeitung von WEBER (1853) beruhte auf Unterlagen des damaligen Königlichen Oberbergamtes Bonn. Aufgrund von Blattfunden ordnete er die Eckfelder "Braunkohlenablagerungen" dem Tertiär zu und stellte sie irrtümlich in einen stratigraphischen Bezug zu der "Niederrheinischen Braunkohlenformation". Nach seiner Auffassung entstand das Eckfelder "Kesselthal" durch eine isolierte, kleinräumige Auswaschung des 
"Grauwackengebirges" in der später Torfbildung stattfand. Im Gegensatz dazu vermutete v. DECHEN (1884, S. 587), daß "... in dem Raume zwischen Obermanderscheid, Eckfeld, Schutz und Daun nur die letzten Reste pflanzenführender oligocäner Ablagerungen vorhanden sind, welche ursprünglich viel grössere Flächen bedeckt und möglicher Weise sogar in Zusammenhang mit einander gestanden haben ...". Ähnlicher Ansicht war auch LEPSIUS (1887-1892). Er sah den scharfen Kontakt zwischen der "Braunkohlen-Ablagerung" und dem angrenzendem Devon als Indiz für eine "Grabenversenkung", die das Eckfelder Vorkommen vor der Denudation schützte.

Die erste palynostratigraphische Einstufung des "Kohlenvorkommens bei Eckfeld" nahm PFLUG (1959) vor. In der Mikroflorengesellschaft einer nicht näher lokalisierten Einzelprobe aus der Sammlung des Geologischen Institutes Köln erkannte er das "Borkener Bild" und ordnete sie nach damaligem Kenntnisstand dem Obereozän zu. Spätere pollenanalytische Untersuchungen an fünf relativ oberflächennah gewonnenen Bohrproben (maximale Teufe: $5 \mathrm{~m}$ ) durch V. D. BRELIE ergaben ebenfalls "Borkener Bild", welches nun aber durch eine zwischenzeitliche stratigraphische Revision ins Mitteleozän gestellt wurde (V. D. BRELIE et al. 1969). Hinsichtlich der Genese des Eckfelder Tertiärs griffen die Autoren WEBERS "Kesseltal-Theorie" erneut auf. Ihrer Meinung nach wurde das Eckfelder Tertiär "in einer Senke der präoligozänen Landoberfläche" abgelagert (ebd., S. 38). Während des Pleistozäns kam es dann zu einem "Kesseleinbruch" über einem erkalteten Magmaherd, der das Vorkommen in sein heutiges tiefes Niveau an der Sohle des Pellenbachtals brachte.

\section{Die Bohrung von 1980}

Bereits LÖHNERTZ (1978b) äußerte die Vermutung, daß die Eckfelder Schwarzpelite eine mitteleozäne Maarfüllung repräsentieren könnten. Erste Belege hierfür erbrachte eine von der Deutschen Forschungsgemeinschaft (DFG) finanzierte Bohrkampagne im Spätherbst 1980. Die Bohrung wurde nahe dem Zentrum des Tonvorkommens angesetzt und bis in eine Tiefe von 66,5m niedergebracht (LÖHNERTZ 1982, NEGENDANK et al. 1982).

Kernbeschreibung: An der Basis des Bohrprofils wurde eine rund $16 \mathrm{~m}$ mächtige Folge pyroklastischer Ablagerungen angetroffen, die sich in ihrem untersten Teil aus meist ungeschichteten Lapilli-Brekzien bis z.T. pyroklastischen Brekzien zusammensetzt (NEGENDANK et al. 1982). Die Lapilli bestehen überwiegend aus devonischem Nebengestein, aber auch aus verwitterten Vulkaniten. Im mittleren und oberen Abschnitt der Folge treten zunehmend geschichtete, teils gradiert geschichtete Lapilli-/Aschen-Tuffe auf. Ab 50,5m Teufe folgt eine rund 35m mächtige Sequenz aus sideritführenden, "bituminösen Laminiten" mit Einschaltungen von Diatomeenlagen. Nach einer 6,5m mächtigen "Übergangszone" aus "bituminösen Silten" folgen teils horizontalgeschichtete Tone und Silte, die NEGENDANK et al. (1982, S. 160) als "rein mineralische Sedimente" bezeichnen. Der oberste meist ungeschichtete Abschnitt dieser Ton/Silt-Folge ist als Hangschuttprofil mit Grauwacken-Klasten ausgebildet.

ZOLITSCHKA (1993) unterteilt das Bohrprofil in Anlehnung an die lithologische Kernbeschreibung von NEGENDANK et al. (1982) in 4 Lithozonen (Tab. 01). 
Tab. 01: Lithozonen der Bohrung von 1980

\begin{tabular}{|c|c|c|}
\hline Lithozone & Teufe $(\mathrm{m})$ & Lithologie [nach ZOLITSCHKA (1993)] \\
\hline D & $0,0-9,5$ & Ton und Silt Lamination \\
\hline C & $9,5-16,0$ & Übergangszone mit bituminösen Silten \\
\hline B & $16,0-50,5$ & laminierte lakustrine Ölschiefer mit Diatomeen und bituminösem Material \\
\hline A & $50,5-66,5$ & $\begin{array}{l}\text { horizontal geschichtete, umgelagerte Pyroklastika mit blasigen Basalt- } \\
\text { Lapilli und zertrümmertem devonischen Gestein }\end{array}$ \\
\hline
\end{tabular}

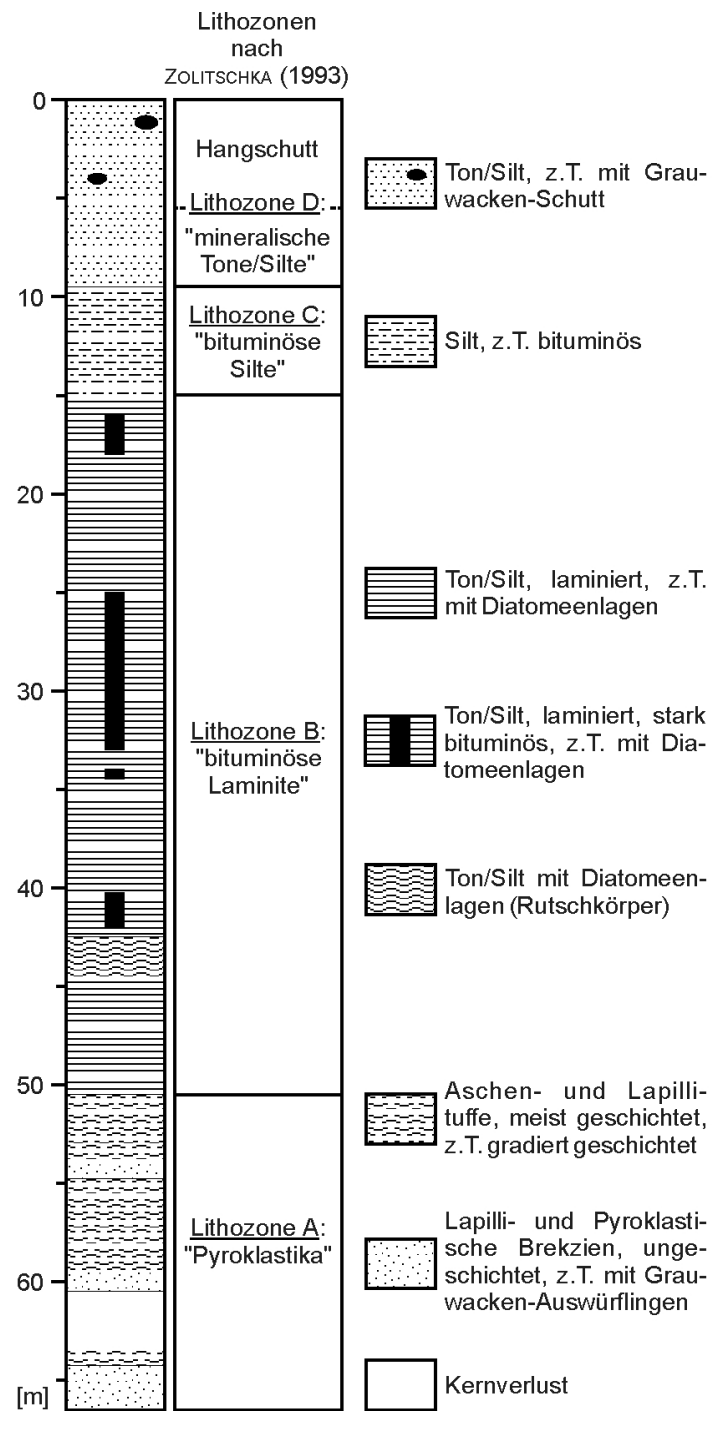

Abb. 05: Lithozonierung der Bohrung von 1980 (nach NEGENDANK et al. 1982 und ZOLITSCHKA 1993).

Interpretation: Der untere Bereich der "Pyroklastika-Folge" (Lithozone A) - die bei einer Endteufe von $66,5 \mathrm{~m}$ noch nicht durchbohrt wurde - ist nach NEGENDANK et al. (1982) als eine subaerisch abgelagerte Schlotbrekzie anzusprechen. Im oberen Abschnitt der Folge spiegeln umgelagerte "Tuffe" sowie das Auftreten der ersten Diatomeenlage bei $52 \mathrm{~m}$ die subaquatische Sedimentation in einem Maarsee wider. In den "bituminösen Laminiten" (Lithozone B) sehen NEGENDANK et al. (1982) die Hauptphase der See-Entwicklung dokumentiert. Das Auftreten von "bituminösen Silten" (Lithozone C) und "mineralischen Ablagerungen" (Lithozone D) im Hangenden werten sie "bei aller Vorsicht der Interpretation" (ebd., S. 159) als Hinweis auf eine beginnende Verlandungsphase des Sees.

Mit Hilfe zahlreicher Sondierungsbohrungen innerhalb des Maarkraters konnte PIRRUNG (1992a, 1993) organisch-reiche Laminite bis in eine Höhe von $395 \mathrm{~m}$ ü. NN nachweisen, also $52 \mathrm{~m}$ über dem Ansatzpunkt der Bohrung von 1980. Die in der Bohrung mit rund 50m Mächtigkeit überlieferten Laminite stellen also nur den unteren Teil einer ursprünglich mehr als $100 \mathrm{~m}$ mächtigen feinkörnigen Beckenfüllung dar. Die als "rein mineralische Sedimente" angesprochenen Pelite der Lithozone D sind innerhalb des Kesseltals in Höhenlagen zwischen $340 \mathrm{~m}$ und $380 \mathrm{~m}$ NN verbreitet. Es kann sich somit allein aufgrund ihrer vertikalen Verbreitung nicht um in situ lagernde Sedimente einer Verlandungsphase handeln, sondern vielmehr um verwitterte und bei Rutschungen umgelagerte Sedimentpakete, die in ihrer ursprünglichen faziellen Ausbildung eher den organisch-reichen Sedimenten der Lithozone B entsprochen haben dürften (PIRRUNG 1992b). 


\section{Die Grabungskampagnen (1987 - andauernd)}

Seit 1987 führt das Naturhistorische Museum Mainz/Landessammlung für Naturkunde Rheinland-Pfalz regelmäßig geologisch-paläontologische Grabungen in den Seesedimenten des Eckfelder Maares durch (BRAUER \& LUTZ 1990). Im Laufe der alljährlichen Grabungskampagnen wurden bis heute mehr als 25.000 Fossilien geborgen (LUTZ et al. 1998). Zahlenmäßig am stärksten vertreten sind botanische Funde, wie Blätter, Samen, Früchte und Blüten (DANNAT 2000). Hierbei spielen die Reste dicotyler Angiospermen, wie Walnußgewächse (Juglandaceae), Teegewächse (Theaceae) und Ulmengewächse (Ulmaceae) eine übergeordnete Rolle. Aber auch Blatt- und Blütenreste von Palmen (Arecaceae) sind im Fossilbericht häufig vertreten. Unter den Invertebratenfunden dominieren, neben zahlreichen Schnecken (Gastropoda), Muscheln (Bivalvia) und Muschelkrebsen (Ostracoda), die Insekten und hier, mit fast $90 \%$ aller Insektenfunde, die Käfer (Coleoptera) (FRANKENHÄUSER et al. 1997, SACHSE \& LUTZ 2001). Beachtenswert ist auch der Nachweis der weltweit ältesten Honigbiene Eckfeldapis electrapoides n.sp. (LUTZ 1993c). Amphibien und Vögel sind bislang nur durch wenige SkelettFragmente vertreten. Sehr viel häufiger kommen neben den Überresten von Reptilien, wie z.B. von Schildkröten (Testudines) und Krokodilen (Crocodilia), die mit mindestens fünf Arten vertretenen Knochenfische (Osteichthyes) vor. Das wohl bekannteste Fossil dürfte jedoch das 1991 geborgene vollständige Skelett einer trächtigen Urpferdchenstute (Propalaeotherium voigti) sein. Dieser und eine Reihe weiterer Säugetierfunde erlauben auch die zweifelsfreie Einstufung des Eckfelder Tertiärvorkommens in das MP13, dem jüngsten Niveau des Geiseltaliums (Mitteleozän) der europäischen Landsäugetier-Stratigraphie (FRANZEN 1993, 1994). Palynologische Untersuchungen von NICKEL $(1994,1996)$ bestätigen dieses Alter. Damit ist Eckfeld jünger als die Seesedimente von Messel (MP11) bzw. die untere Mittelkohle des Geiseltals (MP12) und ungefähr gleich alt mit der oberen Mittelkohle des Geiseltals (vgl. Abb. 03).

Der Grabungstätigkeit des NHM/LfN folgten bis heute eine ganze Reihe wissenschaftlicher Publikationen zur Sedimentologie und Paläontologie der Eckfelder Seeablagerungen. Sie sind größtenteils im Mainzer Naturwissenschaftlichen Archiv erschienen. Jährliche Forschungsberichte über das laufende "Projekt Eckfelder Maar" werden in den Mitteilung der Rheinischen Naturforschenden Gesellschaft veröffentlicht.

\section{Die Bohrungen von 1996}

Im Rahmen eines interinstitutionellen Kooperationsprojektes des Geologischen Landesamtes Rheinland-Pfalz, des GeoForschungsZentrums Potsdam und des Naturhistorischen Museums Mainz/Landessammlung für Naturkunde Rheinland-Pfalz wurden im Sommer 1996 sechs Kernbohrungen an drei verschiedenen Lokationen innerhalb des Eckfelder Maares niedergebracht (FRANKENHÄUSER et al. 1997). Während die Bohrungen Eckfeld 4/96, 5/96 und 6/96 in randlichen Bereichen des Tonvorkommens ansetzten, durchteuften die Bohrungen Eckfeld 1/96, 2/96 und 3/96 die feinkörnigen Sedimente der zentralen Seefazies (Abb. 02). Insgesamt wurden bei der Bohrkampagne rund 290m Kernmaterial gewonnen. Mit 123,1m sind die Sedimente des Beckenzentrums in der Bohrung E1/96 in ihrer größten Mächtigkeit dokumentiert. ${ }^{40} \mathrm{Ar} /{ }^{39} \mathrm{Ar}-$ Datierungen an einem Alkalibasalt aus $64,7 \mathrm{~m}$ Bohrtiefe lieferten ein inverses Isochronen-Alter von 44,3 \pm 0,4 Ma (MERTZ et al. 2000) und bestätigen somit das biostratigraphisch ermittelte Alter der Fossilfundstätte von 44-45 Ma (FRANZEN 1993). 
Kernbeschreibung: Im Teufenbereich zwischen 123,1m und 71,2m wurden lockere Schuttmassen und durch kalzitische Zemente verfestigte Brekzien erbohrt. Makroskopisch sichtbare Schichtungsgefüge fehlen in diesem Abschnitt des Bohrprofils. Das Sediment ist allein durch eine Mengenvariation verschiedenartiger Lithoklasten gegliedert. An der Basis des Profils dominiert fragmentiertes Devon. Untergeordnet kommen entfestigte, stark vertonte Klasten vor, die nach FISCHER (1999) Reste einer mesozoisch-tertiären Verwitterungsdecke repräsentieren. Im Hangenden dieser lithologisch monotonen Brekzien treten mafische Lapilli in verschiedenen Häufigkeiten und Verwitterungsgraden als Gesteinskomponente hinzu. Gradierte Sedimentsequenzen schalten sich ab einer Teufe von $71,2 \mathrm{~m}$ in das Schuttprofil ein. Die Dezimeter bis Meter mächtigen Normalgradierungen von Kies zu Ton/Silt treten etwa alle 10 Profilmeter auf. Dazwischen lagern matrixarme und durch Kalzit zementierte Brekzien (PIRRUNG 1998, FISCHER 1999). Oberhalb von $41 \mathrm{~m}$ setzen helle, feinkörnige Laminite ein, die schließlich zu den organisch-reichen Schwarzpeliten im Hangenden überleiten.

Nach Variationen der volumenspezifischen Suszeptibilität und der Dichte des Kernmaterials unterteilt PIRRUNG (1998) das Bohrprofil E1/96 in 6 Lithozonen (Tab. 02).

Tab. 02: Lithozonen der Bohrung E1/96

\begin{tabular}{|c|c|c|}
\hline Lithozone & Teufe $(\mathrm{m})$ & Lithologie [ ${ }^{1}$ nach PIRRUNG (1998) / ${ }^{2}$ nach FISCHER (1999)] \\
\hline $\mathrm{D} 2$ & $5,0-33,5$ & bituminöse Laminite ${ }^{1}$ / Schwarzpelit ${ }^{2}$ \\
\hline D1 & $33,5-41,3$ & graue Laminite $^{1}$ / Laminit, minerogen ${ }^{2}$ \\
\hline $\mathrm{C} 2$ & $41,3-60,0$ & Turbidite $^{1}$ / Gradierungen und Schutt ${ }^{2}$ \\
\hline C1 & $60,0-87,0$ & $\begin{array}{l}\text { Turbidite mit Bimsen }{ }^{1} / \text { Gradierungen und Schutt bzw. lithologisch } \\
\text { variable Brekzien, Schutt }^{2}\end{array}$ \\
\hline B & $87,0-113,0$ & Brekzien mit Bimsen ${ }^{1}$ / lithologisch variable Brekzien, Schutt ${ }^{2}$ \\
\hline A & $113,0-123,0$ & Nebengesteinsbrekzien ${ }^{1} /$ lithologisch monotone Brekzien, Schutt ${ }^{2}$ \\
\hline
\end{tabular}

Interpretation: Die korngestützten Brekzien der Lithozonen A und B können nach PIRRUNG (1998) und FISCHER (1999) als syneruptiv abgelagertes Bergsturzmaterial bzw. als Trümmerstromablagerungen gedeutet werden. Demgegenüber spiegelt das erste Auftreten gradierter Schichten in Lithozone C1 den Beginn einer subaquatischen Sedimentation und die Anlage eines Maarsees wider. Allerdings bestehen nur etwa ein Zehntel der grobklastischen Ablagerungen zwischen $71,2 \mathrm{~m}$ und $41,3 \mathrm{~m}$ aus Gradierungen. Den Großteil der von FISCHER (1999) als "Gradierungen mit Schuttlagen" zusammengefaßten Lithozonen C1 und C2 von PIRRUNG (1998) nehmen ungradierte Brekzien ein. Die laminierten Sedimente der folgenden Lithozonen D1 und D2 kamen in einem permanenten Maarsee zum Absatz und sind Gegenstand dieser Arbeit. 


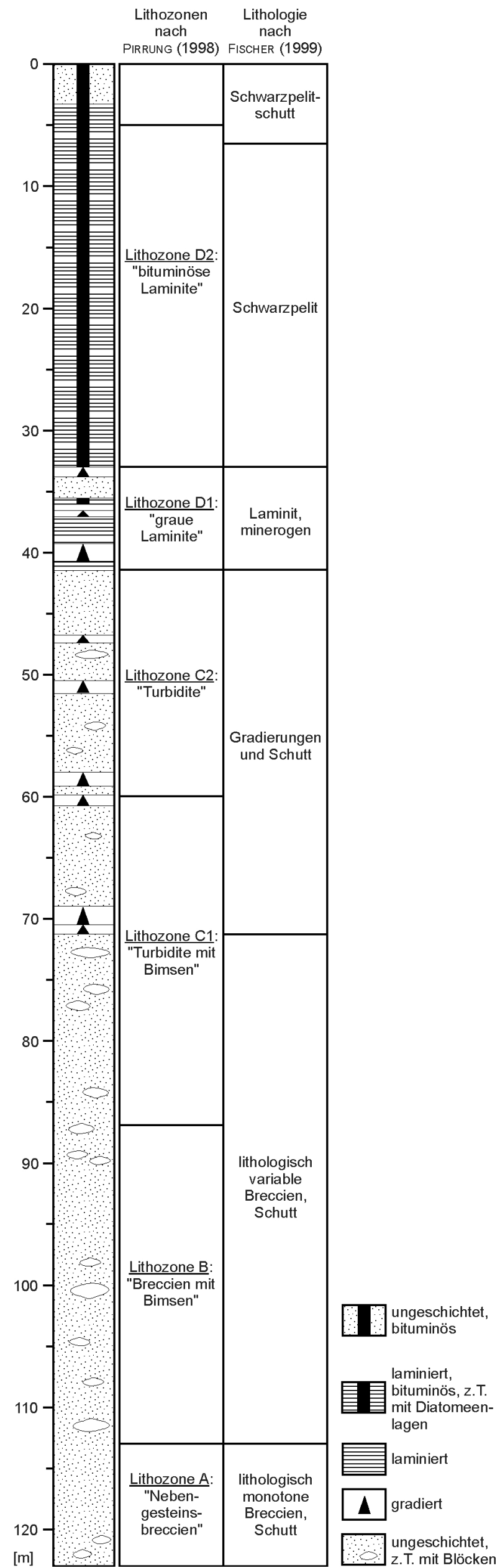

Abb. 06: Lithozonierung der Bohrung E1/96 (nach PIRRUNG 1998 und FISCHER 1999).

\section{Material und Methoden}

\subsection{Profilaufnahme und Zonierung}

Das untersuchte Kernmaterial entstammt den beckenzentralen Bohrungen Eckfeld 1/96, 2/96 und 3/96. Es liegt in insgesamt 71 Plexiglas-Inlinern mit einem Außendurchmesser $10,8 \mathrm{~cm}$ und einer Kernmarschlänge von ein bis zwei Metern (vgl. Anlage 1).

Als Grundlage für eine gezielte Probennahme wurden die Bohrungen E2/96 (Endteufe: 20,7m) und E3/96 (Endteufe: $39,2 \mathrm{~m}$ ) sowie die obersten 40,1m von E1/96 im Maßstab 1:20 aufgenommen und lithologisch beschrieben. Bereits makroskopisch im Profil erkennbare Variationen im Verteilungsmuster von organischer und mineralischer Substanz führten zu einer Unterteilung der Profundalsedimente in drei Lithofazieszonen (LFZ 1: Minerogene Laminite, LFZ 2: Übergangsschichten und LFZ 3: Biogene Laminite). Die Kerne sind untereinander laminengenau korrelierbar.

Bei der vollständigen Profilaufnahme wurde deutlich, daß sich die Halbkernstücke der Bohrung E3/96 am besten für eine Gewinnung ungestörten Probenmaterials eignen. Neun ausgewählte Profilabschnitte mit einer Länge zwischen $25 \mathrm{~cm}$ und $50 \mathrm{~cm}$ wurden im Maßstab 1:1 beschrieben (vgl. Anhang 1 und Anlage 1) und nachfolgend mitsamt der Plexiglas-Halbschalen aus den jeweiligen Kernmärschen herausgetrennt. Um die gering verfestigten Pelite dauerhaft vor Austrocknung und Oxidation zu schützen, aber auch um die weitere Herstellung von polierten Dünn- und Stückschliffen zu erleichtern, wurden die ausgesuchten Halbkernstücke nach der Methode von BULLWINKEL \& SosNITZA (2000) mit einem Zwei-KomponentenEpoxidharz imprägniert. 


\subsection{Probennahme und Präparation}

\section{Polierte Dünn- und Stückschliffe}

Die Herstellung hochpolierter Dünn- und Stückschliffe aus den imprägnierten Kernabschnitten der Bohrung E3/96 (vgl. Kap. 3.1) erfolgte nach dem von BULLWINKEL \& SOSNITZA (2000) entwickelten Verfahren. Es basiert auf der Tatsache, daß für die Schliffherstellung lediglich eine Oberflächenimprägnierung des Gesteins notwendig ist (ALLMAN \& LAWRENCE 1972). Auf eine vorherige physikalische oder chemische Trocknung des Sediments wird hierbei verzichtet. Auf diesem Wege wurden insgesamt 74 Gesteinsschliffe angefertigt (Anhang 3-3).

\section{Polierte Kornschliffe}

Die für eine detaillierte Mazeralanalyse bestimmten Kornschliffproben wurden den Bohrungen E1/96 und E2/96 entnommen. Beprobt wurden ausschließlich ungestört laminierte Profilbereiche, die keinerlei Anzeichen einer postsedimentären Umlagerung aufwiesen. Für jede Probe wurden zwischen $1 \mathrm{~cm}$ und $3 \mathrm{~cm}$ Sedimentsäule zusammengefaßt. Das gewonnene Material wurde auf Grobsandkorngröße zerkleinert und innerhalb von Ringformen (Durchmesser: $3 \mathrm{~cm}$ ) mit Epoxidharz imprägniert. Hierfür erwies sich das Injektionsharz EP der Firma RECKLI (Herne) als geeignet. Die entstandenen Sedimenttabletten wurden einseitig mit trockenem Korund-Schleifpapier in den Körnungen P400, P600, P800 und P1200 geschliffen. Eine abgestufte Politur der Proben erfolgte maschinell mittels einer $7 \mu \mathrm{m}-, 3 \mu \mathrm{m}-, 1 \mu \mathrm{m}$ - und $0,25 \mu \mathrm{m}$ Diamantpaste/Läppöl-Suspension. Die Kornschliffproben sind im Folgenden mit dem Kürzel "KS" versehen.

\subsection{Analyse und Auswertung}

\section{Auflicht- und Auflicht-Fluoreszenz-Mikroskopie}

(Mikroskop: LEITZ Ortholux mit Blaulicht-Fluoreszenzanregung; ÖI-Immersions-Objektiv, 50x)

Zur volumetrischen Bestimmung der partikulären organischen Substanz (POM) sowie ausgewählter mineralischer Komponenten (Diatomeen-Frusteln und Pyrit) wurde eine detaillierte Point-Count-Analyse (GLAGOLEV 1934) an 54 Körnerschliffen aus den Bohrungen E1/96 und E2/96 durchgeführt. Mit Hilfe der erweiterten Mazeralanalyse wurden die vertikalen Trends in der Verteilung von organischer und mineralischer Substanz erfaßt. Im Gegensatz zu der klassischen Mazeralanalyse, die lediglich organische Partikel einbezieht, wurde hier ausnahmslos jeder Punkt unter dem Fadenkreuz registriert, sofern es sich um Sediment handelte. Das ebenfalls aus der Pollenanalytik bekannte Problem der geschlossenen Datensätze ("closed data sets", KOVACH \& BATTEN 1994, LENZ 2000) konnte auf diese Weise aufgehoben werden. Pro Schliff wurden zwischen 646 und 2317 Punkte gezählt. Davon entfallen 0,1 Vol.\% (Probe KS1) bis 50,8 Vol.\% (Probe KS9) auf partikuläre organische Substanz (Anhang 3-1 und Anlage 2). Die Bestimmung der organischen Sedimentkomponenten erfolgte nach ICCP (1971), HUTTON (1987) und TAYLOR et al. (1998). 


\section{Durchlicht- und Auflicht-Fluoreszenz-Mikroskopie}

(Mikroskop: LEITZ Aristoplan mit Blaulicht-Floureszenzanregung)

Ziel der Untersuchungen an polierten Dünn- und Stückschliffen war es, die Art und den Gehalt an mineralischer Substanz im Sediment zu erfassen und charakteristische Schichtungsmerkmale zu typisieren. Da die Verteilung von organischer Substanz in der Regel feinsten Sedimentstrukturen folgt, lieferte die Auflicht-Fluoreszenz zusätzliche Informationen über diskrete Schichtungsphänomene. Zur systematischen Aufnahme primärer und sekundärer Gefügemerkmale diente die von COLE \& PICARD (1975) entwickelte Klassifizierung von Sedimentstrukturen in feinkörnigen Ablagerungen. Diese hat den Vorteil, daß Schichtungstypen zunächst rein beschreibend und nach einem einheitlichem Schema erfaßt werden, ohne zu einer vorschnellen Deutung der Ablagerungsbedingungen zu verleiten (vgl. Kap. 4).

Die Analysen führten zu einer Abgrenzung von diskreten Laminen-Typen (LT), die sich untereinander durch die Art und die Verteilung von mineralischer und organischer Substanz sowie durch den Charakter der daraus resultierenden Mikroschichtungsgefüge unterscheiden.

\section{Rasterelektronen-Mikroskopie}

(Mikroskop: LEO 1450VP mit systemintegrierter EDX-Analytik)

Die Rasterelektronen-Mikroskopie im Rückstreuelektronen-Modus ("backscattered electron mode", KRINSLEY et al. 1998) bietet eine hervorragende Ergänzung zu lichtoptischen Untersuchungsmethoden. BSE-Bilder von polierten Oberflächen liefern Kontraste der atomaren Ordnungszahl (Materialkontrast). Aufgrund dessen sind geringste Variationen im Verteilungsmuster von organischer und mineralischer Substanz in Schnitten senkrecht zur Schichtung erkennbar und hochauflösende Mikrotextur-Untersuchungen möglich. Simultan aufgezeichnete EDX-Spektren erlauben zusätzlich eine Bestimmung der Elementzusammensetzung von mineralischen und organischen Sedimentkomponenten. Die Messergebnisse wurden mit der Software INCA von Oxford Instruments verarbeitet. Kombinierte BSE/EDX-Analysen wurden an insgesamt acht hochpolierten, unbedampften Dünnschliffen durchgeführt (Anhang 3-3).

Von fünf Proben wurden Sedimentoberflächen rasterelektronenmikroskopisch untersucht (Anhang 3-3). Die mit Leit-C (nach GÖCKE) auf Aluminium-Träger befestigten, etwa Cent großen Sedimentstücke wurden zuvor mit Kohlenstoff bedampft.

\section{Röntgendiffraktometrie}

(Röntgendiffraktometer: Philips PW 1800)

Aus den mineralischen Sedimenten an der Basis der untersuchten Profilabfolge wurde eine Probe (Bohrung E3/96, Teufe: $38,60 \mathrm{~m}$ bis $38,58 \mathrm{~m}$ ) röntgendiffraktometrisch untersucht. Das bis auf Erbsengröße zerkleinerte Probenmaterial wurde mit der Kugelmühle 15 Minuten lang bei $270 \mathrm{u} / \mathrm{min}$ gemahlen. Nach dem Mahlvorgang wurde die Fraktion > 100 $\mu \mathrm{m}$ abgesiebt und erneut gemahlen. Zur abschließenden Messung mit dem Röntgendiffraktometer wurde das in Tablettenform gepreßte Gesteinspulver (Fraktion < 100 $\mu \mathrm{m}$ ) verwendet. Die Bestimmung der Mineralzusammensetzung erfolgte durch die Software APD 1700 von Philips. 
Für die Mineralbestimmung an organisch-reichen Proben erwies sich die oben beschriebene BSE/EDX-Analytik als die praktikablere Methode, da hierbei auf einen vorherigen $\mathrm{H}_{2} \mathrm{O}_{2}$ Aufschluß zur Entfernung störender organischer Verbindung verzichtet werden kann.

\section{Agglomerative Cluster-Analyse}

(Fusionsregel: Ward Methode; Distanzmaß: Euklidische Distanz)

Um vertikale Schwankungen in der Verteilung von organischer und mineralischer Substanz objektiv ermitteln zu können, wurde das statistische Verfahren der Cluster-Analyse auf den Point-Count-Datensatz (siehe oben) angewendet. In einem ersten Schritt wurden die errechneten Prozentwerte in Häufigkeitsklassen nach dem "logarithmic abundance class scheme" (GAUCH 1982) überführt. Die zehn Klassen sind dabei so gewählt worden, daß die prozentuale Reichweite ab der zweiten Häufigkeitsklasse doppelt so groß ist, wie die der jeweils vorangegangenen Klasse (Tab. 03). Durch die Transformation von Prozentwerten in einen vereinfachten Datensatz aus Häufigkeitsklassen werden kleinere Fluktuationen bei massenhaft auftretenden Partikeln unterbetont. Lediglich die grundlegenden, aussagekräftigen Signale bleiben signifikant erhalten.

Tab. 03: Prozentuale Reichweite der Häufigkeitsklassen

\begin{tabular}{ccccccccccc}
\hline Klassen & 0 & 1 & 2 & 3 & 4 & 5 & 6 & 7 & 8 & 9 \\
\hline \multirow{2}{*}{ Reichweite } & $0 \%$ & $>0 \%$ & $>0,5 \%$ & $>1 \%$ & $>2 \%$ & $>4 \%$ & $>8 \%$ & $>16 \%$ & $>32 \%$ & $>64 \%$ \\
\hline
\end{tabular}

Bei der agglomerativen Cluster-Analyse stellt jede Probe zunächst ein eigenes Cluster dar. Die Distanzen zwischen diesen Clustern werden durch das gewählte Distanzmaß (hier Euklidische Distanz) festgelegt. Es wird weiterhin eine Fusionsregel benötigt, um die Distanzen zwischen bereits bestehenden und neu gebildeten Clustern zu bestimmen. Prinzipiell werden stets diejenigen Cluster fusioniert, die die jeweils geringste Distanz (d.h. die jeweils größte Ähnlichkeit) zueinander aufweisen. Es wurde auf die "minimum variance clustering-" oder WardMethode (WARD 1963) zurückgegriffen. Dieses Verfahren wird als sehr effizient für die Clusterbildung angesehen (PIELOU 1984) und lieferte auch in diesem Fall nachvollziehbare Ergebnisse (vgl. Kap. 4). Bei der Ward-Methode wird die Gesamtsumme des Quadrates der Abweichung eines jeden Objektes vom Mittel (Zentroid) eines bereits bestehenden Clusters gemessen. Nach dem Vergleich aller möglichen Objektpaare wird jenes zusammengeführt, bei dem der Anstieg der Summe der Abweichung nach der Fusionierung am geringsten ist. Die Ergebnisse der Cluster-Analyse wurden in einem Dendrogramm dargestellt (Abb. 08). Der Interpretation eines clusteranalytisch erzeugten Dendrogramms sind prinzipiell keine Grenzen gesetzt. Es muß jedoch überprüft werden, inwieweit mit Hilfe der Cluster relevante Aussagen getroffen werden können. Demzufolge ist die Anzahl der Cluster, die aus einem Dendrogramm ausgeschieden werden, d.h. der Wert der "linkage distance", von dem aus die Gruppierung beginnt, entscheidend (LENZ 2000). In diesem Fall wurde für die Clusterbildung ein Grenzwert von 12 festgelegt. Alle Proben mit einer "linkage distance" unterhalb dieses Wertes wurden zu einem separaten Cluster zusammengefaßt. Die fünf erzeugten Proben-Cluster bilden, unter der 
Berücksichtigung sedimentologischer Befunde aus den Dünn- und Stückschliffuntersuchungen, die Basis für eine Abgrenzung von insgesamt acht Mikrofazieszonen (vgl. Kap. 4).

An 17 ausgewählten Kornschliffproben (vgl. Kap. 6.4.1 und Anhang 3-2) wurden zusätzlich die Größenvarianzen von insgesamt 3280 Botryococcus-Kolonien bestimmt und clusteranalytisch verarbeitet. Hierbei wurde wiederum das Euklidische Distanzmaß und die WardMethode als Fusionsregel verwendet. Es separierten zwei Probengruppen, die im Dendrogramm so deutlich voneinander getrennt sind, daß sich die Festlegung eines Grenzwertes der Clusterbildung erübrigt.

\section{Vertikale Zonierung der zentralen Seesedimente}

\section{Lithofazieszonen}

In einem ersten Schritt wurde die Sedimentsäule der korrelierbaren Profile E1/96, E2/96 und E3/96 in drei Lithofazieszonen unterteilt. Diese übergeordnete Profilzonierung stützt sich vorwiegend auf sedimentologische und sedimentpetrographische Aspekte der laminierten Pelitabfolge sowie der gröberklastischen Resedimente. Zusätzlich ging das volumenspezifische Verhältnis von mineralischer zu partikulärer organischer Substanz (POM) in die Zonierung ein.

Tab. 04: Lithofazieszonierung der zentralen Seesedimente (Bohrungen E1/96, E2/96 und E3/96)

\begin{tabular}{clcc}
\hline Lithofazieszone (LFZ) & \multicolumn{1}{c}{ Mineralische Substanz } & $\begin{array}{c}\text { POM } \\
\text { (Vol.\%) }\end{array}$ & Resedimente \\
\hline $\begin{array}{c}\text { 1: Minerogene Laminite } \\
\text { (vgl. Kap. 4.1) }\end{array}$ & $\begin{array}{l}\text { überwiegend allochthon [Quarz, Extraklasten] } \\
\text { und authigen [Pyrit] }\end{array}$ & 0,1 & $\begin{array}{c}\text { Typ-I } \\
\text { (vgl. Kap. 5.1.1) }\end{array}$ \\
$\begin{array}{c}\text { 2: Übergangsschichten } \\
\text { (vgl. Kap. 4.2) }\end{array}$ & $\begin{array}{l}\text { überwiegend allochthon [Quarz, Extraklasten] } \\
\text { und authigen [Pyrit] }\end{array}$ & 6,8 & $\begin{array}{c}\text { Typ-II } \\
\text { (vgl. Kap. 5.1.2) }\end{array}$ \\
$\begin{array}{l}\text { 3: Biogene Laminite } \\
\text { (vgl. Kap. 4.3) }\end{array}$ & $\begin{array}{l}\text { untraklasten] } \\
\text { und authigen [Opal(CT), Pyrit, Siderit] }\end{array}$ & 15,6 & $\begin{array}{c}\text { Typ-III } \\
\text { (vgl. Kap. 5.1.3) }\end{array}$ \\
\hline
\end{tabular}

\begin{tabular}{|c|c|c|}
\hline Bohrung von 1980 & \multicolumn{2}{|c|}{ Bohrung Eckfeld 1/96 } \\
\hline $\begin{array}{l}\text { Lithozonen } \\
\text { (NEGENDANK et al. 1982, ZOLITSCHKA 1993) }\end{array}$ & $\begin{array}{l}\text { Lithozonen } \\
\text { (PIRRUNG 1998) }\end{array}$ & $\begin{array}{l}\text { Lithofazieszonen } \\
\text { (diese Arbeit) }\end{array}$ \\
\hline \multicolumn{3}{|l|}{ D: Seesedimente (Phase 2) } \\
\hline C: Übergangszone & verwitterte Laminite & \multirow[b]{2}{*}{ LFZ 3: Biogene Laminite } \\
\hline B: Seesedimente (Phase 1) & LZ D2: bituminöse Laminite & \\
\hline \multirow{3}{*}{ A: Pyroklastikafolge } & \multirow{2}{*}{ LZ D1: graue Laminite } & LFZ 2: Übergangsschichten \\
\hline & & LFZ 1: Minerogene Laminite \\
\hline & & \\
\hline
\end{tabular}

Abb. 07: Vereinfachte Korrelation der Lithofazieszonen mit den bestehenden Lithozonierungen von PIRRUNG (1998) und der Bohrung von 1980 (NEGENDANK et al. 1982 und ZOLITSCHKA 1993). 


\section{Mikrofazieszonen}

Die jeweiligen Lithofazieszonen zeigen ausgeprägte vertikale Schwankungen im Verhältnis von minerogenen gegenüber biogenen Gesteinskomponenten, aber auch in der Häufigkeit von organischem Detritus relativ zu Primärproduzenten. Eine detaillierte Mazeralanalyse an insgesamt 54 Proben der Profile E1/96 und E2/96 führte daher, auch unter Berücksichtigung ausgewählter mineralischer Gesteinskomponenten (Diatomeen-Frusteln und Pyrit), zu der zusätzlichen Unterscheidung von insgesamt acht Mikrofazieszonen. Diejenigen Proben, die sich in bezug auf die Abundanzen der untersuchten Partikel ähnlich sind, wurden clusteranalytisch zu Probengruppen zusammengefaßt. Bei einer Ähnlichkeit ("linkage distance") von 12 (vgl. Kap. 3.3) separieren fünf Probencluster (Abb.08).

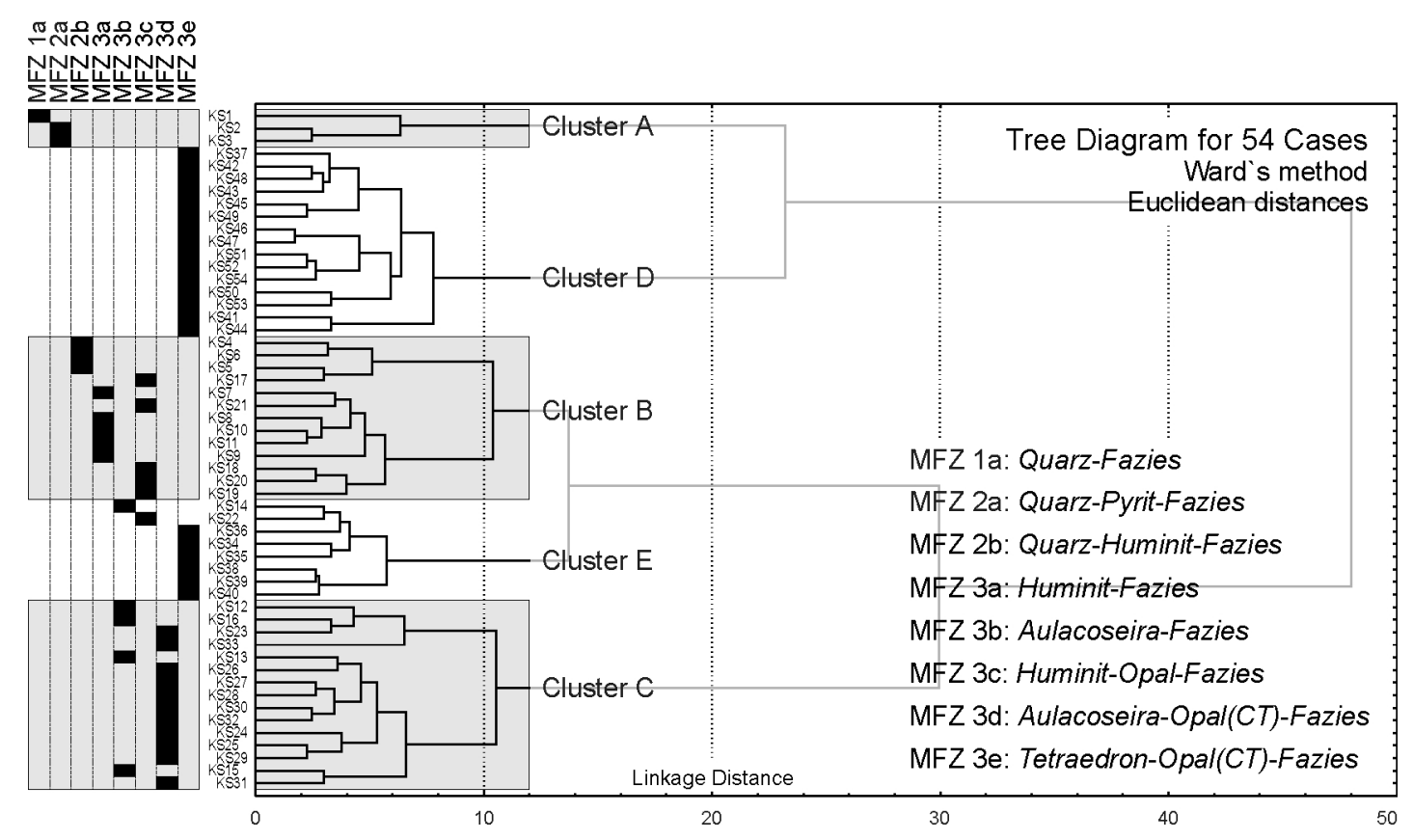

Abb. 08: Mikrofazieszonierung auf der Grundlage von fünf Probenclustern.

Tab. 05: Mikrofazieszonierung der zentralen Seesedimente (Bohrungen E1/96, E2/96)

\begin{tabular}{lll}
\hline \multicolumn{1}{c}{ Lithofazieszone (LFZ) } & \multicolumn{1}{c}{ Mikrofazieszone (MFZ) } & Cluster (vgl. Abb. 08) \\
\hline 1: Minerogene Laminite (vgl. Kap. 4.1) & 1a: Quarz-Fazies & A (1 von 3 Proben) \\
\hline 2: Übergangsschichten (vgl. Kap. 4.2) & 2a: Quarz-Pyrit-Fazies & A (2 von 3 Proben) \\
& 2b: Quarz-Huminit-Fazies & B (3 von 13 Proben) \\
\hline & 3a: Huminit-Fazies & B (5 von 13 Proben) \\
& 3b: Aulacoseira-Fazies & C (4 von 15 Proben); \\
& 3c: Huminit-Opal-Fazies & B (1 von 8 Proben 13 Proben); \\
3: Biogene Laminite (vgl. Kap. 4.3) & 3d: Aulacoseira-Opal(CT)-Fazies 8 Proben) & C (11 von 15 Proben) \\
& 3e: Tetraedron-Opal(CT)-Fazies & D (15 von 15 Proben); \\
& & E (6 von 8 Proben) \\
\hline
\end{tabular}


Cluster A beinhaltet die Proben von MFZ 1a: Quarz-Fazies (KS1) und MFZ 2a: Quarz-PyritFazies (KS2 und KS3). Das Sediment führt die höchsten im Profil registrierten Gehalte an Pyrit (3 Vol.\% bis 85 Vol.\%) und zeichnet sich durch äußerst niedrige POM-Werte (maximal 3,5 Vol.\%) aus. Dabei zeigt die Probe KS1 die deutlich geringsten Pyritgehalte und führt mit 0,1 Vol.\% kaum organische Substanz. Sie ist den Proben KS2 bzw. KS3 daher vergleichsweise unähnlich.

In den Proben KS7 bis KS11 bzw. KS18 bis KS21 des Clusters B tritt die Chlorophycee Botryococcus als einziger Vertreter der Primärproduzenten auf. Diese Proben sind bestimmend für MFZ 3a: Huminit-Fazies bzw. MFZ 3c: Huminit-Opal-Fazies. Die im Cluster verbleibenden Proben (KS4 bis KS6 und KS17) führen keine oder mit 0,1 Vol.\% nur sehr wenige Kolonien der Grünalge. Durch die weitgehende Abwesenheit von Primärproduzenten zeigen diese Proben ein überdurchschnittlich hohes Huminit:Liptinit-Verhältnis zwischen knapp 7 und über 15. Daraus resultiert die relative Unähnlichkeit zum Rest des Probenclusters. Während die Probe KS17 aufgrund ihrer Profillage der MFZ 3c zugerechnet wird, befinden sich KS4 bis KS6 im stratigraphischen Niveau von MFZ 2b: Quarz-Huminit-Fazies.

Für die 15 Proben des Clusters $\mathbf{C}$ ist die Präsenz von Diatomeen-Schalen als bedeutende Gesteinskomponente bestimmend. Mit Werten von > 1 Vol.\% kommen die Frusteln ausschließlich in MFZ 3b: Aulacoseira-Fazies (Probe KS12, KS13, KS15 und KS16) und MFZ 3d: Aulacoseira-Opal(CT)-Fazies (Probe KS23 bis KS33) vor. Die Proben KS12 und KS16 bzw. KS23 und KS33 markieren den jeweiligen Übergang zu den diatomeenfreien bzw. -armen Mikrofazieszonen 3a: Huminit-Fazies, 3c: Huminit-Opal-Fazies und 3e: Tetraedron-Opal(CT)Fazies. Sie sind den anderen Proben des Clusters entsprechend unähnlich.

Cluster D enthält 15 Proben (KS37 und KS41 bis KS54), die durch das häufige Auftreten der Chlorophycee Tetraedron gekennzeichnet sind. Das Probencluster ist für MFZ 3e: TetraedronOpal(CT)-Fazies bestimmend.

Cluster E umfaßt acht indifferente Proben, die zwar Diatomeen-Frusteln und/oder Tetraedron führen, durch deren geringe Konzentrationen aber nicht in die Probencluster $\mathrm{C}$ bzw. D fallen. Die Probe KS14 liegt im mittleren Abschnitt der MFZ 3b: Aulacoseira-Fazies, ist jedoch durch vergleichsweise geringe Gehalte an Diatomeen-Schalen charakterisiert. Mit nur 0,2 Vol.\% Diatomeen bildet die Probe KS22 den petrographischen Übergang von der ansonsten diatomeenfreien MFZ 3c: Huminit-Opal-Fazies zu der diatomeenreichen MFZ 3d: AulacoseiraOpal(CT)-Fazies. Die Proben KS34 bis KS36 bzw. KS38 bis KS40 führen jeweils geringe Werte für Diatomeen-Frusteln (maximal 0,8 Vol.\%) und Tetraedron (maximal 0,9 Vol.\%). Sie werden der MFZ 3e: Tetraedron-Opal(CT)-Fazies zugerechnet. Der stratigraphische Wechsel von der diatomeenreichen MFZ 3d zu der Tetraedron-reichen MFZ 3e ist jedoch graduell und markiert keinen primären Wandel in der Primärproduzenten-Vergesellschaftung. Er ist vielmehr ein Resultat der fortschreitenden Opal-Diagenese in diesem Profilabschnitt (vgl. Kap. 4.3.5).

Innerhalb einer jeden Mikrofazieszone wurden die gefügebildenden Elemente nach ihrem Stoffbestand, ihrer Textur sowie der Morphologie ihrer Grenzflächen charakterisiert und als Laminen-Typen (LT) ausgeschieden. 


\subsection{Lithofazieszone 1: Minerogene Laminite}

Teufe:

E1/96: $41,30 \mathrm{~m}$ bis $37,67 \mathrm{~m}$

E3/96: $39,20 \mathrm{~m}$ (Endteufe) bis $36,79 \mathrm{~m}$

Die Lithofazieszone 1: Minerogene Laminite an der Basis der untersuchten Kernabschnitte von E1/96 und E3/96 korrespondiert mit den unteren rund 3,6m der Lithozone "LZ D1: graue Laminite" von PIRRUNG (1998). Innerhalb der Minerogenen Laminite bildet die Quarz-Fazies die einzige Mikrofazieszone.

\subsubsection{Mikrofazieszone 1a: Quarz-Fazies}

$\begin{array}{ll}\text { Detail-Profil: } & \text { ML/Q-F1 (Anhang 1-1) } \\ \text { Dünn- und Stückschliffe: } & \text { ML/Q-F1.1 - 1.10 (Anhang 1-1) } \\ \text { Kornschliffe: } & \text { KS1 (Anlage 2) }\end{array}$

Tab. 06: Laminen-Typen der Mikrofazieszone 1a: Quarz-Fazies (vgl. Abb. 09)

\begin{tabular}{|c|c|c|}
\hline $\mathrm{Nr}$ & Laminen-Typ (LT) & Schichtgefüge \\
\hline $1 \mathrm{a}-1$ & Homogene quarzsandreiche Ton/Siltlagen & strukturlos \\
\hline $1 a-2$ & Quarzsandführende Siltlagen & eben parallel bis wellig parallel \\
\hline $1 a-3$ & Quarzsandführende Ton/Siltlagen & eben parallel bis wellig parallel \\
\hline $1 \mathrm{a}-4$ & $\begin{array}{l}\text { Anreicherungen von matrixgestützt eingelagerten } \\
\text { Quarzkörnern }\end{array}$ & $\begin{array}{l}\text { wellig parallel bis unterbrochen eben } \\
\text { parallel }\end{array}$ \\
\hline $1 a-5$ & $\begin{array}{l}\text { Lagen von komponentengestützt eingelagerten } \\
\text { Quarzkörnern, extraklastenführend }\end{array}$ & eben parallel bis wellig parallel \\
\hline
\end{tabular}

Das Sediment der Mikrofazieszone 1a besteht in weiten Teilen aus einer dunkelgrauen, chlorit- und hellglimmerreichen Tonmatrix mit isoliert und regellos eingelagerten Quarzkörnern (LT 1a-1). Die Quarze zeigen einen generell niedrigen Rundungsgrad (eckig bis kantengerundet) und treten in einem engen Korngrößenspektrum zwischen $15 \mu \mathrm{m}$ und $80 \mu \mathrm{m}$ auf (Anhang 2-1, Fig. 01). Quarzkorndurchmesser bis 120um sind nur in Ausnahmefällen innerhalb der strukturlosen Sedimentabschnitte nachzuweisen. Abgesehen von Quarz wird die Siltfraktion zu einem Großteil durch körnigen, meist $<7 \mu \mathrm{m}$ großen Anatas, einem Ti-Oxid der Rutil-Gruppe $\left(\mathrm{TiO}_{2}\right)$, repräsentiert. Die eckigen bis kantengerundeten Oxid-Körner treten isoliert und statistisch im Sediment verteilt auf oder bilden vereinzelt lockere Mineralcluster von rund $50 \mu m$ Durchmesser (Anhang 2-1, Fig. 02).

Den strukturlosen Peliten (LT 1a-1) sind in unregelmäßigen Abständen 0,1 - 1mm mächtige, weitgehend monomineralische Quarzkornanreicherungen (LT 1a-4) zwischengeschaltet. Nur die mächtigeren Schichten aus matrixgestützten Grobsilt- bis Feinsandkörnern halten über die gesamte Schliffbreite aus. Dünnere, oft nur einen oder zwei Korndurchmesser mächtige Horizonte sind dagegen häufig unterbrochen (Anhang 2-1, Fig. 04) oder keilen gelegentlich auch vollständig aus. Weiterhin treten $<1,5 \mathrm{~mm}$ mächtige Lagen von Quarzkörnern (LT 1a-5) auf, die im Unterschied zu den locker gepackten Quarzanreicherungen (LT 1a-4) durch ein überwiegend komponentengestütztes Gefüge und deutliche Anteile an siltkörnigen Ti-Oxiden ge-

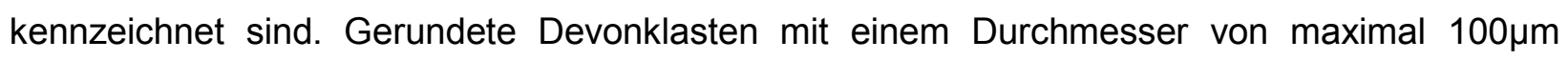


können am Schichtaufbau beteiligt sein. Die Kornlagen sind im allgemeinen ungradiert. Nur in Ausnahmefällen ist ein gradueller Übergang zu den hangenden Peliten durch eine Auflockerung des Korngefüges im obersten Abschnitt einer quarzreichen Schicht zu beobachten (Anhang 2-1, Fig. 03).

Unscharfe Alternationen von jeweils $0,2 \mathrm{~mm}$ bis $1,5 \mathrm{~mm}$ mächtigen silt- (LT 1a-2) und tonreichen Laminen (LT 1a-3) bilden einen weiteren Grundtyp der Sedimentation (Anhang 2-1, Fig. 05). Die insgesamt mehrere Millimeter bis Zentimeter mächtigen Hell/Dunkel-Wechselfolgen entsprechen den unstrukturierten Peliten im Mineralbestand. Während Ti-Oxide und siltgroße Quarze in den hellen Lagen (LT 1a-2) angereichert sind, aber auch in den dunklen Laminen (LT 1a-3) auftreten können, kommen statistisch verteilte Quarzsandkörner mit gleicher Häufigkeit in beiden Laminen-Typen vor. Die Schichtung wird jedoch vorwiegend durch eine Anreicherung von Tonpartikeln in den dunklen Laminen hervorgerufen (Anhang 2-1, Fig. 06). Weder die tonarmen noch die tonreichen Horizonte zeigen eine Gradierung der beteiligten Korngrößen.

Pyrit ist in framboidaler Form mit rund 3 Vol.\% (Probe KS1) am Sedimentaufbau beteiligt. Innerhalb der strukturlosen Profilabschnitte (LT 1a-1) sind isolierte Pyritframboide oder etwa $60 \mu \mathrm{m}$ große Framboid-Aggregate dispers in der feinkörnigen Matrix verteilt. Demgegenüber zeigen die geschichteten Profilbereiche oftmals eine Konzentration von Pyritframboiden in den tonreichen Horizonten (LT 1a-3).

Die minerogenen Ablagerungen der MFZ 1a führen nur sehr geringe Gehalte an partikulärer organischer Substanz. Organische Partikel, wie Ulminit, Sporinit und Liptodetrinit, kommen jeweils nur als isolierte Einzelexemplare vor. Bezogen auf das Gesamtvolumen des Sediments unterschreitet ihre jeweilige Häufigkeit jedoch die Möglichkeit einer sinnvollen Prozentwertdarstellung. Insgesamt haben organische Partikel mit lediglich 0,1 Vol.\% Anteil an der Sedimentzusammensetzung.

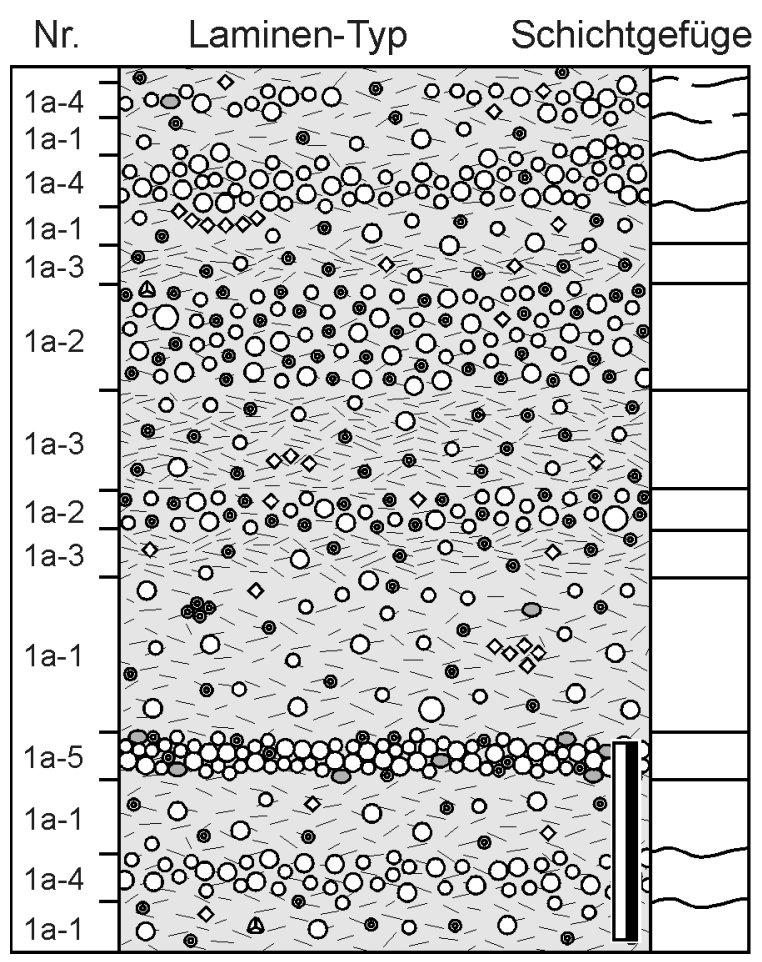

Komponenten

$\begin{array}{ll}0 & \text { Ton } \\ 0 \circ & \text { Quarz } \\ 0 & \text { Extraklasten } \\ 0 & \text { Ti-Oxide } \\ \diamond \diamond \diamond & \text { Pyrit }\end{array}$

Schichtgefüge

Abb. 09: Laminen-Typen der Mikrofazieszone 1a: Quarz-Fazies (Klassifizierung der Schichtgefüge nach COLE \& PICARD 1975). 


\subsection{Lithofazieszone 2: Übergangsschichten}

Teufe:

E1/96: $37,67 \mathrm{~m}$ bis $33,15 \mathrm{~m}$

E3/96: $36,79 m$ bis $32,65 m$

Die Sequenz der Übergangsschichten umfaßt den oberen Abschnitt der Lithozone "LZ D1: graue Laminite“ von PIRRUNG (1998). Innerhalb dieses Profilbereichs sind organische Partikel erstmalig mit Werten von $>1,0$ Vol. $\%$ am Sedimentaufbau beteiligt und bilden teilweise auch diskrete organisch-reiche Lagen. Der Anteil siliziklastischer Einschaltungen bleibt jedoch unverändert hoch. Da in den Übergangsschichten der grundlegende Wechsel von einer rein mineralischen Sedimentation im Liegenden (Lithofazieszone 1: Minerogene Laminite) hin zu den organisch-reichen Ablagerungen im Hangenden (Lithofazieszone 3: Biogene Laminite) überliefert ist, kommt innen eine entscheidende Bedeutung bei der Rekonstruktion der frühen SeeEntwicklung zu (BULLWINKEL \& RIEGEL 2000). Eine explizite Abgrenzung dieses Sedimentabschnitts innerhalb der "LZ D1: graue Laminite" von PIRRUNG (1998) ist somit notwendig.

Die Lithofazieszone 2: Übergangsschichten wird innerhalb der Eckfelder Profundalsedimente durch die Mikrofazieszone 2a: Quarz-Pyrit-Fazies und die nachfolgende MFZ 2b: QuarzHuminit-Fazies vertreten. Sie schließt in den zentrumsnahen Bohrungen E1/96 und E3/96 mit einem rund 2,6m mächtigen Konglomerat (vgl. Kap. 5.1.2) ab.

\subsubsection{Mikrofazieszone 2a: Quarz-Pyrit-Fazies}

Teufe:

E1/96: $37,67 \mathrm{~m}$ bis $37,17 \mathrm{~m}$

$\mathrm{E} 3 / 96: 36,79 \mathrm{~m}$ bis $36,35 \mathrm{~m}$

Detail-Profil:

ÜS/QP-F1 (Anhang 1-2)

Dünn- und Stückschliffe:

ÜS/QP-F1.1 - 1.5 (Anhang 1-2)

Kornschliffe:

KS2 u. KS3 (Anlage 2)

Tab. 07: Laminen-Typen der Mikrofazieszone 2a: Quarz-Pyrit-Fazies (vgl. Abb. 10)

\begin{tabular}{clc}
\hline Nr. & \multicolumn{1}{c}{ Laminen-Typ (LT) } & Schichtgefüge \\
\hline $2 a-1$ & Homogene quarzkornführende Pyritlagen & strukturlos \\
$2 a-2$ & $\begin{array}{l}\text { Pyritführende Anreicherungen von matrixgestützt eingelagerten } \\
\text { Quarzkörnern } \\
\text { 2a-3 }\end{array}$ & $\begin{array}{l}\text { Pyritführende Lagen von komponentengestützt eingelagerten } \\
\text { Quarzkörnern, extraklastenführend }\end{array}$ \\
\hline
\end{tabular}

Das kennzeichnende Merkmal der rund $50 \mathrm{~cm}$ mächtigen Mikrofazieszone 2a an der Basis der Übergangsschichten ist das massenhafte Auftreten von Pyrit. In den Proben KS2 und KS3 ist Pyrit mit über 85 Vol.\% bzw. rund 50 Vol.\% am Sedimentaufbau beteiligt und somit als gesteinsbildend $z u$ bezeichnen. Der im Vergleich zu den liegenden Minerogenen Laminiten stark erhöhte Pyritgehalt dieses Kernabschnitts bedingt einen bereits makroskopisch auffälligen Wechsel in der Sedimentfarbe von hellgrau nach dunkelgrau (Anhang 1-2).

Pyrit tritt häufig in Form dichter, strukturloser Massenansammlungen von rund $1 \mu \mathrm{m}$ großen xeno- bis hypidiomorphen Kristallen auf, die andere Gesteinskomponenten vollständig umschließen (Anhang 2-1, Fig. 07). Oftmals sind fließende Übergänge von formlosen Pyritkristall-Ansammlungen hin zu horizontal gelängten Pyrit-Framboid-Aggregaten (Polyframboide) 
zu beobachten (Anhang 2-1, Fig. 08). Aufgrund des hohen Pyritisierungsgrades des Sediments sind primäre Schichtungsgefüge nur sehr undeutlich erhalten geblieben und am ehesten durch die ungleichmäßige Verteilung von Quarzkörnern nachzuvollziehen. Ähnlich wie in der liegenden Lithofazieszone 1 lassen sich strukturlose Horizonte mit matrixgestützt eingelagerten Grobsilt- bis Feinsandquarzkörnern (LT 2a-1) von üblicherweise $<1 \mathrm{~mm}$ mächtigen, ungradierten Quarzanreicherungen mit matrix- (LT 2a-2) oder komponentengestütztem Korngefüge (LT 2a-3) unterscheiden. Letztere können feinsandgroße Devonklasten führen. Sie werden in einigen Fällen konkordant von leistenförmigen Pyrit-Framboid-Aggregaten unterlagert. Die oft mehrere $100 \mu \mathrm{m}$ langen und $20 \mu \mathrm{m}$ bis $50 \mu \mathrm{m}$ mächtigen Polyframboide im Liegenden der Kornlagen sind durch "load casts" unterhalb von Devonklasten gekennzeichnet. Sie bilden offenbar ehemalige Sedimentoberflächen nach, die zum Zeitpunkt des siliziklastischen Ablagerungsereignisses bereits durch synsedimentär gebildete, metastabile Fe-Sulfide oder Pyrit verfestigt waren und im Zuge der Sedimentkompaktion deformiert wurden (vgl. Kap. 6.5.1).

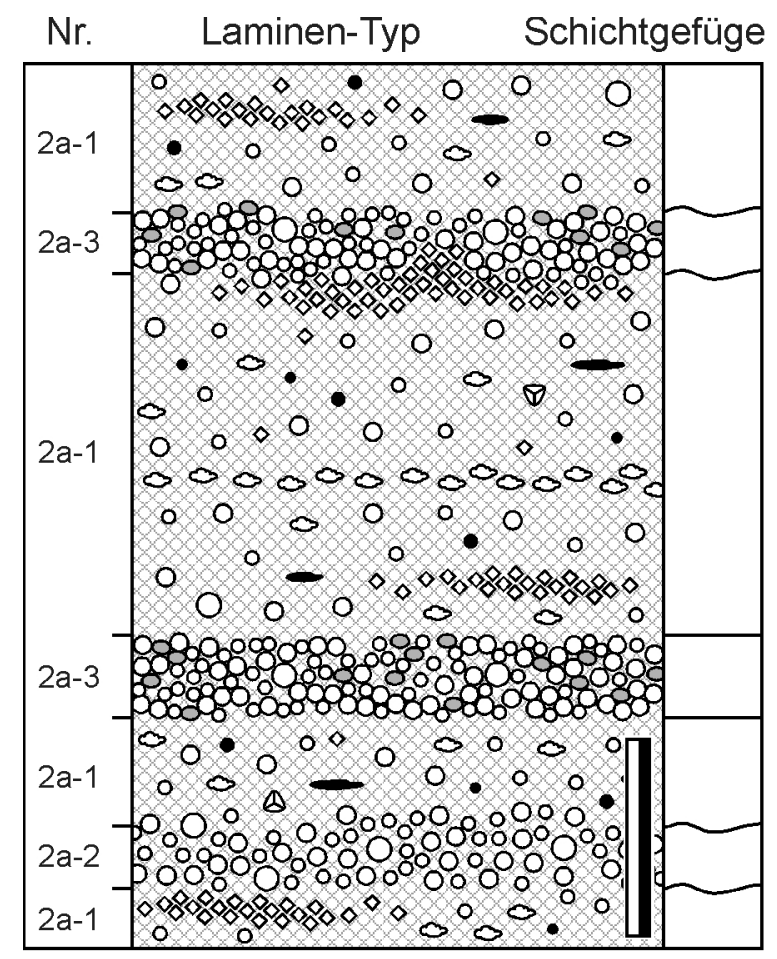
$\begin{array}{ll}\otimes \diamond \diamond & \text { Pyrit (strukturlos) } \\ \diamond \diamond \quad \text { Pyrit (framboidal) }\end{array}$
$0^{\circ}$ Quarz
${ }^{\circ}$ Extraklasten
-- Huminit
$\Delta$ Sporinit
o $\circ$ Botryococcus

Schichtgefüge

Abb. 10: Laminen-Typen der Mikrofazieszone 2a: Quarz-Pyrit-Fazies (Klassifizierung der Schichtgefüge nach COLE \& PICARD 1975).

Neben dem gehäuften Vorkommen von Pyrit ist die Mikrofazieszone 2a: Quarz-Pyrit-Fazies durch das erstmalige Auftreten partikulärer organischer Substanz in nennenswerter Quantität gekennzeichnet. An der Basis der Mikrofazieszone (Probe KS2) liegt der Gesamtgehalt an organischen Partikeln mit knapp 2 Vol.\% bereits um das Zehnfache höher als in den liegenden Minerogenen Laminiten, um dann in Probe KS3 auf 3,5 Vol.\% anzusteigen.

Unter den allochthonen Partikeln bilden Ulminit, Humodetrinit, Detro-Gelinit und isoliert auftretender Corpohuminit den Hauptanteil der organischen Sedimentkomponente. Der siltkorngroße huminitische Detritus (Humodetrinit, Detro-Gelinit und Corpohuminit) ist zusammen mit einzelnen Sporiniten regellos vorwiegend in den homogenen Quarz/Pyrit-Horizonten (LT 2a-1) 
verstreut. Auch das Vorkommen von maximal 250 $\mu$ m langen, pflanzlichen Gewebe-Fragmente (Ulminit) zeigt keine Bindung an diskrete Horizonte. Die unvollständig vergelte Form des Ulminits (Texto-Ulminit) ist in diesem Sedimentabschnitt meist durch pyritisierte Zellhohlräume charakterisiert.

Bemerkenswert ist der rasche Anstieg der Primärproduktion im Eckfelder Maar. Die Primärproduzenten werden in diesem Profilbereich jedoch ausschließlich durch die Kolonien der coccalen Chlorophycee Botryococcus repräsentiert. Mit knapp $1 \mathrm{Vol} . \%$ in Probe KS2 und knapp 2 Vol.\% in Probe KS3 dominiert die Alge deutlich das Gesamtinventar der organischen Partikel. Die zwischen $10 \mu \mathrm{m}$ und $40 \mu \mathrm{m}$ großen Kolonien sind vorwiegend isoliert und regellos im pyritreichen Sediment verteilt. Im Gegensatz zu den detritischen biogenen Partikeln kommen sie aber auch horizontiert angereichert vor und gehören somit zu den wenigen Schichtanzeigern in diesem Sedimentabschnitt.

\subsubsection{Mikrofazieszone 2b: Quarz-Huminit-Fazies}

Teufe:

E1/96: $37,17 \mathrm{~m}$ bis $35,91 \mathrm{~m}$

E3/96: $36,35 \mathrm{~m}$ bis $35,15 \mathrm{~m}$

Detail-Profil:

ÜS/QH-F1 (Anhang 1-1)

Dünn- und Stückschliffe:

ÜS/QH-F1.1 - 1.6 (Anhang 1-1)

Kornschliffe:

KS4 - KS6 (Anlage 2)

Tab. 08: Laminen-Typen der Mikrofazieszone 2b: Quarz-Huminit-Fazies (vgl. Abb. 11)

\begin{tabular}{lll}
\hline Nr. & \multicolumn{1}{c}{ Laminen-Typ (LT) } & \multicolumn{1}{c}{ Schichtgefüge } \\
\hline $2 b-1$ & Huminitreiche Tonlagen & eben bis wellig parallel \\
$2 b-2$ & Quarzsandführende Siltlagen & meist unterbrochen eben bis \\
& unterbrochen wellig parallel \\
$2 b-3$ & $\begin{array}{l}\text { Lagen von komponentengestützt eingelagerten } \\
\text { Quarzkörnern, gelegentlich extraklastenführend }\end{array}$ & meist eben parallel \\
\hline
\end{tabular}

Die Sedimente der MFZ 2b: Quarz-Huminit-Fazies zeichnen sich bereits makroskopisch durch eine markante Hell/Dunkel-Wechsellagerung im Submillimeter- bis Millimeterbereich aus (Anhang 1-2). Im Dünnschliff-Bild sind die hellen Lagen (LT 2b-3) als $50 \mu \mathrm{m}$ bis selten $1 \mathrm{~mm}$ mächtige, ungradierte bis allenfalls undeutlich normalgradierte Quarzkornschichten mit komponentengestütztem Korngefüge zu erkennen (Anhang 2-2, Fig. 01 und Fig. 02). Nur die mächtigeren Kornlagen führen gerundete Extraklasten in Feinsandkorngröße, die im Vergleich zu den Quarzen jedoch nur untergeordnet auftreten.

Die dunklen, bis $0,5 \mathrm{~mm}$ mächtigen Tonlagen ( $L T$ 2b-1) erscheinen makroskopisch weitgehend homogen. Im Dünnschliff ist jedoch eine feine, meist unterbrochene Internlamination zu erkennen. Sie wird durch laterale Anreicherungen von eckigen, überwiegend siltgroßen Quarzkörnern (LT 2b-2) hervorgerufen, die im allgemeinen jedoch nur 1 bis 3 Korndurchmesser mächtig sind (Anhang 2-2, Fig. 03).

Die tonreichen Schichten (LT 2b-1) führen den Hauptanteil der organischen Substanz innerhalb der Mikrofazieszone $2 \mathrm{~b}$. Abgesehen von wenigen Sporiniten, Lamalginiten, Bituminiten und Liptodetriniten, die in den Proben KS4 bis KS6 mit jeweils maximal 0,5 Vol.\% auftreten, wird 
das organische Partikel-Inventar eindeutig von detritischen Huminiten dominiert. Das Verhältnis von Huminit zu Liptinit erreicht in Probe KS4 mit über 15:1 seinen höchsten Wert innerhalb der gesamten Abfolge von E1/96 und E2/96. Bei den Huminiten handelt es sich hauptsächlich um siltkorngroße Humodetrinite, Detro-Gelinite und isoliert auftretende Corpohuminite. In etwas geringerem Maße sind eingeregelte Ulminite mit einer Länge von meist $<150 \mu \mathrm{m}$ am Schichtaufbau beteiligt.

Im Gegensatz zu der liegenden MFZ 2a: Quarz-Pyrit-Fazies wurde framboidaler Pyrit in diesem Profilbereich mit einer maximalen Häufigkeit von lediglich 1,6 Vol.\% (Probe KS5) nachgewiesen. Das Vorkommen von isolierten oder schichtweise angereicherten Framboiden ist dabei überwiegend an die dunklen organisch-reichen Laminen gebunden (Anhang 2-2, Fig 04).
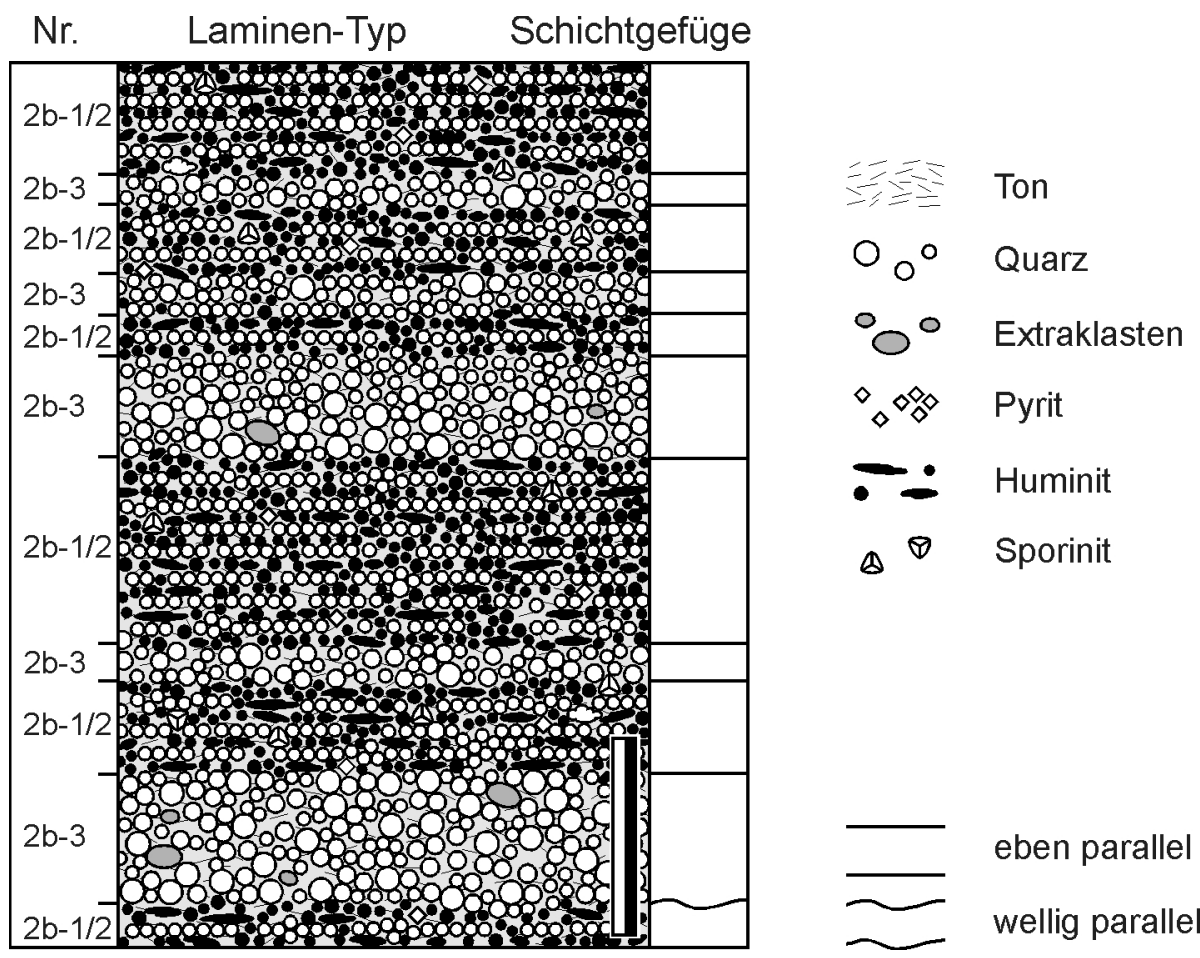

Komponenten

Schichtgefüge

Abb. 11: Laminen-Typen der Mikrofazieszone 2b: Quarz-Huminit-Fazies (Klassifizierung der Schichtgefüge nach COLE \& PICARD 1975).

\subsection{Lithofazieszone 3: Biogene Laminite}

Teufe:

E1/96: $33,15 \mathrm{~m}$ bis $5,00 \mathrm{~m}$

E2/96: 20,50m (Endteufe) bis $4,50 \mathrm{~m}$

E3/96: $32,65 \mathrm{~m}$ bis $4,25 \mathrm{~m}$

Die Lithofazieszone 3: Biogene Laminite entspricht weitgehend der von PIRRUNG (1998) ausgeschiedenen Lithozone "LZ D2: bituminöse Laminite“. Der Terminus "biogen" wird hier allerdings dem Begriff "bituminös" vorgezogen, um die organisch-reichen Sedimente der Lithofazieszone 3, den Minerogenen Laminiten an der Basis der untersuchten Sedimentsäule eindeutig gegenüberzustellen. Die Verwendung des Attributs "bituminös" zur Charakterisierung der organischen Substanz in unreifen Sedimenten ist vom organisch-petrologischen Standpunkt aus gesehen per se problematisch. Bitumen ist als Gesteinskomponente sowohl organisch- 
petrologisch (z.B. COOK 1982, JACOB 1989) als auch organisch-geochemisch (z.B. TISSOT \& WELTE 1984) eindeutig definiert. Aus geochemischer Sicht wird Bitumen als der mit organischen Lösungsmitteln extrahierbare Anteil der organischen Materie verstanden. In organisch-reichen Sedimenten tritt er jedoch gegenüber den unlöslichen Bestandteilen (Kerogen) meist stark in den Hintergrund (TISSOT \& Welte 1984, Selley 1985, ZIMmerLe 1995). Nach ZinK \& PÜtTMANN (1994) liegt die Extraktausbeute (Lösungsmittel: Methanol und Dichlormethan) in einer 2,45m mächtigen Sedimentsequenz zwischen den Leithorizonten KL 30 und KL 2,5 des Eckfelder Grabungsprofils (vgl. Anlage 1) im Bereich von 50mgExt/gC $\mathrm{C}_{\text {org }}$.

Festes Bitumen entsteht infolge des Bituminisierungsprozesses aus den lipoiden Ausgangsstoffen von Vitriniten bzw. Liptiniten und tritt vorwiegend fein verteilt in Gesteinen mit verhältnismäßig hohem Reifegrad auf (TEICHMÜLLER 1989, TAYLOR et al. 1998). Es ist mikroskopisch nachweisbar, wurde jedoch in keiner Probe aus den Eckfelder Profundalsedimenten beobachtet (siehe auch WEHNER et al. 1992). Neben der allgemein kräftig grüngelben Fluoreszenz von Sporinit, weist auch die durchschnittliche Vitrinit-Reflexion von 0,44\% (WEHNER et al. 1992) auf einen Reifegrad der Seeablagerungen noch unterhalb einer Bitumengenese hin. Eine deutliche Dominanz der ungeradzahligen über die geradzahligen n-Alkane belegt ebenfalls, daß es sich bei dem Eckfelder Tertiärvorkommen um relativ unreife, thermisch nicht beanspruchte Sedimente handelt (ZINK \& PÜTTMANN 1994). Insgesamt ist die in den Schwarzpeliten auftretende organischen Substanz zum weitaus überwiegenden Teil als Kerogen und nicht als Bitumen anzusprechen (siehe auch CLAUSING 2001).

Die Bezeichnung "biogen" hat zudem den Vorteil, daß sie auch anorganisch-biogene Partikel, wie beispielsweise Diatomeen-Frusteln, einschließt, die in zwei Mikrofazieszonen der Biogenen Laminite gesteinsbildend auftreten.

\subsubsection{Mikrofazieszone 3a: Huminit-Fazies}

Teufe:

E1/96: $33,15 \mathrm{~m}$ bis $31,80 \mathrm{~m}$

$\mathrm{E} 3 / 96: 32,65 \mathrm{~m}$ bis $31,38 \mathrm{~m}$

Detail-Profil:

BL/H-F1 (Anhang 1-3)

Dünn- und Stückschliffe:

$\mathrm{BL} / \mathrm{H}-\mathrm{F} 1.1-1.13$ (Anhang 1-3)

Kornschliffe:

KS7 - KS11 (Anlage 2)

Tab. 09: Laminen-Typen der Mikrofazieszone 3a: Huminit-Fazies (vgl. Abb. 12)

\begin{tabular}{cll}
\hline Nr. & \multicolumn{1}{c}{ Laminen-Typ (LT) } & Schichtgefüge \\
\hline $3 a-1$ & Organisch-reiche Tonlagen, sideritführend & eben parallel \\
$3 a-2$ & $\begin{array}{l}\text { Lagen von komponentengestützt eingelagerten } \\
\text { Quarzkörnern, gelegentlich extraklastenführend }\end{array}$ & meist eben parallel \\
$3 a-3$ & Extraklasten- und quarzführende Tonlagen, ungradiert & eben parallel bis wellig parallel \\
\hline
\end{tabular}

Wie in der Mikrofazieszone $2 \mathrm{~b}$ treten organische Partikel vorwiegend in den dunklen tonreichen Laminen (LT 3a-1) auf, die mit $1 \mathrm{~mm}$ bis $3 \mathrm{~mm}$ jedoch generell mächtiger sind als die organisch-reichen Horizonte im Liegenden. Damit ist eine deutliche Erhöhung des Mazeralgehaltes von durchschnittlich 9,5 Vol.\% in MFZ $2 b$ auf 29,5 Vol.\% in MFZ $3 a$ verbunden. Die 
Probe KS9 weist mit über 50 Vol.\% die höchste Mazeralkonzentration innerhalb der gesamten Abfolge der zentrumsnahen Seesedimente auf. Es dominieren kleine detritische HuminitPartikel, wie siltkorngroße Humodetrinite und Detro-Gelinite. Botryococcus ist maximal 1,7 Vol.\% in Probe KS10 am Sedimentaufbau beteiligt. Die $10 \mu \mathrm{m}$ bis $40 \mu \mathrm{m}$ großen Kolonien treten vorwiegend isoliert und regellos im Sediment verteilt auf. Selten sind sie in dichten $10 \mu \mathrm{m}$ bis $50 \mu \mathrm{m}$ mächtigen Lagen oder in lockeren Anreicherungen von maximal 150 $\mu \mathrm{m}$ Mächtigkeit konzentriert.

In den tonreichen Schichten (LT 3a-1) der Huminit-Fazies wurde das Auftreten von Siderit mikroskopisch nachgewiesen (Probe BL/H-F1.8). Das Fe-Karbonat kommt überwiegend in Form isolierter meist undeutlich begrenzter Linsen vor, die in Bezug auf ihren Gehalt an organischer Substanz kaum von den umgebenden Tonen abweichen (Anhang 2-2, Fig. 05). Nur in Einzelfällen treten mehrere $100 \mu \mathrm{m}$ bis wenige Millimeter lange Siderit-Linsen horizontweise konzentriert auf und bilden dann unregelmäßige, wellig parallele Laminen von maximal $200 \mu m$ Mächtigkeit.

Die ton- und sideritreichen Sedimentabschnitte werden in unregelmäßigen Abständen von $0,5 \mathrm{~mm}$ bis $1 \mathrm{~mm}$ mächtigen Lagen (LT 3a-3) unterbrochen, in denen feinsandgroße Devonklasten und Quarzkörner gehäuft auftreten. Diese sind vorwiegend Tonmatrix-gestützt eingelagert und generell nicht gradiert. Daneben kommen häufig schichtparallel eingeregelte HuminitPartikel (hauptsächlich Texto-Ulminite) vor, die mit einer Länge im Millimeterbereich deutlich größer sind als die Ulminite des Umgebungssediments (Anhang 2-2, Fig. 06). Die klastenführenden Horizonte sind makroskopisch kaum von klastenfreien Tonlagen (LT 3a-1) zu unterscheiden.

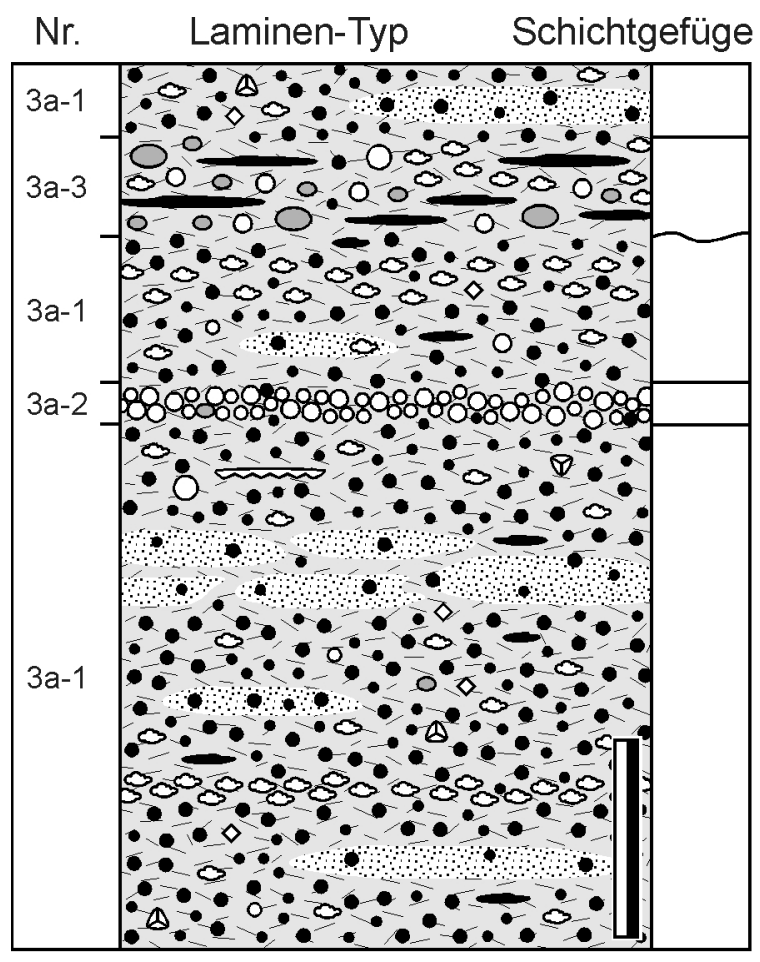

Komponenten

$\begin{array}{ll} & \text { Ton } \\ 00^{\circ} & \text { Quarz } \\ 0^{\circ} & \text { Extraklasten } \\ \diamond \diamond \diamond & \text { Pyrit }\end{array}$

- Siderit

- Huminit $0^{\infty} 0$ Botryococcus

$\Delta \nabla$ Sporinit $\infty$ Cutinit

Schichtgefüge

eben parallel

wellig parallel

Abb. 12: Laminen-Typen der Mikrofazieszone 3a: Huminit-Fazies (Klassifizierung der Schichtgefüge nach COLE \& PICARD 1975). 


\subsubsection{Mikrofazieszone 3b: Aulacoseira-Fazies}

Teufe:

E1/96: $31,80 \mathrm{~m}$ bis $27,90 \mathrm{~m}$

E3/96: $31,38 m$ bis $27,35 m$

Detail-Profil: $\quad$ BL/A-F1 (Anhang 1-4)

Dünn- und Stückschliffe: $\quad$ BL/A-F1.1 - 1.8 (Anhang 1-4)

Kornschliffe:

KS12 - KS16 (Anlage 2)

Tab. 10: Laminen-Typen der Mikrofazieszone 3b: Aulacoseira-Fazies (vgl. Abb. 13)

\begin{tabular}{lll}
\hline Nr. & Laminen-Typ (LT) & Schichtgefüge \\
\hline $3 b-1$ & Diatomeenlagen & eben parallel bis wellig parallel \\
$3 b-2$ & Organisch-reiche Tonlagen & eben parallel bis wellig parallel \\
$3 b-3$ & Klasten- und quarzführende Tonlagen, ungradiert & eben parallel bis wellig parallel \\
\hline
\end{tabular}

Die Mikrofazieszone 3b beginnt mit dem ersten mikroskopischen Nachweis von DiatomeenSchalen innerhalb der Profile E1/96 und E3/96. Abgesehen von vereinzelt auftretenden Formen der pennaten Gattungen Eunotia und Pinnularia wird die artenarme Diatomeen-Vergesellschaftung vom Indiviuenreichtum der zentrischen Kieselalge Aulacoseira cf. granulata dominiert (SCHILLER 1999). Im Bereich der Aulacoseira-Fazies erreichen Diatomeen-Frusteln Anteile von maximal 25 Vol.\% (Probe KS15) und stellen somit einen Hauptgemengteil des Gesteins dar. Die teils Gehäuseketten bildenden Kieselalgen sind in $30 \mu \mathrm{m}$ bis $1,5 \mathrm{~mm}$ mächtigen Lagen (LT 3b-1) angereichert (Anhang 2-2, Fig. 07). Sie heben sich durch ihren hellen Farbton bereits makroskopisch deutlich vom dunkleren Umgebungssediment ab. Zusammen mit monaxonen Skleren von Süßwasserschwämmen sind dispers verteilte Chrysophyceen-Zysten ein weiterer aber untergeordneter Bestandteil diatomeenreicher Lagen. In den Proben BL/A-F1.2 und BL/AF1.8 bilden die kieseligen Zysten jedoch $15 \mu \mathrm{m}$ bis $40 \mu \mathrm{m}$ mächtige, linsenförmige Massenansammlungen, die der Sedimentoberfläche als millimetergroße "patches" aufliegen. Die monospezifischen Zystenkonzentrationen treten typischerweise in geringmächtigen $(<40 \mu m)$ Tonhorizonten (LT 3b-2) zwischen zwei mächtigeren Diatomeenlagen auf, können lateral aber auch mit der Basis einer diatomeenreichen Schicht zusammenlaufen (Anhang 2-2, Fig. 08 bzw. Anhang 2-3, Fig. 01).

Innerhalb von Diatomeenlaminen (LT 3b-1) liegen die Theken von Aulacoseira cf. granulata in diagenetisch weitgehend unbeeinflußter Erhaltung vor. Daraus resultiert ein allgemein hohes Porenvolumen von Aulacoseira-Schichten in diesem Sedimentabschnitt (Anhang 2-3, Fig. 02). Lediglich im Grenzbereich zu den tonigen Zwischenlagen (LT 3b-2) sind Anzeichen für eine Überprägung opaliner Skelettsubstanz zu erkennen, die mit einem gehäuften Auftreten eisenreicher Mineralneubildungen einher geht. Vorwiegend an der Basis vieler Diatomeenlagen treten innerhalb von $10 \mu \mathrm{m}$ bis $30 \mu \mathrm{m}$ mächtigen Horizonten teils massenhaft Pyritframboide mit einem geringen Durchmesser von $<5 \mu$ m auf (Anhang 2-3, Fig. 03 und Fig. 04). Eine unter dem Auflichtmikroskop oftmals beobachtete, diffuse Rotfärbung in diesen Sedimentabschnitten ist nach EDX-Analysen hauptsächlich auf fein verteilten, partiell oxidierten Siderit zurückzuführen. Teilweise erscheinen auch die Aulacoseira-Frusteln unter Auflicht rostbraun verfärbt. Im BSE- 
Bild werden solche Schalenreste gegenüber unveränderten Kieselskeletten deutlich heller dargestellt (Anhang 2-3, Fig. 04). EDX-analytisch handelt es sich bei den überprägten Gehäusewänden um ein Stoffgemisch aus Eisen und Opal. Aufgrund der hohen Permeabilität bildeten Diatomeenlagen offensichtlich gute Wegsamkeiten für migrierende, eisenreiche Lösungen. An der Grenze zu den Tonschichten kam es dann zu einer Imprägnierung von Skelettopal und zur Neubildung eisenreicher Mineralphasen.

Aulacoseira-Lagen mit einer Mächtigkeit unterhalb von $500 \mu \mathrm{m}$ sind weitgehend frei von organischer Substanz. Dagegen führen mächtigere Diatomeenhorizonte sporadisch bis $200 \mu m$ lange Texto-Ulminite, die entweder regellos verteilt oder zusammen mit siltkorngroßen Humodetriniten, Detro-Geliniten und isolierten Corpohuminiten in tonreichen Linsen auftreten (Anhang 2-3, Fig. 05). Die tonigen Schlieren erreichen eine Länge von maximal $5 \mathrm{~mm}$ und sind mit $5 \mu \mathrm{m}$ bis selten $20 \mu \mathrm{m}$ vergleichsweise geringmächtig.

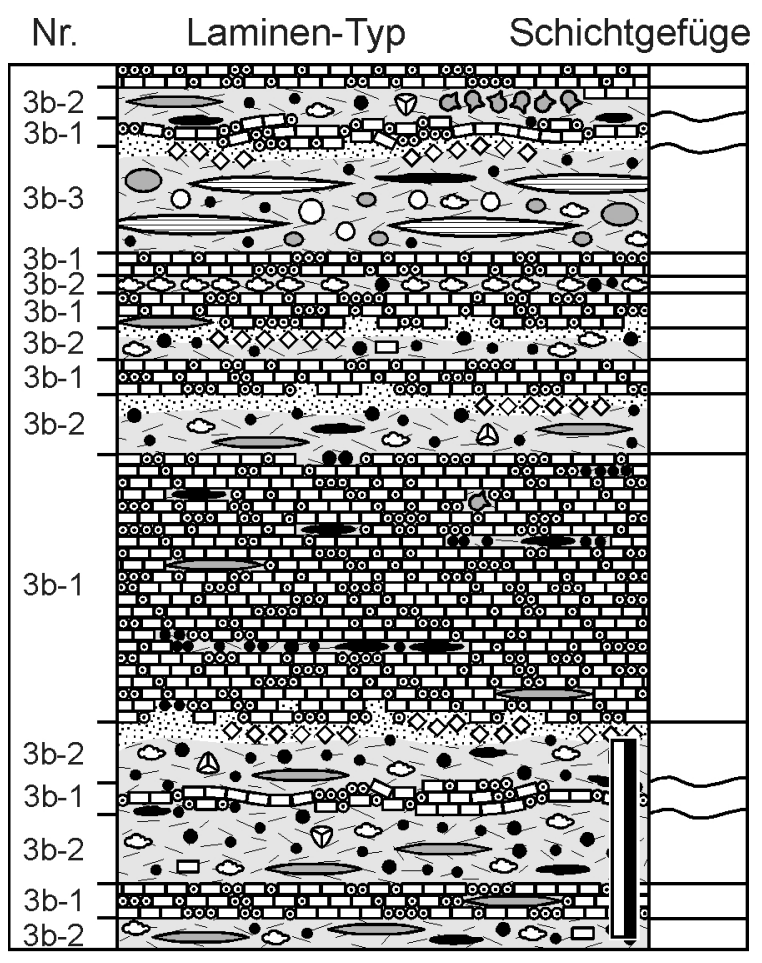

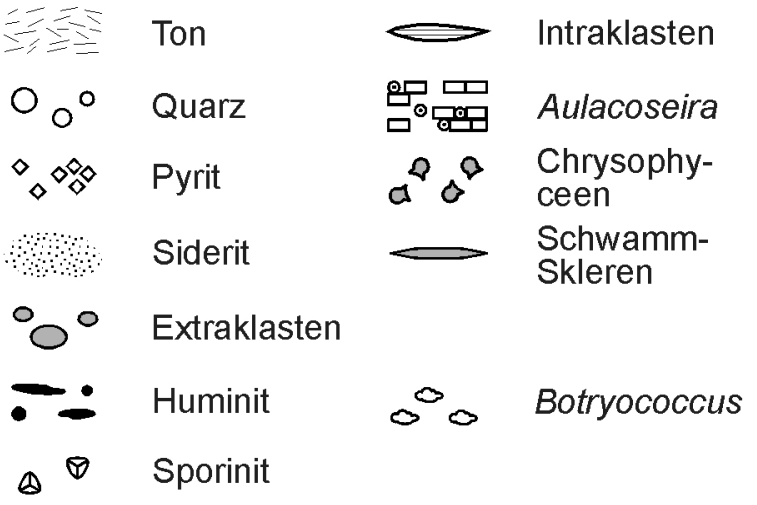

Schichtgefüge

Abb. 13: Laminen-Typen der Mikrofazieszone 3b: Aulacoseira-Fazies (Klassifizierung der Schichtgefüge nach COLE \& PICARD 1975).

Den weitaus überwiegenden Teil der organischen Substanz führen jedoch die zwischengeschalteten, $15 \mu \mathrm{m}$ bis $350 \mu \mathrm{m}$ mächtigen Tonlaminen (LT 3b-2). Im Gegensatz zu den Tonlinsen der Diatomeenlagen treten hier neben Huminiten auch Botryococcus-Kolonien mit regelmäßiger Häufigkeit auf (Anhang 2-3, Fig. 06). In den Proben BL/A-MF1.4 und BL/A-MF1.5 bilden sie in Form von 1 bis 3 Koloniedurchmesser starken Lagen gelegentlich auch die organi-

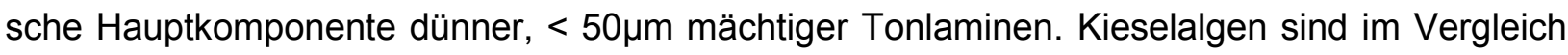
zu den diatomeenreichen Lagen nur sekundär an der Zusammensetzung organisch-reicher Tonschichten beteiligt. Den Hauptanteil kieseliger Komponenten bilden meist statistisch im Sediment verteilte Monaxone von Spongien (Anhang 2-3, Fig. 04).

Ähnlich wie im liegenden Profilabschnitt sind oftmals klasten- und quarzkornreiche Tonlagen (LT 3b-3) von < 1mm Mächtigkeit am Schichtaufbau beteiligt (Anhang 2-2, Fig. 07). Im Gegen- 
satz zu den Sedimenten der MFZ 3a: Huminit-Fazies bilden hier jedoch längliche LaminitFragmente einen charakteristischen Bestandteil der ungradierten Lagen (Anhang 2-3, Fig. 07). Die generell schichtparallel eingeregelten Fragmente bestehen zum überwiegenden Teil aus zusammenhängenden Gehäuseresten von Aulacoseira cf. granulata und erreichen eine Länge von mehreren $100 \mu \mathrm{m}$. Tonreiche Sediment-Fetzen sind im Dünnschliff dagegen nur undeutlich vom Umgebungssediment abzugrenzen. Mitunter beinhalten sie jedoch Massenansammlungen von Botryococcus und sind so fluoreszenzmikroskopisch eindeutig als Intraklasten identifizierbar.

Die Mikrofazieszone 3b schließt mit einem rund 2,65m mächtigen Rutschkörper (Typ-IIIResediment) ab, der in seinem mittleren Abschnitt intern gefaltete, teils zerscherte DiatomeenLaminite beinhaltet (vgl. Kap. 5.1.3).

\subsubsection{Mikrofazieszone 3c: Huminit-Opal-Fazies}

Teufe:

E1/96: $27,90 \mathrm{~m}$ bis $25,40 \mathrm{~m}$

$\mathrm{E} 3 / 96: 27,35 \mathrm{~m}$ bis $24,85 \mathrm{~m}$

Detail-Profil: $\quad$ BL/HO-F1 (Anhang 1-4)

Dünn- und Stückschliffe: $\quad$ BL/HO-F1.1 - 1.5 (Anhang 1-4)

Kornschliffe:

KS17 - KS22 (Anlage 2)

Tab. 11: Laminen-Typen der Mikrofazieszone 3c: Huminit-Opal-Fazies (vgl. Abb. 14)

\begin{tabular}{lll}
\hline Nr. & \multicolumn{1}{c}{ Laminen-Typ (LT) } & \multicolumn{1}{c}{ Schichtgefüge } \\
\hline $3 c-1$ & Organisch-reiche Opallagen & meist wellig parallel \\
$3 c-2$ & Organisch-reiche Tonlagen & meist wellig parallel \\
$3 c-3$ & Klasten- und quarzführende Tonlagen, ungradiert & eben parallel bis wellig parallel \\
$3 c-4$ & Sideritlagen & eben parallel bis wellig parallel \\
\hline
\end{tabular}

Die Sedimente der Mikrofazieszone 3c sind eine makroskopisch merkmalsarme Abfolge aus dunkelbraunen bis schwarzen Peliten. Eine Schichtung im Millimeter- bis Zentimeterbereich wird lediglich durch teils gradierte, siliziklastische Einschaltungen hervorgerufen (Anhang 1-4). Im mikroskopischen Bild sind die Ablagerungen der Huminit-Opal-Fazies durch dünne zwischen $5 \mu \mathrm{m}$ und 50 $\mathrm{mm}$ mächtige Opallaminen oder -linsen charakterisiert, die das tonreiche Sediment in netzartigen Strukturen durchziehen (LT 3c-1). Einzelne Linsen keilen gewöhnlich nach wenigen $100 \mu \mathrm{m}$ aus oder gehen lateral in weitere Opallaminen über (Anhang 2-4, Fig. 02 und Fig. 03). Häufig treten sie aber auch in $100 \mu \mathrm{m}$ bis selten $2 \mathrm{~mm}$ mächtigen Abschnitten konzentriert auf. In diesen Bereichen ist eine Abgrenzung diskreter Laminen kaum noch möglich (Anhang 2-4, Fig. 01). Im Rasterelektronenmikroskop erscheint die Opalsubstanz massiv, gelegentlich auch blättrig und ist typischerweise durch muschelige Bruchflächen gekennzeichnet. Unter dem Auflichtmikroskop wirken die opalinen Sedimentabschnitte meist farblos bis milchig weiß, können in der Umgebung von huminitischem Detritus aber auch auffällig rotbraun gefärbt sein (Anhang 2-4, Fig. 05). Die oftmals beobachtete Verfärbung im Kontaktbereich zu den Huminiten ist sehr wahrscheinlich auf eine Absorption mobiler Huminstoffe während der 
Opal-Präzipitation zurückzuführen. In den verfärbten Bereichen kleidet Opal ebenfalls die Risse und Hohlräume von größeren Ulminiten und Corpohuminit-Ansammlungen aus.

Quarz- und klastenführende Tonhorizonte, sind auch in diesem Sedimentabschnitt in einer Mächtigkeit von < $1 \mathrm{~mm}$ nachzuweisen (LT 3c-3). Sie führen eingeregelte, wenige 100 $\mu \mathrm{m}$ lange Laminit-Fragmente, die lithologisch eindeutig den ton- oder opalreichen Lagen im Liegenden und Hangenden zugeordnet werden können (Anhang 2-4, Fig. 04).

Daneben sind zwischen $10 \mu \mathrm{m}$ und $100 \mu \mathrm{m}$ mächtige sideritreiche Lagen und Linsen (LT 3c4) am Schichtaufbau der MFZ 3c beteiligt. Im BSE-Bild zeigen sie sich als lockere Ansammlungen von $2 \mu \mathrm{m}$ bis $8 \mu \mathrm{m}$ großen, meist xenomorphen Siderit-Einzelkristallen (Anhang 2-4, Fig. 06 und Fig. 07). Die mächtigeren Sideritlagen beinhalten häufig kleinere Tonlinsen und regellos verteilte Pflanzen-Fragmente (Huminite).

Innerhalb des Bohrprofils von E3/96 ist das erstmalige Auftreten von Ca-Phosphat aus der Probe BL/HO-F1.1 belegt. Die $2 \mu \mathrm{m}$ bis $6 \mu \mathrm{m}$ großen Apatit-Körner sitzen einem organischen Partikel von rund $100 \mu \mathrm{m}$ Durchmesser an, das aufgrund der Mineralumkrustung nicht näher identifiziert werden konnte.
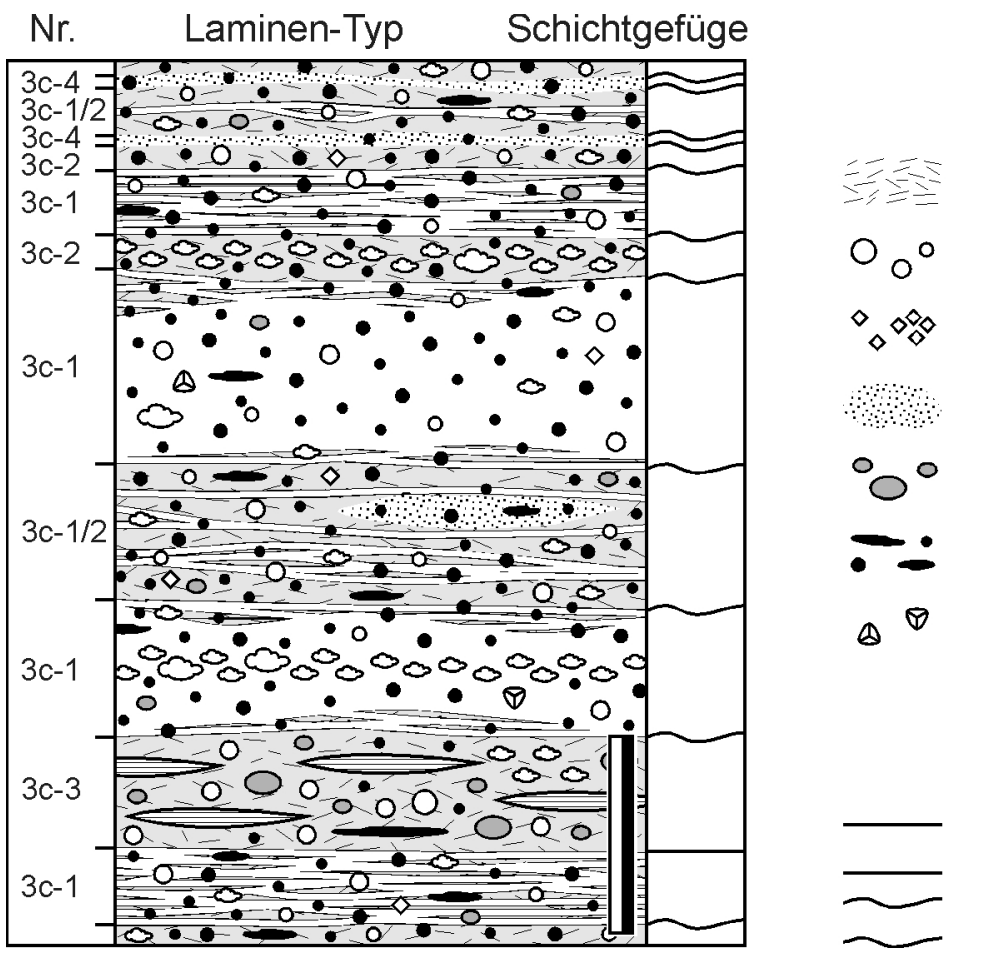

Ton

Quarz

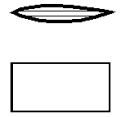

Intraklasten

Komponenten

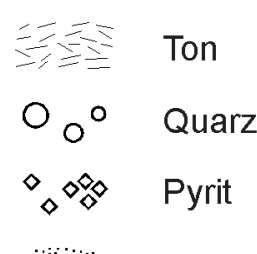

Siderit

Extraklasten

Huminit

$\infty^{\infty}$

Botryococcus

Sporinit

$\underline{\text { Schichtgefüge }}$

Abb. 14: Laminen-Typen der Mikrofazieszone 3c: Huminit-Opal-Fazies (Klassifizierung der Schichtgefüge nach COLE \& PICARD 1975).

Ein generelles Merkmal der Mikrofazieszone $3 c$ ist die gleichartige Verteilung der partikulären organischen Substanz, unabhängig von den lithologischen Grenzen. Sowohl in den opal(LT 3c-1) als auch in den tonreichen Sedimentabschnitten (LT 3c-2) treten organische Partikel in vergleichbarer Häufigkeit und Zusammensetzung auf. Damit unterscheiden sich die Sedimente der Huminit-Opal-Fazies deutlich von den angrenzenden Mikrofazieszonen. Bemerkenswert ist weiterhin der fehlende Nachweis von Botryococcus an der Basis der Folge (Probe KS17). Aus dem Ausfall von Primärproduzenten resultiert ein klares Übergewicht von huminitischem Detritus als Sedimentkomponente. Unter den Huminiten dominieren die kleinen, silt- 
korngroßen Partikel, wie Humodetrinite, Detro-Gelinite und isolierte Corpohuminite. Mit dem erneuten Häufigkeitsanstieg von Botryococcus auf mittlere Werte zwischen rund 3 Vol.\% bis 4 Vol.\% (Proben KS19 bis KS22) sinkt auch das Huminit:Liptinit-Verhältnis von knapp 14:1 an der Basis (Probe KS17) auf unter 2:1 am Top der Mikrofazieszone (Probe KS22). BotryococcusKolonien treten überwiegend isoliert und in statistischer Verteilung auf, bilden stellenweise aber auch unregelmäßige, oft unterbrochene Massenvorkommen von $50 \mu \mathrm{m}$ bis $100 \mu \mathrm{m}$ Mächtigkeit.

Das entscheidende Kriterium für die Abgrenzung der MFZ $3 c$ ist jedoch das vollständige Fehlen von Diatomeen. Im Gegensatz zu der liegenden und den hangenden Mikrofazieszonen konnten weder kieselige Gehäusereste noch Schalenabdrücke im feinkörnigen Sediment nachgewiesen werden. Den einzigen Beleg für das Auftreten kieseliger Mikrofossilien überhaupt bildet eine korrodierte Chrysophyceen-Zyste in Probe BL/HO-F1.4 (Anhang 2-11, Fig. 06). Das Aussetzten von Diatomeen im Hangenden des in Kap. 4.3.2 erwähnten Rutschkörpers ist ebenfalls aus der Bohrung von 1980 bekannt. Den diatomeenfreien Profilabschnitt beschreiben NEGENDANK et al. (1982, S. 168) als stark bituminösen Laminit mit "opal(?)-artigem Bindemittel". Weder das Rutschungsereignis, noch der fehlende Nachweis von Diatomeen oberhalb davon, sind also ein lokales Phänomen der Bohrungen von 1996, sondern kennzeichnen weite Bereiche der zentralen Seefazies.

\subsubsection{Mikrofazieszone 3d: Aulacoseira-Opal(CT)-Fazies}

Teufe:

E1/96: $25,40 \mathrm{~m}$ bis $19,17 \mathrm{~m}$

E3/96: $24,85 \mathrm{~m}$ bis $19,08 \mathrm{~m}$

Detail-Profil:

$\mathrm{BL} / \mathrm{AO}(\mathrm{CT})-\mathrm{F} 1$ (Anhang 1-5)

Dünn- und Stückschliffe:

$\mathrm{BL} / \mathrm{AO}(\mathrm{CT})-\mathrm{F} 1.1$ - 1.12 (Anhang 1-5)

Kornschliffe:

KS23 - KS33 (Anlage 2)

Tab. 12: Laminen-Typen der Mikrofazieszone 3d: Aulacoseira-Opal(CT)-Fazies (vgl. Abb. 15)

\begin{tabular}{|c|c|c|}
\hline $\mathrm{Nr}$ & Laminen-Typ (LT) & Schichtgefüge \\
\hline $3 d-1$ & Diatomeen/Opal(CT)-Lagen & $\begin{array}{l}\text { wellig parallel bis } \\
\text { unterbrochen wellig parallel }\end{array}$ \\
\hline $3 d-2$ & Organisch-reiche Tonlagen & meist wellig parallel \\
\hline $3 d-3$ & Klasten- und quarzführende Tonlagen, ungradiert & $\begin{array}{l}\text { eben parallel bis wellig } \\
\text { parallel }\end{array}$ \\
\hline $3 d-4$ & Sideritlagen & $\begin{array}{l}\text { eben parallel bis wellig } \\
\text { parallel }\end{array}$ \\
\hline
\end{tabular}

Nachdem in der liegenden Mikrofazieszone 3c keine Diatomeen nachgewiesen werden konnten, beginnt MFZ 3d mit dem erneuten Einsetzen von Aulacoseira cf. granulata. Im Gegensatz zu den Sedimenten der Aulacoseira-Fazies (MFZ 3b) sind die $10 \mu \mathrm{m}$ bis $250 \mu \mathrm{m}$ mächtigen diatomeenführenden Lagen (LT 3d-1) in diesem Profilabschnitt jedoch durch eine fortgeschrittene Umwandlung von ursprünglichem Skelettopal (Opal-A) hin zu Opal(CT) gekennzeichnet. Opal(CT) wurde rasterelektronenmikroskopisch in seiner für viele lakustrinen Tertiärvorkommen Mitteleuropas typischen Form (JANKOWSKI 1981, GOTH 1990, MÖRS 1995) als $1 \mu \mathrm{m}$ bis $3 \mu \mathrm{m}$ große Lepisphären nachgewiesen. Die kugeligen Opal-Aggregate sitzen vereinzelt oder in 
Gruppen den Innenseiten von Aulacoseira-Theken an. Auf den Monaxonen von Schwämmen wurden sie nicht beobachtet.

Der Grad der Opal-Diagenese kann sowohl zwischen Laminen unterschiedlicher Mächtigkeit als auch innerhalb einer einzigen diatomeenreichen Schicht stark variieren. Unter 50 $\mathrm{mm}$ mächtige Laminen sind gewöhnlich vollständig überprägt. Die Umrisse von monaxonen Schwammnadeln lassen sich jedoch vielfach immer noch nachweisen. Sie treten meist an den Schichtgrenzen oder in Assoziation mit eingelagerten Tonlinsen auf (Anhang 2-5, Fig. 05). Mächtigere Opallagen weisen oft eine gewisse Restporosität durch reliktisch erhalten gebliebene Diatomeen-Skelette auf (Anhang 2-5, Fig. 01). Die porösen Bereiche treten in unregelmäßigen Abständen innerhalb dichter, opalreicher Horizonte auf und sind durch eine hohe Zahl eisenhaltiger Gehäuserelikte geprägt (Anhang 2-5, Fig. 02). Offenbar wirkt sich die frühzeitige Adsorption von Eisen (vgl. Kap. 4.3.2) im Verlauf der Diagenese günstig auf das Erhaltungspotential von Skelettopal aus (vgl. Kap. 6.5.3). Der Übergang von porösen, gehäusereichen Abschnitten hin zu mikroskopisch dicht erscheinendem Opal ist typischerweise durch einen Wechsel in der Fluoreszenzfarbe von einem kräftigen hin zu einem blassen Grün verbunden. Stark eisenhaltige Schalenreste fluoreszieren hingegen nicht.

Neben dispers verteilten, $3 \mu \mathrm{m}$ bis $5 \mu \mathrm{m}$ großen Sideritkristallen führen Opallagen im Übergangsbereich zu den Tonschichten ( $L T$ 3d-2) häufig kleinere Huminite mit einer Länge von maximal $200 \mu \mathrm{m}$. Sie treten entweder isoliert oder zusammen mit Botryococcus, Liptodetrinit und Sporinit in organisch-reichen Tonschlieren auf. Die Schichtgrenzen zu den zwischengelagerten, $20 \mu \mathrm{m}$ bis selten $2 \mathrm{~mm}$ mächtigen Tonlaminen ( $L T 3 d-2$ ) sind in der Regel unscharf und meist wellig ausgebildet. Häufig gehen Opallagen sowohl vertikal als auch lateral in isolierte Linsen von variabler Länge über oder enden als diffuse opalreiche Schlieren im tonreichen Sediment (Anhang 2-5, Fig. 04 bis Fig. 06). Eine eindeutige Grenzziehung ist in diesen Fällen nicht möglich. Insgesamt sprechen alle Gefügemerkmale innerhalb der MFZ 3d für eine ausgeprägte Migration gelöster Kieselsäure (vgl. Kap. 6.5.3).

Auch die geringmächtigen $(<1 \mathrm{~mm})$, devonklastenführenden Tonlagen (LT 3d-3) beinhalten Opallinsen von wenigen $100 \mu \mathrm{m}$ Länge. Sie sind jedoch generell scharf begrenzt.

Ähnlich wie in der liegenden Mikrofazieszone 3c wurde Ca-Phosphat in Probe BL/AO(CT)F1.8 als ein rund 70um großes Aggregat von feinkörnigem Apatit nachgewiesen. Innerhalb einer siderit- und diatomeenreichen Lage umschließen die rund $5 \mu \mathrm{m}$ großen Einzelkörner einen ovalen organischen Partikel nahezu vollständig (Anhang 2-5, Fig. 03). Daneben bildet Apatit den Hauptgemengteil einer rund $6 \mathrm{~cm}$ mächtigen Konkretion, die bei einer Teufe von 21,9m die gesamte Breite der Bohrung E3/96 einnimmt (Probe BL/AO(CT)-F1.5). Das in derb-körnigen Massen oder in nadeligem Habitus auftretende Phosphat-Gemenge schließt in seinen Randbereichen verschieden große Laminit-Fragmente ein, die dem Umgebungssediment entsprechen (Anhang 1-5). Bei Bohrmeter 21,2 (E3/96) ist eine weitere Apatit-Konkretion von rund $3 \mathrm{~cm}$ Durchmesser Bestandteil eines $16 \mathrm{~cm}$ mächtigen Turbidits (Typ-III-Resediment, vgl. Kap. 5.1.3).

Innerhalb von Tonlagen (LT 3d-2) dominieren siltkorngroße Huminite (Humodetrinit, DetroGelinit und isolierte Corpohuminite) die allochthon-organische Sedimentkomponente. Botryococcus tritt mit einer Häufigkeit zwischen knapp $1 \mathrm{Vol}$ \% und rund $4 \mathrm{Vol}$ \% auf und ist vorwiegend dispers im Sediment verteilt. Die Koloniegröße schwankt im Bereich von $20 \mu \mathrm{m}$ und etwas über $100 \mu \mathrm{m}$. In der Zusammensetzung überlieferter Primärproduzenten bildet die Probe 
$\mathrm{BL} / \mathrm{AO}(\mathrm{CT})-\mathrm{F} 1.8$ eine Ausnahme. Hier wurden neben Botryococcus auch die dünnwandigen Einzelzellen einer kleinen, zwischen $8 \mu \mathrm{m}$ und $10 \mu \mathrm{m}$ großen Chlorophyceen-Form fluoreszenzmikroskopisch nachgewiesen. Sie sind nahezu ausschließlich in opalreichen Linsen erhalten geblieben. Innerhalb von opalfreien Tonhorizonten konnten dagegen so gut wie keine Zellreste identifiziert werden. Angesichts ihrer geringen Größe könnte es sich bei der Algen-Form um die coccale Chlorophycee Tetraedron handeln, die in höheren Profillagen (vgl. Kap. 4.3.5) eine bedeutende Stellung als Primärproduzent einnimmt. Der für Tetraedron charakteristische, kissenförmige Habitus ist an den stark verdrückten Individuen jedoch nicht zu belegen.

Opal(CT)-Lagen alternieren häufig mit $20 \mu \mathrm{m}$ bis $300 \mu \mathrm{m}$ mächtigen, sideritreichen Horizonten (LT 3d-4). Neben eisenreichen Schalenresten von Diatomeen führen Sideritlaminen häufig geringmächtige Opal(CT)-Linsen und undeutlich begrenzte Tonschlieren (Anhang 2-5, Fig. 06). Das Vorkommen von organischen Partikeln ist dagegen meist auf die tonreichen Abschnitte innerhalb sideritreicher Lagen und Linsen begrenzt.

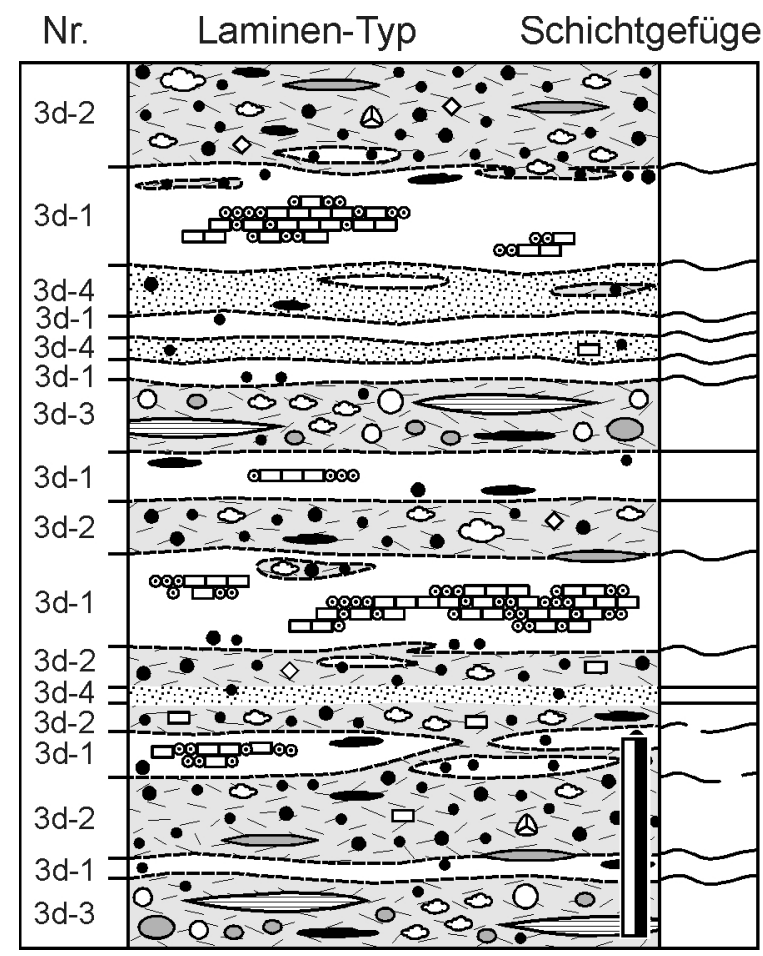

\begin{tabular}{|c|c|c|c|}
\hline$E=-5$ & Ton & $\Longleftrightarrow$ & Intraklasten \\
\hline $0_{0}^{\circ}$ & Quarz & 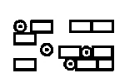 & Aulacoseira \\
\hline$>0$ & Pyrit & & Opal \\
\hline & Siderit & & $\begin{array}{l}\text { Schwamm- } \\
\text { Skleren }\end{array}$ \\
\hline & Extraklasten & & \\
\hline & Huminit & $0^{\circ}$ & Botryococcus \\
\hline & Sporinit & & \\
\hline
\end{tabular}

Abb. 15: Laminen-Typen der Mikrofazieszone 3d: Aulacoseira-Opal(CT)-Fazies (Klassifizierung der Schichtgefüge nach COLE \& PICARD 1975).

\subsubsection{Mikrofazieszone 3e: Tetraedron-Opal(CT)-Fazies}

Teufe:

E1/96: $19,17 \mathrm{~m}$ bis $5,00 \mathrm{~m}$

E2/96: $18,68 \mathrm{~m}$ bis $4,50 \mathrm{~m}$

E3/96: $19,08 \mathrm{~m}$ bis $4,25 \mathrm{~m}$

Detail-Profil:

$\mathrm{BL} / \mathrm{TO}(\mathrm{CT})-\mathrm{F} 1$ und BL/TO(CT)-F2 (Anhang 1-6)

Dünn- und Stückschliffe:

$\mathrm{BL} / \mathrm{TO}(\mathrm{CT})-\mathrm{F} 1.1-1.8$ und BL/TO(CT)-F2.1 - 2.7 (Anhang 1-6)

Kornschliffe:

KS34 - KS54 (Anlage 2) 
Die Grenze Mikrofazieszone 3d/3e wurde clusteranalytisch auf der Grundlage der Häufigkeitsverteilung von Primärproduzenten bestimmt. Sie liegt inmitten eines rund 1,2m mächtigen Profilbereichs zwischen dem ersten fluoreszenzmikroskopisch gesicherten Beleg von Tetraedron und dem starken Rückgang von Diatomeen-Gehäusen als Sedimentkomponente. Da sowohl der Nachweis von Tetraedron als auch die Häufigkeitsabnahme von DiatomeenSchalen aus einer fortschreitenden Opaldiagenese in den oberen rund $25 \mathrm{~m}$ der Profundalsedimente resultieren (siehe unten), markiert das Profilniveau zwischen der AulacoseiraOpal(CT)-Fazies und der Tetraedron-Opal(CT)-Fazies keinen primären Fazieswechsel. Die Grenze ist vielmehr Ausdruck einer diagenetisch bedingten Änderung in der Zusammensetzung der Mikrotaphozönose.

Tab. 13: Laminen-Typen der Mikrofazieszone 3e: Tetraedron-Opal(CT)-Fazies (vgl. Abb. 16)

\begin{tabular}{|c|c|c|}
\hline Nr. & Laminen-Typ (LT) & Schichtgefüge \\
\hline $3 e-1$ & Opal(CT)-reiche Horizonte & $\begin{array}{l}\text { wellig parallel bis unterbrochen wellig parallel, } \\
\text { meist mit unscharfen Schichtgrenzen }\end{array}$ \\
\hline $3 e-2$ & Tonreiche Horizonte & $\begin{array}{l}\text { wellig parallel bis unterbrochen wellig parallel, } \\
\text { meist mit unscharfen Schichtgrenzen }\end{array}$ \\
\hline $3 e-3$ & $\begin{array}{l}\text { Klasten- und quarzführende Tonlagen, } \\
\text { ungradiert }\end{array}$ & eben parallel bis wellig parallel \\
\hline $3 e-4$ & Sideritlagen & eben parallel bis wellig parallel \\
\hline
\end{tabular}

Der für die liegende Aulacoseira-Opal(CT)-Fazies typische Wechsel aus hellen, opalreichen und dunklen tonreichen Horizonten setzt sich auch im Sedimentabschnitt der TetraedronOpal(CT)-Fazies fort. Im Gegensatz zu MFZ 3d fällt jedoch bereits im makroskopischen Bild eine nach oben hin zunehmende Unschärfe der Schichtgrenzen und ein allgemein abnehmender Farbkontrast zwischen hellen und dunklen Lagen auf (Anhang 1-6). Daraus resultiert eine im Vergleich zu der liegenden Mikrofazieszone insgesamt hellere, graubraune Sedimentfarbe.

Im Dünnschliff ist eine Ausscheidung horizontbeständiger Laminen aufgrund der meist unregelmäßigen und unscharfen Schichtgrenzen kaum noch möglich (Anhang 2-5, Fig. 07 und Fig. 08). In vielen Fällen zeigen sich in vertikaler und lateraler Richtung graduelle lithologische Übergänge zwischen den opal- (LT 3e-1) und den tonreichen (LT 3e-2) Sedimentabschnitten. Lediglich einige mit $100 \mu \mathrm{m}$ bis $500 \mu \mathrm{m}$ verhältnismäßig mächtige Opal(CT)-Lagen sind scharf begrenzt. Sie zeigen im lateralen Verlauf allerdings oft starke Mächtigkeitsschwankungen

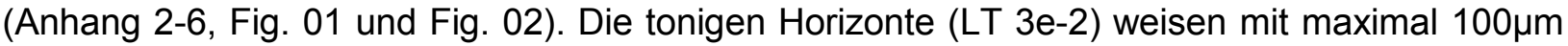
eine durchschnittlich geringere Mächtigkeit auf als in der liegenden Aulacoseira-Opal(CT)Fazies.

Innerhalb der MFZ 3e sind Diatomeen-Schalen nur reliktisch erhalten geblieben. Im BSE-Bild erscheinen sie aufgrund ihres oftmals erhöhten Eisengehaltes sehr viel heller als die sie umgebende Opalsubstanz. Eisenreiche Schalenreste wurden in der Bohrung E2/96 (Probe KS47) bis in eine Teufe von rund $9 \mathrm{~m}$ nachgewiesen. Größtenteils sind Diatomeen-Gehäuse jedoch nur indirekt über zahlreiche Abdrücke im opalreichen Sediment belegt. Gleiches gilt für die regellos im Sediment verteilten Monaxone von Spongien. Im unteren Abschnitt der TetraedronOpal(CT)-Fazies beinhalten die Hohlformen typischerweise Opal(CT)-Lepisphären (vgl. Kap.

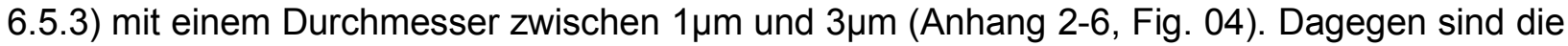


Schalen- und Sklerennegative in den oberen rund $5 \mathrm{~m}$ der Sedimentsequenz weitgehend frei von Opal (Anhang 2-6, Fig. 05). Unter dem Rasterelektronenmikroskop lassen sich statt dessen Schichtflächen nachweisen, die nahezu ausschließlich aus Lepisphären aufgebaut sind, aber keine Gehäuseabdrücke zeigen (Anhang 2-6, Fig. 06). Dieser Umstand dokumentiert die mehrfache Lösung und Wiederausfällung von Opal in diesem Profilbereich. Aus der Migration von Kieselsäure resultieren die diffusen Schichtgrenzen und die intensive Verkieselung primär ton- und organisch-reicher Sedimentabschnitte. Im BSE-Bild wird der hohe Grad der Opalisierung deutlich. Während sich im Dünnschliff BL/TO(CT)-F1.1 noch eine undeutliche Alternation von dunklen organisch-reichen Tonlaminen und hellen opalreichen Horizonten abzeichnet (Anhang 2-6, Fig. 03), sind primäre Schichtungsgefüge in höheren Profillagen (Probe BL/TO(CT)-F2.5) oftmals vollständig überprägt. Die ursprüngliche Schichtung ist in

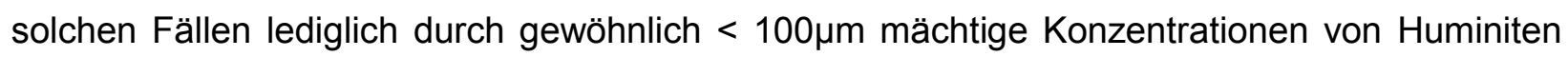
und Alginiten im opalreichen Sediment nachvollziehbar (Anhang 2-6, Fig. 07).

Das Huminit:Liptinit-Verhältnis innerhalb der MFZ 3e schwankt um niedrige Werte zwischen 2,8 in Probe KS34 und 0,1 in Probe KS44. Der ab der MFZ 3c zu beobachtende Trend hin zu einer kontinuierlichen Abnahme von organischem Detritus als Sedimentbestandteil erreicht damit seinen Höhepunkt. Sehr verstärkt wird diese Entwicklung durch die starke Präsenz von Tetraedron in diesem Profilbereich. Zwar scheint die dünnwandige Grünalge bereits abschnittsweise in der liegenden Aulacoseira-Opal(CT)-Fazies aufzutreten, doch ist ihr Nachweis durch die starke Kompaktion der Einzelzellen dort nicht gesichert (vgl. Kap. 4.3.4). In den intensiv verkieselten Sedimentabschnitten der Tetraedron-Opal(CT)-Fazies sind die gelblichgrün fluoreszierenden Tetraedron-Zellen dagegen häufig dreidimensional erhalten geblieben und durch ihren charakteristischen kissenförmigen Habitus eindeutig zu belegen (Anhang 2-12, Fig. 03). Sie treten häufig in disperser Verteilung auf, bilden sporadisch aber auch Massen-

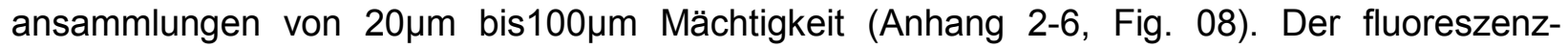
mikroskopische Nachweis von Tetraedron ist in erster Linie an den hohen Verkieselungsgrad der Sedimente gebunden, also vorwiegend aus taphonomischer Sicht zu bewerten. Schwach opalisierte Tonhorizonte führen kaum unverdrückte Exemplare. Statt dessen zeigen sich häufig diffus begrenzte und nur schwach grünlich bis rötlich fluoreszierende Partikel, die mit einer Länge von rund 10 $\mu \mathrm{m}$ den Dimensionen einer Tetraedron-Zelle entsprechen (Anhang 2-12, Fig. 05). Sie sind jedoch oft so stark kompaktiert, daß die Zuordnung zu Tetraedron zwar sehr wahrscheinlich, aber nicht zweifelsfrei gesichert ist. Allerdings bilden auch diese Partikel bis $100 \mu \mathrm{m}$ mächtige Massenvorkommen im Sediment (Proben KS40, KS41, KS43 - KS45, KS50, KS51, $\mathrm{KS} 53$, BL/TO(CT)-F1.1, BL/TO(CT)-F1.7, BL/TO(CT)-F2.3 - BL/TO(CT)-F2.7) die lateral in grünlich bis rötlich fluoreszierenden Lamalginit und Bituminit übergehen können (Anhang 2-12, Fig. 05). Tetraedron ist ebenfalls ein häufiger Bestandteil $20 \mu \mathrm{m}$ bis $100 \mu \mathrm{m}$ mächtiger Sideritlaminen und liegt hier vorwiegend in vollkörperlicher Erhaltung vor. Das Auftreten unkompaktierter Zellen hauptsächlich in verkieselten Sedimentabschnitten und sideritreichen Lagen gibt deutliche Hinweise auf den Zeitpunkt sowohl der Kieselsäuremigration als auch der Sideritgenese. Die Mineralneubildungen erfolgten offensichtlich frühdiagenetisch noch vor dem Beginn einer ausgeprägten Sedimentkonsolidierung. Von der fortschreitenden Verdichtung des Sediments waren vorwiegend tonreiche Horizonte betroffen, in denen die dünnwandige Alge heute nicht mehr sicher nachzuweisen ist. Als organische Komponente nur bedingt kompaktierbarer opal- und sideritreicher Lagen blieb Tetraedron dagegen häufig unverdrückt erhalten. 


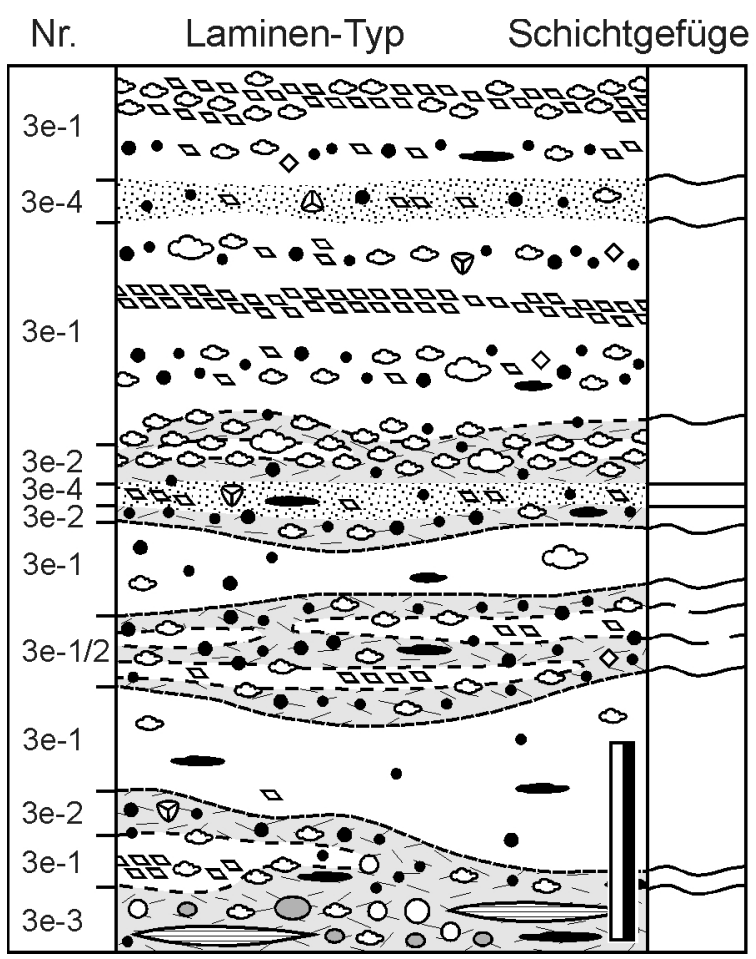

$\underline{\text { Komponenten }}$

$\begin{array}{llll} & \text { Ton } & 0^{\circ} \text { Extraklasten } \\ 0^{\circ} & \text { Quarz } & \text { Intraklasten } \\ \diamond \diamond & \text { Pyrit } & \text { Opal }\end{array}$

Siderit

- Huminit $0^{\infty}$ Botryococcus

(4) Sporinit Tetraedron

$\infty$ Cutinit

Schichtgefüge

Abb. 16: Laminen-Typen der Mikrofazieszone 3e: Tetraedron-Opal(CT)-Fazies (Klassifizierung der Schichtgefüge nach COLE \& PICARD 1975).

Neben Tetraedron ist Botryococcus ein häufig nachgewiesener Primärproduzent innerhalb der Tetraedron-Opal(CT)-Fazies und erreicht mit mehr als 7 Vol.\% in Probe KS44 den höchsten Häufigkeitswert in den Bohrungen E1/96 und E2/96. Wie in den liegenden Mikrofazieszonen sind die Kolonien vorwiegend statistisch im Sediment verteilt, bilden gelegentlich aber auch lockere Algenanreicherungen mit einer Mächtigkeit zwischen $20 \mu \mathrm{m}$ und selten $400 \mu \mathrm{m}$. Im Sediment überlieferte Massenproduktionen von Botryococcus und Tetraedron schließen sich keinesfalls gegenseitig aus. In Probe BL/TO(CT)-F1.1 sind beispielsweise alle Übergänge zwischen nahezu reinen Ansammlungen der jeweiligen Chlorophyceen-Form und Horizonten dokumentiert, in denen beide Algen-Gattungen zusammen konzentriert auftreten. Insgesamt zeigen ton- (LT 3e-2) und opalreiche Sedimentabschnitte (LT 3e-2) keine signifikanten Unterschiede in ihrer Mazeralzusammensetzung. Dieses kann nur als ein weiterer Hinweis darauf gewertet werden, daß die unscharfen Wechsel von opalreichen und opalärmeren Horizonten in vielen Fällen keine primären Schichtungsgefüge repräsentieren. Lediglich einige relativ scharf begrenzte und meist $>100 \mu m$ mächtige Opallagen sind verhältnismäßig arm an organischer Substanz (Anhang 2-6, Fig. 01 und Fig. 02).

\section{Resedimente}

Devonklastenreiche Ereignislagen haben insbesondere in den Lithofazieszonen 1 und 2 bedeutenden Anteil am Aufbau der Sedimente im Beckenzentrum. Demgegenüber sind die Schüttungsereignisse in Lithofazieszone 3 in erster Linie durch die Umlagerung von feinkörnigen Seesedimenten gekennzeichnet. Auf der Grundlage interner Gefügemerkmale sowie der Größe und Zusammensetzung der eingelagerten Komponenten lassen sich die 
Resediment-Sequenzen der zentralen Seefazies in diskrete Textur-Abschnitte unterteilen, die offensichtlich Variationen in den Transport- und Ablagerungsmechanismen der Sedimentströme widerspiegeln. Das Auftreten bzw. Fehlen definierter Abschnitte läßt Rückschlüsse auf das transportenergetisch wirksame Relief des Ablagerungsraumes zu und gibt Hinweise auf den Ursprungsort der Massenverlagerungen. Um eine Konfusion mit bestehenden Turbiditsequenzierungen, beispielsweise von BouMA (1962; Abschnitte $\mathrm{T}_{\mathrm{a}-\mathrm{e}}$ ) oder LOWE (1982; Abschnitte $\mathrm{R}_{2-3}$ und $\left.S_{1-3}\right)$, zu vermeiden, wurden für die Eckfelder Resedimente die Abschnittsbezeichnungen $\left(X_{1-2}, Y_{1-2}\right.$ und $\left.Z\right)$ verwendet. Auf Korrelationsmöglichkeiten mit BouMA- oder LoWE-Sequenzen wird im Einzelfall eingegangen.

Jeweils ideale Sequenzen beinhalten ein Maximum an Texturmerkmalen und Sedimentstrukturen. Sie sind in den Eckfelder Seeablagerungen jedoch nur in Resedimenten von über $10 \mathrm{~cm}$ Mächtigkeit verwirklicht. Geringmächtigere Ereignislagen zeigen gewöhnlich nur einen Teil der möglichen Textur-Abschnitte.

\subsection{Resediment-Sequenzen}

\subsubsection{Typ-I-Sequenzen}

Siliziklastische Einschaltungen vom Typ I sind in ihrem Auftreten auf die Lithofazieszone 1: Minerogene Laminite beschränkt und nehmen mit einer Mächtigkeit zwischen $2 \mathrm{~mm}$ und 1,4m rund $70 \%$ der Gesamtmächtigkeit der Folge ein. Die ideale Typ-I-Sequenz beinhaltet die TexturAbschnitte $X_{1}, Y_{1}$ und $Z$.

\section{Die ideale Typ-I-Sequenz}

Der Kontakt zwischen dem basalen $\mathrm{X}_{1}$-Abschnitt und den liegenden Laminiten ist generell nur geringfügig erosiv. Innerhalb einer Distanz von $2 \mathrm{~m}$ (Abstand zwischen den Bohransatzpunkten von E1/96 und E3/96) hat ein mit rund $50 \mathrm{~cm}$ Mächtigkeit überliefertes Schüttungsereignis (Teufe E1/96: $38,90 \mathrm{~m}$ bis $38,46 \mathrm{~m}$, Teufe E3/96: $38,36 \mathrm{~m}$ bis $37,86 \mathrm{~m}$ ) beispielsweise lediglich $2 \mathrm{~cm}$ Sediment erodiert. Vielfach überlagern grobklastische $\mathrm{X}_{1}$-Abschnitte die feinlaminierten Profilbereiche aber auch annähernd konkordant. In solchen Fällen werden die wellig ausgebildeten Kontaktflächen hauptsächlich durch "load casts" hervorgerufen (Anhang 27, Fig. 01). $X_{1}$-Abschnitte nehmen typischerweise mehr als $75 \%$ einer idealen Sequenzmächtigkeit ein. Entsprechend der geringen Erosionskapazität der Sedimentströme bilden laminierte Intraklasten nur einen seltenen Bestandteil des Komponentenspektrums. Als Hauptkomponente treten devonische Extraklasten in Grobsand- bis Steinkorngröße auf. Sie sind unorientiert in einer grobsilt- und feinsandreichen Pelitmatrix eingelagert. Der Matrixanteil ist jedoch vergleichsweise gering, so daß alle Übergänge zwischen einem vorwiegend matrix- und einem vorwiegend komponentengestützten Korngefüge auftreten können. Klasten bis Feinkieskorngröße sind meist gleichmäßig über den gesamten Abschnitt verteilt. Größere Devonkomponenten zeigen dagegen, zumindest in der oberen Hälfte von $\mathrm{X}_{1}$, eine deutliche Normalgradierung. Der Rundungsgrad variiert in der Regel zwischen eckig und gerundet und ist weniger abhängig von der Korngröße als vielmehr von der Lithologie der Klasten (vgl. Kap. 6.1.1). 


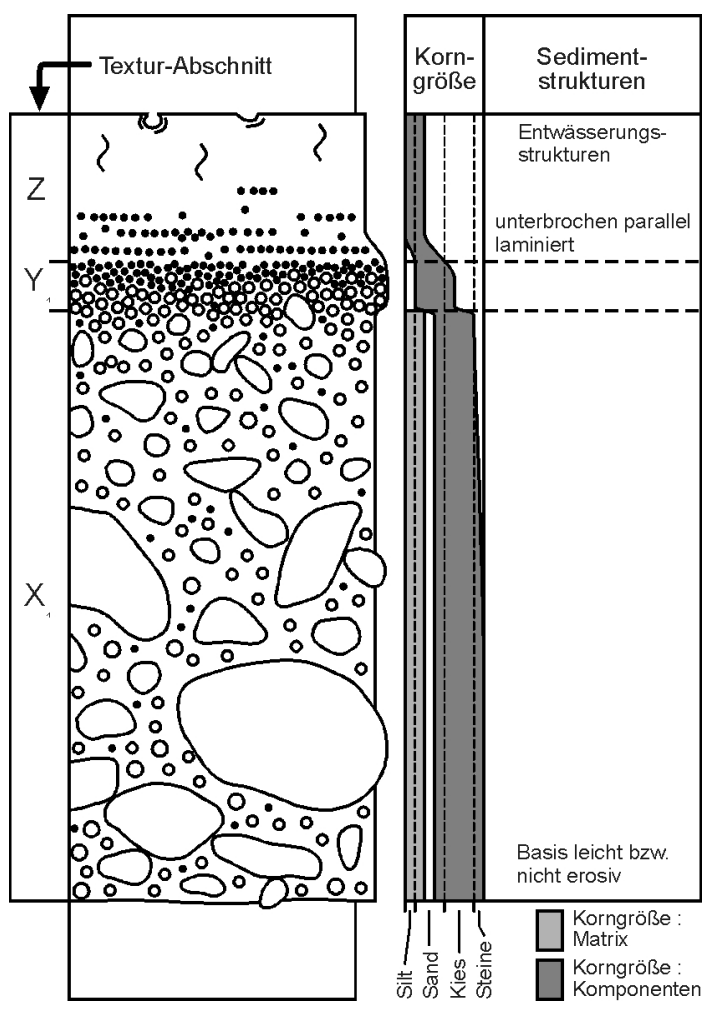

Abb. 17: Ideale Resediment-Sequenz vom Typ-I.

Der Texturwechsel zum folgenden $Y_{1}$-Abschnitt ist meist graduell und durch eine starke Abnahme des Matrixanteils im Übergangsbereich $X_{1} / Y_{1}$ gekennzeichnet (Anhang 2-7, Fig. 01). Innerhalb von $Y_{1}$ schwankt die Korngröße der Extraklasten meist zwischen Feinkies und Feinsand, obwohl gelegentlich größere Devonkomponenten im Mittelkiesbereich vertreten sein können. Aufgrund der vergleichsweise geringen Mächtigkeit von $\mathrm{Y}_{1}$-Abschnitten (rund 5\% der Gesamtmächtigkeit einer Resediment-Sequenz) ist eine Gradierung der Komponenten nicht immer deutlich entwickelt. Sandgroße Körner sind allerdings vorwiegend im obersten Teil konzentriert. Das Hauptmerkmal von $Y_{1}$ bleibt jedoch ein generell komponentengestütztes Korngefüge.

Der Abschnitt Z am Top einer idealen Typ-I-Sequenz besteht im wesentlichen aus korngestützt eingelagerten Ti-Oxiden und Quarzen in Silt- bis Feinsandkorngröße. Im unteren Drittel kann das feinkörnige Sediment allerdings gewisse Anteile an mittel- bis grobsandigen Extraklasten führen, die häufig in unterbrochenen eben-parallelen Lagen von etwa zwei Millimetern Mächtigkeit angereichert sind (Anhang 2-7, Fig. 02). Mit der raschen Häufigkeitsabnahme dunkler Devonklasten zur Hangendgrenze hin nimmt der quarzreiche Abschnitt eine einheitlich mittelgraue Sedimentfarbe an. In den oberen zwei Dritteln von Z treten Klasten nur noch untergeordnet und vorwiegend in Feinsandkorngröße auf. Sie bilden aber gelegentlich den Hauptbestandteil rund $1 \mathrm{~mm}$ dünner Lagen und Linsen, die sich nahe der Grenze zu den hangenden Laminiten in unscharf diffuse, wolkige Strukturen auflösen können (Anhang 2-7, Fig. 03). Diese sekundären Gefüge deuten auf eine Fluidisierung bzw. Homogenisierung des feinkörnigen Sediments infolge der postsedimentären Entwässerung hin. Fluidisierung ("fluidization", sensu LOWE 1975) tritt vorwiegend in grobsilt- und feinsandreichen Ablagerungen auf. Die Zugwirkung nach oben gerichteter Porenwasserströme führt bei dieser Korngrößenzusammensetzung oft zu einer Auflösung des ursprünglichen Korngefüges und somit zu einer Neuverteilung der Mineralkörner in diskreten, meist unscharf begrenzten Horizonten. Ein weiteres Merkmal aufsteigender Porenwässer sind unregelmäßige, steil geneigte Entwässerungskanäle von rund 2mm Durchmesser (Anhang 2-7, Fig. 03). Diese Art von Entwässerungsstrukturen bezeichnet LOWE (1975) als "fluidization channels" bzw. "type B pillars". Kennzeichnend für die in ZAbschnitten beobachteten Strukturen ist eine relative Anreicherung von dunklen Devonklasten im Zentrum der Kanäle, ein Umstand der offensichtlich aus der "Auswaschung" vorwiegend siltgroßer Quarzkörner durch den Porenwasserstrom resultiert. Fein- bis selten mittelsandgroße Devonklasten blieben als Residualsediment zurück und bilden den Hauptbestandteil der heutigen Kanalfüllung. Die Entwässerungskanäle sind nur wenige Zentimeter lang und scheinen nicht bis in die hangenden Laminite hineinzureichen. Allerdings zeichnen sich "Type B pillars" typischerweise durch einen stark irregulären Verlauf aus. Ihre in Schnitten senkrecht zur 
Schichtung erkennbaren Dimensionen entsprechen daher selten ihrer wahren Länge (LOWE 1975).

Z-Abschnitte sind generell scharf gegen die hangenden Laminite begrenzt. Entlang der Grenzfläche zeigen sich sporadisch aber rund $1 \mathrm{~cm}$ tiefe, taschenartige Einsenkungen von gröberkörnigem Hangendsediment (Anhang 2-7, Fig. 03). Die Einsackungen können im Sinne von LoWE (1975) als "load structures" interpretiert werden und sind die Folge einer inhomogenen Sediment-Fluidisierung. Eine partielle Mobilisierung von Silt- und Sandkörnern führte zu einem Massenverlust in isolierten Abschnitten des Grenzbereichs ("fluidized zones"), der durch das langsame Einsinken sandgroßer Devonklasten aus dem Hangenden kompensiert wurde.

\section{Abweichungen von der idealen Typ-I-Sequenz}

In Resedimenten mit einer Mächtigkeit unterhalb von $5 \mathrm{~cm}$ ist der basale Abschnitt $\mathrm{X}_{1}$ der idealen Sequenz generell nicht ausgebildet. In dieser Form entsprechen sie den von BULLWINKEL \& RIEGEL (2001) beschriebenen "Type A graded units". Siliziklastische Einschaltungen, die mit gradierten oder strukturlosen $\mathrm{Y}_{1}$-Abschnitten einsetzen sind an der Basis häufig durch das Auftreten von "flame structures" gekennzeichnet (Anhang 2-7, Fig. 04). Diese dokumentieren eine Intrusion von unverfestigtem Liegendsediment als Folge der erhöhten Auflast durch das Schüttungsereignis. Der Aufstieg von feinkörnigem Sediment geringerer Dichte ging notwendigerweise mit einer Abwärtsbewegung gröberer Komponenten aus dem unteren Bereich von $Y_{1}$ einher. Die stark welligen Basisgrenzen geringmächtiger Resedimente sind also nicht als Erosionsdiskordanzen anzusehen, sondern vielmehr das Resultat von "load cast". Den $Y_{1}$-Abschnitten liegen meist $<1 \mathrm{~cm}$ mächtige Z-Abschnitte mit auffallend scharfer Schichtgrenze auf (Anhang 2-7, Fig. 04). Sie sind vergleichsweise arm an Extraklasten und zeigen keine sichtbaren Anzeichen einer Sedimententwässerung. Bei unter $5 \mathrm{~mm}$ mächtigen Ereignislagen wird der Z-Abschnitt nur noch durch eine strukturlose, rund $1 \mathrm{~mm}$ mächtige Schicht von korngestützt eingelagerten Quarzen, Anatasen und untergeordnet feinsandgroßen Devonklasten vertreten. In Bezug auf das Korngefüge und die Mineralzusammensetzung entsprechen diese geringmächtigen Z-Lagen den klastenführenden Quarzkornlagen (LT 1a-5) der MFZ 1a: Quarz-Fazies (vgl. Kap. 4.1.1).

Unter $5 \mathrm{~mm}$ mächtige, oftmals nur undeutlich gradierte Typ-I-Resedimente tragen maßgeblich zum makroskopischen Eindruck einer ebenen Parallelschichtung innerhalb der Lithofazieszone 1: Minerogene Laminite bei. Durch die eingelagerten dunklen Devonklasten heben sich bereits $<1 \mathrm{~mm}$ mächtige Ereignislagen deutlich vom helleren Umgebungssediment ab (Anhang 1-1).

\subsubsection{Typ-II-Sequenzen}

Das Auftreten von phytoklastenführenden Typ-II-Sequenzen ist kennzeichnend für die Lithofazieszone 2: Übergangsschichten. Innerhalb der folgenden Mikrofazieszone 3a: HuminitFazies treten sie nur noch sporadisch und in geringen Mächtigkeiten von $<1 \mathrm{~cm}$ auf. Mit einem Resediment am Top der Lithofazieszone 2 erreichen Typ-II-Sequenzen eine maximale Mächtigkeit von 2,6m. In den meisten Fällen bleiben sie jedoch unterhalb von $3 \mathrm{~cm}$. Ideale Sequenzen beinhalten die Textur-Abschnitte $X_{1}, Y_{1}$ und $Z$. Sie sind in ihrer Gesamtheit jedoch nur in wenigen Ereignislagen vertreten. 


\section{Die ideale Typ-II-Sequenz}

Die basalen $\mathrm{X}_{1}$-Abschnitte stimmen in ihrer texturellen Ausbildung weitgehend mit den $\mathrm{X}_{1}$ Abschnitten von Typ-I-Sequenzen überein. Ähnlich wie diese greifen sie nur geringfügig erosiv in die liegenden Sedimente ein und zeigen im oberen Teil eine deutliche Normalgradierung der stein- bis mittelkiesgroßen, matrix- oder komponentengestützt eingelagerten Devonklasten (Anhang 2-7, Fig. 05). Eine bevorzugte Orientierung länglicher Komponenten ist nicht signifikant entwickelt. Der Übergang zum folgenden $Y_{1}$-Abschnitt vollzieht sich über eine kontinuierliche Abnahme des Matrixanteils in den obersten Zentimetern von $\mathrm{X}_{1}$.

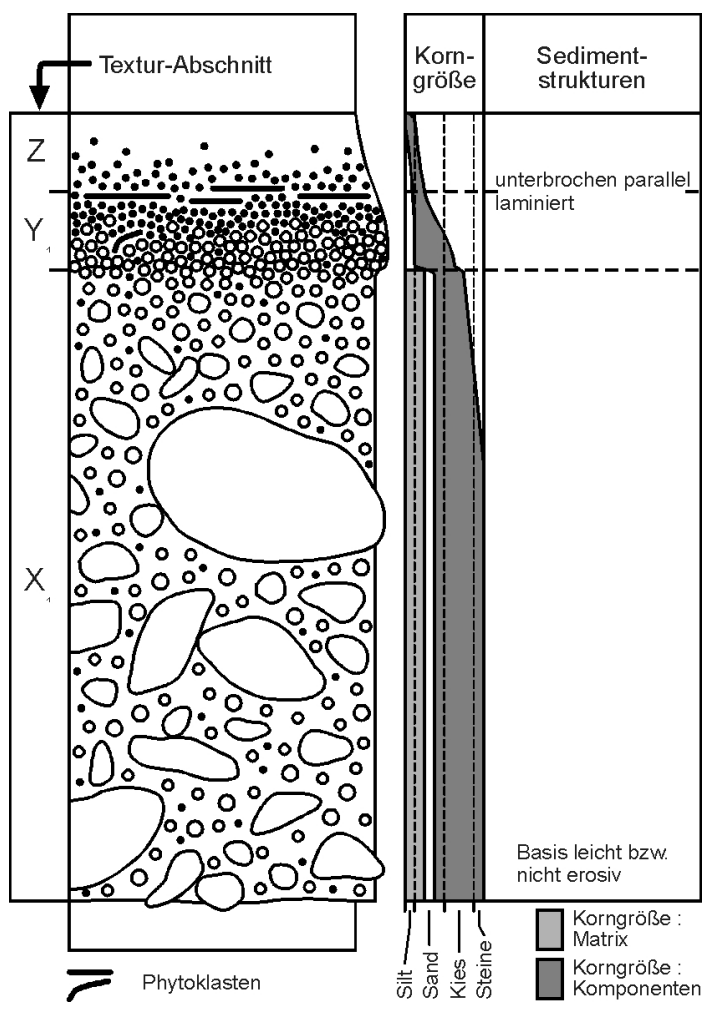

Abb. 18: Ideale Resediment-Sequenz vom Typ-II.

$\mathrm{Y}_{1}$-Abschnitte zeigen ein generell komponentengestütztes Korngefüge und eine allgemein deutliche Normalgradierung von Feinkies nach Feinsand als maximale Korngröße (Anhang 2-7, Fig. 05). Abweichend von den $\mathrm{Y}_{1}$-Abschnitten der Typ-I-Sequenzen können sie Pflanzen-Fragmente (Huminite) führen, die im Übergangsbereich zu den Z-Abschnitten typischerweise schichtparallel angereichert sind. Aus der Konzentration von Millimeter bis Zentimeter langen Phytoklasten und gelegentlichen Laminit-Fragmenten resultiert eine mehr oder weniger deutlich ausgeprägte, unterbrochene Parallellamination.

Im Gegensatz zu den Typ-I-Resedimenten zeigen die Z-Abschnitte von Typ-II-Sequenzen eine perfekte Normalgradierung von Feinsand nach Silt unter

Beteiligung von Quarzkörnern und gerundeten Devonklasten im unteren Teil des Abschnitts (Anhang 2-7, Fig. 05). Eingeregelte Huminite mit einer Länge im Millimeterbereich können eine untergeordnete Gesteinskomponente bilden.

\section{Abweichungen von der idealen Typ-II-Sequenz}

Geringmächtige $(<3 \mathrm{~cm})$ siliziklastische Einschaltungen werden nur durch $\mathrm{Y}_{1}$ - und ZAbschnitte vertreten und entsprechen den "Type B graded units" von BULLWINKEL \& RIEGEL (2001). Sie überlagern die Liegendsedimente konkordant oder mit nur wenig erosivem Kontakt (Anhang 2-7, Fig. 06). Bei unter $1 \mathrm{~cm}$ mächtigen Ereignislagen sind auch die $Y_{1}$-Abschnitte häufig nicht mehr signifikant ausgebildet. In solchen Fällen schließen die gradierten ZAbschnitte mit einer $<2 \mathrm{~mm}$ mächtigen, klastenarmen Lage von überwiegend siltgroßen Quarzkörnern ab. Die quarzreichen Toplagen von $Z$ erscheinen makroskopisch als auffallend helle Laminen und zeigen im mikroskopischen Bild alle texturellen Übergänge zu den meist ungradierten Quarzkornschichten (LT 2b-2 bzw. LT 2b-3) der MFZ 2b (vgl. Kap. 4.2.2). 


\subsubsection{Typ-III-Sequenzen}

Abweichend von den devonklastenreichen Resedimenten vom Typ I und II sind Typ-IIISequenzen durch das Auftreten laminierter Intraklasten als bedeutende Gesteinskomponente charakterisiert. Im Bereich der Lithofazieszone 3: Biogene Laminite bilden intraklastenreiche Resedimente mit einer Mächtigkeit zwischen wenigen Millimetern und maximal 2,65m den vorherrschenden Typ von Ereignislagen. In den rund 30m Sedimentsäule werden Mächtigkeiten über $10 \mathrm{~cm}$ allerdings nur von fünf Resedimenten diesen Typs erreicht. Die ideale Typ-IIISequenz ist mit den Abschnitten $X_{1}, X_{2}, Y_{1}, Y_{2}$ und $Z$ im mächtigsten von innen, innerhalb der Mikrofazieszone 3b: Aulacoseira-Fazies entwickelt (Teufe E1/96: 30,60m bis 27,95m, Teufe E3/96: $30,00 \mathrm{~m}$ bis $27,35 \mathrm{~m}$ ).

\section{Die ideale Typ-III-Sequenz}

Wie üblich beginnt die ideale Resediment-Sequenz mit einem nicht oder nur geringfügig erosiv eingreifenden $\mathrm{X}_{1}$-Abschnitt, der im Vergleich zu den idealen Typ-I- und Typ-II-Sequenzen jedoch deutlich geringmächtiger ist. Trotz eines gewissen Matrixanteils sind die stein- bis grobsandgroßen Devonklasten vorwiegend komponentengestützt eingelagert (Anhang 2-8, Fig. 01). Während grobsandige und feinkiesige Klasten nahezu gleichmäßig über den gesamten Abschnitt verteilt auftreten, kommen größere Komponenten vorwiegend nahe der Basis von $\mathrm{X}_{1}$ vor und implizieren so eine meist gut ausgebildete Normalgradierung. Die unorientiert eingelagerten Devonklasten zeigen prinzipiell alle Rundungsgrade. Unabhängig von der Korngröße sind eckige bis angerundete Komponenten jedoch weitaus am häufigsten vertreten.

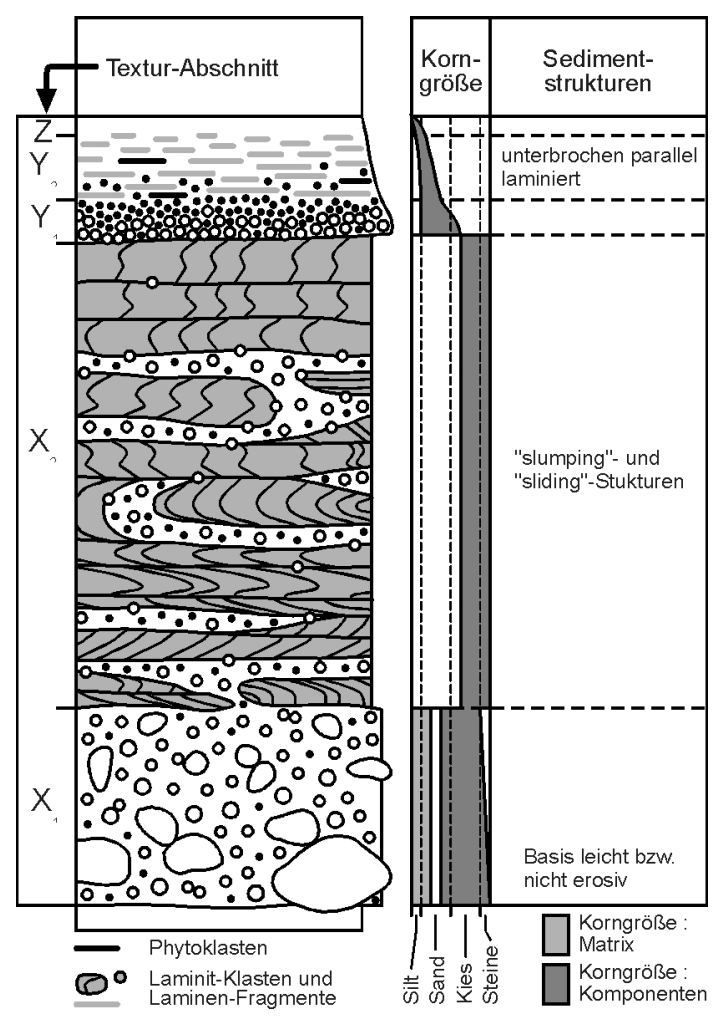

Abb. 19: Ideale Resediment-Sequenz vom Typ-III.

Mit unregelmäßiger aber scharfer Schichtgrenze folgt der intraklastenreiche Abschnitt $\mathrm{X}_{2}$ (Anhang 2-8, Fig. 02). $X_{2}$-Abschnitte nehmen rund $60 \%$ der Gesamtmächtigkeit einer idealen Typ-III-Sequenz ein und umfassen ein weitgehend ungeordnetes Gemenge aus intern gefalteten Laminit-Bruchstücken und umgelagerten Resedimenten. Rutschfaltung ("slumping") im Meso- und Makrobereich ist ein häufiges Gefügemerkmal dieses Abschnitts und betrifft sowohl die geschichteten Pelite als auch die gröberklastischen Einschaltungen. In vielen Fällen sind die brekziierten Laminite durch horizontale oder flach geneigte Rutschungsflächen ("slip faces", EINSELE 1991) gegeneinander abgegrenzt. Dabei zeigen längliche Intraklasten oftmals eine Einregelung senkrecht oder in steilem Winkel zu ihrer internen Feinschichtung (Anhang 2-8, Fig. 03). Steingroße Laminitklasten sind üblicherweise mehrfach horizontal zerschert. Der jeweilige Versatz beträgt allerdings nur wenige Zentimeter. 
Den $\mathrm{X}_{2}$-Abschnitten liegen mit scharfer Schichtgrenze die devonklastenreichen $\mathrm{Y}_{1}$-Abschnitte auf. Sie sind in der Regel deutlich von Feinkies nach Mittel- oder Feinsand gradiert und zeichnen sich durch ein generell komponen-tengestütztes Korngefüge aus. Im Basisbereich von $\mathrm{Y}_{1}$ können kiesgroße Intraklasten eine seltene Gesteinskomponente bilden. Der Übergang zum folgenden Textur-Abschnitt $Y_{2}$ vollzieht sich in der Regel graduell über eine kontinuierliche $A b-$ nahme des Devonklastenanteils (Anhang 2-8, Fig. 04). Feinsandgroße Extraklasten und Quarzkörner kommen in regelloser Verteilung zwar auch in höheren Bereichen von $Y_{2}$ vor, sind im Vergleich zum liegenden Abschnitt jedoch deutlich seltener vertreten.

Kennzeichnend für $\mathrm{Y}_{2}$-Abschnitte ist eine von der Basis zum Top hin zunehmende Häufigkeit dünner, lediglich ein bis zwei Laminen starker Laminit-Fragmente und länglicher Phytoklasten (Ulminite). Blattkutikeln (Cutinite) sind gemessen an den pelitischen Laminiten im Liegenden und Hangenden der Resedimente oftmals häufiger vertreten. Mit Blick auf schichtparallele Sedimentanbrüche zeigen die Laminit-Fetzen meist eine unregelmäßig ovale Form. Ihre generelle Einregelung führt zu einer unterbrochenen Internlamination in $\mathrm{Y}_{2}$ und ist ein charakteristisches Texturmerkmal dieses Abschnitts. Ebenso wie die schichtparallel orientierten Phytoklasten zeigen auch die Laminit-Fragmente eine deutliche Normalgradierung durch eine Längenabnahme von mehreren Millimetern an der Basis bis hin zu wenigen 100 $\mu \mathrm{m}$ am Top des Abschnitts (Anhang 2-8, Fig. 08). Im obersten Bereich von $Y_{2}$ können feinsandgroße Quarzkörner

gehäuft auftreten. Daraus resultiert ein oftmals gradueller Übergang zum folgenden, quarzreichen Abschnitt $Z$ am Top der Resediment-Sequenz.

Z-Abschnitte sind durch eine perfekte Normalgradierung von feinsand- nach siltgroßen Quarzkörnern gekennzeichnet. Intra- und Extraklasten kommen in diesem vergleichsweise geringmächtigen Abschnitt nicht mehr vor. Innerhalb der Mikrofazieszone 3b: AulacoseiraFazies können die Gehäuse-Bruchstücke von Diatomeen jedoch einen wesentlichen Bestandteil von $\mathrm{Z}$ bilden.

\section{Abweichungen von der idealen Typ-III-Sequenz}

Eine annähernd vollständige Typ-III-Sequenz wird durch ein rund $85 \mathrm{~cm}$ mächtiges Resediment innerhalb der Mikrofazieszone 3e: Tetraedron-Opal(CT)-Fazies vertreten (Teufe E2/96: $14,75 \mathrm{~m}$ bis $13,95 \mathrm{~m}$, Teufe E3/96: $14,95 \mathrm{~m}$ bis 14,10m). Ohne einen zwischengeschalteten, devonklastenreichen $Y_{1}$-Abschnitt geht hier ein rund $50 \mathrm{~cm}$ mächtiger $X_{2}$-Abschnitt graduell in einen nur $2 \mathrm{~cm}$ mächtigen $\mathrm{Y}_{2}$-Abschnitt über.

Die weitaus überwiegende Zahl von Typ-III-Ereignislagen überschreitet Mächtigkeiten von $5 \mathrm{~cm}$ jedoch nicht und entspricht den von BULLWINKEL \& RIEGEL (2001) beschriebenen "Type C graded units". Sie beinhalten lediglich die obersten drei Abschnitte $\left(Y_{1}, Y_{2}\right.$ und $\left.Z\right)$ der idealen Sequenz (Anhang 2-8, Fig. 05). Extraklastenreiche $Y_{1}$-Abschnitte sind oftmals nur in geringer Mächtigkeit von wenigen Millimetern ausgebildet und führen neben sandigen Devonklasten häufig auch große Mengen an länglichen, schichtparallel eingeregelten Ulminiten. $Y_{2}$-Abschnitte nehmen in den meisten Fällen über ${ }^{2} / 3$ der Gesamtmächtigkeit ein und enthalten Laminit-Fragmente, die petrographisch eindeutig den jeweiligen Peliten im Liegenden und Hangenden des Resediments zugeordnet werden können (siehe auch MINGRAM 1998, Tab. 1). Makroskopisch läßt sich die für $Y_{2}$ charakteristische Internlamination am besten innerhalb der Mikrofazieszone 
3b: Aulacoseira-Fazies und 3d: Aulacoseira-Opal(CT)-Fazies durch das Auftreten heller diatomeenreicher Laminit-Fragmente nachvollziehen.

Die auffallend hellen Z-Abschnitte geringmächtiger Typ-III-Sequenzen bilden eine Reihe von Leithorizonten (KL 2.5, Ka-LH, KL 30, KF9 und KSG 30) innerhalb des Eckfelder Grabungsareals (vgl. Anlage 1). Ka-LH und KL 30 führen neben Quarz als Hauptbestandteil (60 Gew.\% bis 85 Gew.\%) auch Gehalte an Kaolinit (8 Gew.\% bis 25 Gew.\%) und Illit/Muskovit (3 Gew.\% bis $15 \mathrm{Gew}$ \%). Dabei ist insbesondere bei Ka-LH ein deutlicher Anstieg der Tonmineralkomponente von insgesamt 15 Gew.\% auf 40 Gew.\% zum Top hin zu beobachten (LUTZ 1993a). Sowohl LUTZ (1993a) als auch MINGRAM (1994, 1998) deuten die hellen kaolinitführenden Leithorizonte als die obersten Abschnitte von Turbidit-Sequenzen. Gelegentlich sind bei Typ-IIIResedimenten weder die extraklastenführenden $\mathrm{Y}_{1}$-Abschnitte noch die quarzreichen ZAbschnitte deutlich ausgeprägt. In solchen Fällen werden die Ereignislagen ausschließlich aus umgelagerten Laminit-Fragmenten (Abschnitt $Y_{2}$ ) aufgebaut, die häufig nur im Dünnschliff von den ungestörten Laminiten im Liegenden und Hangenden zu unterscheiden sind. Beim Unterschreiten einer Mächtigkeit von etwa $2 \mathrm{~mm}$ ist auch eine Normalgradierung der Intraklasten kaum noch entwickelt. In dieser strukturlosen Form entsprechen $\mathrm{Y}_{2}$-Abschnitte den ungradierten, intraklastenführenden Tonlagen (LT 3b-3, LT 3c-3, LT 3d-3 und LT 3e-3), die einen charakteristischen Laminen-Typ innerhalb der Lithofazieszone 3: Biogene Laminite darstellen (vgl. Kap. 4.3).

\subsection{Transport- und Ablagerungsmechanismen}

\section{$\mathrm{X}_{1}$-Abschnitte}

Bei Resedimenten, die durch einen feinkörnigen Matrixanteil gekennzeichnet sind, stellt sich grundsätzlich die Frage nach dem Transportmechanismus der gröberklastischen Gesteinskomponenten. Auf der Grundlage ihres rheologischen Verhaltens lassen sich Dichteströme prinzipiell in kohäsive ("laminar", FISHER 1971, ENOS 1977, MIDDLETON 1993; "plastic", LOWE 1979, Postma 1986; "cohesive", MULDER \& AlEXANDER 2001) und nicht-kohäsive ("turbulent", FISHER 1971, ENOS 1977, MIDDLETON 1993; "fluidal", LOWE 1979, POSTMA 1986; "frictional", MULDER \& ALEXANDER 2001) Ströme differenzieren.

Elektrochemische Anziehungskräfte (Kohäsion) bilden sich vorwiegend zwischen Partikeln in Ton- und Feinsiltkorngröße aus (FISHER 1971). Nach LOWE (1975) zeigen Sedimente mit einer mittleren Korngrößenzusammensetzung unterhalb von etwa $50 \mu \mathrm{m}$ einen signifikanten kohäsiven Zusammenhalt. Die Festigkeit einer feinkörnigen, kohäsiven Matrix ("matrix strengh", NARDIN et al. 1979) ist zusammen mit dem Porenwasserdruck und dem Auftrieb ("buoyancy") größerer Klasten der Haupttransportmechanismus in "debris flows" (HAMPTON 1975, LEEDER 1982, INESON 1985). Infolge des vorwiegend laminaren Transports größerer Komponenten in kohäsiven Sedimentströmen und ihrer spontanen en masse Ablagerung durch "cohesive freezing" (EINSELE 1991, MULDER \& ALEXANDER 2001) sind Debrite im Allgemeinen durch die Abwesenheit interner Sedimentstrukturen und eine schlechte, vertikale Sortierung der Korngrößen bzw. durch eine inverse Gradierung charakterisiert (FISHER 1971, SMITH 1986, EINSELE 1991, MARR et al. 2001, MULDER \& ALEXANDER 2001). Die vorwiegend matrixgestützten Komponenten (FISHER 1971, SMITH 1986, EINSELE 1991) zeigen oft eine Einregelung annähernd parallel zur Fließrichtung des Sedimentstromes (FISHER 1971, ENOS 1977, EINSELE 1991). 
Abweichungen in bezug auf eine Normalgradierung von Debriten und eine regellose Orientierung länglicher Klasten werden jedoch beispielsweise von ENOS (1973), LEEDER (1982) und EINSELE (1991) beschrieben.

Innerhalb von $\mathrm{X}_{1}$-Abschnitten ist der Matrixgehalt vergleichsweise gering und durch teils bedeutende Grobsilt- und Feinsandanteile gekennzeichnet. Größere Komponenten sind oft korngestützt und generell regellos eingelagert. Weiterhin zeigt die Normalgradierung, insbesondere im oberen Bereich von $X_{1}$, eine zumindest partielle Korngrößensortierung während des Sedimenttransports an. Die Gesamtheit der lithologischen Merkmale macht ein plastisches Fließen durch die Tragkraft einer kohäsiven Matrix als einzig wirksamen Transportmechanismus unwahrscheinlich. MULDER \& ALEXANDER (2001) weisen darauf hin, daß "debris flows" in turbulente hyperkonzentrierte Dichteströme ("hyperconcentrated density flows") übergehen können, sofern sie ausreichende Gehalte an Matrixkörnern führen, die größer sind als Mittelsilt (siehe auch HAMPTON 1972, 1975 und FISHER 1971). Der Übergang von einem vorwiegend laminaren (kohäsiven) hin zu einem vorwiegend turbulenten (nicht-kohäsiven) Sedimenttransport ("flow transformation", FISHER 1983) findet dabei häufig an steilen Hängen statt, da hohe Transportgeschwindigkeiten die Entwicklung interner Turbulenzen begünstigen (FISHER 1983, MULDER \& ALEXANDER 2001). Im Gegensatz zu "debris flows" werden Klasten in "hyperconcentrated density flows" überwiegend durch Korn-zu-Korn-Kollisionen ("dispersive pressure", MULDER \& ALEXANDER 2001) in Schwebe gehalten. Ein Auftrieb größerer Komponenten innerhalb der Sediment-Wasser-Matrix ("matrix buoyant lift", LOWE 1982) kann ein zusätzlicher Transportmechanismus sein. Gleitbewegungen ("rafting", MULDER \& ALEXANDER 2001) treten häufig auf und transportieren große Gesteinsblöcke über teilweise beträchtliche Distanzen hinweg. Die generell hohen Sedimentkonzentrationen verhindern jedoch im allgemeinen eine signifikante gravitative Korngrößensortierung. Lediglich im oberen Teil zeigen die Ablagerungen oft eine deutliche Normalgradierung als Folge einer kontinuierlichen Sedimentverdünnung durch den Zutritt von Umgebungswasser im Frontbereich des Dichtestroms (MULDER \& ALEXANDER 2001).

$\mathrm{X}_{1}$-Abschnitte greifen typischerweise nicht oder nur geringfügig erosiv in die liegenden Laminite ein. Die geringe Erosionskapazität resultiert aus dem Prozeß des "hydroplaning", der kennzeichnend ist für subaquatische "debris flows", aber auch für schnell fließende, turbulente Dichteströme (ENOS, 1977, IVERSON 1997, MARR et al. 2001, MULDER \& ALEXANDER 2001).

Auf der Grundlage der oben geführten Diskussion werden $\mathrm{X}_{1}$-Abschnitte als die Ablagerungen von "hyperconcentrated density flows" (sensu MULDER \& ALEXANDER 2001) interpretiert. Allerdings sind vertikale und laterale Übergänge zu Debriten nicht auszuschließen (siehe auch LOWE 1982). "... different parts of a flow may be classified in different ways, and flow transformation can occur in both time and space." (MULDER \& ALEXANDER 2001, S. 278.)

\section{$\mathrm{X}_{2}$-Abschnitte}

$\mathrm{X}_{2}$-Abschnitte treten nur in den beiden mächtigsten (2,65m bzw. 0,85m) Resedimenten der Lithofazieszone 3: Biogene Laminite auf und bilden dort den jeweils mächtigsten TexturAbschnitt. Die eingelagerten Laminit-Bruchstücke sind oftmals durch annähernd horizontalparallele Scher- und Rutschungsflächen ("slip faces") begrenzt. Zusammen mit dem häufigen Auftreten von Rutschfaltung ("slumping") zeigen die internen Gefügemerkmale einen vorwie- 
gend durch Rutsch- und Gleitbewegungen geprägten Sedimenttransport an. Dabei wurde die primäre Lamination der Intraklasten zwar verformt, aber nicht komplett zerstört.

Die in $\mathrm{X}_{2}$-Abschnitten überlieferten Sedimente können als Rutschungsbrekzien angesprochen werden und sind das Resultat von "subaquatic slides" bzw. "slumps" (EINSELE 1991).

\section{Y1-Abschnitte}

Innerhalb idealer Typ-I- und Typ-II-Sequenzen entwickeln sich devonklastenreiche $\mathrm{Y}_{1}$ Abschnitte kontinuierlich aus den liegenden $X_{1}$-Abschnitten durch eine graduelle Abnahme des Matrixanteils in den obersten Bereichen von $X_{1}$. Die generell komponentengestützten Klasten zeigen oft eine deutliche Normalgradierung von Feinkies nach Mittel- oder Feinsand. Lediglich wenige geringmächtige $Y_{1}$-Abschnitte innerhalb der Lithofazieszone 1: Minerogene Laminite sind mitunter strukturlos ausgebildet.

Insbesondere bei steilen Hangneigungen können hyperkonzentrierte Dichteströme durch die kontinuierliche Aufnahme von Umgebungswasser in weniger dichte, aber nach wie vor konzentrierte Suspensionsströme übergehen ("concentrated density flows", MULDER \& ALEXANDER 2001). Nach LOWE $(1982,1988)$ und MULDER \& ALEXANDER (2001) werden größere Mengen an Feinkies und grobkörnigem Sand nur in Suspensionsströmen transportiert, deren Sedimentkonzentration groß genug ist, um ausreichend viele Korn-zu-Korn-Kollisionen zu erlauben. Der daraus resultierende, ungerichtete Druck ("dispersive pressure") hält die Komponenten in Suspension und ist ein grundlegender Transportmechanismus in "concentrated density flows". Mit kontinuierlichem Wasserzutritt können Flüssigkeitsturbulenzen jedoch zunehmend an Bedeutung für den Sedimenttransport gewinnen und zu einer signifikanten Sortierung der Korngrößen führen. Der Grad der Wasseraufnahme ist in direktem Maße abhängig von der Geschwindigkeit des Sedimentstroms. Verlangsamt der Strom seine Geschwindigkeit unter einen kritischen Wert, beispielsweise am Grund des Sedimentationsbeckens, treten intergranulare Reibungskräfte in den Vordergrund und verursachen so eine schnelle Sedimentakkumulation ("frictional freezing", MULDER \& ALEXANDER 2001). "Frictional freezing" ist offenbar auch die Ursache für die generell scharfen Obergrenzen geringmächtiger $Y_{1}$-Abschnitte in den Typ-I-Sequenzen der Lithofazieszone 1: Minerogene Laminite. Im Falle von Typ-II- und Typ-IIIResedimenten setzten vermutlich die häufig eingelagerten Phytoklasten im oberen Bereich von $Y_{1}$ den Reibungswiderstand zwischen den mineralischen Komponenten soweit herab, daß hier graduelle Übergänge zu den hangenden Z- bzw. $Y_{2}$-Abschnitten vorherrschen (Anhang 2-7, Fig. 05 und Fig. 06 bzw. Anhang 2-8, Fig. 04 und Fig. 05).

Die $Y_{1}$-Abschnitte können den Ablagerungen von "concentrated density flows" (sensu MULDER \& ALEXANDER 2001) zugeordnet werden. In ihrer Korngrößenzusammensetzung (Feinkies bis Feinsand) und der häufigen Normalgradierung ähneln sie den $\mathrm{S}_{3}$-Abschnitten von "highdensity turbidity currents" (LOWE 1982, 1988) bzW. den $T_{a}$-Abschnitten der idealen BouMASequenz (BOUMA 1962). Die von LOWE beschriebenen Entwässerungsstrukturen treten allerdings erst in den hangenden Z-Abschnitten von Typ-I-Sequenzen auf.

Konzentrierte Dichteströme können eine hohe Erosionskapazität aufweisen (MULDER \& ALEXANDER 2001). Im Falle der vergleichsweise geringmächtigen Resedimente der zentralen Eckfelder Seefazies ist eine Erosion von Laminiten jedoch häufig nur indirekt, beispielsweise durch das Auftreten intraklastenreicher $\mathrm{Y}_{2}$-Abschnitte im Hangenden von $\mathrm{Y}_{1}$, überliefert. 


\section{$\mathrm{Y}_{2}$-Abschnitte}

$\mathrm{Y}_{2}$-Abschnitte treten ausschließlich in den Typ-III-Sequenzen der Lithofazieszone 3: Biogene Laminite auf und gehen typischerweise aus gradierten, extraklastenreichen $\mathrm{Y}_{1}$-Abschnitten hervor. Der Wechsel vollzieht sich über eine kontinuierliche Abnahme von Devonkomponenten bzw. eine Zunahme eingeregelter Laminitintraklasten. In $\mathrm{Y}_{2}$ nimmt die Größe der Laminit-Fragmente wie auch die der länglichen Phytoklasten zum Top hin deutlich ab. Normalgradierte, extraklastenreiche $Y_{1}$-Abschnitte gehen in Typ-III-Resedimenten also graduell in normalgradierte, intraklastenreiche $Y_{2}$-Abschnitte über. Auffällig ist weiterhin das gehäufte Auftreten feinsandgroßer Quarzkörner im obersten Bereich vieler $\mathrm{Y}_{2}$-Abschnitte.

Die deutliche Normalgradierung der Laminit-Fragmente läßt auf eine kontinuierliche, durch gravitative Sortierungsprozesse gekennzeichnete Ablagerung aus einer Sedimentsuspension schließen. Dabei lag ihre Sedimentationsgeschwindigkeit offensichtlich zwischen der von kiesbis sandgroßen Devonklasten (Abschnitt $Y_{1}$ ) und der von feinsand- bis siltgroßen Quarzkörnern (oberster Bereich von $Y_{2}$ und Abschnitt $Z$ ). Es ist jedoch zu erwarten, daß das hydrodynamische Verhalten der scheibenförmigen Laminit-Fragmente stark von dem der körnigen Partikel abwich. Zum Zeitpunkt der Ablagerung von $\mathrm{Y}_{2}$-Abschnitten hatte der Suspensionsstrom bereits seine grobkörnige Fracht verloren ( $Y_{1}$-Abschnitt), war also verhältnismäßig gering konzentriert. Der durch einen Zutritt von Umgebungswasser gekennzeichnete Transport in "concentrated density flows" (vgl. Y1-Abschnitte) läßt zusätzlich ein erhöhtes Ausströmen von Porenwasser während der Ablagerung von $Y_{1}$ und eine weitere Verdünnung der fließenden Sedimentsuspension darüber erwarten. Dies führt zu der Vorstellung, daß der Mechanismus des Intraklastentransports im wesentlichen durch ein Gleiten der plattigen Laminit-Fragmente im aufsteigenden Porenwasserstrom dominiert war. Dabei wurden sie nach ihrer Größe sortiert und weitgehend parallel zur Fließrichtung des Stroms eingeregelt. Nur im Grenzbereich zwischen der Sedimentsuspension und dem umgebenden Seewasser traten signifikante Turbulenzen auf, die zu der gleichzeitigen Ablagerung von kleineren Laminit-Fragmenten und sandgroßen Quarzkörnern im obersten Teil von $Y_{2}$-Abschnitten führten.

Gradierte, intraklastenreiche Sedimentabschnitte treten ebenfalls in den mitteleozänen Seeablagerungen von Messel auf und werden von GOTH (1990) als "Turbiditische Ölschieferlagen" bezeichnet. Er deutet sie unter Vorbehalt als "Thixotropic Mud Slides" (sensu HSÜ \& KELTS 1985) oder als ein Resultat stehender Wellen (Seiches) und geht dabei von einem geringen lateralen Sedimenttransport "vielleicht im Bereich von nur wenigen Metern" aus (GoTH 1990, S. 36). Diese Interpretation kann nicht auf die $Y_{2}$-Abschnitte von Eckfeld übertragen werden. Im Gesamtzusammenhang der idealen Typ-III-Sequenzen gesehen sind sie eindeutig ein Bestandteil von Suspensionsströmen, die sich sukzessive - typischerweise über das hydrodynamische Zwischenstadium der konzentrierten Dichteströme (vgl. Y Y Abschnitte) - aus subaquatischen Rutschungen entwickelten (vgl. $\mathrm{X}_{2}$-Abschnitte).

Ein subrezentes Beispiel intraklastenreicher Turbidite beschreibt LUDLAM (1974) aus dem Fayetteville Green Lake (New York, USA). Die turbiditartigen Lagen treten nahe der steilen Randbereiche des Sees auf und bestehen vorwiegend aus mehr oder weniger horizontal eingeregelten Laminit-Fragmenten. Da die randlichen Seesedimente ebenfalls durch zahlreiche Rutschfaltungen gekennzeichnet sind, führt LUDLAM die Bildung von intraklastenreichen Turbiditen auf subaquatische "slumps/slides" zurück: "... in some cases slumps and slides intermix 
with water to become turbidity flows." (LUDLAM 1974, S. 660.) Diese Schlußfolgerung kann durch die vorliegenden Ergebnisse nur bestätigt werden. Gröberklastische, gradierte Turbidite (vergleichbar mit $Y_{1}$-Abschnitten) entstehen nach LUDLAM (1974), wenn neben feinkörnigen Laminiten auch bereits abgelagerte Resedimente in den erneuten Rutschungsprozeß einbezogen sind. Letzteres ist in $\mathrm{X}_{2}$-Abschnitten eindeutig der Fall. Ein Uferabbruch am Züricher Sees aus dem Jahre 1875 liefert ein weiteres Beispiel für die Entwicklung von Trübeströmen aus subaquatischen Rutschungsereignissen. Während die eigentliche Rutschung am Fuß des $20^{\circ}$ steilen Seehangs zum Stillstand kam, breitete sich der entstandene Suspensionsstrom über den gesamten zentralen Seeboden aus (HSÜ \& KELTS 1985).

\section{Z-Abschnitte}

Die Z-Abschnitte von Typ-I-Sequenzen (Lithofazieszone 1: Minerogene Laminite) bestehen vorwiegend aus silt- bis feinsandgroßen Quarzkörnern und siltkörnigen Anatasen. Sie zeigen allenfalls eine undeutliche Gradierung von Silt und Feinsand an der Basis zu vorwiegend Silt am Top des Abschnitts. In Typ-II- und Typ-III-Sequenzen treten Tonminerale, vorwiegend Kaolinit (LUTZ 1993a), als Gesteinsbestandteil hinzu. Diese Erweiterung des Korngrößenspektrums führt zu einer weitgehend perfekten Normalgradierung von Feinsand/Silt nach Silt/Ton.

Partikel, die kleiner sind als Mittelsand können ausschließlich durch Flüssigkeitsturbulenzen transportiert werden (LOWE 1982, 1988). Eine gut entwickelte Normalgradierung ist dabei typisch für die Ablagerungen eines Turbulenz-dominierten Suspensionsstroms (MULDER \& ALEXANDER 2001).

Die feinkörnigen Z-Abschnitte werden den Ablagerungen von "turbidity currents" (sensu MULDER \& ALEXANDER 2001) bzW. von "low-density turbidity currents" (sensu LOWE 1982) zugeordnet und entsprechen weitgehend den strukturlosen oder gradierten $T_{e}$-Abschnitten von BoumA (1962). Lediglich die idealen Typ-I-Resedimente zeigen an der Basis von $Z$ eine unterbrochene Parallel-Lamination durch die Anreicherung von mittel- bis grobsandigen Extraklasten, die im Sinne von BoumA (1962) als $T_{b}$-Abschnitt interpretiert werden kann.

Innerhalb der Mikrofazieszone 1a: Quarz-Fazies bilden ungradierte, gelegentlich extraklastenführende Lagen von komponentengestützten Silt- und Feinsandquarzkörnern einen häufigen Laminen-Typ (LT 1a-5). Sie können genetisch den Z-Abschnitten distaler Turbidite zugeordnet werden. Der laterale Übergang von normalgradierten Turbiditen hin zu ungradierten Silt/Sandlagen ist ebenfalls aus dem Crater Lake in Oregon (USA, NELSON 1967) sowie aus dem Brienzer See (Schweiz, STURM \& MATTER 1978) bekannt.

\section{Zusammenfassung}

Alle idealen Resediment-Sequenzen der zentralen Eckfelder Seefazies dokumentieren eine vertikale Entwicklung der Sedimentströme hin zu immer niedrigeren Sedimentkonzentrationen. Die Dichte der Ströme bestimmt dabei maßgeblich den Transport- und Ablagerungsmechanismus der mitgeführten Komponenten. In "hyperconcentrated density flows" ( $\mathrm{X}_{1}$ Abschnitte) dominieren Korn-zu-Korn-Interaktionen und der Auftrieb der Klasten innerhalb einer feinkörnigen Sediment-Wasser-Matrix den Partikeltransport. Mit der fortschreitenden Aufnahme von Umgebungswasser im Frontbereich des Dichtestroms und der kontinuierlichen Ablagerung der jeweils grobkörnigsten Fracht nimmt die Sedimentkonzentration immer weiter ab. In 
"concentrated density flows" ( $Y_{1}$-Abschnitte) sind Korn-zu-Korn-Kollisionen für den Transportmechanismus nach wie vor signifikant, werden aber zunehmend durch Flüssigkeitsturbulenzen ersetzt. Ist sowohl die Konzentration als auch die Korngröße der transportierten Partikel soweit erniedrigt, daß die Ablagerung aus einem Turbulenz-dominierten Suspensionsstrom erfolgt, gehen "density flows" bzw. "high-density turbidity currents" in "turbidity flows" bzw. "low-density turbidity currents" über (LOWE 1982, EINSELE 1991, MULDER \& ALEXANDER 2001). Unter diesen hydrodynamischen Bedingungen können sich BouMA-Sequenzen entwickeln, von denen der $\mathrm{T}_{\mathrm{a}}$ Abschnitt allerdings noch den "concentrated density flows" (Abschnitt $Y_{1}$ ) zugeordnet werden muß (MULDER \& ALEXANDER 2001). Da die folgenden Z-Abschnitte im allgemeinen zu geringmächtig sind, um komplette BouMA-Seqenzen auszubilden, ist am Top der Eckfelder Resedimente meist nur der $\mathrm{T}_{\mathrm{e}}$-Abschnitt von BouMA (1962) vertreten.

In bezug auf den Transportmechanismus bilden die $\mathrm{X}_{2}$ - und $\mathrm{Y}_{2}$-Abschnitte von Typ-IIISequenzen eine Ausnahme. Während die Massenverlagerungen im Falle von $\mathrm{X}_{2}$-Abschnitten durch "slumping/sliding"-Prozesse gekennzeichnet sind, wurde der in $\mathrm{Y}_{2}$-Abschnitten dokumen-

tierte Transport von Laminit-Fragmenten maßgeblich durch ihre flächige Form und ihre geringe Dichte beeinflußt.

Insgesamt sind die basalen X-Abschnitte nur in Resedimenten von über $10 \mathrm{~cm}$ Mächtigkeit überliefert. Die weitaus überwiegende Zahl von Ereignislagen ist jedoch geringmächtiger und wird lediglich durch die oberen Y- und Z-Abschnitte der jeweils idealen Sequenz vertreten. Sie bilden also "base-cut-out"-Sequenzen (PIPER \& STOW 1991) der idealen Resediment-Abfolgen. Eine diesbezüglich gut untersuchte Ereignislage ist der Hauptturbidit (HT) des Eckfelder Grabungsprofils (vgl. Anlage 1). Im südöstlichen Grabungsareal erreicht er eine Mächtigkeit von $20 \mathrm{~cm}$ und besteht in seinem basalen Teil vorwiegend aus schlecht sortiertem, kiesgroßem Devonschutt (LUTZ 1993a, 1993e). In westlicher Richtung geht das extraklastenreiche Resediment über eine Distanz von nur $3 \mathrm{~m}$ bis $4 \mathrm{~m}$ in einen Bereich über, in dem Intraklasten als Sedimentkomponente dominieren. Begleitet ist der laterale Fazieswechsel von einer Mächtigkeitsabnahme auf rund $1 \mathrm{~cm}$ im äußersten NW der Grabungsfläche.

\section{Bestandteile der zentralen Seesedimente}

\subsection{Allochthone mineralische Sedimentanteile}

\subsubsection{Extraklasten}

Als Extraklasten werden hier die Fragmente von Gesteinen bezeichnet, die in ihrer stratigraphischen Position von den mitteleozänen Seeablagerungen abweichen bzw. außerhalb ihrer Grenzen entstanden sind. In den obersten 40m der Eckfelder Profundalsedimente können lithologisch vier Fremdgesteinsgruppen unterschieden werden.

Dunkle siltige Tonsteine (dunkelgrau bis graublau oder rötlichgrau): In eine hellglimmerreiche Tonmineral-Grundmasse sind eckige bis kantengerundete Quarzkörner regellos und matrixgestützt eingelagert. Die lithologische Variationsbreite reicht von schwach mittelsiltigen bis stark grobsiltigen Tonsteinen. Die Korngröße der Quarze ist dabei deutlich positiv mit inrer Häufigkeit korreliert. Fast reine Tonsteinklasten sind meist gut gerundet. Der Rundungsgrad nimmt jedoch mit steigendem Quarzanteil ab. 
Helle siltige Tonsteine (weiß bis gelblichweiß): Das Korngefüge dieser Klastengruppe zeigt starke Ähnlichkeit zu den dunklen Tonsteinen. Die Klasten sind jedoch entfestigt und weisen oft Anzeichen einer postsedimentären Deformation auf. In der Regel wurden härtere Gesteinsfragmente randlich in die hellen Tonsteine eingedrückt. Aufgrund ihrer geringen Härte ist die Form der Klasten sehr variabel.

Tonige Siltsandsteine (mittelgrau bis blaugrau oder rötlichgrau): Im Vergleich zu den siltigen Tonsteinen sind schlecht gerundete Quarzkörner sehr viel zahlreicher und mit größeren Durchmessern $(20 \mu \mathrm{m}$ bis $100 \mu \mathrm{m})$ im feinkörnigen Sediment vertreten. Das Korngefüge ist matrix-, häufig aber auch komponentengestützt. Aufgrund des gegenüber den Tonsteinen erhöhten Quarzanteils ist bei Siltsandsteinklasten häufig die Tendenz zu einer isometrischen Form zu beobachten.

Sandsteine bzw. quarzitische Sandsteine (hellgrau bis grünlichgrau oder gelblichgrau bis rotbraun): Monomineralische Sandsteine sind im Klasteninventar vergleichsweise unterreprä-

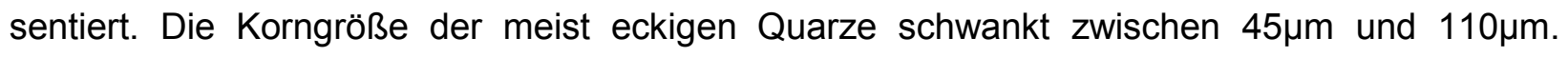
Größere Körner treten nur sehr untergeordnet auf. Meist sind die Quarze durch planare Kontaktflächen gegeneinander begrenzt, wenn auch gelegentlich Drucklösungserscheinungen, wie konkav-konvexe Korn/Kornkontakte, auftreten. Vereinzelt ist eine Zementierung des Korngefüges durch diagenetisch gebildete Quarzanwachssäume zu beobachten. Die Anwachssäume zeigen dabei generell die gleiche optische Orientierung wie die Quarzkörner, denen sie ansitzen. Sandsteinklasten sind in Abhängigkeit vom Zementationsgrad des Gesteins gerundet bis angerundet und häufig nahezu isometrisch.

Innerhalb der Lithofazieszonen 1: Minerogene Laminite und 2: Übergangsschichten bilden extraformationelle Siliziklasten neben detritischen Quarzkörnern einen Hauptbestandteil gradierter Sedimentabschnitte (Resedimente vom Typ-I bzw. Typ-II, vgl. Kap. 5.1.1 bzw. Kap. 5.1.2). Demgegenüber treten Extraklasten innerhalb der Lithofazieszone 3: Biogene Laminite lediglich an der Basis von Typ-III-Resedimenten massiert auf. Zum Hangenden hin werden sie zunehmend durch intraformationelle Laminit- und Phytoklasten abgelöst (vgl. Kap. 5.3). Abhängig von der jeweiligen Mächtigkeit eines gradierten Profilbereichs ( $>1 \mathrm{~m}$ bis $<1 \mathrm{~mm}$ ) schwankt die Klastengröße zwischen Stein und Feinsand.

Lithologisch lassen sich die nachgewiesenen Extraklasten den Gesteinen der UnteremsStufe (Unterdevon) zuordnen, die auch das tertiäre Eckfelder Maar unmittelbar umgeben (MEYER et al. 1994, FISCHER 1999). Im Raum Manderscheid/Eckfeld wird das Unterems durch eine Wechselfolge von vorwiegend grau, rötlich oder grünlich gefärbten Silt- und Tonsteinen sowie Sandsteinen und quarzitischen Sandsteinen vertreten (KöLSCHBACH et al. 1993, MEYER et al. 1994). Bei den extraformationellen Komponenten innerhalb der feinkörnigen Seeablagerungen handelt sich also um Bruchstücke der präeruptiven Gesteinssäule, die im Zuge der phreatomagmatischen Eruptionen im Bereich des heutigen Eckfelder Maares fragmentiert wurde. Die hellen entfestigten Tonsteinklasten sind als Reste einer mesozoisch-tertiären Verwitterungsdecke (MTV) anzusprechen, welche das unverwitterte Devon in unbekannter Mächtigkeit überlagerte (FISCHER 1999, FISCHER et al. 2000). Mafische Lapilli, die in der Bohrung E1/96 unterhalb von 44m Teufe häufig nachzuweisen sind (FISCHER 1999), haben innerhalb der hangenden Seesedimente keine Bedeutung. 


\subsubsection{Detritische Quarzkörner}

Detritische Quarzkörner treten innerhalb der beckenzentralen Seefazies in einem auffallend engen Korngrößenspektrum zwischen Grobsilt und sehr feinem Sand auf. Vorherrschend sind eckige bis kantengerundete Quarze mit Korngrößen von $20 \mu \mathrm{m}$ bis $80 \mu \mathrm{m}$ (Anhang 2-1, Fig. 02). Größere Quarzkörner mit einem mittleren Durchmesser bis maximal $120 \mu \mathrm{m}$ sind vergleichsweise selten.

In den rein mineralischen Sedimenten der Lithofazieszone 1 stellen isolierte oder in Lagen angereicherte Quarzkörner eine wesentliche Gesteinskomponente dar. Nach ZOLITSCHKA (1993) sind weite Teile der in der Bohrung von 1980 angetroffenen "bituminösen Laminite" im Hangenden der mineralischen Sedimente durch isoliert im Sediment verteilte Sandkörner charakterisiert, die vermutlich an den Wurzeln driftender Landpflanzen über die Seemitte transportiert wurden und anschließend als "tropical dropstones" auf die Sedimentoberfläche fielen. Tatsächlich ist das Auftreten von Quarzen in den Lithofazieszonen 2: Übergangsschichten und 3: Biogene Laminite jedoch weitgehend an klastenführende Lagen gebunden. Das in diesem Zusammenhang bei ZOLITSCHKA (1993) abgebildete Foto (Fig. 3, S. 482) zeigt eine Sedimentsequenz, die nach ihrem Interngefüge eindeutig einem Typ-III-Resediment zugeordnet werden kann. Neben dem gehäuften Auftreten von Quarzkörnern ist dieser Resediment-Typ durch hohe Gehalte an meist normalgradiert eingelagerten Laminit-Fragmenten charakterisiert (vgl. Kap. 5.1.3). Zwar ist nicht auszuschließen, daß gelegentlich Pflanzen aus den Uferregionen, beispielsweise durch anhaltende Wellentätigkeit, entwurzelt wurden und über den See drifteten - ein Vorgang, den auch GOTH (1990) als Quelle für gröberklastisches Material innerhalb der feinkörnigen Profundalsedimente von Messel für wahrscheinlich hält -, doch sprechen die Texturmerkmale quarzführender Profilabschnitte eher für einen vorherrschenden Eintrag von Quarzkörnern durch beckenwärts gerichtete bodennahe Dichteströme (siehe auch MINGRAM 1994).

In ihrer Größe und ihrem Rundungsgrad entsprechen die detritischen Quarze eindeutig der Quarz-Komponente in extraformationellen Klasten (vgl. Kap. 6.1.1). Als Liefergesteine können somit die Silt- und Sandsteine des fragmentierten Unterdevons gelten. Das auffallend enge Korngrößenspektrum detritischer Quarzkörner (Grobsilt bis Feinsand) innerhalb der Eckfelder Profundalsedimente ist also kein Ergebnis eines transportenergetischen Sortierungsprozesses, sondern vielmehr Ausdruck für die limitierte Verfügbarkeit von Quarzkorngrößen.

\subsubsection{Titan-Oxide}

In den rein mineralischen Sedimenten der Lithofazieszone 1: Minerogene Laminite wird die Siltfraktion neben häufigem Quarz von einem körnigen, zwischen $3 \mu \mathrm{m}$ und $7 \mu \mathrm{m}$ großen Mineral dominiert, daß nach EDX-Analysen hohe Gehalte an Titan und Sauerstoff aufweist (Anhang 21, Fig. 02). Im Dünnschliff (Durchlicht, Hellfeld) erscheinen die Mineralkörner durchsichtig farblos bis schwach gelblich und zeigen ein starkes, positives Relief gegenüber der umgebenden Tonmatrix sowie ein deutliches Chagrin. Die Interferenzfarbe ist bei einer Schliffdicke von $25 \mu \mathrm{m}$ ein blasses Gelb höherer Ordnung. Aufgrund ihrer geringen Größe ist der optische Charakter der Minerale nicht eindeutig bestimmbar, die Analyse einer Gesamtprobe mittels XRD wies jedoch Anatas, ein Titan-Oxid der Rutil-Gruppe $\left(\mathrm{TiO}_{2}\right)$, als Bestandteil der mineralischen Sedi- 
mente nach. Die lichtoptischen Eigenschaften und der oftmals körnige Habitus von Anatas (TRÖGER 1967) entsprechen den oben genannten Dünnschliffbeobachtungen.

Anatas entsteht infolge tropischer Verwitterung als ein häufiges Umwandlungsprodukt titanreicher Minerale, wie beispielsweise Ti-Augit, IImenit oder Titanit (TRÖGER 1967, PHILLIPS \& GRIFFEN 1981). Titanreicher Augit, kaersutitischer Pargasit und Titanit wurden als titanhaltige Mineralkomponenten bereits in der pyroklastischen Randfazies von Eckfeld (ZsÓTÉR 1997) bzW. im Hauptturbidit der Grabungsstelle (LUTZ 1993a) nachgewiesen.

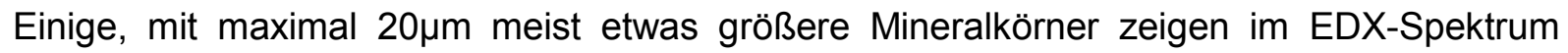
neben Titan und Sauerstoff auch Beimengungen von Silizium, Aluminium und in geringerem Maße von Eisen und Magnesium (Anhang 2-13, Fig. 01). Sie repräsentieren unvollständig verwitterte Mineralphasen primärer Ti-Silikate. Die unter dem Begriff "Leukoxen" zusammengefaßten, feinkörnigen Verwitterungsprodukte Ti-haltiger Minerale (TRÖGER 1967) konnten ebenfalls in den Seeablagerungen von Messel nachgewiesen werden (WEBER \& ZIMMERLE 1985).

\subsubsection{Tonminerale}

Zur Tonmineralogie der Eckfelder Tertiärsedimente liegt bereits eine ganze Reihe von Untersuchungen vor, die im Folgenden kurz zusammengefaßt werden sollen.

In den Profilbereichen unmittelbar im Liegenden der Lithofazieszone 1: Minerogene Laminite bilden Chlorit (Klinochlor), Illit/Muskovit, Kaolinit und Quarz den pelitischen Matrixanteil grobklastischer Resedimente. Auffallend helle Matrixbereiche führen dagegen Smectit auf Kosten von Chlorit und können nach FISCHER (1999) aus den Resten der eruptiv fragmentierten mesozoisch-tertiären Verwitterungsdecke abgeleitet werden. Klinochlor ist nach eigenen XRDAnalysen ebenfalls ein häufiges Matrixmineral der Mikrofazieszone 1a: Quarz-Fazies.

NEGENDANK et al. (1982) führten umfangreiche Tonmineralanalysen am Bohrprofil von 1980 durch (vgl. Kap. 2.3). Innerhalb der Lithofazieszone 3: Biogene Laminite ("Seesedimente der 1. Phase" und "Übergangszone", sensu NEGENDANK et al. 1982) dominieren Montmorillonit, "kaum illitischer Glimmer" bzw. "Mica" (sensu NEGENDANK et al. 1982) und Kaolinit das Tonmineralspektrum. Chlorit tritt nur an der Basis des von NEGENDANK et al. (1982) untersuchten Profilabschnitts bei Bohrmeter 49,5 auf.

Im Bereich des Eckfelder Grabungsprofils konnten Illit, Smectit und Kaolinit als häufige Tonmineralkomponente sowohl in den laminierten Sedimentabschnitten, als auch in den feinkörnigen Toplagen von Turbiditen (Z-Abschnitte, vgl. Kap. 5.1.3) nachgewiesen werden (LUTZ 1993a, MINGRAM 1994, 1998). ZsóTÉR (1997) untersuchte die Lapilli- bzw. Lapilli-Aschen-Tuffe am Süd- und Nordrand ("Ostschurf") des ehemaligen Maarkraters. Unter den Matrixmineralen der Tuffe dominieren Kaolinit und in untergeordnetem Maße Smectit (Montmorillonit).

Chlorit scheint in seinem Auftreten vorwiegend an die mineralischen Sedimente im Liegenden der Lithofazieszone 3: Biogene Laminite gebunden zu sein. Zusammen mit Muskovit und Sericit ist er ein Bestandteil der devonischen Pelite in der unmittelbaren Umgebung des Eckfelder Maares (FISCHER 1999). Unter Erhaltung des strukturellen Gerüstes kann primärer Muskovit zu dem kaliumärmeren Illit degradieren. Smectit (Montmorillonit) und Kaolinit können ebenfalls ein Produkt der Glimmerverwitterung sein (KöSTER 1974, HEIM 1990, JASMUND 1993). Beide 
Tonminerale entstehen aber auch bei der Alternation basaltischer Pyroklastika und vulkanischer Aschen unter tropischen und subtropischen Bedingungen (HEIM 1990, ZSÓTÉR 1997). In tropischen Klimaten ist Kaolinit daher häufig mit Anatas assoziiert (SCHWERTMANN \& NiEDERBUDDE 1993), der als Verwitterungsprodukt ebenfalls in den Sedimenten der Lithofazieszone 1: Minerogene Laminite nachgewiesen wurde. Kaolinit bildet weiterhin ein stabiles Endprodukt bei der Zersetzung feldspatführender Silikatgesteine (HEIM 1990, JASMUND 1993). Das sporadische Auftreten von Feldspat als untergeordnetes Detritusmineral ist aus der gesamten Abfolge der Eckfelder Maarsedimente belegt (NEGENDANK et al. 1982, LUTZ 1993a, MINGRAM 1998, FISCHER 1999).

\subsection{Allochthone organische Sedimentanteile}

\subsubsection{Ulminit}

Tab. 14: Position von Ulminit innerhalb der Mazeralklassifikation

\begin{tabular}{|c|c|c|c|}
\hline Mazeral-Gruppe & Mazeral-Subgruppe & Mazeral & Mazeral-Typ \\
\hline Huminit & Humotelinit & Ulminit & $\begin{array}{c}\text { Texto-Ulminit } \\
\text { Eu-Ulminit }\end{array}$ \\
\hline
\end{tabular}

Unter der Bezeichnung Ulminit werden in Vergelung begriffene oder bereits komplett vergelte pflanzliche Zellwände von isoliert auftretenden Einzelzellen und von Zellgeweben zusammengefaßt (ICCP 1971).

Das Grundgerüst makrophytischer Zellwände besteht vorwiegend aus Zellulose. In verholzten Geweben sind zusätzlich wechselnde Gehalte von Lignin fein verteilt zwischen den Zellulose-Mikrofibrillen inkrustiert (TAYLOR et al. 1998). Die Zersetzung pflanzlicher Zellwände ist hauptsächlich auf eine Biodegradation des Zellulose-Gerüstes zurückzuführen. STOUT \& SPACKMAN (1987) beobachteten an Torf-Proben aus dem südlichen Florida und dem Okefenokee-Sumpfgebiet (Georgia, USA) die punktuelle Abnahme der optischen Anisotropie von Holzgewebe als Folge eines selektiven Zellulose-Abbaus, der schließlich zu einem Verlust der strukturellen Integrität degradierter Zellwände führt. Lignin ist generell widerstandsfähiger gegen biochemische Zersetzung als Zellulose (STOUT et al. 1981, HARMON et al. 1986, BUSTIN et al. 1985, TeICHMÜLleR 1989, Diessel 1992, TAYLOR et al. 1998, ScOtT 2002). Das Vorkommen und die chemische Zusammensetzung von Lignin-Polymeren kann jedoch zwischen verschiedenen Gewebearten derselben Pflanze - beispielsweise zwischen Holz- und Rindengewebe - aber auch innerhalb des selben Gewebes zwischen der primären und der sekundären Zellwand stark variieren (HEDGES et al. 1985, ERIKSSON et al. 1990, TAYLOR et al. 1998). Vergleichsweise schwer zersetzbares Guajacyl-Lignin ist typisch für Gymnospermen-Hölzer, während Syringyl-Lignin neben anderen Lignin-Varietäten vorwiegend in Angiospermen auftritt (RUSSELL \& BARRON 1984, HATCHER et al. 1989 a, b).

Die Bildung von Huminsäuren aus der biochemischen Degradation hauptsächlich von Lignin und Zellulose wird als Humifizierung bezeichnet (TAYLOR et al. 1998). Auch wenn der Prozeß der Humifizierung noch nicht in allen Einzelheiten verstanden wird (TYSON 1995), so gilt doch als sicher, daß die Depolymerisierung des Lignin-Komplexes weitgehend aerob unter der Beteiligung von Pilz-Enzymen erfolgt (TEICHMÜLLER \& TEICHMÜLLER 1982, CHAFFEE et al. 1984). Rezent sind Pilze die bedeutendsten Destruenten von Holzgewebe in terrestrischen Öko- 
systemen (ERIKSSON et al. 1990) und zeigen ihre größte Aktivität scheinbar generell in der humusreichen Streuschicht ("surface litter") des Bodens (STOUT et al. 1989). Bakterien spielen bei der Degradation pflanzlichen Materials eine vergleichsweise untergeordnete Rolle (RAYNER \& BODDY 1988), gewinnen aber unter wassergesättigten, sauerstofflimitierten Bedingungen an Bedeutung (EATON \& HALE 1993). Neben der Verfügbarkeit von Sauerstoff fördert die Zufuhr von Nährstoffen, ein pH-Wert im schwach alkalischen bis schwach sauren Bereich sowie eine vergleichsweise hohe Temperatur am Ablagerungsort die Aktivität von Destruenten und somit die Humifizierung pflanzlicher Gewebe (DIESSEL 1992, TAYLOR et al. 1998). Dabei gehen nicht nur die Stoffwechselprodukte von Pilzen und Bakterien in den Humifizierungsprozeß ein, sondern teilweise auch die Biomasse der Reduzenten als solche (COHEN et al. 1987, TEICHMÜLLER 1989, TAYLOR et al. 1998). Es entsteht ein weites Spektrum colloidaler huminitischer Substanzen bestehend aus einer komplexen Mischung von Carbon-, Phenol- und anderen organischen Säuren (DIESSEL 1992). Mit progressiver Diagenese setzt der Prozeß der biochemischen Vergelung ein. Nach ICCP (1971) und TAYLOR et al. (1998) geht die Gelbildung auf eine fortschreitende Peptisierung und Ausfällung von Humuskolloiden zurück. Biochemische Vergelung tritt häufig in subaquatischen Milieus auf und scheint durch anoxische Bedingungen gefördert zu werden (ICCP 1971, TEICHMÜLLER 1989, DIESSEL 1992, TAYLOR et al. 1998).

Im Falle der Ulminit-Bildung findet eine in-situ-Vergelung pflanzlicher Gewebe statt, die anfänglich zu einer Verdickung der Zellwände und schließlich zu ihrer vollständigen Homogenisierung führt. Es handelt sich also um eine pseudomorphologische Transformation ligno-zelluloser Zellwände in amorphe Humusgele (TAYLOR et al. 1998). Nach dem Grad der Vergelung werden die Mazeral-Typen Texto-Ulminit und Eu-Ulminit unterschieden.

\section{Texto-UIminit}

Der Mazeral-Typ Texto-Ulminit ist die unvollständig vergelte Form des Ulminits. Bei schwach vergelten Texto-Ulminiten innerhalb der Eckfelder Seesedimente ist das ursprüngliche Zellgefüge immer noch deutlich zu erkennen (Anhang 2-9, Fig. 01). In seltenen Fällen blieben auch interzellulare Hohlräume zwischen den Zellwänden erhalten. Eine sichere Zuordnung zu diskreten pflanzlichen Gewebetypen ist jedoch durch die allgemein starke Kompaktion der Gewebereste kaum möglich.

Bei intensiv vergelten Texto-Ulminiten sind die geschwollenen und homogenisierten Zellwände in der Regel sehr viel dicker als der Durchmesser der erhalten gebliebenen Zell-Lumen. In solchen Fällen ist die ehemalige Gewebestruktur durch eine undeutliche Aneinanderreihung unregelmäßiger Hohlräume zu erahnen (Anhang 2-9, Fig. 02).

Unter Auflicht reflektieren Texto-Ulminite schwach bis moderat, zeigen jedoch, von wenigen Ausnahmen abgesehen, eine allgemein höhere Reflexionsintensität als benachbarte Humodetrinite und Corpohuminite. Die unterschiedliche Stärke in der Reflexion vergelter Pflanzengewebe ist wahrscheinlich auf variierende Beimengungen von Harz, Cutin, Tannin oder anderer Zellwand imprägnierender Substanzen zurückzuführen (TAYLOR et al. 1998).

\section{Eu-Ulminit}

Im Unterschied zu Texto-Ulminit ist Eu-Ulminit durch das Fehlen geöffneter Zell-Lumen gekennzeichnet. Nach DIESSEL (1992) entstehen Eu-Ulminite zum einen syngenetisch durch 
das vollständige Kollabieren von Zellgewebe während des Humifizierungsprozesses, zum anderen epigenetisch durch die Imprägnierung von beispielsweise Texto-Ulminit durch fluide Humuskolloide. Eine in den Eckfelder Sedimenten mehrfach beobachtete, undeutliche und unterbrochene Streifung von Eu-Ulminiten ist sicherlich dem ersten Entstehungsprozeß zuzuordnen. Weitaus häufiger tritt der Mazeral-Typ jedoch als massive Gelkörper ohne signifikante Mikrostruktur auf, die in randlichen Bereichen jedoch häufig Übergänge zum schwächer vergelten Texto-Ulminit zeigen (Anhang 2-9, Fig. 03).

Das Vorkommen von Ulminiten ist mit einer durchschnittlichen Häufigkeit von 0,4 Vol.\% (Texto-Ulminit) bzw. 0,2 Vol.\% (Eu-Ulminit) vorwiegend an die tonreichen Laminen der Lithofazieszonen 2: Übergangsschichten und 3: Biogene Laminite gebunden. Mit einer Länge, die selten $150 \mu \mathrm{m}$ übersteigt, sind sie allgemein mit weiteren detritischen Huminit-Partikeln, wie Humodetrinit, Detro-Gelinit und Corpohuminit, assoziiert. Als Phytoklasten bilden Ulminite weiterhin einen häufigen Bestandteil von Resedimenten des Typs II bzw. III. Ihre Länge variiert hier in Abhängigkeit von der Gesamtmächtigkeit einer gradierten Schicht zwischen mehreren Zentimetern und weniger als $100 \mu \mathrm{m}$.

\subsubsection{Humodetrinit}

Tab. 15: Position von Humodetrinit innerhalb der Mazeralklassifikation

\begin{tabular}{cccc}
\hline Mazeral-Gruppe & Mazeral-Subgruppe & Mazeral & Mazeral-Typ \\
\hline Huminit & Humodetrinit & \\
\hline
\end{tabular}

Als Humodetrinit werden huminitische Pflanzengewebe-Bruchstücke von subzellularer Größe (meist $<10 \mu \mathrm{m}$ ) und variabler Form bezeichnet. In Torfen und Braunkohlen bildet Humodetrinit zusammen mit fein verteilten, unfigurierten Humusgelen häufig eine Art Grundmasse, die je nach dem Grad der Vergelung in die Mazerale Attrinit oder Densinit unterteilt wird (ICCP 1971, TEICHMÜLLER 1989, TAYLOR et al. 1998). Innerhalb der Eckfelder Profundalsedimente treten Zell-Bruchstücke und weitere Kleinstreste pflanzlicher Gewebe zwar häufig aber meist isoliert auf (Anhang 2-9, Fig. 04), so daß die Mazeralbestimmung in diesem Falle auf der SubgruppenEbene verbleibt.

Mit durchschnittlich 8,0 Vol.\% (maximal 41,1 Vol.\% in Probe KS9) dominieren Humodetrinite klar das Spektrum huminitischer Partikel innerhalb der organisch-reichen Lithofazieszonen 2 und 3. In der Vorherrschaft von huminitischem Feinstdetritus gegenüber größeren Geweberesten (Texto- und Eu-Ulminit) deutet sich der intensive biochemische Abbau pflanzlichen Materials unter warm-humiden Bedingungen an. Der auffallend hohe Destruktionsgrad pflanzlicher Gewebereste steht auch im Einklang mit den aus paläobotanischen Befunden abgeleiteten Vorstellungen über die Art der Vegetation im unmittelbaren Randbereich des EckfeldSees. Nach pollenanalytischen Untersuchungen von NICKEL (1996) war das Gewässer von einer Juglandaceen-dominierten Waldvegetation umstanden. Die Omnipräsenz von Angiospermen (hauptsächlich von Dicotyledoneae) äußert sich ebenfalls im Spektrum der in Eckfeld geborgenen botanischen Makroreste (FRANKENHÄUSER \& WILDE 1993, LUTZ et al. 1998, WILDE \& FRANKENHÄUSER 1998). Neben den zellulosereichen (ligninarmen) Geweben krautiger 
Pflanzen sind hauptsächlich die Hölzer von Angiospermen als Mazeralvorläufer des Humodetrinits anzusehen (TEICHMÜLLER 1989, siehe auch V. D. BRELIE \& WOLF 1981). In Relation zu Gymnospermen-Hölzern weisen die Zellgewebe von Angiospermen ein prinzipiell geringeres Erhaltungspotential auf (RUSSELL \& BARRON 1984, HEDGES et al. 1985, DIESSEL 1992, TAYLOR et al. 1998). Dieser Umstand ist zum Teil auf die geringeren Gehalte an Lignin in Angiospermen-Geweben zurückzuführen (HARMON et al. 1986, ERIKSSON et al. 1990), aber auch auf die höhere Anfälligkeit von Angiospermen-Lignin gegenüber biochemischer Degradation (RAYNER \& BODDY 1988). Generelle Unterschiede in der Holzanatomie beider PflanzenUnterabteilungen spielen ebenfalls eine Rolle. Die Gefäße von Angiospermen sind meist größer dimensioniert als Gymnospermen-Tracheiden und ermöglichen so ein schnelleres Eindringen von Pilzen (HARMON et al. 1986).

Ähnlich wie im tertiären Hauptflöz der rheinischen Braunkohle (V. D. BRELIE \& WOLF 1981) und den eozänen Braunkohlen von Helmstedt (RIEGEL et al. 1999, 2002) ist also auch im Falle der Eckfelder Seeablagerungen davon auszugehen, daß die auffallend hohen Gehalte an Humodetrinit in der Hauptsache durch den Eintrag stark degradierter Gewebe einer angiospermenbetonten Vegetation bedingt sind.

\subsubsection{Gelinit}

\begin{tabular}{cccc}
\hline Mazeral-Gruppe & Mazeral-Subgruppe & Mazeral & Mazeral-Typ \\
\hline Huminit & Humocollinit & Gelinit & $\begin{array}{c}\text { Levigelinit } \\
\text { (Detro-Gelinit, Eu-Gelinit) }\end{array}$ \\
\hline
\end{tabular}

Die in den Eckfelder Sedimenten auftretenden Gelinite sind unfigurierte Hohlraumfüllungen ausgefällter Humusgele oder vollständig vergelte Kleinst-Bruchstücke pflanzlicher Gewebe. Unter den Geliniten wird der feinporöse bis mikrokörnige Mazeral-Typ Pori-Gelinit gegen den strukturlosen Typ Levigelinit abgegrenzt. Poröse Gelinite sind in Eckfeld lediglich als ZellLumenfüllungen vertreten, die optisch von primären Zellexkreten nicht zu unterscheiden sind (Anhang 2-9, Fig. 05). Sie wurden daher definitionsgemäß (ICCP 1971) zu den Corpohuminiten (vgl. Kap. 6.2.4) gestellt. Levigelinit kommt in den Varietäten Detro-Gelinit und Eu-Gelinit vor.

\section{Detro-Gelinit}

Als Detro-Gelinit wurden strukturlose, nahezu homogene Gel-Partikel mit einem Durch-

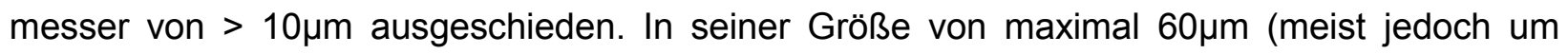
$25 \mu \mathrm{m}$ ) ist der Detro-Gelinit dem Mazeral Corpohuminit ähnlich, zeichnet sich im Gegensatz dazu jedoch durch einen stark variablen Umriß aus (Anhang 2-9, Fig. 06). Detro-Gelinite sind das Ergebnis einer intensiven Vergelung von huminitischem Feindetritus und stehen damit genetisch den Humodetriniten nahe (ICCP 1971, TEICHMÜLLER 1989, DIESSEL 1992, TAYLOR et al. 1998). Gelegentlich ist auch noch die ehemalige Form einzelner Detritus-Partikel innerhalb des Gelkörpers nachvollziehbar (Anhang 2-9, Fig. 07).

Detro-Gelinite sind mit durchschnittlich 0,6 Vol.\% an der Zusammensetzung der zentralen Seesedimente beteiligt und kommen hier isoliert vorwiegend in tonreichen Laminen der Lithofazieszonen 2 und 3 vor. 


\section{Eu-Gelinit}

Eu-Gelinite sind massive Gelkörper, die unter Auflicht keine mikrostrukturellen Hinweise auf vergelte Pflanzengewebe liefern. Auch der meist leistenförmige Habitus deutet eher auf die Humusgelfüllung länglicher Hohlräume, wie beispielsweise Schrumpfungsrisse oder Wurzelbahnen, hin. Die Länge der in Eckfeld nachgewiesenen Eu-Gelinite übersteigt jedoch selten $200 \mu \mathrm{m}$. Gelgefüllte Risse oder Sedimentfugen sind in den feinkörnigen Ablagerungen der zentralen Seefazies in situ nicht beobachtet worden. Die generelle Einregelung der länglichen GelPartikel parallel zur Schichtung sowie ihre Bindung an humotelinit- und humodetrinitreiche Tonlaminen weisen eher auf eine allochthone Herkunft der Eu-Gelinite hin. Mit durchschnittlich 0,1 Vol.\% hat der Mazeral-Typ jedoch nur einen sehr geringen Anteil an der Sedimentzusammensetzung.

\subsubsection{Corpohuminit}

Tab. 16: Position von Corpohuminit innerhalb der Mazeralklassifikation

\begin{tabular}{|c|c|c|c|}
\hline Mazeral-Gruppe & Mazeral-Subgruppe & Mazeral & Mazeral-Typ \\
\hline Huminit & Humocollinit & Corpohuminit & $\begin{array}{l}\text { Corpohuminit (isoliert) } \\
\text { Corpohuminit (in situ) }\end{array}$ \\
\hline
\end{tabular}

Corpohuminite sind primäre oder sekundäre Zell-Lumenfüllungen, die sich zum einen aus phlobaphenitischen Zellexkreten der noch lebenden Pflanze, zum anderen aus der postmortalen Infiltration von Zellkörpern durch kolloidale Humuslösungen herleiten lassen (ICCP 1971). Primäre Zellexkrete stammen vorwiegend von den Oxidations- oder Kondensationsprodukten des Tannins ab und treten häufig in den abgestorbenen Zellen des Rindengewebes, besonders in Korkzellen auf (ICCP 1971, TAYLOR et al. 1998, DIESSEL 1992). Gestalt und Größe der Corpohuminite entsprechen naturgemäß den Dimensionen pflanzlicher Zellen. Bei der Erfassung des organischen Detritus innerhalb der Eckfelder Schwarzpelite wurden isoliert auftretende von in situ (d.h. im Gewebeverband) vorkommenden Corpohuminiten unterschieden.

\section{Corpohuminit (isoliert)}

Isolierte Corpohuminite sind meist homogene, gelegentlich kavernöse Gelkörper von nahezu runder bis ovaler Form. Ihre Durchmesser schwanken üblicherweise zwischen $20 \mu \mathrm{m}$ und $50 \mu \mathrm{m}$ (Anhang 2-9, Fig. 08). Selten wurden langgestreckt eckige Zellfüllungen mit einer Länge von maximal $200 \mu \mathrm{m}$ beobachtet. Mit durchschnittlich $0,5 \mathrm{Vol} \%$ sind isolierte Corpohuminite ein untergeordneter aber regelmäßiger Bestandteil der Eckfelder Profundalsedimente. Sie dominieren mit einem Verhältnis von fast 10:1 eindeutig über in situ auftretende Zell-Lumenfüllungen.

\section{Corpohuminit (in situ)}

Dieser Mazeral-Typ kommt vorwiegend als Zell-Lumenfüllung in Eu-Ulminiten, seltener auch in Texto-Ulminiten vor. Aufgrund ihrer chemischen und strukturellen Stabilität sind Corpohuminit-Ansammlungen jedoch vielfach auch die einzigen erhalten gebliebenen Relikte ehemaliger Zellgewebe, während die weniger beständigen Zellwände im mikroskopischen Bild oft nicht mehr nachzuweisen sind (Anhang 2-10, Fig. 01). Gelegentlich wurden feinporöse Zell- 
füllungen (Pori-Gelinit) in Assoziation mit schwach vergelten Texto-Ulminiten beobachtet, deren Zellwände teilweise intern separiert sind bzw. vollständig degradiert wurden (Anhang 2-9, Fig. 05). Nach LIU et al. (1982) und DIESSEL (1992) beginnt die Degradation von Zellwänden meist mit ihrer Aufspaltung in feine Lamellen, gefolgt von einer Transformation in tropfenförmige Humuskolloide. Diese schlagen sich anschließend als Vorläufer des Pori-Gelinits in angrenzenden Zellhohlräumen nieder. STOUT \& SPACKMAN (1987) beobachteten an Gefäß-Elementen von Rhizophora aus den subrezenten Torfen der südlichen USA eine selektive Degradation der sekundären Zellwand und die Akkumulation eines granularen Rückstandes in angrenzenden Zell-Lumen.

Eine Zuordnung von in situ auftretenden Corpohuminiten zu bestimmten pflanzlichen Gewebetypen gelingt nur in Ausnahmefällen. Mehrfach nachgewiesene stapelförmige Ansammlungen von plattigen rund $20 \mu \mathrm{m}$ langen Zellfüllungen sind jedoch von Korkgewebe herzuleiten (Anhang 2-10, Fig. 01).

\subsubsection{Sporinit}

Tab. 17: Position von Sporinit innerhalb der Mazeralklassifikation

\begin{tabular}{clll}
\hline Mazeral-Gruppe & Mazeral-Subgruppe & Mazeral & Mazeral-Typ \\
\hline Liptinit & Sporinit & \\
\hline
\end{tabular}

Sporinite sind die äußeren Zellwände (Exinen und Perinen) von Sporen und Pollenkörnern. Sie bestehen aus Sporopollenin, einer nach SHAW (1971) und TEICHMÜLLER (1989) resistenten, hoch polymerisierten und quervernetzten Substanz aus Carotinoiden und/oder CarotinoidEstern. Neuere Analysen zeigen jedoch, daß wenigstens zwei Typen von Sporopollenin mit aromatischen oder aliphatischen Bausteinen chemisch unterschieden werden können (SCOTT 2002). Während der Diagenese gehen die aromatischen Bestandteile allem Anschein nach zugunsten der aliphatischen Komponente selektiv verloren. Das fossile Äquivalent zum Sporopollenin rezenter Pollen und Sporen wird als Sporonin bezeichnet (TAYLOR et al. 1998).

Die Sporinite der Eckfelder Profundalsedimente sind schichtparallel eingeregelt und üblicherweise stark kompaktiert. In den meisten Fällen ist der ursprüngliche Hohlraum jedoch erhalten geblieben und als schmale Linie zwischen den kräftig grüngelb fluoreszierenden Zellwänden zu erkennen (Anhang 2-10, Fig. 02 und Fig. 03). Mit einer üblichen Länge von 10 $\mu \mathrm{m}$ bis $50 \mu \mathrm{m}$, meist jedoch im Bereich zwischen $20 \mu \mathrm{m}$ und $30 \mu \mathrm{m}$, bilden Sporinite einen regelmäßigen Bestandteil der Lithofazieszonen 2: Übergangsschichten und 3: Biogene Laminite. Abgesehen von einem leichten Maximum von 1,3 Vol.\% in Probe KS23 (MFZ 3d: AulacoseiraOpal(CT)-Fazies) ist ihre vertikale Häufigkeitsverteilung mit Werten zwischen 0,1 Vol.\% und 0,9 Vol.\% nur geringen Schwankungen unterworfen. Lediglich in den rein mineralischen Sedimenten der Lithofazieszone 1: Minerogene Laminite (Probe KS1) und in einer Probe aus der MFZ 3b: Aulacoseira-Fazies (KS13) konnten keine Sporinite nachgewiesen werden. Eine Zuordnung von Sporinit zu bestimmten Sporomorphen-Taxa gelingt in Gesteinsschliffen meist nicht (TEICHMÜLLER 1989). Auf Sedimentanbrüchen parallel zur Schichtung ist eine fluoreszenzmikroskopische Identifizierung mit Einschränkungen jedoch möglich (Anhang 2-10, Fig. 04). Nach palynologischen Untersuchungen von NICKEL (1994, 1996 und frndl. schriftl. Mitt. 
2001) dominieren die Pollen von Angiospermen, vornehmlich von Walnußgewächsen (Juglandaceen) und Buchengewächsen (Fagaceen) bei weitem das Sporomorphen-Spektrum in Eckfeld.

\subsubsection{Cutinit}

Tab. 18: Position von Cutinit innerhalb der Mazeralklassifikation

\begin{tabular}{clll}
\hline Mazeral-Gruppe & Mazeral-Subgruppe & Mazeral & Mazeral-Typ \\
\hline Liptinit & Cutinit \\
\hline
\end{tabular}

Cutinit ist die Mazeralbezeichnung für die Überreste von wasserabstoßenden Kutikeln, die an der Oberfläche von Blättern, Zweigen und anderen der Atmosphäre ausgesetzten Pflanzenteilen gebildet werden (TEICHMÜLLER 1989, TAYLOR et al. 1998). Rezente Kutikeln bestehen im wesentlichen aus Cutin, das als Verdunstungsschutz von den Zellulose-Wänden der Epidermis nach außen hin abgeschieden wird. Im chemischen Sinne ist Cutin ein aus Hydroxy- und Epoxy-Fettsäuren bestehender Biopolyester (DIESSEL 1992, TAYLOR et al. 1998). Durch den hochpolymeren Charakter des Cutins sind Cutinite weitgehend resistent gegenüber mikrobieller Zersetzung und atmosphärischer Oxidation (COOK \& KANTSLER 1982, TAYLOR et al. 1998). In Schnitten senkrecht zur Schichtung zeigen Cutinite typischer Weise die Form von einfachen oder einseitig gezähnten Bändern. Im Horizontalschnitt ist gelegentlich noch das Prägemuster der darunter liegenden Epidermis-Zellen sichtbar.

Innerhalb der beckenzentralen Fazies von Eckfeld sind Cutinite mit durchschnittlich 0,1 Vol.\% an der Summe der Sedimentbestandteile beteiligt und zeigen nur wenige, sporadisch auftretende Häufigkeitsmaxima bis $0,5 \mathrm{Vol}$ \% Sie treten mit einer Länge von wenigen $100 \mu \mathrm{m}$ gelegentlich zusammen mit kleineren Huminiten, wie Humodetrinit, Corpohuminit und DetroGelinit, in dunklen, organisch-reichen Laminen auf. Oft gut erhaltene, mehrere Millimeter lange Cutinite sind dagegen neben größeren Ulminiten häufiger Bestandteil von Typ-III-Resedimenten (vgl. Kap. 5.1.3). Die in Eckfeld nachgewiesenen Blattkutikeln lassen sich grundsätzlich zwei Morphotypen zuordnen:

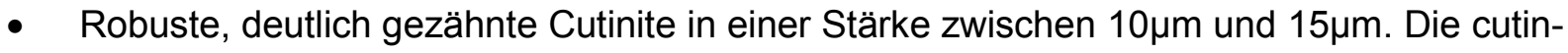
reiche Außenschicht, die Cuticula im eigentlichen Sinne, zeigt eine meist kräftig gelbe Fluoreszenz. Eine oft beobachtete allmähliche Abnahme der Fluoreszenzintensität in Richtung Epidermis ist auf kontinuierlich sinkende Cutin- bzw. steigende Zellulosegehalte zurückzuführen. Die Substanz dieser Übergangsschicht wird als Cutozellulose bezeichnet und führt neben Cutin und Zellulose auch Anteile von Wachs und Pektin (ICCP 1971, TAYLOR et al. 1998). Bei einigen nachgewiesenen Cutiniten sind auch die cutinisierten Zellwände der Epidermis deutlich sichtbar erhalten geblieben (Anhang 2-10, Fig. 05).

- Dünne $(<5 \mu \mathrm{m})$ nur schwach oder ungezähnte Cutinite ohne erkennbare CutozelluloseSchicht. Die Cutinite treten meist in Form von isolierten, stark gefalteten Häuten von grünlicher oder gelblicher Fluoreszenz auf (Anhang 2-10, Fig. 06). 


\subsubsection{Resinit}

Tab. 19: Position von Resinit innerhalb der Mazeralklassifikation

\begin{tabular}{|c|c|c|c|}
\hline Mazeral-Gruppe & Mazeral-Subgruppe & Mazeral & Mazeral-Typ \\
\hline Liptinit & & Resinit & \\
\hline
\end{tabular}

Resinite entstehen aus einer Vielzahl pflanzlicher Zellexkrete, hauptsächlich jedoch aus den Harzen und Wachsen von Gefäßpflanzen (DIESSEL 1992). TEICHMÜLLER (1989) und TAYLOR et al. (1998) unterscheiden zwischen "Terpen-Resiniten" und "Lipid-Resiniten". In die erste Gruppe fallen die aus terpenartigen Vorläufersubstanzen gebildeten Resinite, die zusammenfassend als Harze bezeichnet werden. Harze entstehen in pflanzlichen Sekretzellen und sind fossil oft als Zell-Lumenfüllungen überliefert. "Lipid-Resinite" umfassen die aus Wachsen und Fetten ableitbaren Resinite. Teilweise sind auch ätherische Öle an der Resinitbildung beteiligt. Wachse werden hauptsächlich als korn- oder stabförmige Ausscheidungen bzw. als kompakte Harzkrusten vorwiegend auf Blättern und Früchten abgelagert (TEICHMÜLLER 1989). Im Gegensatz zu "Terpen-Resiniten" zeigen die von Lipiden abstammenden Resinite in der Regel nicht die Form pflanzlicher Zell-Lumen (TAYLOR et al. 1998).

Mit durchschnittlich 0,03 Vol.\% sind Resinite nur unwesentlich an der Zusammensetzung der partikulären organischen Substanz innerhalb der Eckfelder Beckensedimente beteiligt. Sie treten nahezu ausschließlich in situ als Zell-Lumenfüllungen von Ulminiten auf und sind demnach als "Terpen-Resinite" anzusprechen (Anhang 2-11, Fig. 01 und Fig. 02). Unter Auflicht erscheinen sie schwarz bis milchig grau und zeigen gelegentlich rotbraune Innenreflexe. Die Fluoreszenz ist grünlichgelb bis gelb. Von Wachsen ableitbare Resinite wurden lediglich in einem Fall nachgewiesen (Anhang 2-10, Fig. 05). Die kräftig gelb fluoreszierenden, ovalen bis spindelförmigen Resinit-Körner sitzen hier direkt der Cutin-Lage einer in Falten gelegten Blattkutikel auf und sind teilweise auch auf der der Epidermis zugewandten Seite zwischen zwei Cutozellulose-Schichten eingelagert (vgl. Kap. 6.2.6). Nach TAYLOR et al. (1998) beinhalten die cutinisierten Zellwände insbesondere von tropischen Pflanzen oftmals Wachse als Schutz gegen Austrocknung.

\subsubsection{Liptodetrinit}

Tab. 20: Position von Liptodetrinit innerhalb der Mazeralklassifikation

\begin{tabular}{|c|c|c|c|}
\hline Mazeral-Gruppe & Mazeral-Subgruppe & Mazeral & Mazeral-Typ \\
\hline Liptinit & & Liptodetrinit & \\
\hline
\end{tabular}

Liptodetrinit ist ein Sammelbegriff für Bestandteile der Liptinitgruppe, die aufgrund ihrer feindetritischen Beschaffenheit und/oder geringen Teilchengröße nicht mehr sicher einem der anderen Liptinitmazerale zugeordnet werden können (ICCP 1971, TAYLOR et al. 1998). Ein Teil des Liptodetrinits entsteht durch die mechanische Zerkleinerung oder chemisch-mikrobiologische Zersetzung von Sporen, Pollen, Kutikulen, Harzen, Wachsen und kutinisierten oder suberinisierten Zellwänden (ICCP 1971, COOK \& KANTSLER 1982). Insbesondere in Ölschiefern ist jedoch eine Vielzahl von Liptodetriniten vermutlich von Algen abzuleiten (SHERWOOD 1984). 
Innerhalb der Eckfelder Seesedimente zeigen Liptodetrinite gemäß ihres weiten Spektrums an Ausgangsmazeralen eine hoch variable Form. Die generell < 10 $\mathrm{m}$ großen Partikel sind jedoch immer durch eine kräftige bis moderate gelbgrüne Fluoreszenz gekennzeichnet. In denjenigen Proben, in denen der "kleine Kolonie-Typ" von Botryococcus (NICKEL 1994, 1996; vgl. Kap. 6.4.1) massenhaft auftritt ist mit abnehmender Koloniegröße ein fließender Übergang hin zu unregelmäßig körnigen Liptiniten zu beobachten, die aufgrund ihres geringen Durchmessers nicht mehr eindeutig als Botryococcus klassifiziert werden können (Anhang 2-10, Fig. 07). Sie wurden definitionsgemäß den Liptodetriniten zugeordnet.

Mit durchschnittlich 0,7 Vol.\% ist Liptodetrinit nach Botryococcus und Tetraedron (vgl. Kap 6.4.1) das dritthäufigste Liptinitmazeral innerhalb der Eckfelder Profundalsedimente. Nach COOK \& KANTSLER (1982) weisen Ablagerungen, die reich sind an strukturierten Liptiniten naturgemäß auch hohe Gehalte für Liptodetrinit auf. Dieses läßt sich im Falle von Eckfeld auch innerhalb der Bohrungen E1/96 bzw. E2/96 nachvollziehen. Insbesondere in der Lithofazieszone 3: Biogene Laminite ist die vertikale Häufigkeitsverteilung von Liptodetrinit tendenziell dem weitgehend durch die Abundanzen von Humodetrinit und Alginit geprägten Huminit:LiptinitVerhältnis gegenläufig (Anlage 2).

\subsection{Autochthone mineralische Sedimentanteile}

\subsubsection{Diatomeen-Frusteln}

Mit dem Nachweis der Gattungen Melosira bzw. Aulacoseira in den Seesedimenten von Messel (GOTH 1990) und Eckfeld (WILDE et al. 1993, ScHILLER 1999, diese Arbeit) läßt sich die stratigraphische Reichweite lakustriner Diatomeen bis ins Mitteleozän hinein verfolgen. Neueste Erkenntnisse aus den nicht marinen Sedimenten der Tarahumara Formation (Mexiko) machen jedoch wahrscheinlich, daß einzelne Diatomeen-Gattungen bereits in der Oberkreide limnische Habitate besiedelten (CHACÓN-BACA et al. 2002).

Im Vergleich zu anderen lakustrinen Tertiärvorkommen Mitteleuropas, wie beispielsweise Sieblos/Rhön (Unteroligozän; MARTINI \& SCHRADER 1988, SCHILLER \& LANGE-BERTALOT 1997, SCHILLER 1998), Enspel/Westerwald (Oberoligozän; SCHILLER 2000) und Rott/Siebengebirge (Oberoligozän; MöRS 1995), zeichnet sich die Diatomeen-Flora von Eckfeld durch eine ungewöhnlich geringe Diversität aus. Neben einzelnen Individuen der pennaten Gattungen Eunotia und Pinnularia ist eine noch nicht bestimmte Form der Gattung Aulacoseira Teil des Artenspektrums (SCHILLER 1999). In den diatomeenreichen Lagen der Mikrofazieszonen 3b und 3d dominieren jedoch bei weitem die zentrischen Frusteln von Aulacoseira cf. granulata (Anhang 2-11, Fig. 03).

Tab. 21: Taxonomische Stellung von Aulacoseira granulata

Aulacoseira granulata (EHRENBERG) SIMONSEN 1979

Ordnung:

Familie:

Gattung:
Aulacoseirales CRAWFORD 1990

Aulacoseiraceae CRAWFORD 1990

Aulacoseira THWAITES 1848 
Die Art granulata wurde früher unter den Gattungsnamen Melosira (sensu lato) gestellt, der nach CRAWFORD (1975) vier unterschiedliche morphologische Gruppen zusammenfaßte. Nach einer umfangreichen Neukombination von SIMONSEN (1979) ist die frühere Gruppe um $M$. granulata nunmehr der Gattung Aulacoseira zugehörig (siehe auch HAWORTH 1988, ROUND et al. 1990, KRAMMER \& LANGE-BERTALOT 1991). Der Artname Melosira granulata wird allerdings von vielen Autoren auch weiterhin als Synonym verwendet.

Rezent-Formen von Aulacoseira granulata (Melosira granulata) leben kosmopolitisch als Plankter in Flüssen, Teichen und Seen (CHOLNOKY 1968, KRAMMER \& LANGE-BERTALOT 1991). Sie zeigen die in Tab. 22 zusammengefaßten, ökologische Toleranzen.

Tab. 22: Ökologische Anforderungen von Aulacoseira granulata

\begin{tabular}{|c|c|c|}
\hline Salinität & $\begin{array}{l}\text { - oligohalob, meioeuryhalin } \\
\text { - } 0 \% 0-5 \% \text { o }\end{array}$ & $\begin{array}{ll}\text { - } & \text { PANKOW (1976) } \\
\text { - } & \text { MÜLLER-WILMES (1989) } \\
\end{array}$ \\
\hline Temperatur & - optimale Entwicklung bei etwa $19^{\circ} \mathrm{C}$ & - STOERMER \& LADEWSKI (1976) \\
\hline Trophie & - eutroph & $\begin{array}{l}\text { - HUSTEDT }(1945,1957), \text { NYGAARD } \\
\text { (1956), ROUND (1981), KRAMMER \& } \\
\text { LANGE-BERTALOT (1991) }\end{array}$ \\
\hline & - mit Einschränkung eutroph & - CHOLNOKY (1968) \\
\hline & - $\quad$ alkaliphil & - $\quad$ NYGAARD (1956), FogED (1980) \\
\hline $\mathrm{pH}$-Wert & $\begin{array}{l}\text { - } \mathrm{pH} 6,3-\mathrm{pH} 9,0 \text {; optimale Entwicklung } \\
\text { bei } \mathrm{pH} 7,9-\mathrm{pH} 8,2\end{array}$ & $\begin{array}{l}\text { - V. D. WERFF \& HULS (1957-1974), } \\
\text { CHOLNOKY (1968) }\end{array}$ \\
\hline Si-Gehalt & - $\operatorname{hoch}(>7 \mathrm{mg} / \mathrm{l})$ & - $\quad$ MÜLLER-WILMES (1989) \\
\hline
\end{tabular}

\subsubsection{Chrysophyceen-Zysten}

Chrysophyceen sind eine diverse Gruppe von vorwiegend planktonisch lebenden Süßwasser-Algen, die angesichts ihres hohen Gehaltes an Karotinoiden auch als Goldalgen bezeichnet werden (STARMACH 1985, WILDE et al. 1993). Die verkieselten Zysten von Chrysophyceen sind endoplasmatisch gebildete Ruhestadien (Stomatozysten) und in Form, Größe und Skulptur vermutlich artspezifisch (SANDGREN 1991, DUFF et al. 1995). Als alternativer Morphotyp sichern sie das ganzjährige Fortbestehen der Algen, wenn der vegetative Wachstumsprozeß über einen Teil des Jahres hinweg eingeschränkt ist (SANDGREN 1988). In anoxischen Sedimenten scheinen die Ruhestadien allerdings auch noch nach mehreren Jahrzehnten lebensfähig zu sein (SANDGREN 1991). Allerdings konnte bislang nur ein sehr geringer Teil der fossilen ZystenMorphotypen rezenten Chrysophyceen-Gattungen zugeordnet werden (SMOL 1988, SCHILLER 2000).

WUTTKE \& RADTKE (1993) beschreiben kugelige Hohlräume mit einem Durchmesser zwischen $5 \mu \mathrm{m}$ bis $10 \mu \mathrm{m}$ aus stratigraphisch nicht lokalisiertem Probenmaterial des Eckfelder Grabungsprofils. Sie werden von den Autoren als Abdrücke von Chrysophyceen-Zysten gedeutet. Innerhalb des Bohrprofils von 1980 belegt ZOLITSCHKA (1993) das spärliche bis regelmäßige Vorkommen unbekannter Zysten zwischen Bohrmeter 43,7 und 33,6. Im Rahmen dieser Arbeit konnten die Eckfelder Stomatozysten erstmalig elektronenmikroskopisch nachgewiesen und der "Stomatocyst 133" (sensu DuFF et al. 1995) zugeordnet werden (Anhang 211, Fig. 05). 
Tab. 23: Taxonomische Stellung von "Stomatocyst 133"

\begin{tabular}{ll}
\hline "Stomatocyst 133" & \\
\hline Stamm: & Heterokontophyta CAVALIER-SMITH 1981 \\
Klasse: & Chrysophyceae PASCHER 1931 \\
Hilfsfamilie: & Chrysostomataceae CHODAT 1922 \\
\hline Beschreibung in: & DUFF et al. (1995, S. 111, Fig. 107) \\
\hline
\end{tabular}

Beschreibung: Kugelige bis leicht ovale Form mit einer Breite zwischen $12 \mu \mathrm{m}$ und $13 \mu \mathrm{m}$ und einer Länge zwischen $13,5 \mu \mathrm{m}$ und $14,5 \mu \mathrm{m}$ (Länge:Breite $=1,0$ bis 1,2 ). Die $1 \mu \mathrm{m}$ bis $2 \mu \mathrm{m}$ große Pore ist von einem deutlich abgegrenzten Hals mit einem Apikaldurchmesser von rund $6 \mu \mathrm{m}$ umgeben. Der komplex aufgebaute Hals besteht aus einem kegelförmigen Primärkragen und einem trichterförmigem Sekundärkragen, auf dessen Innenseite zusätzlich spiralartige, unterbrochene Kämme ("spiralling ridges", sensu DuFf et al. 1995) auftreten. Die Zyste entspricht der von DUFF \& SMOL (1994) und DUFF et al. (1995) beschriebenen "Forma C" von "Stomatocyst 133". Gelegentlich sind parallele Längsstreben ("collar struts", sensu DuFf et al. 1995) im Übergangsbereich von Zystenhals zu Zystenkörper zu erkennen. Der Körper ist mit echinaten, $0,5 \mu \mathrm{m}$ bis $1,5 \mu \mathrm{m}$ hohen Dornen und wahllos orientierten, teils gebogenen Kämmen (Länge: 1,0 bis 3,0 $\mu \mathrm{m}$; Höhe: $0,5 \mu \mathrm{m}$ bis $1,0 \mu \mathrm{m}$ ) besetzt. In einigen Fällen ist die Skulptierung nur schwach ausgeprägt und vorwiegend auf die posteriore Hemisphäre beschränkt. Diese Zysten-Form scheint morphologisch zu der weitgehend unornamentierten "Forma A" von DuFf et al. (1995) überzuleiten.

Abgrenzung: RYBAK et al. (1991) beschreiben einen ähnlichen Morphotyp (Cyst \#39), der mit einem Durchmesser von maximal $8,4 \mu \mathrm{m}$ jedoch insgesamt etwas kleiner ist, als die in Eckfeld gefundene Form.

Biologische Affinität: Die rezente "Stomatocyst 133" wird vermutlich von einer OchromonasArt gebildet (DuFf et al. 1995). Ochromonas ist eine gut bekannte Chrysophyceen-Gattung mit etwa 70 rezenten Arten, die kosmopolitisch in Teichen und Tümpel, aber auch in leicht salzigen Gewässern auftreten. Die Algen leben freischwimmend und bilden nur gelegentlich und vorübergehend kleine, lockere Kolonien (STARMACH 1985).

In Eckfeld treten Chrysophyceen-Zysten vereinzelt in den Diatomeenlagen der MFZ 3b: Aulacoseira-Fazies auf. Innerhalb geringmächtiger $(<40 \mu \mathrm{m})$ Tonzwischenschichten kommen aber auch Massenansammlungen in Form von $<1 \mathrm{~mm}$ langen, ein bis fünf Zysten-Durchmesser mächtigen Linsen vor (Anhang 2-2, Fig. 08 bzw. Anhang 2-3, Fig. 01). Die Zystenkonzentrationen wurden allerdings nur selten und lediglich in den Proben BL/A-F1.2 und BL/A-F1.8 nachgewiesen. Sie sind keinesfalls eine regelmäßige Komponente der rhythmischen Sedimentation aus Diatomeen- (LT 3b-1) und organisch-reichen Tonlagen (LT 3b-2).

Bei einem korrodierten Einzelexemplar aus der hangenden MFZ 3c: Huminit-Opal-Fazies handelt es sich nach der Form und Größe ebenfalls um "Stomatocyst 133" (Anhang 2-11, Fig. 06). 


\subsubsection{Spongilliden-Nadeln}

Fossile Süßwasserschwämme sind erst seit dem Jura (Portlandium) bekannt und werden nur vergleichsweise selten gefunden (GRUBER 1994). Allerdings ist ihr Vorkommen aus vielen Tertiärvorkommen Mitteleuropas vorwiegend durch Skleren belegt, wie beispielsweise aus Messel (Mitteleozän; HeIL 1964, MARTINI \& RIETSCHEL 1978, MülLER et al. 1982, WUTTKE 1988, GotH 1990, RICHTER \& WUTTKE 1995, LIEBIG 1998, RICHTER \& WUTTKE 1999), aus Sieblos/Rhön (Unteroligozän; MARTINI \& SCHRAdER 1988, MARTINI \& ROTHE 1993, MARTINI \& SCHILLER 1995, SCHILLER 1998), aus Enspel/Westerwald (Oberoligozän; SCHILLER 2000) und aus Rott/Siebengebirge (Oberoligozän; MÖRS 1995, BRAUN 2001).

In Eckfeld wurden gerade oder leicht gebogene Monaxone innerhalb der Mikrofazieszonen 3b: Aulacoseira-Fazies und 3d: Aulacoseira-Opal(CT)-Fazies nachgewiesen. Die 200um bis $350 \mu \mathrm{m}$ langen und rund $10 \mu \mathrm{m}$ dicken Amphioxe sind glatt bis lediglich schwach bedornt. Sie treten isoliert oder als Bündel von etwa zehn Nadeln sowohl in den diatomeenreichen Lagen (LT 3b-1 bzw. LT 3d-1) als auch in den tonigen Zwischenschichten (LT 3b-2 bzw. LT 3d-2) auf (Anhang 2-11, Fig. 07). Der Zentralkanal angeschnittener Skleren ist im BSE-Bild deutlich sichtbar (Anhang 2-5, Fig. 05). Innerhalb der Mikrofazieszone 3e: Tetraedron-Opal(CT)-Fazies wurden die kieseligen Spongien-Nadeln vollständig aufgelöst und sind nur noch als Negative im opalreichen Sediment zu erkennen. Ebenso wie die Abdrücke von Diatomeen-Schalen beinhalten auch die Hohlräume gelöster Spiculae häufig Opal(CT)-Lepisphären mit einem Durchmesser von maximal $3 \mu \mathrm{m}$.

Anhand isolierter Monaxone lassen sich Süßwasserspongien artlich nicht bestimmen (SCHILlER 2000, ReITNER, frndl. mündl. Mitt. 2002). Da in Eckfeld bisher weder taxonomisch relevante Gemmulae bzw. Gemmulae-Belagsskleren als auch Mikroskleren gefunden wurden, können die Skelettnadeln nach derzeitigem Kenntnisstand lediglich den Spongillidae GRAY 1867 zugeordnet werden (GRUBER 1994). Bei der Familie der Spongilliden handelt es sich jedoch sicherlich um eine künstliche Gruppierung phylogenetisch unterschiedlicher Taxa (VOLKMER-RIBEIRO 1990, VOLKMER-RIBEIRO \& REITNER 1991).

Tab. 24: Taxonomische Stellung der Spongilliden

\begin{tabular}{ll} 
Spongillidae GRAY 1867 & \\
\hline Stamm: & Porifera GRANT 1872 \\
Klasse: & Demospongiae SoLLAS 1875 \\
Unterklasse: & Ceractinomorpha (Monaxonida) LÉVI 1973 \\
Ordnung: & Haplosclerida TOPSENT 1898
\end{tabular}

Rezent sind Süßwasserschwämme mit etwa 200 Arten vertreten. Sie leben sessil als Filtrierer und bevorzugen gut durchlüftete, meist halbschattige Habitate. Das Substrat, in der Regel unter Wasser befindliche Steine und Wurzeln, aber auch auf dem Wasser schwimmende Äste, bestimmt die Spongilliden-Gestalt. Meist ist der Schwammkörper fladen- bis krustenförmig und nur wenige Millimeter hoch (WEISSENFELS 1989, GRUBER 1994). Süßwasserschwämme 
tolerieren rezent eutrophe bis fast klare Gewässer und sind somit als Paläotrophieanzeiger weitgehend ungeeignet (WEISSENFELS 1989, ZOLITSCHKA 1993).

Bemerkenswert ist der relative Reichtum an Spongien-Nadeln in den uferfernen Seeablagerungen von Eckfeld. Da insbesondere die sklerenführenden Diatomeenlagen der Mikrofazieszonen $3 b$ und $3 d$ keinerlei Hinweise auf eine Sedimentverlagerung von den Randbereichen des Sees in Richtung Beckenmitte erkennen lassen, ist davon auszugehen, daß die Schwammkörper oftmals treibenden Pflanzenteilen ansaßen und mit innen über den See drifteten. Angesichts der steilen, durch zahlreiche Uferabbrüche gekennzeichneten Hänge (vgl. Kap. 7.1) bot der sicherlich schmale Litoralgürtel nur wenige Standorte für Spongilliden. Das Ansitzen an schwimmenden pflanzlichen Substraten kann möglichenfalls als eine spezielle "Überlebensstrategie" von Schwämmen im Eckfeld-See gewertet werden.

\subsection{Autochthone organische Sedimentanteile}

\subsubsection{Alginit}

Tab. 25: Position von Alginit innerhalb der Mazeralklassifikation

\begin{tabular}{|c|c|c|c|}
\hline Mazeral-Gruppe & Mazeral-Subgruppe & Mazeral & Mazeral-Typ \\
\hline Liptinit & & Alginit & $\begin{array}{c}\text { Telalginit } \\
\text { (Botryococcus) } \\
\text { Lamalginit } \\
\text { (Tetraedron, Lamalginit unstrukturiert) }\end{array}$ \\
\hline
\end{tabular}

\section{Telalginit}

Der Begriff Telalginit wurde von HUTTON (1987) eingeführt und als Synonym für Alginit A (HUTTON et al. 1980) verwendet. Telalginit (bzw. Alginit A) ist definiert als: "... alginite, derived from large colonial or thick-walled unicellular algae [...], with strong fluorescence at low rank and with distinctive external structure, and in many cases internal botanical structures when viewed in sections perpendicular to bedding" (HUTTON 1987, S. 208). Gemäß dieser Definition ist Telalginit mit der Chlorophyceen-Gattung Botryococcus in den zentralen Seesedimenten von Eckfeld vertreten.

Botryococcus. Die meisten fossil auftretenden Überreste der koloniebildenden Grünalge Botryococcus werden heute mit der rezenten Art Botryococcus braunii KüTZING 1849 verglichen (KOMÁREK \& MARVAN 1992, WILDE et al. 1993). KüTZING's kurze Diagnose liefert nach KOMÁREK \& MARVAN (1992) jedoch nur unzureichende Angaben über wichtige Merkmale zur Differenzierung einzelner Morphotypen. Heutzutage ist es schwierig, den Typus zu rekonstruieren, auf den sich KÜTZING im Original bezieht. Dieses mag die Ursache dafür sein, daß spätere Autoren den Artnamen braunii heranzogen, um sehr unterschiedliche Botryococcus-Formen zu benennen. Beschreibungen rezenter Botryococcus-Populationen beruhen unter anderem auf feinmorphologischen Merkmalen (KOMÁREK \& MARVAN 1992, BATTEN \& GRENFELL 1996), die fossil kaum erhaltungsfähig sind (siehe auch COOK \& KANTSLER 1982).

Aus den obengenannten Gründen wird die in Eckfeld auftretende Art an dieser Stelle als Botryococcus cf. braunii beschrieben. 
Ordnung:

Familie:

Gattung:
Chlorococcales MARCHAND 1895

Botryococcaceae WILLE 1909

Botryococcus KÜTZING 1849

Beschreibung in:

KOMÁREK \& FOTT $(1983$, S. 378)

Botryococcus ist rezent nahezu kosmopolitisch zwischen den Tropen und den subarktischen Klimazonen verbreitet. Die Alge kommt vorwiegend in langsam fließenden Süßgewässern und lakustrinen Milieus vor (GUY-OHLSON 1992, TRAVERSE 1992, TAYLOR et al. 1998), toleriert aber auch schwach saline Bedingungen (DE DECKKER 1988, BATTEN 1996a). Nach WETZEL (1983) ist Botryococcus häufig in pH-neutralen bis schwach alkalischen Seen zu finden, kann aber grundsätzlich über einen weiten pH-Bereich zwischen 4 und über 9 auftreten (WAKE \& HILLEN 1980). Botryococcus scheint ein typischer Phytoplanktonvertreter in oligotrophen bis mesotrophen Wasserkörpern zu sein (ROUND 1981, KOMÁREK \& MARVAN 1992, TYSON 1995, BATTEN 1996a). Andererseits wiesen RUTTNER (1952) bzW. ROUND \& BROOK (1959) die Alge mit unterschiedlicher Häufigkeit in eindeutig eutrophen Seen (Telaga Pasir und Tjigombong-Stausee, Java bzw. Lake Cullin, Irland) nach. Vor diesem Hintergrund erscheint Botryococcus als Trophieanzeiger ungeeignet (siehe auch AARONSON et al. 1983, WILDE et al. 1993).

Der Lebenszyklus rezenter Botryococcus-Arten beginnt mit der longitudinalen Teilung der birnenförmigen Autospore in zwei Tochterzellen, gefolgt von einer zweiten Teilung im rechten Winkel dazu. Die Zellen sitzen in becherförmigen Kammern ("cups"), die bei jeder Zellteilung neu gebildet werden und so ein im Querschnitt lagig aufgebautes Kammer-Gerüst erzeugen. Eine fortgesetzte Wiederholung des Teilungsprozesses führt zu einer unregelmäßigen Anordnung kleinerer Zellgruppen an der Oberfläche der Kolonie ("compound colony") und zu dem charakteristischen botryoidalen Erscheinungsbild (Anhang 2-12, Fig. 01). Erreicht die Kolonie eine Größe von 40 bis 60 Zellen teilt sie sich für gewöhnlich und bildet entweder isolierte Tochter-Kolonien ("unbranched compound colonies") oder ein über Gallert-Stränge verzweigtes Kolonie-Aggregat ("branched compound colony") (AARONSON et al. 1983, KOMÁREK \& FOTT 1983, GUY-OHLSON 1992, BATTEN \& GRENFELL 1996).

Während der Frühdiagenese werden der Zellinhalt und die zellulosereiche Zellwand zerstört (BATTEN \& GRENFELL 1996). Neuere Untersuchungen haben jedoch gezeigt, daß Teile der Zellkammern durch bedeutende Gehalte eines hoch aliphatischen Biomakromoleküls (Algaenan) charakterisiert sind, das aufgrund seiner Resistenz gegenüber chemischer Degradation (BERKALOFF et al. 1983) und seiner selektiven Erhaltung während des Fossilisierungsprozesses auch als PRB (Polymère Resistant de Botryococcus) bezeichnet wird (LARGEAU et al. 1984, LARGEAU et al. 1986, KADOURI et al. 1988, GELIN et al. 1994, BATTEN \& GRENFELL 1996).

Der Fossilbericht von Botryococcus reicht nach TAPPAN (1980) und GUY-OHLSON (1998) möglicherweise bis ins Präkambrium zurück. Während die in den permischen "boghaed" Kohlen Frankreichs, Australiens und Südamerikas häufig auftretenden Algen-Gattungen Pila und Reinschia sowie die mesozoische Epipolaia corymbus gemeinhin als Fossilsynonyme für 
Botryococcus anerkannt sind (TemPERLEY 1936, TRAVERSE 1955, AlPERN 1981, TeichmüLleR 1989, BATTEN \& GRENFELL 1996), ist die botanische Affinität zu Gloeocapsomorpha beispielsweise aus den ordovizischen Kuckersiten Estlands umstritten (DERENNE et al. 1991, TYSON 1995, BATTEN \& GRENFELL 1996). Die stratigraphische Reichweite von Botryococcus ist daher vermutlich geringer als von TAPPAN (1980; Präkambrium-rezent) und TRAVERSE (1955; Ordovizium-rezent) angenommen (ZIPPI 1998).

Abgesehen von den mitteleozänen Maarsedimenten von Eckfeld (LUTZ 1993d, WILDE et al. 1993, NICKEL 1994, 1996) ist Botryococcus aus einer ganzen Reihe weiterer isolierter Tertiärvorkommen Deutschlands bekannt, beispielsweise aus den unteroligozänen SieblosSchichten/Rhön (MARTINI \& ROTHE 1988, 1993, HOTTENROTT 1998), den oberoligozänen Seeablagerungen von Enspel/Westerwald (CLAUSING 1998, MüNTER 1999, SCHILLER 2000) und den obermiozänen Sedimenten des Ries-Sees (DEHM et al. 1977, V. D. BRELIE 1977, HOLLERBACH et al. 1977). In den mitteleozänen Ölschiefern von Messel treten einzelne Botryococcus-Kolonien fast überall im Sediment auf und bilden teilweise auch lagige oder linsige Anreicherungen (GoTH 1990). Trotz des gehäuften Vorkommens in einzelnen Profilabschnitten spielt die Grünalge jedoch in der Gesamtbilanz der Primärproduktion nur eine untergeordnete Rolle (JANKOWSKI \& LITTKE 1986, GOTH 1990). Im Gegensatz dazu ist Botryococcus in den oberoligozänen Seesedimenten von Rott/Siebengebirge deutlich häufiger vertreten (MÖRS 1995).

In den beckenzentralen Eckfelder Schwarzpeliten sind Botryococcus-Kolonien aufgrund ihrer leuchtend gelben Fluoreszenzfarbe und ihrem charakteristischen botryoidalen Habitus leicht nachzuweisen. Insbesondere in Sedimentabschnitten, die reich sind an frühdiagenetisch gebildetem Opal ist die Internstruktur einzelner Kolonien weitgehend erhalten geblieben (Anhang 211, Fig. 08). Es lassen sich trichterförmige Zellbecher mit einem Durchmesser zwischen $6 \mu m$ und $9 \mu \mathrm{m}$ erkennen, die unregelmäßig über die gesamte Kolonie verteilt sind. Größere Kolonien beinhalten rund 50 bis 100 einzelne Zellkammern. Es kommen aber auch sehr kleine Kolonien mit < 10 sichtbaren "cups" vor, die offensichtlich nur wenige Zellteilungsphasen durchlaufen haben (Anhang 2-11, Fig. 08 bzw. Anhang 2-12, Fig. 02). Die Alge ist nahezu über das gesamte Profil hinweg vertreten und zeigt mehrere Häufigkeitsmaxima zwischen rund 4 Vol.\% und 7 Vol.\% Lediglich in den rein mineralischen Laminiten der LFZ 1 und in zwei weiteren Proben der hangenden organisch-reichen Sedimente (KS5 und KS17) wurde sie nicht registriert. Botryococcus-Kolonien sind typischerweise dispers und regellos im Sediment verteilt. In Profilabschnitten, die sich durch einen generellen Reichtum an Botryococcus auszeichnen, tritt die Alge darüber hinaus häufig in $50 \mu \mathrm{m}$ bis $500 \mu \mathrm{m}$ mächtigen Bereichen konzentriert auf. Reine Botryococcus-Lagen sind dagegen vergleichsweise selten, meist nicht über die ganze Schliffbreite aushaltend und nur wenige Koloniedurchmesser mächtig.

NiCKEL $(1994,1996)$ wies auf das Vorkommen von mindestens zwei Kolonie-Varianten in den Eckfelder Maarsedimenten hin, die sich vorwiegend durch ihre Größe unterscheiden. Die als "kleine Botryococcus-Kolonien" bezeichneten Formen zeigen undeutlich ausgebildete becherartige Strukturen, in denen die Einzelzellen der Kolonie saßen. Im Gegensatz dazu weisen die sogenannten "großen Botryococcus-Kolonien" einen klaren, blumenkohlartigen Habitus mit deutlich größeren Zellbechern auf. Um diesen Befund mit numerischen Verfahren (Cluster-Analyse) zu verifizieren, wurden die Größen von insgesamt 3280 BotryococcusKolonien aus 17 ausgewählten Kornpräparaten der Bohrungen E1/96 und E2/96 unter dem 
Fluoreszenzmikroskop vermessen und jeweils $10 \mu \mathrm{m}$ umfassenden Größenklassen zugeordnet. Es sollte der Frage nachgegangen werden, ob sich in den untersuchten Proben eine statistische Bevorzugung bestimmter Koloniedurchmesser abzeichnet bzw. ob möglichenfalls privilegierte Größen zusammen in einem Sedimentabschnitt auftreten oder sich gegenseitig ausschließen. Bei der Größenverteilung ist sicherlich ein gewisser Streuungseffekt zu berücksichtigen, der durch die Kompaktion einzelner Botryococcus-Kolonien bedingt ist, aber auch durch die Erfassung unterschiedlicher Anschnittsebenen im Sediment. Die Ergebnisse geben also lediglich Tendenzen wieder, die für sich genommen jedoch auffallend eindeutig sind (Abb $20 \mathrm{~S}$ ). Bei der Vermessung wurden dispers im Sediment verteilte Botryococcus-Kolonien getrennt von lagenweise angereicherten Kolonien erfaßt. Aus den Untersuchungen ergeben sich folgende Aussagen:

- Die Cluster-Analyse (Abb. $20 \mathrm{~T}$ ) zeigt zwei eindeutig voneinander getrennte ProbenCluster. Cluster I beinhaltet Proben, die durch das Auftreten sehr kleiner BotryococcusKolonien mit wenigen (meist unter zehn) und im Fluoreszenz-Bild oft nur undeutlich erkennbaren Zellkammern gekennzeichnet sind. Dieser Kolonie-Typ entspricht allem Anschein nach den von NiCKEL $(1994,1996)$ beschriebenen "kleinen Botryococcus-Kolonien". Die weitaus

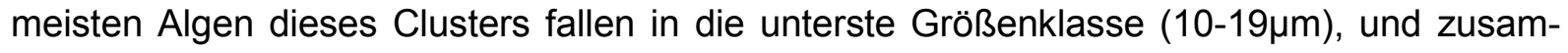
men mit der darauffolgenden Klasse $(20-29 \mu \mathrm{m})$ werden zwischen $84 \%$ und $99 \%$ der registrierten Kolonien erfaßt. Diese geringe Größenvarianz gilt insbesondere für BotryococcusKolonien, die in Lagen konzentriert auftreten (Abb. 21). Hier fallen zwischen 96\% und 100\% der Algen in die ersten beiden Größenklassen $(10 \mu \mathrm{m}-29 \mu \mathrm{m})$.

Der ausnahmslos geringe Durchmesser der Kolonien deutet auf äusserst kurze Wachstumsphasen der Alge hin (siehe auch GUY-OHLSON 1998), in denen sich offensichtlich nur eine Botryococcus-Generation entwickeln konnte. Dieses war insbesondere während Algenblüten der Fall. Dispers im Sediment verteilte Botryococcus-Kolonien können dagegen auch Ruhestadien darstellen, in denen die Wachstumsgeschwindigkeit der Alge reduziert war (vgl. TAPPAN 1980).

- Im zweiten Cluster werden Proben zusammengefaßt, die Botryococcus-Kolonien von variabler Größe führen. Die in den Histogrammen abgebildeten Größenvariationen (Abb. 20 $C, E, H, L$, und $Q$ ) zeigen annähernd das Bild einer Normalverteilung, bei der das Maximum im Vergleich zum Cluster I deutlich in Richtung der größeren Koloniedurchmesser verscho-

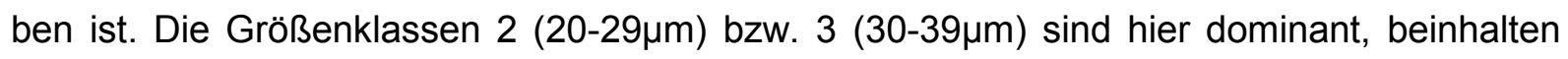
zusammen aber nur rund die Hälfte der ermittelten Gesamthäufigkeiten (Abb. $20 \mathrm{~S}$ ). Es kommen sowohl kleinere Kolonien $(10-19 \mu \mathrm{m})$ in nennenswerter Häufigkeit vor, als auch größere "unbranched compound colonies" mit einem Durchmesser von > 100 $\mu \mathrm{m}$.

Im fluoreszenzmikroskopischen Bild vermitteln Botryococcus-reiche Lagen vielfach bereits subjektiv den Eindruck von zwei bevorzugten Koloniedurchmessern. Tatsächlich konnte eine tendenziell bimodale Verteilung von Koloniegrößen nachgewiesen werden. Innerhalb der Botryococcus-Lagen dieses Probenclusters schließt sich einem Größenmaximum zwischen

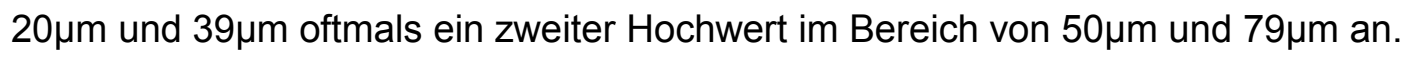


6 Bestandteile der zentralen Seesedimente
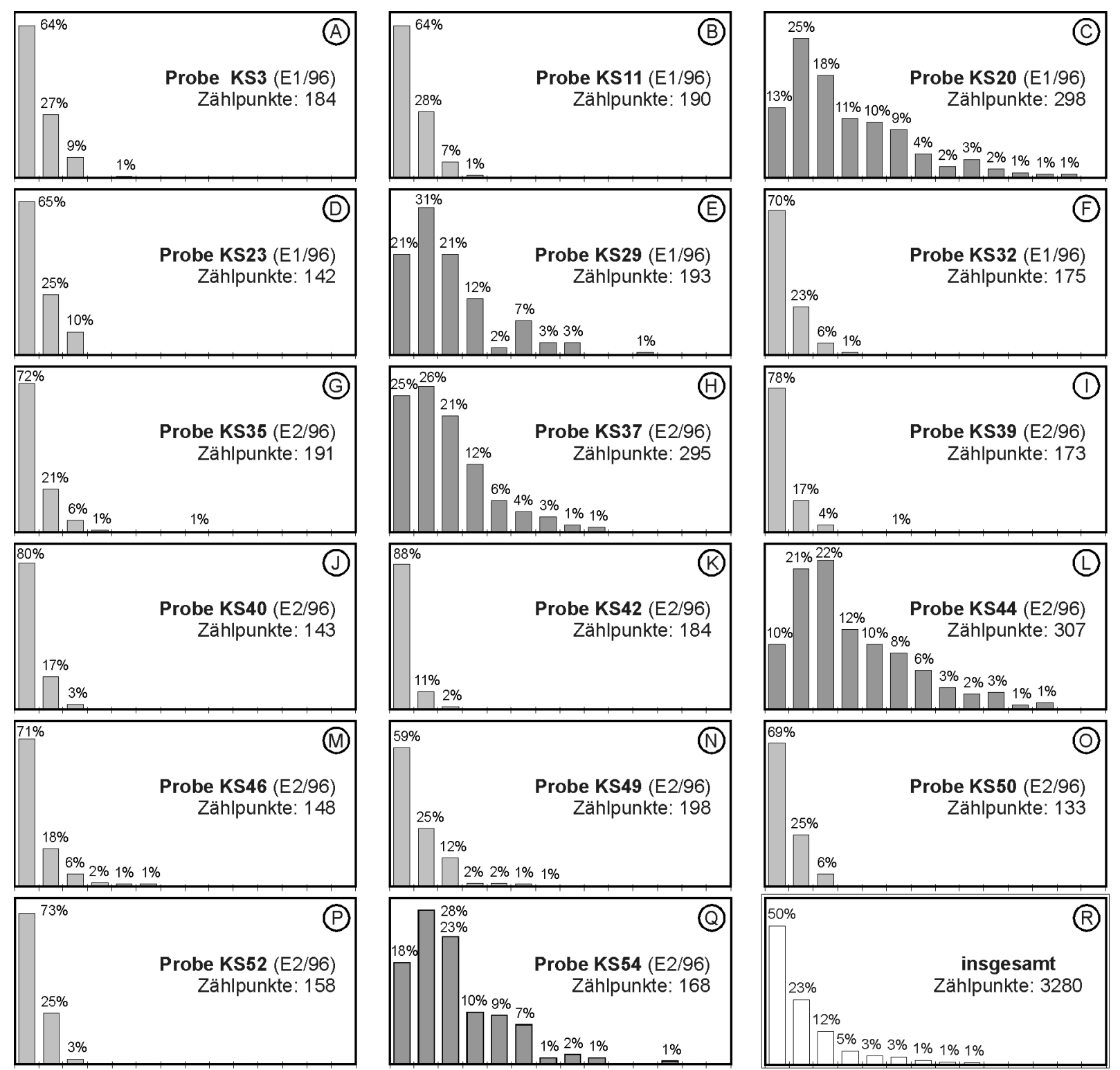

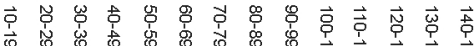

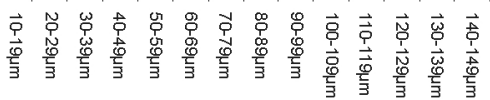

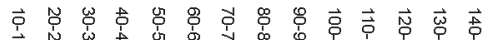

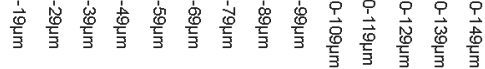

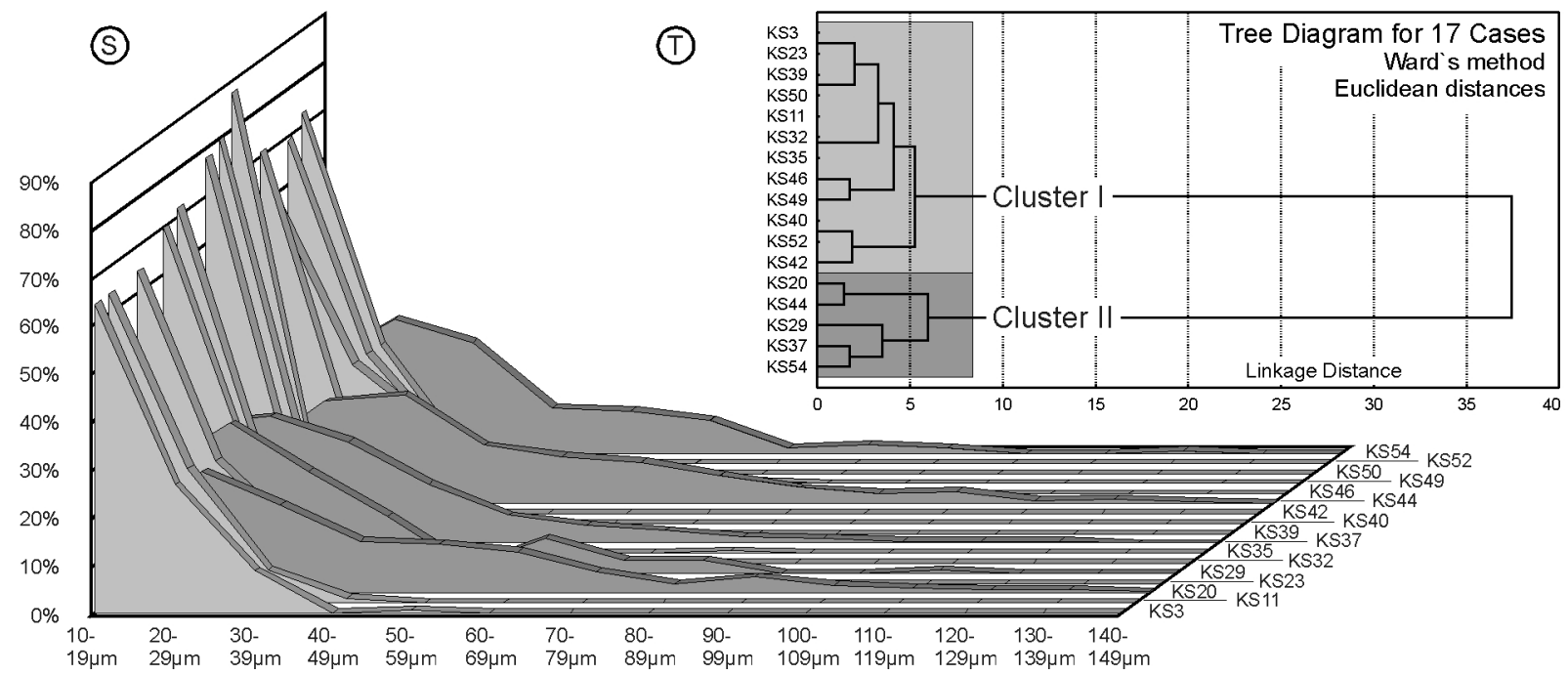

Abb. 20: Koloniegrößen von Botryococcus in ausgewählten Proben der LFZ 3: Biogene Laminite. 

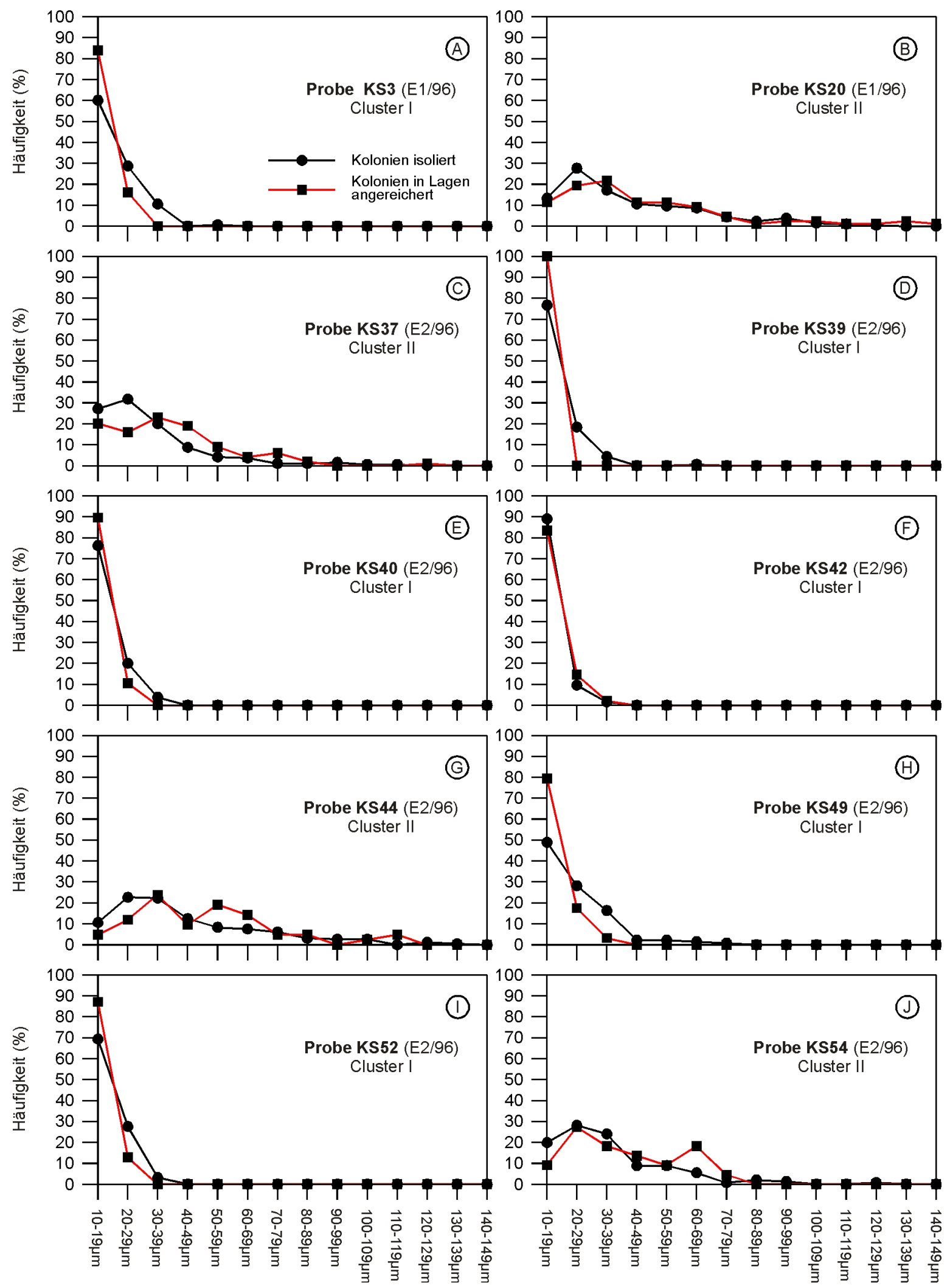

Abb. 21: Koloniegrößen von Botryococcus (vgl. Abb. 20) getrennt nach isolierten und lagenweise angereicherten Vorkommen.

Verglichen mit dem Cluster I können hier entsprechend längere Wachstumsperioden angenommen werden (siehe auch GUY-OHLSON 1998). Unter natürlichen Bedingungen verdoppelt sich die Biomasse rezenter Botryococcus-Arten während Phasen exponentiellen Wachstums in einem Zeitraum von 7 bis 10 Tagen (TAPPAN 1980, GUY-OHLSON 1998). Die 


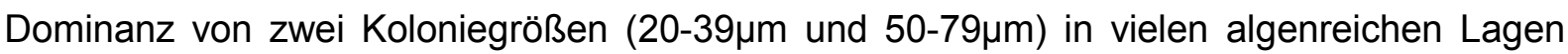
läßt auf Algenblüten schließen, die lange genug andauerten um mindestens zwei Lebenszyklen von Botryococcus zu entwickeln. Während längerer Wachstumsphasen könnte es aber auch zu einer vermehrten Abspaltung kleinerer Tochterkolonien gekommen sein, als die Mutterkolonien kritische Größen erreicht hatten (s.o.).

- Innerhalb der untersuchten Proben stehen die Koloniedurchmesser in keiner signifikanten Relation zu der jeweiligen Gesamthäufigkeit von Botryococcus im Sediment. Beispielsweise sind die "kleinen Kolonien" des Clusters I sowohl in verhältnismäßig Botryococcusreichen, als auch in auffallend -armen Profilabschnitten vertreten (Abb. 20 und Anlage 2). Ähnliches gilt auch für die Proben des zweiten Clusters. Allerdings liegen hier drei der fünf Proben (KS20, KS37 und KS44) im stratigraphischen Niveau ausgesprochener Botryococcus-Maxima.

- Bei einer angenommenen Sedimentationsrate von 0,4 - 0,5mm/a (NEGENDANK et al. 1982, MINGRAM 1998) und unter der Voraussetzung, daß die Größenvariationen von Botryococcus-Kolonien annähernd mit der Länge ihrer Wachstumsperioden korreliert sind (siehe oben), zeigen zumindest die $1 \mathrm{~cm}$ bis $2 \mathrm{~cm}$ Sedimentsäule umfassenden Kornschliffproben des Clusters I keinerlei Hinweise auf saisonal gesteuerte Schwankungen in der Botryococcus-Ontogenese. Der ausnahmslos kleine Durchmesser sowohl von dispers im Sediment verteilten als auch von lateral angereicherten Kolonien deutet vielmehr auf Umweltbedingungen hin, die bezogen auf die Produktivität der Alge über mehrere Jahre bis Jahrzehnte weitgehend stabil blieben. Zwar kam es zu sporadischen Massenentfaltungen von Botryococcus, eine Jahresrhythmik ist hierbei jedoch nicht nachzuweisen.

\section{Lamalginit}

Nach einer Neudefinition von HUTTON (1987) wurde der bis dahin gültige Begriff Alginit B (HUTTON et al. 1980) durch den Terminus Lamalginit ersetzt und als "... alginite, derived from small, unicellular or thin-walled, colonial planktonic or benthonic algae [...], with weak to moderate (rarely intense) fluorescence at low rank and a lamellar form with little recognizable structure in sections perpendicular to bedding" beschrieben (HUTTON 1987, S. 208). Nach dieser auf fluoreszenzmikroskopischen Untersuchungen basierenden Definition ist die coccale Grünalge Tetraedron mit Einschränkung dem Mazeral-Typ Lamalginit zuzuordnen. Zwar sind die dünnwandigen Einzelzellen der Alge in den untersuchten Proben oftmals stark kompaktiert, doch lassen sich häufig auch dreidimensional erhaltene Exemplare belegen, die keine lamellare Form aufweisen (siehe unten).

Neben Tetraedron wurde "unstrukturierter Lamalginit" als Mazeral-Typ klassifiziert. Die allgemeine Merkmalsarmut von "unstrukturiertem Lamalginit" bietet keine direkten Hinweise auf seine Vorläuferorganismen.

Tetraedron. Der Individuenreichtum der coccalen Grünalge Tetraedron ist bestimmend für die Abgrenzung der Mikrofazieszone 3e: Tetraedron-Opal(CT)-Fazies in den zentrumsnahen Bohrprofilen. Ab Bohrmeter 19,36 (Probe KS33) blieben die dünnwandigen, $8 \mu \mathrm{m}$ bis $11 \mu \mathrm{m}$ großen Einzelzellen häufig unkompaktiert innerhalb stark verkieselter Profilabschnitte erhalten. Für die botanische Zuordnung der Alge erwies sich die Auflicht-Fluoreszenzmikroskopie gegen- 
über der REM als die geeignetere Methode. An unverdrückten, gelbgrün bis rötlichgelb fluoreszierenden Exemplaren konnte der für Tetraedron minimum charakteristische, kissenförmige Habitus mit den gerundeten Zellwandpapillen in Form von "schnäuzchenartig hervorstehenden Ecken" (Gотн 1990, S.15) eindeutig belegt werden (Anhang 2-12, Fig. 03). Der Nachweis einer scrobiculaten bzw. retikulaten Oberflächenskulptur mittels Rasterelektronenmikroskopie gelang trotz intensiver Bemühungen nicht. Angesichts der frühdiagenetisch stark beeinflußten Sedimente von MFZ 3e (vgl. Kap. 4.3.5) ist dieser Umstand allerdings nicht überraschend. Zwar wurden innerhalb von Opallagen (LT 3e-1) häufig Partikel beobachtet, die nach Form und Größe durchaus Tetraedron zugewiesen werden können, doch verhinderte deren vollständige Umkrustung mit blättrigem Opal eine detaillierte feinmorphologische Analyse. Aufgrund der deutlichen fluoreszenmikroskopischen Befunde und unter Berücksichtigung der Ergebnisse anderer Bearbeiter (WILDE et al. 1993, ZOLITSCHKA 1993) kann der Nachweis von Tetraedron cf. minimum als häufiger Phytoplanktonvertreter in den obersten rund $20 \mathrm{~m}$ der zentralen Beckenfazies jedoch als gesichert gelten.

Tab. 27: Taxonomische Stellung von Tetraedron minimum

Tetraedron minimum (BRAUN) HANSGIRG 1888

\begin{tabular}{ll}
\hline Ordnung: & Chlorococcales MARCHAND 1895 \\
Familie: & Chlorellaceae BRUNNTHALER 1915 \\
Gattung: & Tetraedron KüTZING 1845 \\
\hline Beschreibung in: & Komárek \& Fott $(1983$, S. 697), Goth $(1990$, S. 17) \\
\hline
\end{tabular}

Tetraedron minimum vermehrt sich ausschließlich asexuell über Autosporen, die aus der zerrissenen Mutterzellwand in ein Gallertbläschen übertreten und erst später frei werden (KOMÁREK \& FOTT 1983, BATTEN 1996b). Die Zellwände bestehen aus einem resistenten hochaliphatischen Biopolymer (GoTH et al. 1988, GOTH 1990, BLOKKER et al. 1998). Ähnliche Biopolymere (Algaenan) wurden ebenfalls bei Botryococcus braunii gefunden (siehe oben).

Über die autökologischen Ansprüchen der rezenten Chlorophycee ist vergleichsweise wenig bekannt (WILDE et al. 1993, CLAUSING 2001). Tetraedron minimum kommt als Plankton und Periphyton in Teichen und Tümpeln vor (FOTT 1971, KOMÁREK \& FOTT 1983). Nach TYSON (1995) umfaßt die Gattung Tetraedron generell Taxa, die unter eutrophen Bedingungen leben (siehe auch ROUND 1981, BATTEN 1996b). JYOTHI et al. (1990) beschreiben eine Massenentfaltung von T. muticum im oligotrophen Wasser einer Zisterne in Hyderabad (Indien). Nach der taxonomischen Revision von KovÁčIK (1975) ist diese Algen-Form jedoch nicht mehr der Gattung Tetraedron zuzuordnen. In den vier Teilbecken des Toba-Sees (Sumatra) wurde Tetraedron minimum in Assoziation mit Botryococcus braunii und Melosira granulata (Aulacoseira granulata) nachgewiesen (RUTTNER 1952). T. minimum und M. granulata bilden überdies die Charakter-Formen dieser Lokalität. Im April 1929 lag der pH-Wert im Epilimnion des biochemisch geschichteten Sees zwischen 7,9 und 8,4 bei einer morgendlichen Wassertemperatur von rund $26^{\circ} \mathrm{C}$. 
Tetraedron minimum wurde fossil erstmalig in den mitteleozänen Seesedimenten von Messel nachgewiesen. Während in Messel (GOTH 1990) und teilweise auch im limnischen Oberoligozän von Enspel/Westerwald (SCHILLER 2000) nahezu reine Tetraedron-Lagen auftreten, ist die Alge in Rott/Siebengebirge (Oberoligozän) nur untergeordnet an der Gesamtbilanz der Primärproduktion beteiligt (MÖRS 1995). In bezug auf den Individuenreichtum von Tetraedron nehmen die Seeablagerungen von Eckfeld eine intermediäre Stellung zwischen Messel und Rott ein. Zwar treten keine monospezifischen Tetraedron-Lagen auf, doch sind in mehreren Proben (BL/TO(CT)-F1.1, BL/TO(CT)-F1.7, BL/TO(CT)-F2.3 bis BL/TO(CT)-F2.5) 20 $\mu \mathrm{m}$ bis $100 \mu \mathrm{m}$ mächtige Massenansammlungen nachzuweisen (Anhang 2-6, Fig. 08). Die Grünalge bildet in einigen Profilbereichen der MFZ 3e (Proben KS41, KS44, KS51 und KS54) ausgesprochene Häufigkeitsmaxima mit Werten von 6,4 Vol.\% bis 10,3 Vol.\%. In den Proben KS41, KS51 und KS54 ist sie sogar die dominante Chloropyceen-Form.

Der fluoreszenzmikroskopische Beleg von Tetraedron wird durch einen hohen Verkieselungsgrad der Sedimente begünstigt. Schwächer opalisierte Tonlagen sind dagegen durch das Auftreten von stark kompaktierten Alginiten mit einer Länge von rund 10 $\mu \mathrm{m}$ charakterisiert, die zwar nicht zweifelsfrei aber doch sehr wahrscheinlich ebenfalls von Tetraedron abzuleiten sind. Die vertikale Verteilung der nachgewiesenen Tetraedron-Zellen (Anlage 2) gibt also vermutlich nur einen sehr unvollständigen Eindruck von dem ursprünglichen Anteil der Alge an der Primärproduktion im Eckfeld-See.

Unstrukturierter Lamalginit. Im Gegensatz zu Tetraedron sind innerhalb von intern unstrukturierten Lamalginiten keine diskreten Algenzellen nachzuweisen. Es sind bis $500 \mu m$ lange und $2 \mu \mathrm{m}$ bis $10 \mu \mathrm{m}$ starke Lamellen mit generell schichtparalleler Orientierung und grüngelber bis gelegentlich rötlichgrüner Fluoreszenz (Anhang 2-12, Fig. 04). Abgesehen von zwei Häufigkeitsmaxima, die mit 1,7 Vol.\% (Probe KS23) bzw. 2,2 Vol.\% (Probe KS33) an der Basis und am Top von MFZ 3d: Aulacoseira-Opal(CT)-Fazies entwickelt sind, ist unstrukturierter Lamalginit mit durchschnittlich 0,3 Vol.\% nur ein untergeordneter Bestandteil der Eckfelder Profundalsedimente. Innerhalb der MFZ 3e: Tetraedron-Opal(CT)-Fazies tritt der Mazeral-Typ nur selten isoliert auf. Insbesondere in tonreichen Sedimentabschnitten sind dagegen häufig laterale Übergänge von unstrukturiertem, teils anastomisierendem Lamalginit zu diffus begrenztem, rötlich fluoreszierendem Bituminit zu beobachten. Auf der anderen Seite gehen unstrukturierte Lamalginite in vielen Proben der MFZ 3e (KS40, KS41, KS43, KS45, KS51, $\mathrm{BL} / \mathrm{TO}(\mathrm{CT})-\mathrm{F} 2.3)$ kontinuierlich in horizontale Massenansammlungen von un- oder nur schwach kompaktierten Tetraedron-Zellen über. Dieser deutliche Übergang von intern unstrukturiertem zu strukturiertem Lamalginit (Tetraedron) ist generell an einen Materialwechsel von stärker tonigen zu opal(CT)-reichen Profilabschnitten gebunden. Er ist eine Folge der unterschiedlich starken Kompaktion beider Sediment-Typen (vgl. Kap. 4.3.5 und Kap. 6.5.3). Ähnliches ist auch aus den mitteleozänen Seeablagerungen von Messel belegt. JANKOWSKI \& LITTKE (1986) beschreiben gelblich fluoreszierende, intern homogene Liptinitlagen und -fetzen, die mit zunehmender Länge und/oder Konzentration zu einer feinen Lamination der Sedimente beitragen. "Da man oft laterale und vertikale Übergänge von intern unstrukturierten zu schwach strukturierten Lagen und weiter zu individuell erkennbaren Algenkörpern beobachten kann, werden für diese Lagen die coccalen Algen als Ausgangsmaterial angenommen." (JANKOWSKI \& 
LITTKE 1986, S. 78.) Die gelb bis rötlichgelb fluoreszierenden coccalen Algen wurden später von GoTH (1990) als Tetraedron minimum identifiziert.

\subsubsection{Bituminit}

Tab. 28: Position von Bituminit innerhalb der Mazeralklassifikation

\begin{tabular}{|c|c|c|c|}
\hline Mazeral-Gruppe & Mazeral-Subgruppe & Mazeral & Mazeral-Typ \\
\hline Liptinit & & Bituminit & $\begin{array}{c}\text { Bituminit (gelb fluoreszierend) } \\
\text { Bituminit (rot fluoreszierend) }\end{array}$ \\
\hline
\end{tabular}

Das Liptinit-Mazeral Bituminit wurde von TEICHMÜLLER (1974) eingeführt. Im Gegensatz zu anderen Mazeralen zeigt Bituminit keine charakteristische Form oder Internstruktur, die einen direkten Hinweis auf seine Bildungsbedingungen liefern könnten (COOK \& KANTSLER 1982, TAYLOR et al. 1998). Das Mazeral tritt jedoch häufig in Ölschiefern auf und ist durch einen vergleichsweise hohen Wasserstoff-Gehalt gekennzeichnet (TEICHMÜLLER 1989). Sehr wahrscheinlich repräsentiert Bituminit die bakteriellen Abbauprodukte von Algen und Zooplankton zusammen mit einem hohen Anteil an bakterieller Biomasse (SHERWOOD 1984, TAYLOR et al. 1998). Vermutlich sind aber auch huminitische Substanzen an der Zusammensetzung von Bituminit beteiligt (COOK \& KANTSLER 1982, TAULBEE et al. 1990).

Innerhalb der Eckfelder Seesedimente tritt Bituminit in zwei verschiedenen Typen auf, die sich vorwiegend in ihrer Fluoreszenzeigenschaft unterscheiden.

Bituminit (gelb fluoreszierend). Dieser Typ des Bituminits erscheint in unregelmäßig begrenzten Linsen von $100 \mu \mathrm{m}$ bis $200 \mu \mathrm{m}$ Länge und einer Stärke von maximal $25 \mu \mathrm{m}$. Trotz einer ähnlichen Fluoreszenzfarbe unterscheidet sich gelb fluoreszierender Bituminit durch ein sehr viel geringeres Längen:Breiten-Verhältnis von unstrukturiertem Lamalginit (vgl. Kap. 6.4.1). Das charakteristische Merkmal dieses Bituminit-Typs ist jedoch eine ungleichmäßige aber deutliche "Schlieren"-Textur in Verbindung mit einem häufigen Verblassen der Fluoreszenzintensität zu den Rändern hin (Anhang 2-12, Fig. 06).

Gelb fluoreszierender Bituminit ist mit durchschnittlich 0,3 Vol.\% am Sedimentaufbau beteiligt, zeigt aber über die gesamte Abfolge der Lithofazieszonen 2: Übergangsschichten und 3: Biogene Laminite hinweg sporadische Häufigkeitsmaxima bis 1,8 Vol.\% (Probe KS33).

Bituminit (rot fluoreszierend). Im Gegensatz zu der oben genannten Form des Bituminits ist rötlich fluoreszierender Bituminit lediglich in der MFZ 3e: Tetraedron-Opal(CT)-Fazies mit nennenswerter Häufigkeit vertreten. Hier heben sich mit 1,7 Vol.\% (Probe KS41) und 0,8 Vol.\% (Probe KS44) zwei deutliche Maxima ab, die jeweils mit einem markanten Anstieg der Tetraedron-Werte zusammenfallen. Rot fluoreszierender Bituminit ist durch eine vergleichsweise geringe Fluoreszenzintensität und eine hochvariable Form gekennzeichnet. In den tonreichen Horizonten der MFZ $3 e$ bildet er oft die laterale Erweiterung von unstrukturiertem Lamalginit, der seinerseits klar mit dem Vorkommen von Tetraedron-Zellen assoziiert ist (vgl. Kap. 6.4.1). Häufig geht rot fluoreszierender Bituminit auch direkt aus Tetraedron-reichen Opallagen hervor. 


\subsection{Authigene Minerale}

\subsubsection{Pyrit}

Bis auf eine Ausnahme (Probe KS13) ist Pyrit in sämtlichen Proben der zentrumsnahen Bohrungen E1/96 und E2/96 mit Werten von üblicherweise $0,1 \mathrm{Vol}$ \% bis 3,3 Vol.\% nachzuweisen. Einen Sonderfall bildet die basale Mikrofazieszone der Lithofazieszone 2: Übergangsschichten. Hier treten Fe-Sulfide mit über 85 Vol.\% (Probe KS2) bzw. rund 48 Vol.\% (Probe KS3) gesteinsbildend auf. Der extreme Pyritreichtum in MFZ 2a: Quarz-Pyrit-Fazies korreliert bemerkenswerter Weise mit dem stratigraphisch ersten Nachweis partikulärer organischer Substanz - vorwiegend pflanzlicher Detritus (Huminit) und Botryococcus - in nennenswerten Quantitäten. In diesem Profilbereich bildet das Fe-Mineral großflächige Massenansammlungen von xeno- bis hypidiomorphen Einzelkristallen mit einem Durchmesser von rund $1 \mu \mathrm{m}$. Gelegentlich umschließen die strukturlosen Pyritmassen sphärische Pyritframboide oder gehen in schichtparallel gelängte Framboid-Aggregate (Polyframboide) über (Anhang 2-1, Fig. 07 und Fig. 08).

Im Hangenden von MFZ 2a ist Pyrit nahezu ausschließlich in Form von isolierten, $5 \mu \mathrm{m}$ bis $30 \mu \mathrm{m}$ großen Framboiden vertreten, die ihrerseits aus $1 \mu \mathrm{m}$ bis $2 \mu \mathrm{m}$ großen, pentagondodekaedrischen und oktaedrischen Einzelkristalliten aufgebaut sind (Anhang 2-12, Fig. 07). Auffällig ist eine generelle Konzentration von Framboiden in ton- und organisch-reichen Horizonten über den gesamten Abschnitt der untersuchten Sedimentfolge hinweg. In seltenen Fällen wurden innerhalb der Lithofazieszone 3: Biogene Laminite längliche Aggregate aus lediglich drei bis fünf idiomorphen Pyrit-Oktaedern beobachtet, die mit einem Durchmesser von knapp

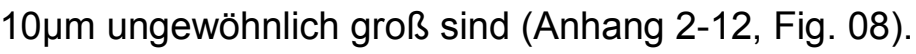

Pyrit entsteht unter anoxischen Bedingungen aus der Reaktion von gelöstem Schwefelwasserstoff $\left(\mathrm{H}_{2} \mathrm{~S}, \mathrm{HS}^{-}\right)$mit detritischen Eisenmineralen (Abb. 22). Das für die Pyritgenese notwendige $\mathrm{H}_{2} \mathrm{~S}$ ist ein Endprodukt der bakteriellen Sulfatreduktion unter der Beteiligung von organischer Substanz als Reduktionsmittel und Energiequelle (BERNER 1971, BERNER 1984). Nach SHANKS et al. (1976) und WESTRICH \& BERNER (1984) läßt sich der komplexe Vorgang der mikrobiellen Sulfatreduktion durch die vereinfachte Reaktionsgleichung

$$
2 \mathrm{CH}_{2} \mathrm{O}[\mathrm{SOM}]+\mathrm{SO}_{4}^{2-} \rightarrow \mathrm{H}_{2} \mathrm{~S}+2 \mathrm{HCO}_{3}^{-}
$$

beschreiben. Die Menge des freigesetzten Schwefelwasserstoffs ist also in direktem Maße abhängig von der Verfügbarkeit sowohl an gelöstem Sulfat als auch an degradierbarer organischer Substanz. In lakustrinen Milieus liegt die Sulfatreduktionszone meist in den obersten Dezimetern des Sediments (HANSELMANN 1989, KATZ 1990). Im Vergleich zu Meerwasser (30 $\mathrm{mmol} / \mathrm{l}$ ) ist die Sulfat-Konzentration in Seen mit 0,05-0,3 $\mathrm{mmol} / \mathrm{l}$ jedoch üblicherweise gering und somit einer der limitierenden Faktoren für die Sulfatreduktionsaktivität von Bakterien (BERNER 1984, DAVISON 1988).

Aus der Reaktion von dissoziiertem Schwefelwasserstoff mit mineralisch gebundenem Eisen entstehen zunächst amorphe Fe-Sulfide nach der Formel

$\mathrm{Fe}^{2+}+\mathrm{HS}^{-} \rightarrow \mathrm{FeS}+\mathrm{H}^{+}$

(DAVISON 1988). 
Am reaktivsten hierfür sind feinkörnige Eisenoxide und -hydroxide, die als Verwitterungsbelag anderen Mineralkörnern ansitzen oder an organische Substanzen gebunden sind (BERNER 1971, 1981b, 1984). Als weitere Eisenquelle kommen unverwitterte Primärminerale, wie Biotit, Pyroxen, Amphibol und eisenhaltige Tonminerale, in Frage. Amorphe Fe-Sulfide altern in energieärmere, höher kristalline und weniger lösliche Übergangsprodukte [Mackinawit (FeS) und Greigit $\left(\mathrm{Fe}_{3} \mathrm{~S}_{4}\right)$ ] (BERNER 1971, 1981b; DAVISON 1988). Die Umwandlung von metastabilen Eisensulfiden zu Pyrit erfordert jedoch einen vermutlich mikrobiell induzierten, oxidativen Elektronentransfer nach der Formel
$\mathrm{FeS}+\mathrm{S}^{0} \rightarrow \mathrm{FeS}_{2}$
bzw.
$\mathrm{Fe}_{3} \mathrm{~S}_{4}+2 \mathrm{~S}^{0} \rightarrow 3 \mathrm{FeS}_{2}$
(BERNER 1971).

Der Verlauf der oxidativen Reaktion von elementarem Schwefel und Eisenmonosulfiden zu Pyrit ist im Detail wenig verstanden (DAVISON 1988, WIGNALL 1994). Allerdings scheinen hierbei Zwischenstufen der Schwefeloxidation, wie beispielsweise Thiosulfat $\left(\mathrm{S}_{2} \mathrm{O}_{3}{ }^{2-}\right)$, maßgeblich beteiligt zu sein (JØRGENSEN 1990). In Ablagerungsräumen mit anoxischen Bodenwasser, wie meromiktische Seen oder euxinische Meeresbecken, kann es bereits innerhalb der Wassersäule zu einer Reaktion von gelöstem $\mathrm{H}_{2} \mathrm{~S}$ mit detritischen Eisenmineralen kommen. Die Sulfidbildung findet in solchen Fällen direkt an oder sogar oberhalb der Sediment/Wassergrenze statt (BERNER 1971, 1984; DAVISON 1988).

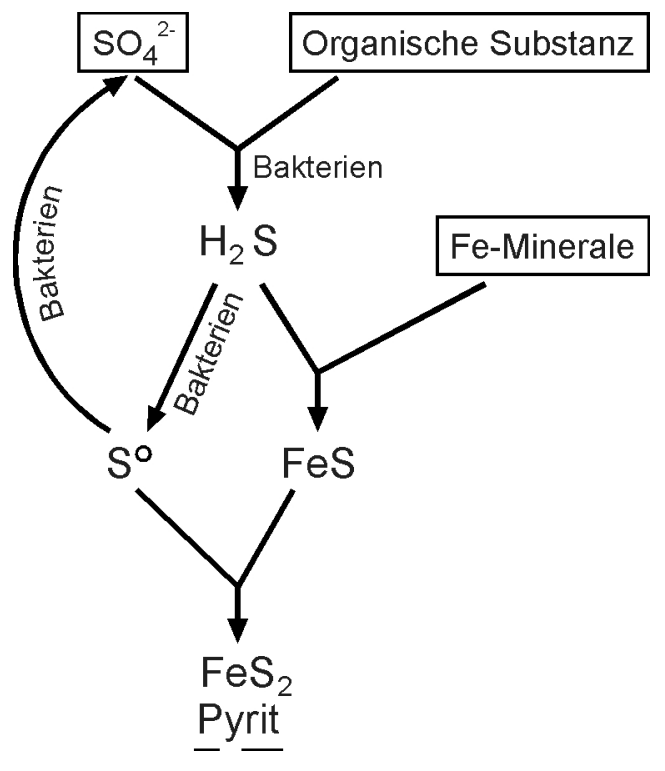

Abb. 22: Die Genese von Pyrit (nach BERNER 1981).

Die Genese von Pyrit in seiner framboidalen Form unterliegt immer noch der Diskussion. SCHNEIDERHÖHN (1923) interpretiert Pyritframboide als mineralisierte Bakterien. Dieser These folgen auch spätere Autoren (NeuHaus 1940, Love 1957, Love 1962a, b; Love \& ZIMMERMAN 1961, FABRICIUS 1962, FOSTER et al. 1985). BERNER $(1969,1971)$ und KRIBEK (1975) beschreiben die Entstehung von framboidalem Pyrit hingegen als eine ausschließlich anorganische Reaktion. Am Beispiel der Messeler Seesedimente diskutiert LIEBIG (1998) ausführlich die verschiedenen Theorien zur FramboidBildung. Nach ihrer Auffassung kristallisiert Pyrit anorganisch aus einem Eisensulfid-Gel. Die kolloidale Pyritgenese erlaubt ein freies Kristallwachstum und die Ausbildung idiomorpher Einzelkristallite, wie sie auch für die Framboide in Eckfeld charakteristisch sind. Ihre framboidale Form erhalten die Mikrokonkretionen während des Übergangs von Mackinawit zu Greigit (SWEENEY \& KAPLAN 1973). Pyrit entsteht üblicherweise innerhalb weniger Jahre als Pseudomorphose nach Greigit-Framboiden (CANFIELD \& RAISWELL 1991, WIGNALL 1994). 
Amorphe Eisensulfide sind mobil und können bestehende Hohlräume infiltrieren (LIEBIG 1998). Dieser Umstand erklärt in idealer Weise die vielfach pyritisierten Zellhohlräume von TextoUlminiten innerhalb der MFZ 2a: Quarz-Pyrit-Fazies (vgl. Kap. 4.2.1).

\subsubsection{Siderit}

Nach (PIRRUNG 1998, Abb. 9) ist authigenes Fe-Karbonat in der Bohrung E1/96 ab einer Teufe von rund $33,5 \mathrm{~m}$ regelmäßig nachzuweisen. Diese Profillage korreliert mit dem Basisbereich der Lithofazieszone 3: Biogene Laminite (MFZ 3a: Huminit-Fazies) und ist auch in E3/96 durch das erstmalige Auftreten vorwiegend isolierter Siderit-Linsen im Sediment gekennzeichnet. Der Sideritreichtum eines bei PIRRUNG (1998) verzeichneten Profilbereichs zwischen 38,0m und 39,5m (E1/96) konnte im entsprechenden Detailprofil der Bohrung E3/96 (ML/Q-F1, Anhang 1-1) dagegen nicht bestätigt werden.

In der Lithofazieszone 3 ist der Hauptanteil des Fe-Karbonats in $20 \mu \mathrm{m}$ bis $300 \mu \mathrm{m}$ mächtigen Horizonten und isolierten Linsen konzentriert. Im BSE-Bild zeigen sie sich als überwiegend lockere Ansammlungen von $2 \mu \mathrm{m}$ bis $8 \mu \mathrm{m}$ großen, meist xenomorphen Siderit-Einzelkörnern (Anhang 2-4, Fig. 07). Die sideritreichen Horizonte treten typischerweise innerhalb von organisch-reichen Tonlaminen auf oder ersetzen diese im Wechsel mit opalreichen Lagen (Anhang 2-5, Fig. 06). In der MFZ 3b: Aulacoseira-Fazies sind die Grenzbereiche zwischen den Tonlaminen (LT 3b-2) und den überlagernden Diatomeenlagen (LT 3b-1) oftmals durch 10 $\mu \mathrm{m}$ bis $30 \mu \mathrm{m}$ mächtige Anreicherungen von Siderit in Assoziation mit Pyritframboiden gekennzeichnet (Anhang 2-3, Fig. 03).

Die Bildungsbedingungen von Sideritlaminae in paläogenen Seen Mitteleuropas sind gegenwärtig noch nicht befriedigend geklärt (FELDER et al. 2000a). Während BAHRIG (1989), MingRAM (1994, 1998) und FELDER \& GAUPP (2001) für die Seesedimente von Messel und Eckfeld von einer frühdiagenetischen Bildung ausgehen, diskutieren GOTH (1990) und LUTZ (1993b, 1998, 1999, 2000) die Möglichkeit einer primären Sideritfällung aus der Wassersäule. Eine primäre Fällung von Fe-Karbonat oberhalb der Sediment/Wasser-Grenze sollte zu vergleichsweise reinen und horizontbeständigen Sideritlagen führen. Reine Sideritlagen wurden innerhalb der Lithofazieszone 3 jedoch in keinem Fall beobachtet. Sideritreiche Horizonte beinhalten immer Anteile von Umgebungssediment in Form von Tonlinsen und undeutlich begrenzten, tonreichen Schlieren. Innerhalb der Sedimentsäule von E3/96 ändert sich die Zusammensetzung der organischen Partikel in Sideritlagen von vorwiegend Huminit in MFZ 3a hin zu vorwiegend Alginit in MFZ 3e. Das organische Partikel-Inventar entspricht jedoch immer dem der jeweils angrenzenden Tonlagen. Die häufig linsenförmigen Konzentrationen von Fe-Karbonat in tonreichen Sedimentabschnitten sprechen gegen eine flächenhafte Siderit-Fällung aus der Wassersäule. Vielmehr zeigen die teils signifikanten Verunreinigungen durch Umgebungssediment und die Gleichartigkeit von POM in karbonat- und tonreichen Horizonten eine Sideritgenese innerhalb des Sediments an. In der MFZ 3e: Tetraedron-Opal(CT)-Fazies sind Tetraedron-Zellen als Bestandteil sideritreicher Lagen typischerweise dreidimensional überliefert. Dies kann als deutlicher Hinweis auf eine frühdiagenetische Sideritbildung gewertet werden, die vermutlich nur wenige Zentimeter unterhalb der Sediment/Wassergrenze einsetzte. Während Tetraedron infolge der Sedimentkompaktion in tonreichen Profilabschnitten stark 
verdrückt wurde, blieb die Alge gerade in Sideritlagen vollkörperlich erhalten (siehe auch FELDER et al. 2000b).

Das gemeinsame Vorkommen von Siderit und Pyrit in den organisch-reichen Sedimenten der Lithofazieszone 3 ist nach BERNER (1981a, b) kennzeichnend für ein anoxischnichtsulfidisch-methanisches Milieu. Unter diesen geochemischen Bedingungen sind die Voraussetzungen für eine Sideritbildung erst nach der weitgehenden Konsumierung von $\mathrm{HS}^{-}$ durch die Reaktion mit detritischen Eisenmineralen gegeben (vgl. Kap. 6.5.1). Nach BAHRIG (1989) fällt alles verfügbare Eisen oberhalb einer Konzentration von $10^{-7} \mathrm{~mol} / / \mathrm{HS}^{-}$als Fe-Sulfid aus. In den meisten lakustrinen Milieus werden gelöste Sulfide $\left(\mathrm{H}_{2} \mathrm{~S}\right.$ und $\left.\mathrm{HS}^{-}\right)$im Zuge der Pyritgenese nahezu vollständig verbraucht. Durch eine fortlaufende mikrobielle Eisenreduktion nach der idealisierten Reaktionsgleichung

$\mathrm{CH}_{2} \mathrm{O}[\mathrm{SOM}]+7 \mathrm{CO}_{2}+4 \mathrm{Fe}(\mathrm{OH})_{3} \rightarrow 4 \mathrm{Fe}^{2+}+8 \mathrm{HCO}_{3}^{-}+3 \mathrm{H}_{2} \mathrm{O}$

kann es daher zur Anreicherung von $\mathrm{Fe}^{2+}$ im Porenwasser des Sediments kommen (BERNER 1981b, PostmA 1982). Die geringe, sehr gleichförmige Größe der Sideritkristalle in den Eckfelder Seesedimenten ist ferner ein Indiz für die Fällung aus einer Fe-übersättigten Lösung (FELDER frdl. schriftl. Mitt. 2001). Nach POstMA (1982) müssen Porenwasserlösungen generell an Eisen übersättigt sein, bevor es zu einer Sideritfällung kommen kann. Übereinstimmend belegt EMERSON (1976) die zehnfache Übersättigung in bezug auf Siderit im Porenwasser der Greifensee-Sedimente (Schweiz). Ein reduzierendes Milieu mit Eh-Werten von $<+100 \mathrm{mV}$ bei $\mathrm{pH} 7$ ist sowohl für die thermodynamische Stabilität von Siderit als auch für die Mobilisierung ausreichender Mengen von $\mathrm{Fe}^{2+}$ erforderlich. Als eine weitere Voraussetzung für die Sideritbildung gibt BAHRIG (1989) einen $\mathrm{CO}_{2}$-Partialdruck von $>10^{-6} \mathrm{~atm}$ an. Im Falle von Eckfeld deuten die extrem positiven $\delta^{13} \mathrm{C}$-Werte von Siderit auf die mikrobielle Methanogenese als eine wesentliche $\mathrm{CO}_{2}$-Quelle hin (BAHRIG 1989).

\subsubsection{Opal-CT}

Im Gegensatz zu den überdurchschnittlich gut erhaltenen Aulacoseira-Frusteln der MFZ 3b: Aulacoseira-Fazies sind die diatomeenreichen Lagen der MFZ 3d: Aulacoseira-Opal(CT)-Fazies durch eine fortgeschrittene Umwandlung von Skelettopal (Opal-A) hin zu Opal-CT gekennzeichnet. Diese Phasentransformation ist keine Festkörperreaktion, sondern vollzieht sich über die Auflösung und Wiederausfällung von opaliner Substanz (KEENE 1976, STEIN \& KIRKPATRICK 1976, MURATA et al. 1977, TADA \& IIJIMA 1983). Die allgemein diffusen Schichtgrenzen zwischen kieseligen und tonreichen Horizonten in MFZ 3d, insbesondere aber in der hangenden MFZ 3e: Tetraedron-Opal(CT)-Fazies, belegen zudem eine laterale und vertikale Migration gelöster Kieselsäure (vgl. Kap. 4.3.4 und 4.3.5). Nach TADA (1991) wird der Zeitpunkt der Opal(CT)Bildung durch leichte Unterschiede in der primären Lithologie zweier aufeinanderfolgender Lagen bestimmt. Dadurch ist ein vertikaler Konzentrationsgradient von gelöster Kieselsäure zu erwarten: "The bed in which opal-CT is first precipitated tends to import dissolved silica, whereas silica tends to be exported from the neighboring bed in which opal-CT precipitation is retarded or inhibited [...]" (TADA 1991, S. 487). 
Kieselsäurekonzentration im Porenwasser
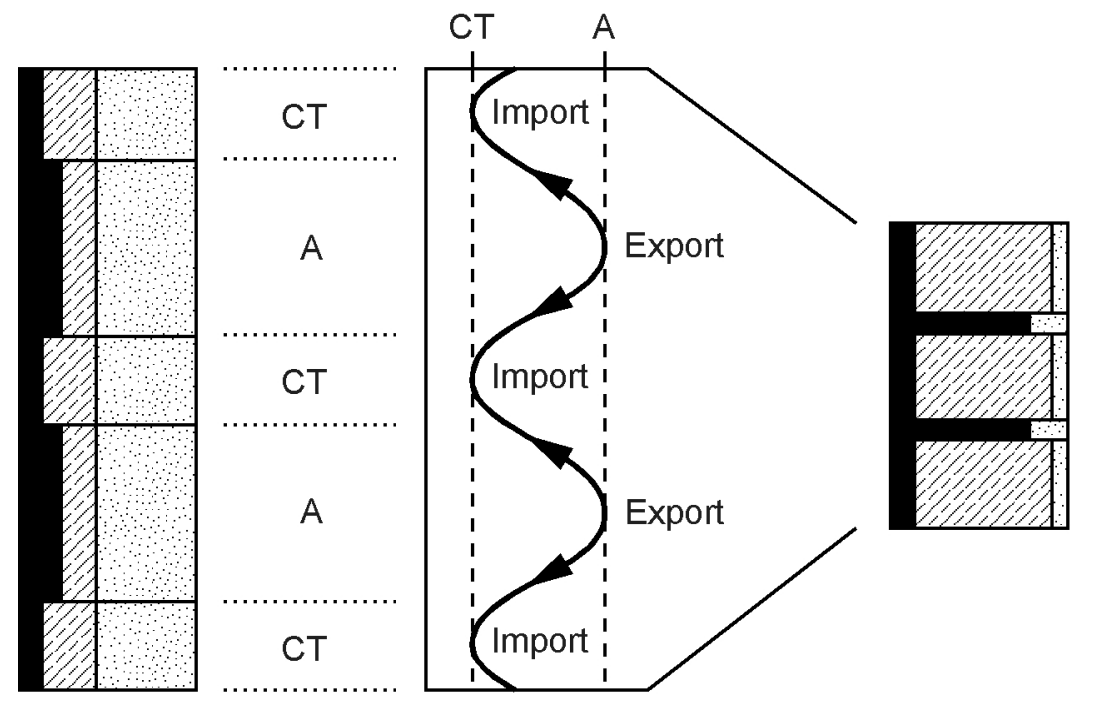

\section{Ton Opal Poren}

Kieselsäure-Migration während der Opal(A) $\rightarrow$ Opal(CT) Transformation

Abb. 23: Mechanismus der diagenetischen Kieselsäure-Umverteilung in Ton/Opal-Wechselfolgen (nach TADA 1991).

Abgesehen von einem ohnehin vergleichsweise geringen Gehalt an kieseligen Komponenten (Diatomeen-Frusteln und Schwamm-Skleren) in den tonreichen Horizonten der Mikrofazieszonen $3 d$ und $3 e$, ist von vielen Autoren eine verzögerte Opal(A)-Opal(CT)-Transformation in tonhaltigen Sedimenten beobachtet worden (KASTNER et al. 1977, ISAACS 1981, FÜCHTBAUER 1988a). In Ton/Opal-Wechselfolgen führt die diagenetische $\mathrm{SiO}_{2}$-Umverteilung von opalarmen zu opalreichen Schichten nach DECKER (1991) zu einer abnehmenden Mächtigkeit von Tonlagen. Für eine beginnende Opal(CT)-Bildung in den ehemals diatomeenreichen Schichten in Verbindung mit einer kontinuierlichen Mächtigkeitsabnahme toniger Horizonte durch den Export von Kieselsäure sprechen in Eckfeld eine Reihe von Beobachtungen:

- Die Amphioxe von Schwämmen sind vorwiegend in den tonreichen Horizonten der Mikrofazieszone 3d: Aulacoseira-Opal(CT)-Fazies erhalten geblieben. Innerhalb der opal(CT)-reichen Lagen kommen sie dagegen nur noch selten und lediglich nahe der Schichtgrenzen vor. Die Opaldiagenese ist also offenbar in den kieseligen Horizonten weiter fortgeschritten.

- $\quad$ Der Lösungsgrad von Diatomeen-Schalen nimmt von MFZ 3d: Aulacoseira-Opal(CT)Fazies nach MFZ 3e: Tetraedron-Opal(CT)-Fazies kontinuierlich zu. Damit geht eine fortlaufende Mächtigkeitsabnahme von unscharf begrenzten Tonlagen relativ zu Opal(CT)-reichen Horizonten einher. Im oberen Abschnitt von MFZ 3e sind primäre Schichtungsgefüge nahezu vollständig überprägt. Ehemals tonreiche Schichten lassen sich im BSE-Bild oftmals nur noch durch die lagenweise Anreicherung von organischen Partikeln nachvollziehen.

- Innerhalb der diagenetisch unbeeinflußten MFZ 3b: Aulacoseira-Fazies sind Huminite und Liptinite typische Bestandteile tonreicher Schichten. In MFZ 3d treten sie dagegen auch in den unscharf begrenzten Randbereichen von Opallagen konzentriert auf. Dieses ist ein 
deutlicher Hinweis auf eine Verschiebung der ursprünglichen Schichtgrenzen in die ehemaligen Tonlagen hinein.

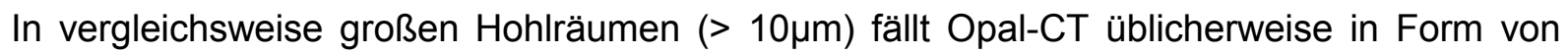
sphärischen Aggregaten (Lepisphären) aus (WISE \& KELTS 1972, GOTH 1990, LIEBIG 1998). Die Lepisphären bestehen aus diskreten Tafeln von Tieftemperatur $\alpha$-Cristobalit, der durch zwischengelagerten Tridymit eindimensional fehlgeordnet ist. Innerhalb der sphärischen Struktur sind die Opal(CT)-Tafeln nach dem Zwillingsgesetz von Tridymit in einem Winkel $70^{\circ}$ bis $71^{\circ}$ miteinander verwachsen (OEHLER 1975, FLÖRKE et al. 1976, RAD 1979, LIEBIG 1998).

Die maximal $3 \mu \mathrm{m}$ großen Lepisphären treten in MFZ 3d: Aulacoseira-Opal(CT)-Fazies und MFZ 3e: Tetraedron-Opal(CT)-Fazies vorwiegend in den Hohlräumen bzw. Abdrücken von Diatomeen-Schalen auf und lassen sich rasterelektronenmikroskopisch zweifelsfrei nachweisen (Anhang 2-6, Fig. 04). Demgegenüber sind die Hohlformen gelöster Kieselskelette in den obersten $5 \mathrm{~m}$ von MFT $3 e$ weitgehend frei von Lepisphären (Anhang 2-6, Fig. 05). Statt dessen finden sich Massenansammlungen kugeliger Opal(CT)-Aggregate, die häufig auch gesteinsbildend sind (Anhang 2-6, Fig. 06).

Die Transformation von Skelettopal zu Opal-CT ist in den Eckfelder Profundalsedimenten typischerweise durch eine Aufnahme von Al in die Opalsubstanz gekennzeichnet. Während der mineralische Anteil diagenetisch unveränderter Diatomeen-Frusteln in MFZ 3b: AulacoseiraFazies aus weitgehend reinem $\mathrm{SiO}_{2}$ besteht, zeigen EDX-Analysen an den Opal(CT)-Lepisphären höherer Profillagen ein Si/AL-Verhältnis von etwa 3:1 (Anhang 2-13, Fig. 02). Ähnliches weist auch SCHILLER (2000) an kieseligen Mikrofossilien aus den oberoligozänen Seesedimenten von Enspel/Westerwald nach. In der Kernbohrung Enspel 1991(2) wurde Opal im Druckschatten von Diatomeen-Schalen und Goldalgen-Zysten in Form von Steinkernen ausgefällt. Die EDX-Analyse dieser Hohlraumfüllungen zeigte neben $\mathrm{Si}$ auch Gehalte an $\mathrm{Al}$ und $\mathrm{Fe}$. In geringerem Maße wurden $\mathrm{Mg}, \mathrm{Ca}, \mathrm{K}$ und $\mathrm{Ti}$ in die Opalsubstanz aufgenommen.

Die Phasentransformation von Opal-A zu Opal-CT wird durch die Faktoren Zeit, Temperatur, Druck und Porenwasserchemie beeinflußt (HEIN et al. 1978, IIJIMA \& TADA 1981, TADA 1991). Aufgrund der nur geringen diagenetischen Überprägung von organischer Substanz (vgl. Kap.4.3) können erhöhte Druck- und Temperaturbedingungen für die Eckfelder Seesedimente ausgeschlossen werden. Zudem ist kaum anzunehmen das die exzellent erhaltenen Diatomeen-Frusteln der MFZ $3 \mathrm{~b}$ anderen physikalischen Bedingungen ausgesetzt waren, als die im Abstand von 2,5 Bohrmetern folgenden Sedimente der opal(CT)reichen Mikrofazieszonen 3d und 3 e. Auch der Zeitfaktor kann für die Opaldiagenese nicht ausschlaggebend gewesen sein, da gerade die älteren Sedimente durch unveränderten Skelettopal gekennzeichnet sind.

In den opal(CT)reichen Horizonten der MFZ 3e sind die dünnwandigen Einzelzellen von Tetraedron vielfach unverdrückt überliefert (vgl. Kap. 4.3.5 und Kap. 6.4.1). Dieses deutet klar auf eine äußerst frühdiagenetische Imprägnierung des Sediments durch die Ausfällung gelöster Kieselsäure hin. Die Umwandlung von Skelettopal zu Opal-CT vollzog sich in Teilbereichen des Profils offensichtlich vor einer weitreichenden Sedimentkonsolidierung. Eine dreidimensionale Überlieferung von Tetraedron wäre andernfalls kaum zu erklären. Allem Anschein nach war der Chemismus, insbesondere der $\mathrm{pH}$-Wert des Porenwassers maßgeblich für den Erhaltungsgrad kieseliger Mikrofossilien in Eckfeld ausschlaggebend. Es ist von einer beginnenden Lösung von 
Skelettopal bereits wenige Zentimeter unterhalb der ehemaligen Sediment/Wasser-Grenze auszugehen. Zu der Vorstellung einer frühdiagenetischen Opaldiagenese kommt auch MöRS (1995) am Beispiel des lakustrinen Oberoligozäns von Rott/Siebengebirge. Im Gegensatz dazu nimmt GотH (1990, S. 22) aufgrund des Vorkommens wenig kompaktierter Schalenabdrücke in den mitteleozänen Seeablagerungen von Messel eine "relativ spätdiagenetische" Lösung von Diatomeen-Gehäusen erst nach der Sedimentsetzung an. Unkompaktierte Hohlformen gelöster Diatomeen-Frusteln wurden auch in den opal(CT)reichen Profilabschnitten der MFZ 3e: Tetraedron-Opal(CT)-Fazies nachgewiesen. Ihr Auftreten widerspricht einer frühdiagenetischen Lösung von Skelettopal jedoch nicht, da gerade die frühzeitige Imprägnierung durch Kieselsäure zu einer Verfestigung des Sediments führt und seine Kompaktierbarkeit im Vergleich zu Tonlagen herabsetzt. Dieses kommt in dem kontinuierlichen Übergang von unverdrückten zu verdrückten Tetraedron-Exemplaren bzw. zu unstrukturiertem Lamalginit zum Ausdruck, dort, wo ein Materialwechsel von opal(CT)reichen zu tonreichen Horizonten zu beobachten ist (vgl. Kap. 6.4.1). Daß die lösungsbedingte Abnahme von Diatomeen schon in den obersten 1,5 Sedimentzentimetern (entsprechend einem Ablagerungszeitraum von zehn Jahren) stattfinden kann, ist rezent aus dem Michigan-See bekannt und hat hier einen rapiden Anstieg der Kieselsäurekonzentration im Porenwasser zur Folge (PARKER \& EDGINGTON 1976).

Innerhalb der MFZ 3b: Aulacoseira-Fazies weisen die Basisbereiche von Diatomeenlagen oftmals erhöhte Konzentrationen an Siderit und Pyritframboiden auf. Die Aulacoseira-Schalen sind hier vielfach durch eine Anlagerung eisenhaltiger Mineralphasen gekennzeichnet und werden im BSE-Bild deutlich heller dargestellt als unveränderter Skelettopal. Eisenreiche Gehäusereste lassen sich auch in höheren Profillagen (MFZ 3d: Aulacoseira-Opal(CT)-Fazies und MFZ 3e: Tetraedron-Opal(CT)-Fazies) nachweisen und zeigen gegenüber eisenfreien Kieselschalen ein allgemein höheres Erhaltungspotential. Den Einfluß adsorbierter Metall-Ionen auf die Opaldiagenese zeigen Lösungsexperimente an fossilen und rezenten DiatomeenSchalen (LEWIN 1961, BARKER 1992). Beide Autoren konnten übereinstimmend wahrscheinlich machen, daß eine Anlagerung insbesondere von Eisen und Aluminium die Löslichkeit von Skelettopal merklich herabsetzt.

\subsubsection{Apatit}

In den Bohrungen E1/96 und E3/96 schließt die MFZ 3b: Aulacoseira-Fazies mit einem rund 2,65m mächtigen Rutschkörper (Typ-III-Resediment, Kap. 5.1.3) ab, der in ähnlicher Mächtigkeit ebenfalls aus der Bohrung von 1980 (Teufe: 44,5m bis 42,5m) bekannt ist. Das Konglomerat aus intern gefalteten Diatomeen-Laminiten ist in seinem oberen Teil durch Apatit stark verfestigt (NEGENDANK et al. 1982). Im Bohrprofil von E3/96 wurde Ca-Phosphat unmittelbar im Hangenden des Resediments innerhalb der MFZ 3c: Huminit-Opal-Fazies nachgewiesen (Probe BL/HO-F1.1). Aus den untersuchten Proben der liegenden Mikrofazieszonen ist Apatit dagegen nicht belegt. In BL/HO-F1.1 (MFZ 3c) und BL/AO(CT)-F1.8 (MFZ 3d) sitzen unregelmäßige Aggregate aus $2 \mu \mathrm{m}$ bis $8 \mu \mathrm{m}$ großen Phosphat-Körnern jeweils einem ovalen Partikel

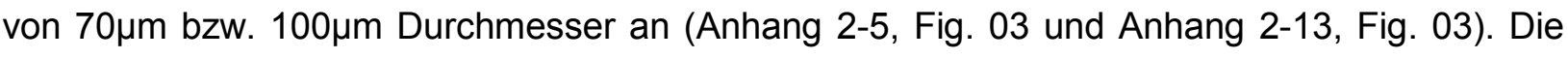
mit ihrer Längsachse schichtparallel orientierten Partikel erscheinen im BSE-Bild dunkelgrau bis schwarz und bestehen nach EDX-Analysen überwiegend aus Kohlenstoff und Sauerstoff. Es handelt sich somit um organisches Material, das aufgrund der Mineralumkrustungen aber nicht 
näher identifiziert werden konnte. Bei den feinkörnigen Apatiten ist Fluor das Anion 2. Stellung. Zum einem geringen Teil werden $\mathrm{Ca}$ durch $\mathrm{Na}$ bzw. Mg und die $\mathrm{PO}_{4}$-Gruppe durch Karbonat vertreten. EDX-analytisch sind die Mineralkörner somit als Karbonatfluorapatit (Francolith) anzusprechen (Anhang 2-13, Fig. 03). Nach verschiedenen Autoren (vgl. LIEBIG 1998) ist das Auftreten von Francolith ein Hinweis auf die Beteiligung phosphatisierter Bakterien an der Weichteilerhaltung von Fossilien. Die innerhalb des Apatit-Aggregats differenzierbaren Einzelkörner erscheinen jedoch insgesamt zu groß für Bakteriomorphen. Allerdings verhindert die ausgeprägte Weiß-Überstrahlung eine feinmorphologische Analyse der Phosphat-Körner im BSE-Bild. Eine mikrobiell induzierte Apatitgenese kann daher nicht mit Sicherheit ausgeschlossen werden.

Bei 21,9m durchteufte die Bohrung E3/96 eine $6 \mathrm{~cm}$ mächtige Ca-Phosphat-Konkretion, die den gesamten Kerndurchmesser einnimmt (Anhang 1-5). Da in den analysierten Proben (BL/AO(CT)-F1.5 und $\mathrm{BL} / \mathrm{AO}(\mathrm{CT})-\mathrm{F} 1.6)$ weder $\mathrm{F}$ noch $\mathrm{Cl}$ detektiert wurden, kann das in derbkörnigen Massen auftretende Ca-Phosphat als Hydroxylapatit angesprochen werden. Auch hier ist Ca partiell durch Mg substituiert. Die teils vollständig eingeschlossenen Laminit-Fragmente in den Randbereichen der Konkretion belegen eine intraformationelle Apatitausscheidung. Dabei wurde das unmittelbar angrenzende Schichtungsgefüge weitgehend zerstört. Auffällig ist, daß die Laminit-Bruchstücke innerhalb der Phosphat-Knolle denselben $\mathrm{SiO}_{2}$-Diagenesegrad aufweisen wie die diatomeenführenden Opal(CT)-Lagen (LT 3d-1) des Umgebungssediments. Ganz offensichtlich bildete sich die Konkretion erst zum Zeitpunkt einer bereits fortgeschrittenen Umwandlung von biogenem Opal-A zu Opal-CT. Neben der dreidimensionalen Erhaltung von Tetraedron-Einzelzellen in den Opal(CT)-Lagen von MFZ 3e ist dies ein weiterer Beleg für eine sehr frühdiagenetische Lösung von Skelettopal innerhalb der beckenzentralen Seesedimente.

In den lakustrinen Ablagerungen von Rott/Siebengebirge liegt Ca-Phosphat ebenfalls in Form von Hydroxylapatit vor und ist, wie auch in Messel, vorwiegend an Koprolithen und Knochenreste gebunden (WUTTKE 1988, MÖRS 1995). Aufgrund seines vergleichsweise großen Durchmessers ist Letzteres auch für den Phosphorit innerhalb der MFZ 3d anzunehmen. Für tierische Hartteile als Phosphor-Quelle spricht ebenfalls das Auftreten einer weiteren ApatitKonkretion in einem Typ-III-Turbidit (vgl. Kap. 5.1.3) bei Bohrmeter 21,2 (E3/96). Aus den Sedimentsequenzen des Eckfelder Grabungsareals ist bekannt, daß gerade die Turbidite eine Vielzahl isolierter Knochen von terrestrischen und amphibischen Vertebraten führen (LUTZ 1993a, DANNAT 2000).

Innerhalb der Augenhöhlen eines 1983 aus dem Grabungsprofil geborgenen PercoidenSkelett wiesen MICKLICH \& WUTTKE (1988) rund $1 \mu \mathrm{m}$ lange Bakteriomorphen nach, die nach EDX-Analysen ausschließlich aus Ca-Phosphat (Hydroxylapatit) bestehen. Die ellipsoiden bis zylindrischen Sphärolithe zeichnen auch die Strukturen ehemaliger Weichteile im Bereich der Dorsalflosse nach und sind wahrscheinlich das Ergebnis einer bakteriell induzierten Apatitfällung. Von Bedeutung war hier vermutlich die Transformation von organisch gebundenem Phosphat, das im Zuge des anaeroben mikrobiellen Stoffwechsels wieder freigesetzt und in anorganischer Form in das Außenmilieu abgegeben wurde. Als Voraussetzung für die Lithifizierung der Barsch-Weichteile geben MICKLICH \& WUTTKE (1988) negative Eh-Werte am Ort der Ablagerung sowie ein pH-Wert zwischen 7 und 9 an. Dieser sank auch im Verlauf der Diagenese nicht unter $\mathrm{pH} 7$. 


\section{Diskussion}

\subsection{Beckengenese und Sedimentationsgeschichte des Eckfelder Maares}

\section{Die frühe Seephase}

Maare entstehen aus dem unterirdischen, hochexplosiven Kontakt von Grundwasser mit einer aufsteigenden Gesteinsschmelze (LORENZ 1987, BÜCHEL 1993). Nach einer initialen, mechanischen Durchmischung beider Phasen (DELLINO 2000), führt die Überhitzung des Wassers zu einer explosiven Ausdehnung von Wasserdampf. Der plötzliche Drucktransfer in angrenzende, wassergefüllte Porenräume hat eine Fragmentierung des Umgebungsgesteins und den Ausstoß von Magma-Fetzen, Wasser und Nebengesteinsfragmenten aus einer schmalen Eruptionsröhre zur Folge (LORENZ 2000). Dieser Prozeß wiederholt sich episodisch (vermutlich zyklisch) solange bis der Magmaaufstieg unterbrochen wird oder die Verfügbarkeit von Grundwasser nachläßt.

Im Falle des Eckfelder Maars fragmentierten phreatomagmatische Eruptionen eine Gesteinssäule aus marinen Sedimenten des Unterems (Unterdevon), denen eine mesozoischtertiäre Verwitterungsdecke unbekannter Mächtigkeit auflagerte (FISCHER 1999). Anhand der für rezente Maarkrater charakteristischen Durchmesser:Tiefe-Relation von 5:1 und einer bei 470m ü. NN rekonstruierten mitteleozänen Landoberfläche kalkulieren PIRRUNG (1998) und PIRRUNG et al. (2001) einen initialen Kraterdurchmesser von etwa 900m. Daraus ergibt sich eine Kratertiefe von rund $170 \mathrm{~m}$.

Die steilen Kraterwände machen Maare zu instabilen morphologischen Gebilden, die bereits während oder unmittelbar nach der eruptiven Phase durch gravitativ gesteuerte Massenverlagerungen gekennzeichnet sind (BÜCHEL 1993). Das beckenzentrale, rund 123m mächtige Bohrprofil von E1/95 erbrachte in seinen untersten 50m matrixfreie, devonklastenreiche Brekzien. Sie werden von PIRRUNG (1998) und FISCHER (1999) als subaerische Fall- und Trümmerstromablagerungen gedeutet. Diesen, an der Basis vermutlich noch syneruptiv abgelagerten, Pyroklastika liegt ab Bohrmeter 71,19m eine rund 30m mächtige Abfolge aus nebengesteinsreichen Brekzien mit zwischengeschalteten, von Kies nach Pelit gradierten Lagen auf (FISCHER 1999). Das gelegentliche Auftreten von laminierten Pelitintraklasten innerhalb der Brekzien und die maximal $1 \mathrm{~m}$ mächtigen, normalgradierten Zwischenlagen belegen die subaquatische Sedimentation in einem Maarsee. Allerdings bestehen nur etwa $10 \%$ des Profilbereichs zwischen 71,19m und 41,35m (Bohrung E1/96) aus gradierten Schichten (FISCHER 1999). Für den Großteil der grobklastischen Abfolge sind subaquatische Ablagerungsbedingungen nicht gesichert. Es ist anzunehmen, daß der Maarsee in seinem frühen Stadium noch verhältnismäßig flach gewesen ist (PIRRUNG 1998) und wiederholt durch subaerische Schuttströme teilweise oder vollständig verfüllt wurde. Im Wechsel zwischen grobklastischen Schüttungsereignissen und dem kontinuierlich ansteigenden Wasserspiegel entstand ein Profilabschnitt aus ungradierten Brekzien und normalgradierten Einschaltungen, für den nach FISCHER (1999) ein Ablagerungszeitraum von mehreren Wochen bis Monaten wahrscheinlich ist. 


\section{Lithofazieszone 1: Minerogene Laminite}

Ein herausragendes Merkmal der mineralischen Ablagerungen an der Basis der untersuchten Sedimentabfolge (MFZ 1a: Quarz-Fazies) ist der mit 0,1 Vol.\% äußerst seltene Nachweis organischer Partikel (vgl. Kap. 4.1.1 und Anlage 2). Dieser Umstand spricht klar für die Vorstellung eines weitgehend unbewachsenen Tephrawalls, der den See in seiner Frühphase umgab und inn von einem organischen Detrituseintrag aus dem Umland isolierte (BULLWINKEL \& RIEGEL 2001). Insbesondere die relative Armut an Sporinit in den minerogenen Laminiten der Lithozone 1 deutet auf eine wirkungsvolle Barriere gegen den Eintrag allochthoner, windgetragener Pollen.

Mit 70\% der Gesamtmächtigkeit machen Grobklastische Resedimente vom Typ I (vgl. Kap. 5.1.1) den Hauptanteil der mineralischen Sedimentfolge aus. Sie lassen sich von "hyperconcentrated density flows" ( $\mathrm{X}_{1}$-Abschnitte) bzw. "concentrated density flows" ( $\mathrm{Y}_{1}$-Abschnitte) ableiten, die distal in geringer konzentrierte "turbidity currents" (Z-Abschnitte) übergingen. Wenige millimeter- bis zentimetermächtige Turbidite sind maßgeblich an der eben parallelen Feinschichtung in diesem Profilabschnitt beteiligt (Anhang 2-7, Fig. 04). Die Häufigkeit von Resedimentationsereignissen belegt eine hohe Reliefenergie entlang der steilen, instabilen Kraterinnenwände. Den siliziklastischen Ereignislagen zwischengelagert sind teils laminierte (Anhang 2-1, Fig. 05 und Fig. 06), teils homogene (Anhang 2-1, Fig. 01 und Fig. 02), feinsandige Pelite (LT 1a-1, LT 1a-2 und LT 1a-3, vgl. Kap. 4.1.1). Sie zeigen keinerlei texturelle oder petrographische Merkmale eines bodennahen Sedimenttransports und können als Ablagerungen aus einer feinkörnigen Sedimentsuspension gedeutet werden. Wechsellagerungen aus grobkörnigen Ereignislagen und pelitischen Sedimentniederschlägen sind von einer ganzen Reihe vulkaniklastisch geprägter Seeablagerungen bekannt (PEDERSEN et al. 1998, RIGGS et al. 2001, PALMER \& SHAWKEY 2001, GAYLORD et al. 2001). Nach NELSON (1967) und STURM (1979) sind unstrukturierte, gering sortierte Pelite (LT 1a-1) in oligotrophen Seen kennzeichnend für eine weitgehend kontinuierliche Zufuhr von Feindetritus. Für den Eckfeld-See ist die Vorstellung eines anhaltenden Eintrags von Suspensionsmaterial angesichts der Nähe eines unbewachsenen Tephrarings als Liefergebiet für feine Korngrößen schlüssig. Obwohl die homogenen Sedimentabschnitte nicht signifikant mit klastenführenden Ereignislagen assoziiert sind, ist davon auszugehen, daß die hochfrequenten Massenverlagerungen beachtliche Mengen von Feinmaterial in den See einbrachten und immer wieder resuspendierten. Bei stärkeren Regenfällen führte die Auswaschung des Kraterrandes zu einem zusätzlichen Eintrag von mineralischem Detritus (PIRRUNG 1998).

Abgesehen von der Tonfraktion bilden silt- und feinsandgroße Quarzkörner die Hauptkomponente der pelitischen Seesedimente (Anhang 2-1, Fig. 01 und Fig. 02 bzw. Fig. 05 und Fig. 06). Sie entstammen zweifelsfrei den bei der Maareruption fragmentierten Silt- und Sandsteinen des Unterdevons, die im Raum Eckfeld/Manderscheid üblicherweise mit einem Korngrößenspektrum zwischen Mittelsilt und Feinsand vertreten sind. Es ist also von einer generell hohen Verfügbarkeit feiner Quarzkorngrößen im Bereich des nebengesteinsreichen Tephrarings auszugehen. Nach FÜCHTBAUER (1988b) können Quarzkörner mit einem Durchmesser unterhalb von $80 \mu \mathrm{m}$ durch Luftströmungen in Suspension gehalten werden. Von wenigen Ausnahmen abgesehen entspricht dieses in der Tat dem maximalen Durchmesser von Quarzkörnern innerhalb der Mikrofazieszone 1a: Quarz-Fazies. Somit ist auch die Winderosion des Kraterrandes als bedeutende Lieferquelle für Feindetritus in Betracht zu ziehen (NELSON 1967, PIRRUNG 
1998). Die Auswehung großer Mengen unkonsolidierter Tephra aus den Randbereichen des Kraters wurde rezent an dem 1977 entstandenen östlichen Ukinrek Maar (Alaska) beobachtet (BÜCHEL \& LORENZ 1993). Gelegentlich sind $<1 \mathrm{~mm}$ mächtige Horizonte von oft matrixgestützt eingelagerten Grobsilt- und Feinsandquarzkörnern (LT 1a-4) in die strukturlosen Sedimentabschnitte eingeschaltet (Anhang 2-1, Fig. 04). Sie dokumentieren offensichtlich Intensitätsschwankungen im Eintrag von mineralischem Feindetritus (STURM 1979, PEDERSEN et al. 1998).

Homogene Pelite (LT 1a-1) gehen abschnittsweise in stärker laminierte Bereiche von mehreren Millimetern bis Zentimetern Mächtigkeit über (Anhang 2-1, Fig. 05 und Fig. 06). Hier alternieren zwischen $0,2 \mathrm{~mm}$ und 1,5mm mächtige quarzsilt- (LT 1a-2) und tonreiche Laminen (LT 1a-3). Beide Laminen-Typen führen feinsandgroße Quarzkörner. Im Mineralbestand gleichen die Hell/Dunkel-Wechselfolgen den unstrukturierten Peliten (Anhang 2-1, Fig. 01 und Fig. 02). Das eben parallele bis wellig parallele Schichtgefüge deutet jedoch auf einen Sedimentationsmechanismus hin, der zu einer Korngrößentrennung und zu der Anreicherung von Tonpartikeln in diskreten, meist unscharf begrenzten Horizonten führte. Ton/Silt-"couplets" in Seesedimenten sind nach GAYLORD et al. (2001) ein starker Hinweis auf einen temporär geschichteten Wasserkörper. Die Etablierung einer stabilen Thermokline wird in rezenten Seen niederer Breiten bereits durch einen geringen Temperaturunterschied von $1^{\circ}$ bis $2^{\circ}$ zwischen Epi- und Hypolimnion begünstigt (BURGIS \& MORRIS 1987, HUC et al. 1990). Aufgrund des Dichteunterschiedes zwischen beiden Wasserkörpern können sich Sedimentsuspensionen als "Interflows" entlang der Sprungschicht ausbreiten und feinkörniges Material über weite Seebereiche hinweg verteiIen (STURM \& MATTER 1978, StURM 1979, GallowAY \& HobDAY 1996). Während im Falle von Eckfeld sand- und siltgroße Quarzkörner kontinuierlich aus der Suspension ausfielen, wirkte die Epilimnion/Hypolimnion-Grenze offensichtlich als eine Art Sedimentfalle für Partikel geringerer Größe. Auch wenn tropische Seen die Tendenz zu einem geschichteten Wasserkörper aufweisen, so fördern doch bereits leichte Abkühlungen des Oberflächenwassers - beispielsweise durch die erniedrigte Insolation während Regenzeiten - das Einsetzen von Konvektionsströmen (GómeZ FERNÁNDEZ \& MELÉNDEZ 1991, TALBOT \& ALLEN 1996). In Eckfeld führte die episodische Durchmischung des Wasserkörpers offensichtlich zu einer Freigabe des an der Sprungschicht angereicherten Feinstmaterials und zur Ablagerung unreiner Tonlaminen jeweils im Hangenden einer siltreichen Lage (siehe auch STURM 1979). Die um den Faktor zehn schwankenden Mächtigkeiten sowohl der silt- als auch der tonreichen Laminen machen jedoch einen streng saisonal gesteuerten Wechsel von Stagnation und Zirkulation unwahrscheinlich. Es ist vielmehr davon auszugehen, daß die Turbulenzen zahlreicher, teils hochkonzentrierter Sedimentströme (siehe oben) sporadisch zu einer vertikalen Durchmischung des Wasserkörpers beitrugen. Insgesamt deuten alle sedimentologischen Befunde auf einen oligotrophen, warm polymiktischen See mit irregulären Zirkulationsmuster und einem anhaltenden Eintrag von mineralischem Feindetritus hin (BULLWINKEL \& RIEGEL 2001).

In Probe KS1 sind Pyritframboide mit rund 3 Vol.\% an der Sedimentzusammensetzung beteiligt und zeigen damit die drittgrößte Häufigkeit innerhalb der Bohrprofile von E1/96 und E2/96 (vgl. Anlage 2). Nach BERNER (1971) werden pyritreiche Süßwassersedimente generell in Milieus mit hohen Konzentrationen an gelöstem Sulfat gebildet. Unter Berücksichtigung auch der extremen Pyritgehalte unmittelbar im Hangenden der MFZ 1a: Quarz-Fazies (siehe unten) ist für den frühen Eckfeld-See von Sulfat-Konzentrationen auszugehen, die die durchschnittlichen Werte rezenter Seen (vgl. Kap. 6.5.1) um ein Vielfaches überschritten. Die hohen Ge- 
halte an Sulfat-Ionen lassen sich zum Teil auf verwitterte Sulfide aus den devonischen Untergrundgesteinen bzW. aus den angrenzenden Pyroklastiten zurückführen (MICKLICH \& WUTTKE 1988). In vielen vulkanogenen Seen sind die Austritte von Thermalwässern jedoch ebenfalls bestimmend für den Wasserchemismus (MORI et al. 1984, KELTS 1988, GAYLORD et al. 2001). Hydrothermale Quellen können auch im Eckfeld-See als eine mögliche Ursache für die erhöhte Zufuhr von Schwefel-Verbindungen angesehen werden. Daneben lieferten sie vermutlich einen Teil des für die Pyritgenese benötigten Eisens (BAHRIG 1988, LIEBIG 1998). Als weitere FeLieferanten kommen unverwitterte Primärminerale in Frage. Eisenhaltige Pyroxene und Amphibole wurden beispielsweise als häufige Einsprenglinge in den Tuffen von Eckfeld nachgewiesen (ZSÓTÉR 1997). Das Auftreten von Pyritframboiden in der MFZ 1a: Quarz-Fazies ist ein Beleg für anoxischen Bedingungen zumindest unterhalb der Sediment/Wassergrenze. Trotz der offensichtlichen Verfügbarkeit von Eisen und Sulfat war die Pyritbildung im Vergleich zur hangenden MFZ 2a: Quarz-Pyrit-Fazies jedoch eingeschränkt. Dieses ist zweifellos auf den allgemeinen Mangel an degradierbarer organischer Substanz im Sediment zurückzuführen. Das Fehlen organischer Partikel als Reduktionsmittel und Energiequelle für sulfatreduzierende Bakterien war in dieser frühen Seephase der limitierende Faktor für die Pyritgenese (vgl. Kap. 6.5.1).

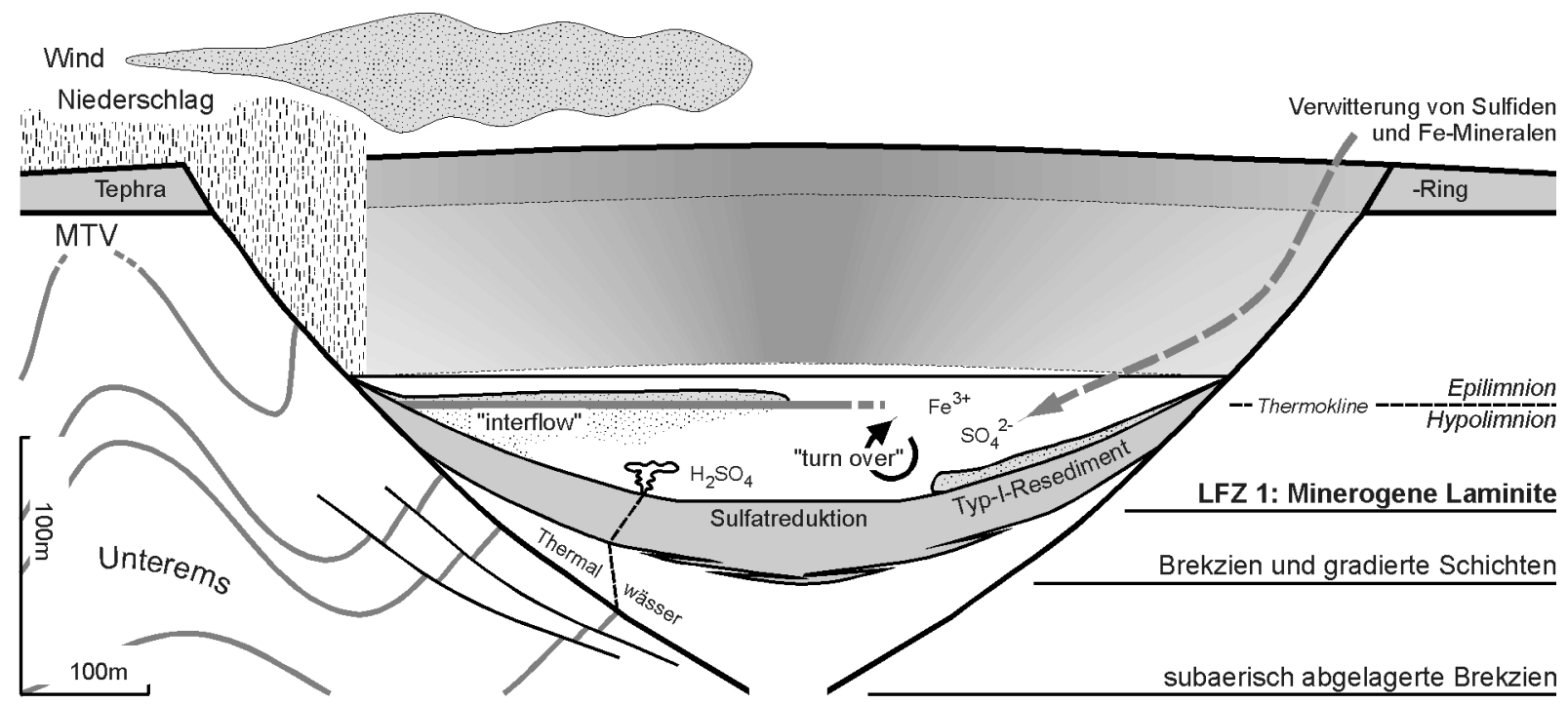

Abb. 24: Rekonstruktion der Sedimentationsmechanismen und der authigenen Mineralbildung im EckfeldSee zur Ablagerungszeit der LFZ 1: Minerogene Laminite.

\section{Lithofazieszone 2: Übergangsschichten}

Innerhalb der rund 50cm mächtigen MFZ 2a: Quarz-Pyrit-Fazies dominiert Pyrit mit über 85 Vol.\% (Probe KS2) bzw. knapp 48 Vol.\% (Probe KS3) die Sedimentzusammensetzung (Anhang 2-1, Fig. 07 und Fig. 08). Der außergewöhnliche Pyritreichtum korreliert mit dem stratigraphisch ersten Nachweis von Primärproduzenten und von organischem Detritus in nennenswerten Quantitäten (vgl. Anlage 2). Ein Teil der organischen Substanz wurde sicherlich bereits im Epilimnion unter weitgehend aeroben Bedingungen abgebaut (KATZ 1990). Das Einsetzen der organischen Sedimentation im Eckfelder Maar löste aber insbesondere eine rasche und intensive mikrobielle Reduktion des im Seewasser angestauten Sulfat-Reservoirs (siehe oben) aus. Bei einer hinreichend hohen Verfügbarkeit von Sulfat kann der mikrobielle Abbau von organi- 
scher Substanz unter anoxischen Bedingungen eine Größenordnung erreichen, die sonst nur aus oxischen Milieus bekannt ist (KELTS 1988). Im Falle von Eckfeld entstanden nahezu vollständig pyritisierte Sedimentabschnitte aus meist strukturlosen Einzelkristall-Akkumulationen (LT 2a-1, vgl. Kap. 4.2.1). In einigen Profilbereichen werden leistenförmige, mehrere 100um lange Framboid-Aggregate von geringmächtigen Turbiditen direkt und konkordant überlagert. Die Polyframboide wurden im Zuge der Sedimentkonsolidierung durch Devonklasten innerhalb der hangenden Turbidite verformt und zeichnen offensichtlich ehemalige Sedimentoberflächen nach, die durch synsedimentär gebildete Fe-Sulfide weitgehend verfestigt waren als die Trübeströme zur Ablagerung kamen. Es ist also von einer permanenten Redox-Linie auszugehen, die zu diesem Zeitpunkt an oder bereits oberhalb der Sediment/Wasser-Grenze lag (vgl. Kap 6.5.1). Neben Schwefelwasserstoff und Hydrogenkarbonat aus der bakteriellen Sulfatreduktion trug insbesondere die Anreicherung von reduziertem Eisen im anoxischen Tiefenwasser zu der Etablierung einer stabilen Dichteschichtung bei (KJENSMO 1968, MöRS 1995, LUTZ 1998, LUTZ et al. 2000). Nach GOTH (1990) war die bodennahe Akkumulation von Eisen(II)hydrogenkarbonat ebenfalls für eine permanente Meromixis im Messel-See ausschlaggebend.

In den pyritreichen Sedimenten der MFZ 2a ist also der Beginn einer meromiktischen Seephase dokumentiert. Verbunden mit dem kontinuierlich zunehmenden Eintrag von organischer Substanz führte die permanente Trennung eines stagnierenden, anoxischen Monimolimnions von einem zirkulierenden oxischen Mixolimnion zu der Ablagerung organisch-reicher Laminite im Hangenden der Lithofazieszone 2 (siehe unten).

Auffallend ist der rasche Anstieg von Primärproduzenten innerhalb der MFZ 2a. Während Botryococcus in der Liegenden Lithofazieszone 1 nicht nachgewiesen wurde, dominiert die Grünalge in diesem Profilabschnitt klar die organische Sedimentzusammensetzung (vgl. Anlage 2). Dieser Umstand spricht für eine grundlegende Veränderungen im Wasserchemismus der photischen Zone. Der schwefelreiche Eckfeld-See war in seiner Initialphase ein offensichtlich lebensfeindliches Habitat. Aufgrund der allgemein hohen Konzentrationen von Schwefel- und Salzsäure weisen viele vulkanogene Seen extrem niedrige $\mathrm{pH}$-Werte unterhalb von 4 auf (MORI et al. 1984, KELTS 1988, TALBOT \& ALLEN 1996). Während diese Bedingungen auf Phytoplankton offensichtlich letal wirkten, wurde der anaerobe Abbau von organischer Substanz durch Sulfatreduzierer rezent bereits unterhalb von pH 2 nachgewiesen (SATAKE 1977, TRUDINGER 1992). Erst durch den kontinuierlichen Entzug schwefliger Verbindungen aus der photischen Zone und deren Fixierung als Pyrit im Sediment bzw. als $\mathrm{H}_{2} \mathrm{~S}$ im anoxischen Monimolimnion verbesserten sich offenbar die Lebensbedingungen für Phytoplanktonvertreter. Botryococcus toleriert ein weites Spektrum ökologischer Parameter, insbesondere $\mathrm{pH}-$ Werte oberhalb von 4 (vgl. Kap. 6.4.1). Diese Eigenschaft ermöglichte es der Grünalge relativ früh, den Eckfeld-See zu besiedeln.

Der erste Nachweis von huminitischem Detritus innerhalb der MFZ 2a (vgl. Anlage 2) markiert die zunächst spärliche Besiedlung des Tephrawalls durch Landpflanzen. Zusammen mit den Huminiten steigen auch die Werte für Sporinit, der in der liegenden Lithofazieszone 1 nur mit seltenen Einzelexemplaren vertreten ist. Der überwiegende Polleneintrag entstammt somit nicht dem weiteren Umland, sondern ging von Mutterpflanzen mit Standorten im Bereich des Kraterrandes aus. 
Trotz der kontinuierlich steigenden Gehalte von huminitischem Pflanzendetritus im Sediment bleibt der siliziklastische Eintrag innerhalb der Lithofazieszone 2 signifikant. Im Bereich der MFZ $2 b$ wechsellagern humodetrinitreiche Tonlaminen ( $L T 2 b-1)$ mit $<1 \mathrm{~mm}$ mächtigen, teils extraklastenführenden Quarzkornlagen (LT 2b-3, vgl. Kap. 4.2.2). Die dunklen organisch-reichen Horizonte sind ihrerseits durch eine feine, makroskopisch kaum wahrnehmbare Internlamination gekennzeichnet (Anhang 2-2, Fig. 01 und Fig. 02). Sie geht auf lediglich wenige Korndurchmesser mächtige Lagen und Linsen von meist siltgroßen Quarzkörnern zurück (LT 2b-2). Die klastenführenden Quarzkornlagen (LT 2b-3) können trotz einer fehlenden bis lediglich schwach entwickelten Normalgradierung als distale Turbidite angesprochen werden (STURM \& MATTER 1978, BULLWINKEL \& RIEGEL 2001). Ein ähnlicher Ablagerungsmechanismus ist für die feinen Quarzsiltlagen und -linsen (LT 2b-2) innerhalb der organisch-reichen Horizonte wahrscheinlich. Nach COLLINSON \& THOMPSON (1989) gehen Siltlagen mit einer Mächtigkeit von nur wenigen Korndurchmessern oftmals aus gering konzentrierten Dichteströmen hervor, während assoziierte Tonlagen eine kontinuierliche Hintergrundsedimentation repräsentieren. Die Lithofazieszone 2 schließt zum Hangenden mit einem rund 2,6m mächtigen Typ-II-Resediment ab (vgl. Anlage 1). Das Konglomerat an der Basis dieses grobklastischen Profilbereichs ( $X_{1}$-Abschnitt, vgl. Kap. 5.1.2) beinhaltet außergewöhnlich große Extraklasten aus devonischen Siltsteinen und verfestigten Pyroklastiten mit minimalen Durchmessern von rund $60 \mathrm{~cm}$. Offenbar war der Maarsee in dieser frühen Phase weiterhin durch periodische Uferabbrüche von teils erheblichen Ausmaßen geprägt.

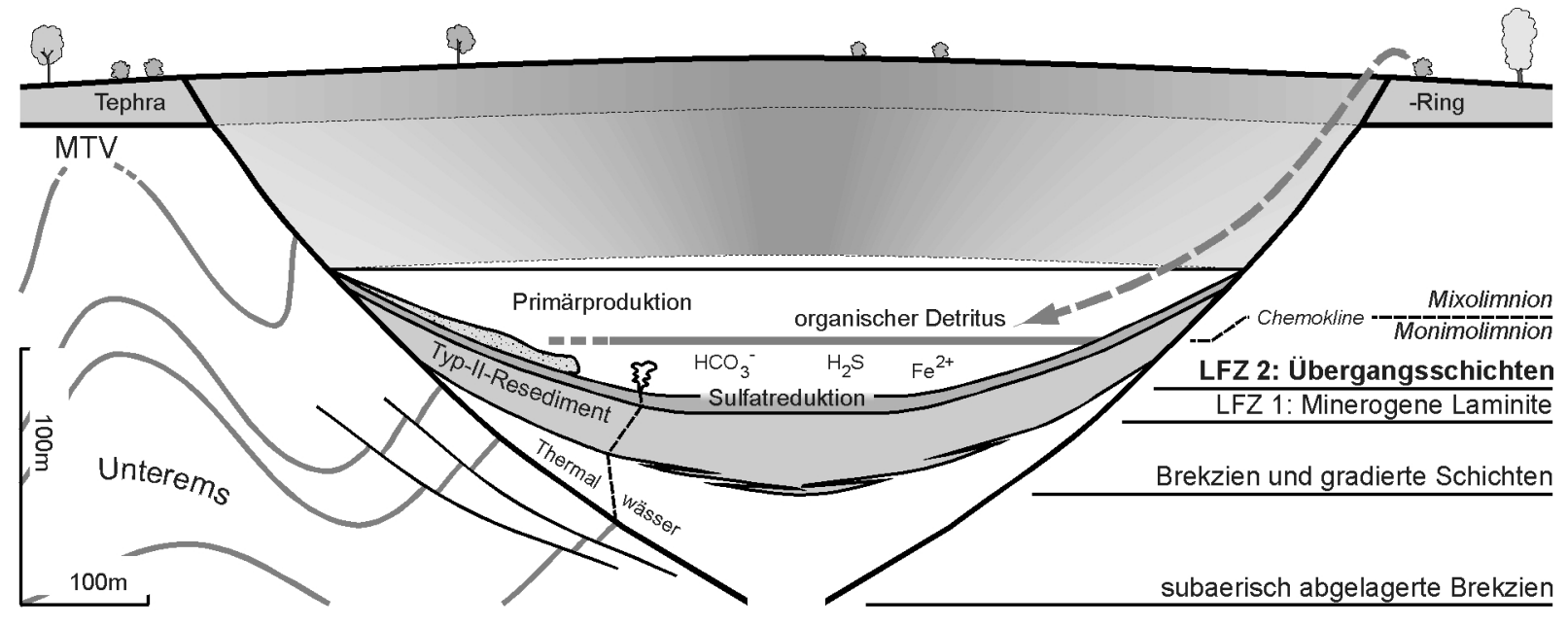

Abb. 25: Rekonstruktion der Sedimentationsmechanismen und der authigenen Mineralbildung im EckfeldSee zur Ablagerungszeit der LFZ 2: Übergangsschichten.

\section{Lithofazieszone 3: Biogene Laminite}

Die für die liegende Mikrofazieszone typischen Quarzkornlagen (LT 2b-2 bzw. LT 2b-3) verlieren innerhalb der Mikrofazieszone 3a: Huminit-Fazies zunehmend an Bedeutung. Mit der Abnahme siliziklastischer Einschaltungen geht ein deutlicher Anstieg der organischen Sedimentkomponente einher (vgl. Kap. 4.3.1). Organische Partikel sind in Probe KS9 mit über 50 Vol.\% am Sedimentaufbau beteiligt und weisen damit den höchsten Wert innerhalb der gesamten Profilabfolge von E1/96 und E2/96 auf (vgl. Anlage 2). In den untersten rund 15m der 
Biogenen Laminite wird das Mazeralspektrum deutlich von huminitischem Detritus dominiert. Obwohl Botryococcus als Primärproduzent in nahezu jeder Probe nachgewiesen wurde, fällt das Huminit:Liptinit-Verhältnis erst mit dem gehäuften Auftreten von Tetraedron in MFZ 3e: Tetraedron-Opal(CT)-Fazies auf einen Wert unterhalb von 1 (ab Probe KS37). Auffällig ist der generell geringe Durchmesser von Huminiten innerhalb der beckenzentralen Laminite. Unter den allochthonen organischen Partikeln nehmen siltkorngroße Humodetrinite, Detro-Gelinite und isolierte Corpohuminite in allen untersuchten Proben eine deutliche Vormachtstellung ein. Auch die Größe der pflanzlichen Gewebereste (Texto- und Eu-Ulminite) überschreitet in peliti-

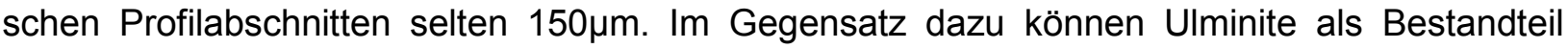
gröberklastischer Resedimente der Typen II und III (vgl. Kap. 5.1.2 und 5.1.3) Längen von mehreren Zentimetern erreichen. Hierin spiegelt sich offenbar eine hydrodynamische Sortierung von Organoklasten nach der Korngröße wider (HUC 1988). Größere Gewebereste kamen bereits im verhältnismäßig bewegten Litoral zur Ablagerung und wurden lediglich als Bestandteil von Sedimentströmen in tiefere Bereiche des Sees verlagert (WILDE \& FRANKENHÄUSER 1998). Organischer Feinstdetritus blieb dagegen länger in Suspension. Aufgrund der geringeren Dichte zwischen 1,3 und $1,6 \mathrm{~g} / \mathrm{cm}^{3}$ ist die Verweildauer von terrigenem organischen Material in der Wassersäule vergleichsweise lang (PRESTON 1974, FAHN 1982, LITTKE 1993). Turbulenzen in den oberen Wasserschichten behindern zusätzlich das direkte Absinken von organischem Detritus (HUC et al. 1990, OLSEN 1990). Im Falle von Eckfeld wurden kleinere PflanzenFragmente bis in zentrale Bereiche des Sees verfrachtet und sedimentierten unter Stillwasserbedingungen erst innerhalb des stagnierenden Monimolimnions.

Neben den erhöhten Anteilen von organischen Partikeln im Sediment ist die Lithofazieszone 3 auch durch das Auftreten von frühdiagenetisch gebildetem Siderit in Form von maximal $300 \mu \mathrm{m}$ mächtigen Horizonten oder isolierten Linsen gekennzeichnet (vgl. Kap. 4.3 und Kap. 6.5.2). Nach BERNER (1981a, b) bezeichnet das gemeinsame Auftreten von Siderit und Pyrit im Sediment anoxisch-nichtsulfidisch-methanische Milieubedingungen. Die extrem positiven $\delta^{13} \mathrm{C}-$ Werte von Fe-Karbonat deuten ebenfalls auf eine ausgeprägte mikrobielle Methanogenese in dieser Seephase hin (BAHRIG 1989). Vereinfacht läßt sich die Fermentation von organischer Substanz durch methanproduzierende Bakterien durch die Formel

$2 \mathrm{CH}_{2} \mathrm{O}[\mathrm{SOM}] \quad \rightarrow \mathrm{CH}_{4}+\mathrm{CO}_{2}$

beschreiben (BERNER 1981b, KATZ 1990). Während das leichtere ${ }^{12} \mathrm{C}$-Isotop vorwiegend als Methan gebunden wird, reichert sich ${ }^{13} \mathrm{C}$ in Form von $\mathrm{CO}_{2}$ im Porenwasser an, wo es für die Sideritbildung zur Verfügung steht (BAHRIG 1989). Die mikrobielle Methanproduktion findet unter stark reduzierenden Bedingungen (KATZ 1990), jedoch erst nach der weitgehenden Reduzierung von Sulfat und der Genese von Fe-Sulfiden statt (vgl. Kap. 6.5.1). Ein Grund dafür ist die geringere Effizienz methanogener Bakterien gegenüber Sulfatreduzierern (BERNER 1981b, HANSELMANN 1989). Im Falle von Eckfeld führte die Oxidation von $\mathrm{CH}_{4}$ an der Grenze zum oxischen Mixolimnion sehr wahrscheinlich zu einer Ausweitung des Sauerstoffdefizits innerhalb der Wassersäule (siehe KATZ 1990).

Die ersten diatomeenreichen Horizonte erscheinen ab Bohrmeter 31,8 (E1/96) bzw. 31,38 (E3/96). Innerhalb der MFZ 3b: Aulacoseira-Fazies alternieren maximal 1,5mm mächtige Dia- 
tomeenlagen ( $L T$ 3b-1) mit geringer mächtigen organisch-reichen Tonlaminen ( $L T$ 3b-2, vgl. Kap. 4.3.2). Die zentrische Diatomee Aulacoseira cf. granulata dominiert bei Weitem das Spektrum der nachgewiesenen Kieselalgen. Ihre ökologischen Ansprüche lassen auf einen eutrophen, Si-reichen See mit leicht alkalischem (etwa im Bereich von pH 8) Mixolimnion schließen (vgl. Kap. 6.3.1). Diese Einschätzung wird durch palynologische Untersuchungen von NICKEL (frdl. schriftl. Mitt. 2001) gestützt. Sie registrierte in diesem Profilabschnitt ein regelmäßiges Vorkommen von Ephedraceen-Pollen. Ephedraceen besiedeln heute alkalische, teilweise sogar versalzene Böden meist trockener und stark von der Sonne beschienener Standorte. Denkbar wären solche Extrembedingungen auf unbewachsenen Flächen am Kraterrand, die durch sedimentologisch nachgewiesene Rutschungen immer wieder freigelegt wurden (NICKEL, frdl. schriftl. Mitt. 2001).

Die Konzentrationen von Aulacoseira-Schalen in nahezu monospezifischen Lagen (Anhang 2-2, Fig. 07 und Fig. 08 bzw. Anhang 2-3, Fig. 01 bis Fig. 06) belegen episodische Massenentfaltungen der Kieselalge mit hohen Reproduktionsraten. Nach DECKER (1991) und BEHRENSMEYER et al. (1992) fördert die Zufuhr von Nährstoffen und gelöstem $\mathrm{SiO}_{2}$ die Entwicklung von Diatomeen-Blüten. In den pliozänen Seesedimenten des Yallalie-Impaktkraters (Südwest Australien) konnten DODSON \& RAMRATH (2001) allerdings keinen Zusammenhang zwischen der Häufigkeit von Diatomeen und dem Eintrag von Nährstoffen nachweisen. Sie führen die teilweise von Aulacoseira-Arten dominierten Planktonblüten vorwiegend auf Schwankungen der Salinität und der Wassertiefe zurück. Im periodischen Wechsel von Niederschlagsund Evaporationsphasen unter tropischen bis subtropischen Klimabedingungen dürften diese Faktoren auch eine maßgebliche Rolle im isolierten Eckfeld-See gespielt haben (BAHRIG 1989).

Auffällig ist, daß sich das Vorkommen von Botryococcus und Diatomeen-Massenansammlungen gegenseitig ausschließt (Anhang 2-3, Fig. 06). Botryococcus-Kolonien konnten lediglich in den organisch-reichen Horizonten zwischen zwei Diatomeenlagen nachgewiesen werden. Aufgrund der weiten Toleranz der Grünalge gegenüber ökologischen Parametern ist die Abwesenheit von Botryococcus in Diatomeenlagen kaum als eine direkte Reaktion auf saisonal schwankende Klimabedingungen zu werten, sondern erklärt sich vielmehr aus einer im Vergleich zu Diatomeen niedrigeren Wachstumsrate (vgl. Kap. 6.4.1). Im Wettbewerb um verfügbare Nährstoffe konnte sich Botryococcus offensichtlich nur in Zeiten entwickeln, in denen die Konkurrenz durch Diatomeen minimal war (siehe auch TYSON 1995, S. 312).

Im Gegensatz zu den hangenden, diatomeenführenden Mikrofazieszonen (MFZ 3d: Aulacoseira-Opal(CT)-Fazies und MFZ 3e: Tetraedron-Opal(CT)-Fazies) zeichnen sich die Diatomeen-Laminite der MFZ 3b durch eine diagenetisch unveränderte Erhaltung von Skelettopal (Opal-A) aus. In diesem Profilabschnitt konnten rasterelektronenmikroskopisch keinerlei Lösungserscheinungen an kieseligen Gehäusen beobachtet werden (Anhang 2-3, Fig. 02). Diese im Vergleich zu anderen limnischen Tertiärvorkommen Mitteleuropas bemerkenswert gute Überlieferung führt SCHILLER (1999) auf die Alternation von Diatomeenlagen mit organischreichen Peliten zurück, welche eine Barriere für zirkulierende Porenlösungen darstellen. Wechsellagerungen von opal- und tonreichen Laminen bilden jedoch ein generelles Sedimentationsmuster diatomeenführender Profilabschnitte und bauen auch die opaldiagenetisch überprägten Mikrofazieszonen 3d und 3e auf (vgl. Kap. 4.3.4 und 4.3.5). Die eingeschränkte Wasserwegsamkeit durch zwischengeschaltete Tonlagen kann also nicht als ein alleiniges Modell für die exzellente Erhaltung von Diatomeen-Frusteln in MFZ 3b dienen. Einer der wichtigsten Kontroll- 
faktoren für die Löslichkeit von $\mathrm{SiO}_{2}$ ist der pH-Wert (ILER 1979, BARKER et al. 1994). Ebenso wie in den Seesedimenten von Rott/Siebengebirge (MÖRS 1995) ist daher ein vorwiegend vom pH-Wert des See- und Porenwassers abhängiger Erhaltungsgrad kieseliger Mikrofossilien vorauszusetzen (vgl. Kap. 6.3.1). Aufgrund der zunehmenden Dissoziation von Kieselsäure steigt die Lösungsrate von $\mathrm{SiO}_{2}$ oberhalb von pH 9 in exponentiellem Maße an (FüCHTBAUER 1988a, BARKER et al. 1994, TUCKER 2001). Untersuchungen an rezenten Diatomeen-Schalen belegen darüber hinaus eine erhöhte Löslichkeit von Skelettopal bereits ab pH 7 (LEWIN 1961). Nach LOHMANN (1960) ist die Erhaltung kieseliger Frusteln über geologische Zeiträume hinweg möglich, sofern pH-Werte von 4 bis 5 im Sediment nicht überschritten werden. Für die Eckfelder Diatomeen-Laminite kann somit ein Porenwassermilieu etwa im neutralen bis moderat sauren $\mathrm{pH}$-Bereich angenommen werden. Die im Vergleich zum alkalischen Mixolimnion (siehe oben) niedrigeren $\mathrm{pH}-$ Werte beruhen vermutlich in erster Linie auf der anaeroben Zersetzung von organischer Substanz. Für die diatomeenreichen Profilabschnitte der Bohrung von 1980 weisen die extrem positiven $\delta^{13} \mathrm{C}$-Werte von Siderit $(>+10)$ auf eine starke Methanogenese im Sediment und/oder oberhalb der Sediment/Wasser-Grenze (BAHRIG 1989). Aus der mikrobiellen Fermentation kann eine Anreicherung von $\mathrm{CO}_{2}$ bzw. dessen schwacher Säure $\mathrm{H}_{2} \mathrm{CO}_{3}$ resultieren (HANSELMANN 1989, SCHNEIDER et al. 1990, DAMNATI et al. 1992), insbesondere, wenn ein Ca-Karbonat-Puffersystem, wie in Eckfeld, fehlt. Die Wirkung des anaeroben Abbaus von organischer Materie auf den $\mathrm{pH}-$ Wert des Sediments ist jedoch weitgehend abhängig vom Ausmaß der $\mathrm{CO}_{2}$-Produktion sowie der Bildung von organischen Säuren relativ zum Gehalt an stickstoffhaltigen Basen (BERNER 1971). Unterhalb der photischen Zone wird mikrobiell produziertes $\mathrm{CO}_{2}$ nicht durch die Photosynthese-Aktivität von Phytoplankton verbraucht. Als Folge davon sind tiefere Bereiche der Wassersäule sowohl in Seen als auch im marinen Milieu oftmals durch vergleichsweise niedrige pH-Werte gekennzeichnet (BERNER 1971). In dieser noch frühen Phase der Schwarzpelitbildung ist allerdings auch der anhaltende Einfluß hydrothermaler Lösungen auf die Wasserchemie im Eckfeld-See in Betracht zu ziehen (NICKEL, frdl. schriftl. Mitt. 2001).

Die Mikrofazieszone 3b: Aulacoseira-Fazies schließt zum Hangenden hin mit einem Typ-IIIResediment $a b$. Es beinhaltet in seinem mittleren Teil $\left(X_{2}\right.$-Abschnitt, vgl. Kap. 5.1.3) eine Rutschungsbrekzie aus zerscherten und intern gefalteten Pelit-Bruchstücken (Anhang 2-8, Fig. 02 und Fig. 03). Die eingelagerten Pelite entsprechen sedimentpetrographisch den ungestörten Diatomeen-Laminiten im Liegenden des Rutschkörpers, sind also offenbar ebenfalls unter anoxischen Bedingungen abgelagert worden. Dieser Umstand gibt einen deutlichen Hinweis auf die Höhenlage der Chemokline und auf die flächenhafte Verbreitung diatomeenreicher Pelite am Seeboden. Angesichts der bis steingroßen Devonklasten an der Basis des Resediments ( $\mathrm{X}_{1}$-Abschnitt) kann von einem sehr ufernahen Entstehungsort der Massenverlagerung ausgegangen werden. Die mitgeführten Laminit-Bruchstücke belegen, daß die Bildung organischreicher Pelite nicht nur auf die zentralen Seebereiche beschränkt war. Offensichtlich lagen weite Teile der Kraterhänge innerhalb des anoxischen Monimolimnions. Eine Höhenlage der Chemokline oberhalb von Regionen deren Hangneigung für die Entstehung von Massenverlagerungen ausreichte erklärt den allgemeinen Reichtum an umgelagerten Schwarzpeliten innerhalb der Lithofazieszone 3: Biogene Laminite. Ab dieser Phase der See-Entwicklung waren marginal abgelagerte, organisch-reiche Laminite generell in Rutschungsprozesse (TypIII-Resedimente) eingebunden. 
Mit 2,65m stellt die Rutschungsbrekzie am Top der MFZ 3b die mächtigste ResedimentSequenz innerhalb der untersuchten Profilabfolge dar (vgl. Anlage 1) und ist in ähnlicher Mächtigkeit ebenfalls in der rund 60m südwestlich gelegenen Bohrung von 1980 (Teufe: 44,5m bis 42,5m) entwickelt (NEGENDANK et al. 1982). Der Rutschkörper breitete sich offenbar über weite Teile des zentralen Seebodens aus. Es ist kaum anzunehmen, daß ein subaquatisches Rutschungsereignis diesen Ausmaßes ohne direkte Wirkungen auf die Seeökologie geblieben ist. Tatsächlich ist der Sedimentabschnitt unmittelbar im Hangenden des Resediments (MFZ 3c: Huminit-Opal-Fazies, Probe KS17) durch einen Totalausfall des Phytoplanktons gekennzeichnet (vgl. Anlage 2). Die von der Massenverlagerung induzierten Turbulenzen hatten sehr wahrscheinlich eine partielle oder vollständige Durchmischung des bis dahin geschichteten Wasserkörpers zur Folge (LUTZ 2000, LUTZ \& NEUFFER 2001). Experimentell konnte dieser Prozeß von LAMBERT \& LÜTHI (1977) nachgewiesen werden. Ein rezentes Beispiel für aufdringendes Bodenwasser nach einem Rutschungsereignis ist aus dem Züricher See bekannt. Nach einem Uferabbruch im Jahre 1875 bemerkte ein Augenzeuge die Trübung des Wassers im gegenüberliegenden Randbereich des Sees (HSÜ \& KELTS 1985). Durch die Auflösung der Dichteschichtung stieg anoxisches Tiefenwasser zusammen mit toxischen Verbindungen, wie $\mathrm{H}_{2} \mathrm{~S}$ und $\mathrm{CH}_{4}$, in die photische Zone auf. Nach LUTZ (1998) und GRUBER \& SCHÄFER (2000) kann die episodische Durchmischung des Eckfeld-Sees auch zu zeitweise erhöhten Gehalten an physiologisch wirksamen Elektrolyten (Eisenhydrogenkarbonat, Hydrogensulfid und Ammonium) im Mixolimnion geführt haben. Stenotop limnische Arten starben im Extremfall völlig ab, während sich euryhaline Arten uneingeschränkt vermehren konnten. Ein Indiz dafür ist das erneute Einsetzen von Botryococcus schon ab Probe KS18. Der Nachweis von Diatomeen fehlt dagegen über die gesamte Abfolge der MFZ 3c: Huminit-Opal-Fazies hinweg. Ob Letzteres tatsächlich temporär ungünstige Lebensbedingungen für Aulacoseira widerspiegelt, oder ob die Mischung mit alkalischen Oberflächenwasser (siehe oben) zu höheren $\mathrm{pH}-W$ erten im Tiefenwasser und somit zu einer verstärkten Lösung von Kieselschalen führte, kann nicht abschließend geklärt werden. Unter Berücksichtigung des Reichtums an Diatomeen-Resten und Schwamm-Spiculae in den angrenzenden Mikrofazieszonen ist das gleichzeitige Aussetzen sämtlicher kieseliger Mikrofossilien in diesem Profilabschnitt jedoch bemerkenswert. Das Fehlen auch von Gehäuseund Skleren-Abdrücken im Sediment deutet zusammen mit den Korrosionserscheinungen an der einzigen nachgewiesenen Chrysophyceen-Zyste auf eine weitgehende Lösung von biogenem Opal(A) bereits im Bereich der Sediment/Wasser-Grenze hin.

Einen Hinweis auf neutrale bis leicht alkalische Porenlösungen liefert das erstmalige Auftreten von Ca-Phosphat in diesem Profilbereich (vgl. Kap. 4.3.3 und Kap. 6.5.4). In der Bohrung von 1980 ist bereits der obere Abschnitt des liegenden Rutschkörpers durch Apatit stark verfestigt (NEGENDANK et al. 1982). Für die chemischen Bildungsbedingungen von Ca-Phosphat in den lakustrinen Tertiärvorkommen von Eckfeld, Messel und Rott/Siebengebirge gehen MICKLICH \& WUTTKE (1988), WUTTKE (1988) und MÖRS (1995) von sehr niedrigen oder fehlenden Sauerstoffgehalten und hohen Konzentrationen an Phosphat im Porenwasser sowie von einem $\mathrm{pH}-$ Wert zwischen 7 und 9 aus.

Die folgende MFZ 3d: Aulacoseira-Opal(CT)-Fazies ist durch das erneute Auftreten von Diatomeen-Resten gekennzeichnet (Anhang 2-5, Fig. 01 bis Fig. 03). In bezug auf das primäre Schichtgefüge gleichen die Alternationen aus diatomeenreichen Lagen ( $L T$ 3d-1) und organisch-reichen Tonlaminen (LT 3d-2) den Sedimenten der MFZ 3b: Aulacoseira-Fazies. Im 
Gegensatz zu diesen zeigen die $10 \mu \mathrm{m}$ bis $250 \mu \mathrm{m}$ mächtigen Diatomeenlagen jedoch eine fortgeschrittene Lösung von Skelettopal (vgl. Kap. 4.3.4). Die für die MFZ 3b spezifischen, exzellenten Erhaltungsbedingungen für Opal(A) waren in dieser Seephase nicht mehr gegeben. Eine frühdiagenetische Lösung von Diatomeen-Schalen fand sehr wahrscheinlich bereits wenige Zentimeter unterhalb der Sediment/Wasser-Grenze statt (vgl. Kap. 6.3.1).

Im Vergleich zu den stark schwankenden Werten in den liegenden Profilbereichen zeichnet sich die MFZ 3d durch ein relativ gleichbleibendes Huminit:Liptinit-Verhältnis zwischen 4,6 (Probe KS24) und 1,2 (Probe KS33) aus. Die sich bereits innerhalb der MFZ 3c abzeichnende Tendenz hin zu niedrigeren Werten in Richtung Hangendes setzt sich allerdings auch weiterhin fort und ist unmittelbar mit der abnehmenden Häufigkeit und Mächtigkeit im Profil dokumentierter Schüttungsereignisse korreliert (vgl. Anlage 1 und 2). Resedimente (Typ-III, vgl. Kap. 5.1.3) mit einer Mächtigkeit von mehr als $5 \mathrm{~cm}$ treten in den Mikrofazieszonen 3c, 3d und 3e nur noch selten auf und führen vergleichsweise wenig Devonklasten. Statt dessen bilden intraklastenreiche Tonlagen von weniger als $2 \mathrm{~mm}$ Mächtigkeit einen häufigen Laminen-Typ. Diese meist ungradierten Ereignislagen sind makroskopisch jedoch kaum von den ungestörten Laminiten im Liegenden und Hangenden zu unterscheiden. Insgesamt belegen die sedimentologischen und organisch petrologischen Befunde den sich kontinuierlich verringernden Eintrag von organischem Detritus und Fremdgesteinsfragmenten. Dieses kann auf eine fortschreitende Stabilisierung der Kraterwände durch eine geschlossene Pflanzendecke zurückgeführt werden (BULLWINKEL \& RIEGEL 2001). Zudem wirkten sich die häufigen und vielfach großräumigen Sedimentverlagerungen früherer Seephasen ausgleichend auf die Beckenmorphologie aus und hatten eine kontinuierliche Abnahme der Reliefenergie im Ablagerungsraum zur Folge. Innerhalb der MFZ 3d äußert sich der progressive Reliefausgleich ebenfalls in der Zusammensetzung des überlieferten Pollenspektrums. Der regelmäßige Nachweis von Chloranthaceen-, Restionaceen- und Cyperaceen-Pollen spricht für eine Besiedlung der Seeränder durch eine teils krautige Ufervegetation (NICKEL, frdl. schriftl. Mitt. 2001).

Im Profilbereich der MFZ 3e: Tetraedron-Opal(CT)-Fazies sind die Gehäusereste von Aulacoseira cf. granulata nur noch vereinzelt und lediglich an der Basis der Abfolge nachzuweisen. Daß Diatomeen dennoch eine bedeutende Phytoplankton-Gruppe im Eckfeld-See darstellten ist durch häufige Schalenabdrücke im feinkörnigen Sediment belegt (Anhang 2-6, Fig. 04 und Fig. 05). Es ist also von einem weiterhin eutrophen, leicht alkalischen Mixolimnion auszugehen. Das teils individuenreiche Auftreten der Grünalge Tetraedron cf. minimum bestätigt diese Auffassung, da es sich bei den Vertretern der Gattung Tetraedron generell um eutrophe Arten zu handeln scheint (vgl. Kap. 6.4.1). Die drei für die MFZ 3e charakteristischen Phytoplankton-Formen Botryococcus cf. braunii, Tetraedron cf. minimum und Aulacoseira cf. granulata treten nebeneinander ebenfalls im leicht alkalischen Epilimnion des biochemisch geschichteten Toba-Sees (Sumatra) auf (RUTTNER 1952).

Nach palynologischen Analysen von NICKEL $(1994,1996)$ wurde der See in dieser Phase von einem artenreichen, durch Juglandaceen dominierten, thermophilen Wald umstanden. Die übergeordnete Rolle von Walnußgewächsen (Juglandaceae) äußert sich ebenfalls in der Bandbreite der in Eckfeld geborgenen Blattfossilien (FRANKENHÄUSER \& WILDE 1993, WILDE \& FRANKENHÄUSER 1998). Sehr wahrscheinlich war der See zu dieser Zeit, zumindest periodisch, an ein Gewässernetz angebunden. Dafür sprechen die Funde von Flußmuscheln (Unionidae) und Schnecken (Viviparidae) im Eckfelder Grabungsprofil sowie der Nachweis eines Fliegen- 
larven-Köchers (Trichoptera) (LUTZ 1988, GROH \& JUNGBLUTH 1994, NEUBERT 1995) ebenso, wie die erhöhten Frequenzen von vermutlich allochthonen Elementen (Pinaceen, Fagaceen) im Pollenspektrum (NICKEL, frdl. schriftl. Mitt. 2001). Ein erhöhter fluviatiler Eintrag von Nährstoffen könnte auch zu der teils massenhaften Entfaltung von Tetraedron in diesem Sedimentabschnitt beigetragen haben.

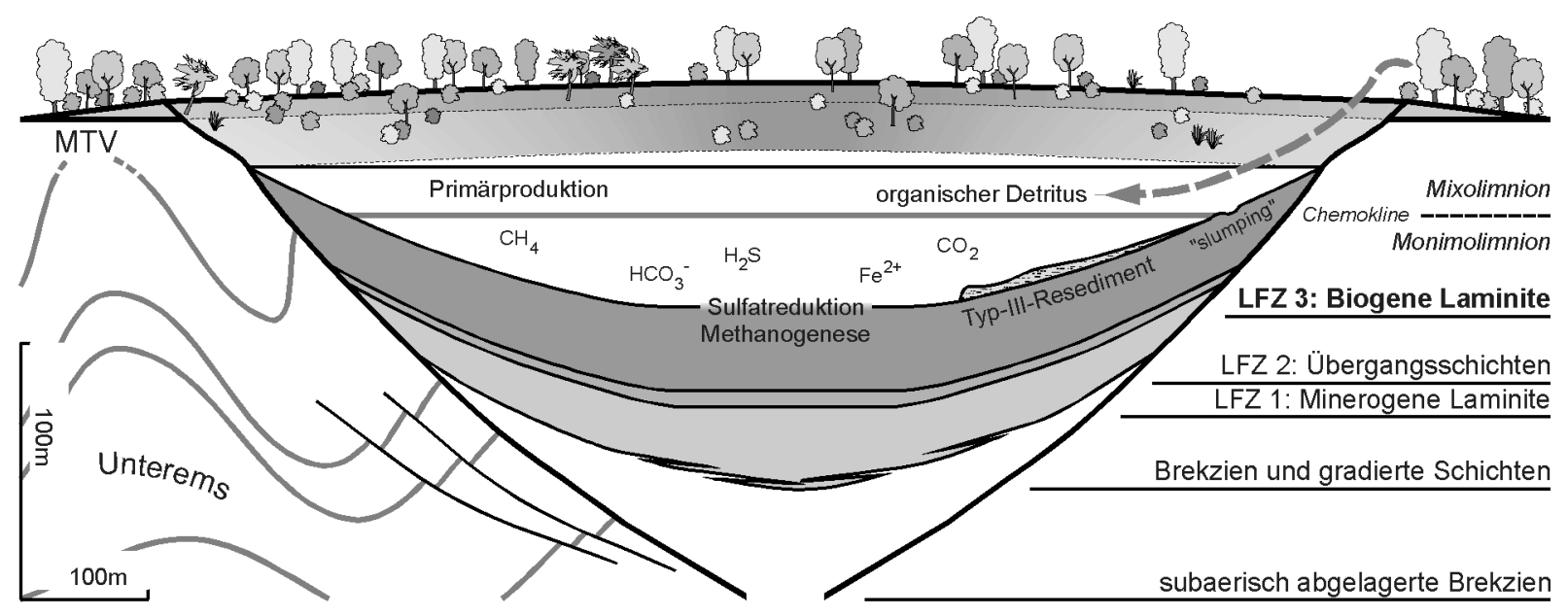

Abb. 26: Rekonstruktion der Sedimentationsmechanismen und der authigenen Mineralbildung im EckfeldSee zur Ablagerungszeit der LFZ 3: Biogene Laminite.

\subsection{Jahresrhythmik?}

Die Mikrofaziesanalyse an den zentralen Seesedimenten des Eckfelder Maares hat gezeigt, daß die Identifizierung eines möglicherweise klimagesteuerten Sedimentationsmusters nur an den rund 1,4m mächtigen Diatomeen-Laminiten der MFZ 3b: Aulacoseira-Fazies gelingen kann. In den liegenden Lithofazieszonen 1 und 2 sind die Seeablagerungen durch einen signifikanten siliziklastischen Eintrag und häufige Resedimentationsereignisse gekennzeichnet. Das Sedimentationsgeschehen wurde in dieser frühen Seephase maßgeblich durch eine noch steile Beckenmorphologie und die Nähe eines un- oder lediglich spärlich bewachsenen Tephrawalls als Sedimentquelle gesteuert. Es ist somit von einer starken Überprägung möglicher Klimasignale durch chaotisch periodische Sedimenteinschüttungen auszugehen.

Im Hangenden der MFZ 3b führte die Migration gelöster Kieselsäure aus Skelettopal zu einer Auflösung primärer Schichtgrenzen. Die frühdiagenetische $\mathrm{SiO}_{2}$-Umlagerung im Bereich der Mikrofazieszonen 3d: Aulacoseira-Opal(CT)-Fazies und 3e: Tetraedron-Opal(CT)-Fazies hatte eine postsedimentäre Mächtigkeitsabnahme von Tonlagen bei einer gleichzeitigen Mächtigkeitszunahme von opalreichen Horizonten zur Folge (vgl. Kap. 6.5.3).

Im wesentlichen sind drei Laminen-Typen am Aufbau der Diatomeen-Laminite in MFZ 3b beteiligt (vgl. Kap. 4.3.2). Zweifellos gehen die weitgehend monospezifischen AulacoseiraLagen (LT 3b-1) auf episodische Massenentfaltungen von Diatomeen zurück, wie sie beispielsweise aus dem Malawi-See (Ost-Afrika) beschrieben werden (CROSSLEY \& OWEN 1988). Die Produktivitätsphasen bildeten sich als Ereignislagen innerhalb der organisch-reichen Hintergrundsedimentation (LT 3b-2) ab. Unter 500 $\mu$ m mächtige Diatomeenlagen sind weitgehend frei von organischer Substanz und mineralischen Fremdpartikeln. Sie repräsentieren vergleichsweise kurze Algenblüten. Mächtigere Lagen beinhalten dagegen oftmals dünne Tonlinsen und 
isoliert eingelagerte Huminite. Dieses spricht für einen fortdauernden Eintrag von Ton und organischem Detritus auch während längerer Phasen der Diatomeen-Massenproduktion.

Die Alternationen aus diatomeen- und organisch-reichen Tonlagen werden in unregelmäßigen Abständen von $<2 \mathrm{~mm}$ mächtigen, intraklastenführenden Horizonten unterbrochen (LT 3b-3). Sie bestehen zu einem Großteil aus schichtparallel eingeregelten Laminit-Fragmenten und sind in der Regel nur im Dünnschliff von den klastenfreien Tonlagen zu unterscheiden. Gemäß ihrer Deutung als distale Endglieder von Typ-III-Resedimenten (vgl. Kap. 5.1.3) sind die klastenführenden Horizonte ebenfalls als Ereignislagen anzusprechen. Bei den Laminiten der MFZ $3 b$ handelt es sich also nicht nur um simple A:B sets. Die episodischen Ablagerungen intraklastenreicher Sedimentströme (LT 3b-3) führte vielmehr zu einem abrupten Anstieg der Sedimentationsrate. Dieses ist als mögliche Fehlerquelle für Spektralanalysen zu berücksichtigen.
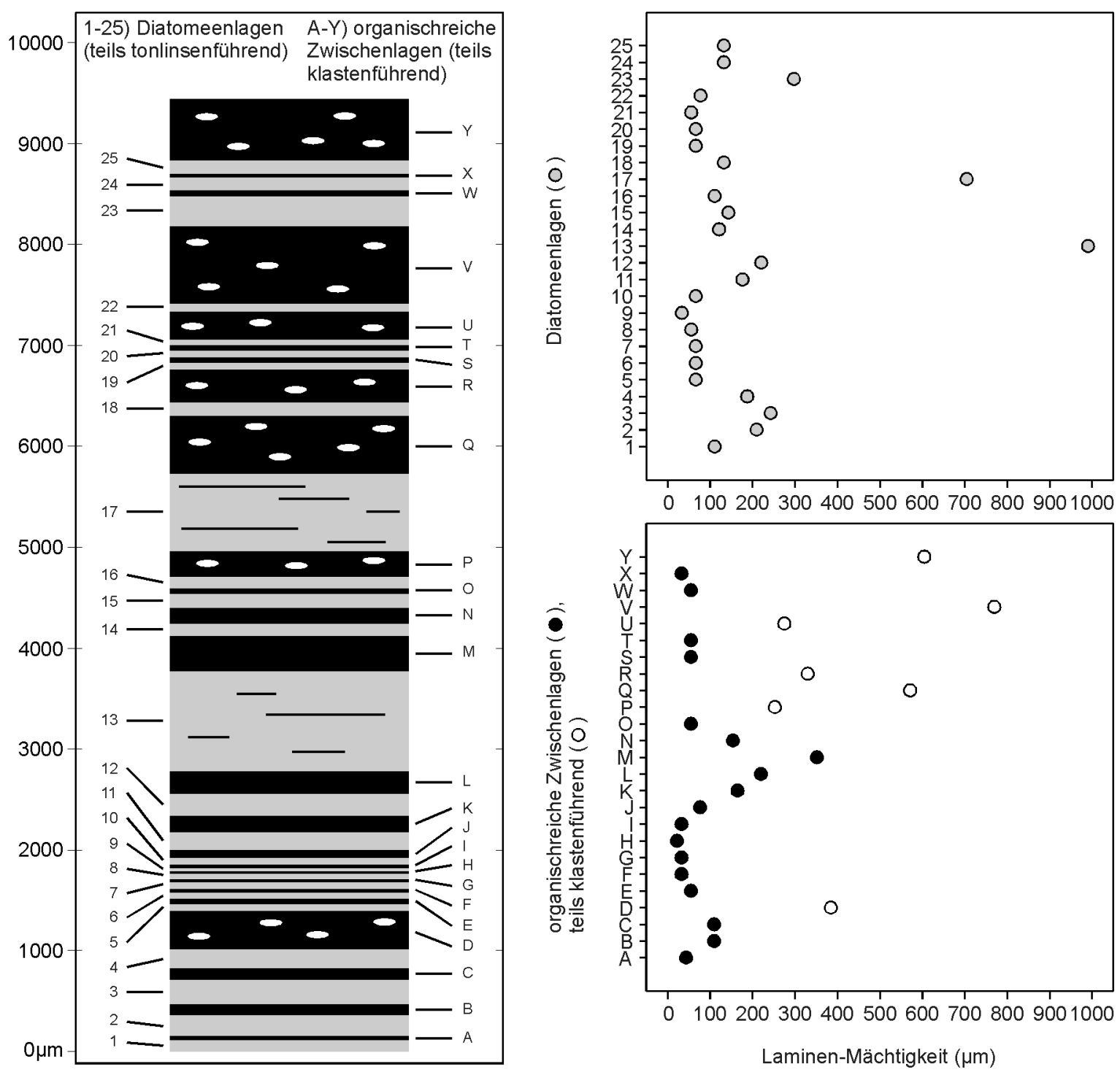

Abb. 27: Mächtigkeiten von Diatomeenlagen, organisch-reichen Tonlagen und klastenführenden Tonlagen innerhalb der Mikrofazieszone 3b: Aulacoseira-Fazies.

Innerhalb der MFZ 3b stehen die Mächtigkeiten der Diatomeenlagen (LT 3b-1) und der jeweils assoziierten Tonzwischenlagen (LT 3b-2) in einem engen Verhältnis zueinander. An 
einem rund $1 \mathrm{~cm}$ umfassenden Profilausschnitt (Probe BL/A-F1.2) wurden die absoluten Schichtmächtigkeiten von insgesamt 50 Laminen bestimmt (Abb. 27). Dabei zeigte sich, daß dünne Diatomeenlagen mit einer Mächtigkeit von $<100 \mu \mathrm{m}$ durch ähnlich geringmächtige Tonzwischenlagen unterbrochen werden. Es gilt: Mächtigkeit Diatomeenlage $=$ Mächtigkeit Tonlage $\mathrm{x} \mathrm{k} \mathrm{k}$ $=0,5$ bis 1]. Mit ansteigender Mächtigkeit einer diatomeenreichen Lage nimmt in aller Regel auch die Stärke der angrenzenden Tonschichten zu. Die Mächtigkeitsrelationen sind bei Diatomeen- und Tonlagen mit einer Schichtdicke $>100 \mu \mathrm{m}$ jedoch weniger streng ausgebildet und gelten in keinem Fall für die klastenführenden Tonhorizonte (LT 3b-3).

Die Mächtigkeitsschwankungen von Aulacoseira-Ereignislagen (LT 3b-1) und assoziierten Tonhorizonten (LT 3b-2) sind deutlich positiv korreliert (Abb. 27). Dieses ist in Anbetracht ihrer verschiedenartigen Genese bemerkenswert und kann plausibel nur über den Faktor Zeit erklärt werden. Unter der Voraussetzung, daß die klastenfreien Tonlaminen eine weitgehend kontinuierliche Hintergrundsedimentation repräsentieren und ihre jeweilige Mächtigkeit mit dem zeitlichen Abstand zwischen zwei Diatomeen-Blüten in bezug steht, zeigt die untersuchte Sedimentsequenz das Auftreten von Aulacoseira-Produktivitätsphasen in stark variierenden Frequenzen. Abschnittsweise spiegelt das Sedimentationsmuster schnell aufeinander folgende, vermutlich mehrmals im Jahr auftretende Diatomeen-Blüten von kurzer Dauer wider (beispielsweise Lamine 5 bis 10 in Abb. 27). Auf der anderen Seite belegen die über 500 $\mu$ m mächtigen, tonlinsenführenden Diatomeenlagen (Lamine 13 und 17 in Abb. 27) seltene aber weitaus länger andauernde Phasen einer optimalen Algenentwicklung.

Anhand der Mächtigkeitsverteilungen von Diatomeenlagen und assoziierten Tonhorizonten ist eine strenge Jahresrhythmik nicht unmittelbar nachzuvollziehen. Trotz einer von MAI (1995) für das Mitteleozän Europas postulierten Wintertrockenheit mit kurzen Dürreperioden scheint das Sedimentationsmuster vielmehr eine supersaisonale Zyklizität abzubilden (Abb. 27), die beispielsweise einen Wechsel von regenreichen und regenarmen Jahren andeutet. In subrezenten Sedimenten von Seen niederer Breiten ist die Signifikanz saisonaler Zyklen oftmals fraglich (VINCENT et al. 1986, HALFMAN \& JoHnSON 1988, POLLINGHER 1990, TILZER 1990, GIRESSE et al. 1991, DAMNATI et al. 1992, TALBOt \& AlLEN 1996). Auch für die mitteleozänen Seeablagerungen von Eckfeld läßt die vertikale Verteilung weder der Primärproduzenten noch der Sporomorphen Rückschlüsse auf eine Jahresrhythmik zu (NICKEL 1996, CLAUSING 2001). Botryococcus-Kolonien sind über den gesamten Abschnitt der untersuchten Profundalsedimente hinweg vorwiegend isoliert und statistisch im Sediment verteilt. Zwar kam es gelegentlichen zu Phasen der erhöhten Produktivität, eine Saisonalität ist anhand der sporadisch auftretenden Botryococcus-reichen Lagen jedoch nicht abzuleiten. Die Variationen in den Koloniedurchmessern von Botryococcus machen vielmehr Wachstumsperioden wahrscheinlich, deren jeweilige Dauer nicht signifikant von jahreszeitlichen Klimaschwankungen beeinflußt war (vgl. Kap. 6.4.1).

Demgegenüber halten MINGRAM $(1994,1997,1998)$ sowie Vos \& MINGRAM (2002) eine Warven-Bildung im Eckfeld-See für wahrscheinlich. Dieses vorausgesetzt ist mit einer Sedimentationsrate von $0,5 \mathrm{~mm} / \mathrm{a}$ für die Diatomeen-Laminite bzw. von 0,4mm/a für die diatomeenfreien Sedimentabschnitte zu rechnen (MINGRAM 1994, 1998). Geht man von einer Mächtigkeit der Laminite von ehemals $100 \mathrm{~m}$ aus (vgl. Kap. 2.3), würde sich für den Eckfeld-See eine theoretische Sedimentationsdauer von etwa 250000 a ergeben (PIRRUNG 1998, PIRRUNG et al. 2001). 


\subsection{Die Profundalsedimente von Eckfeld im Vergleich mit den Seeablagerungen von Messel (Mitteleozän) und Enspel/Westerwald (Oberoligozän)}

\section{Messel}

Die neue Forschungsbohrung "Messel 2001" durchteufte eine insgesamt 433m mächtige Abfolge aus Schwarzpeliten und Vulkaniklastika, die auf einen Maarsee als Ablagerungsraum schließen läßt (HARMS 2002). Zu dem gleichen Ergebnis kommt auch LIEBIG (2001) aufgrund einer Neubeschreibung der Bohrungen KB 1,2,4,5 und 7 von 1980. Unterhalb von 228m wird das Bohrprofil "Messel 2001" von geschichteten Brekzien, Lapillituffen und Tuffiten dominiert. Darüber folgen oft gradierte Lagen aus Feinkies, Sand und Ton. Sie markieren den Beginn der lakustrinen Sedimentation im Messel-See. Organisch-reiche Pelite setzen ab Bohrmeter 139 ein. Die laminierten Sequenzen werden jedoch immer wieder durch gradierte Feinschichten und Groblaminen unterbrochen (FELDER \& HARMS 2001).

In der generellen Abnahme grobklastischer Profilbereiche zugunsten laminierter Schwarzpelite in Richtung Hangendes entsprechen die Messeler Seeablagerungen den Profundalsedimenten von Eckfeld und deuten damit auf eine in Grundzügen ähnlich verlaufende Sedimentationsgeschichte beider Seen hin. PIRRUNG et al. (2001) kalkulieren einen Durchmesser des ehemaligen Messel-Sees von $900 \mathrm{~m}$ bis $1200 \mathrm{~m}$ und eine initiale Tiefe von rund $230 \mathrm{~m}$. Somit sind die Seen von Messel und Eckfeld auch in ihren Dimensionen vergleichbar.

Nach palynologischen Untersuchungen von NICKEL (1996) ist in beiden Mitteleozän-Vorkommen eine deutliche Vorherrschaft von Juglandaceen-Pollen zu verzeichnen. Bei einem summarischen Vergleich der Mikrofloren von Messel und Eckfeld ergibt sich trotz des unterschiedlichen Alters eine höhere Übereinstimmung als mit gleichalten, faziell aber stark abweichenden Strata, wie beispielsweise dem Geiseltal, Bouxwiller (Elsaß) oder dem Pariser Becken. Das im Vergleich zu Messel seltenere Auftreten von Restionaceen-Pollen in Eckfeld ist vermutlich eine Folge schmalerer Uferzonen (NICKEL 1996).

Die Volumengehalte von partikulärer organischer Substanz reichen in Messel von $30 \%$ bis 60\%. Davon machen meist in Lagen angereicherte Huminit-Mazerale im Mittel 25 Vol.\% aus (JANKOWSKI \& LITTKE 1986). Dieses entspricht in etwa dem Gehalt an Huminiten in der obersten Mikrofazieszone (MFZ 3e: Tetraedron-Opal(CT)-Fazies) von Eckfeld. In den liegenden Profilabschnitten sind Huminite sehr viel häufiger vertreten als in Messel. Sporinit, Cutinit und Resinit ist in Messel, ähnlich wie in Eckfeld, mit vergleichsweise niedrigen Werten von maximal 4 Vol.\% des organischen Materials repräsentiert. Bezogen auf den Gesamtgehalt an Mazeralen werden die Seeablagerungen von Messel mit 60 Vol.\% bis 90 Vol.\% deutlich von alginitischem Liptinit dominiert (JANKOWSKI \& LITTKE 1986). Im Gegensatz zu Eckfeld war die Grünalge Botryococcus jedoch nur untergeordnet an der Primärproduktion beteiligt (GOTH 1990; CLAUSING 2000, 2001). Als entscheidender Primärproduzent wurde die coccale Chlorophycee Tetraedron minimum identifiziert, die oftmals in nahezu reinen Algenlagen angereichert ist (GOTH 1990).

Trotz des Fehlens reiner Tetraedron-Lagen in Eckfeld und der stärkeren Präsenz von Botryococcus zeigen die Seeablagerungen von Messel in ihrem Gehalt an organischer Substanz starke Ähnlichkeit zu der MFZ 3e: Tetraedron-Opal(CT)-Fazies. Die relativ zu Messel weitaus höheren Gehalte an Huminiten in tieferen Bereichen der Eckfelder Profundalfazies sind auf einen stärkeren Eintrag von pflanzlichem Detritus zurückzuführen. Angesichts des weitgehend identischen Durchmesser:Tiefen-Verhältnisses von etwa 5:1 (PIRRUNG 1998, PIRRUNG 
et al. 2001) ist für beide Ablagerungsräume ein ähnlich starkes Relief anzunehmen. Die vergleichsweise hohen Gehalte an detritischen Huminiten in Eckfeld deuten jedoch darauf hin, daß die Kraterrandbereiche in der Frühphase der See-Entwicklung weniger stark verfestigt waren als in Messel.

\section{Enspel/Westerwald}

Die Fossilfundstätte Enspel liegt inmitten einer komplexen vulkanotektonischen Struktur (PIRRUNG 1998). Aufgrund des Verhältnisses von phreatomagmatischen Lapilli zu Ignimbriten oder Lava-Komponenten und der länglichen Form des Oligozän-Vorkommens nehmen PIRRUNG et al. (2001) eine kleine Caldera als Ablagerungsraum für die organisch-reichen Seesedimente an. Für den Enspel-See ist ein Durchmesser von 1,3km bis $1,7 \mathrm{~km}$ und eine initiale Tiefe von rund 240m wahrscheinlich (PIRRUNG et al. 2001).

In dem Bohrprofil "Enspel 1996" sind meist laminierte Schwarzpelite in einem Teufenbereich zwischen 2,6m und 142,0m erschlossen. Sie nehmen jedoch nur rund $20 \%$ der Abfolge ein. Die übrigen Ablagerungen werden durch Tuffe und Resedimente gebildet (FELDER et al. 1998, CLAUSING 2001). Der basaltische Lava-Strom eines nahegelegenen Eruptionszentrums beendete die aquatische Sedimentation und versiegelte die liegenden Seeablagerungen (CLAUSING 1998, PIRRUNG et al. 2001).

Im Vergleich zu Eckfeld ist das Spektrum nachgewiesener Primärproduzenten in Enspel sehr viel größer. Abgesehen von Tetraedron sind vor allem unstrukturierte Lamalginite am Laminationsaufbau beteiligt (CLAUSING 1998, MüNTER 1999). Letztere lassen sich nach CLAUSING (2001) auf Cyanobakterien zurückführen. Neben Botryococcus-Kolonien werden Telalginite vor allem durch meist lagenweise konzentrierte Dinoflagellaten-Zysten vertreten (CLAUSING 1998, 2000). In einigen Profilabschnitten alternieren diatomeenreiche Lagen mit Horizonten, die Chrysophyceen-Zysten führen (CLAUSING 2001). Im Gegensatz zu der von Aulacoseira cf. granulata dominierten Diatomeen-Flora von Eckfeld sind Kieselalgen in Enspel mit acht Taxa nachgewiesen (SCHILLER 2000, CLAUSING 2000).

Nach CLAUSING $(1998,2001)$ repräsentieren Laminitsequenzen mit dominierend silikatischen Produzenten (Diatomeen und Chrysophyceen) ein möglicherweise jahreszeitlich induziertes Sedimentationsmuster. Dieses wurde jedoch immer wieder durch unregelmäßige Phytoplanktonblüten überlagert. In der Art und der Verteilung von organischer Substanz weisen die Seesedimente von Eckfeld und Enspel nur wenig Gemeinsamkeiten auf. Ein Grund für die gegenüber Eckfeld erhöhte Primärproduzenten-Vielfalt in Enspel ist sicherlich in der zeitweise verstärkten Zufuhr von Nährstoffen durch episodische Tuffeinträge zu suchen.

\section{Zusammenfassung der Ergebnisse}

Die vorliegenden Untersuchungen sind Teil des interdisziplinären Gemeinschaftsprojektes "Bohrung Eckfelder Maar 1996" unter der Koordination des Naturhistorischen Museums Mainz/Landessammlung für Naturkunde Rheinland-Pfalz. Projektziel ist die detaillierte Rekonstruktion des Paläoökosystems "Eckfelder Maarsee".

Auf der Grundlage sedimentologischer, mikrofazieller und organisch-petrologischer Analysen an den beckenzentralen Bohrungen Eckfeld 1/96, 2/96 und 3/96 werden 15 Laminen-Typen 
(LT) beschrieben, die sich untereinander durch ihren Stoffbestand, ihre diagenetische Überprägung sowie durch den Charakter der daraus resultierenden Mikroschichtungsgefüge unterscheiden. Durch die Abgrenzung diskreter Laminen-Typen können grundlegende Bildungs- und Ablagerungsmechanismen von organischer und mineralischer Substanz aufgezeigt werden (Tab. 29).

Nach ihren internen Gefügemerkmale lassen sich die bis 2,65m mächtigen Resedimente der zentralen Seefazies in insgesamt fünf Textur-Abschnitte unterteilen, die offensichtlich Variationen in den Transport- und Ablagerungsmechanismen der Sedimentströme widerspiegeln. Das Auftreten bzw. Fehlen definierter Abschnitte läßt Rückschlüsse auf das transportenergetisch wirksame Relief des Ablagerungsraumes zu und gibt Hinweise auf den Ursprungsort der Massenverlagerungen (Tab. 30).

Anhand einer hochauflösenden Gliederung der Bohrprofile in drei Lithofazieszonen (LFZ) mit insgesamt acht Mikrofazieszonen (MFZ) wird die Sedimentationsgeschichte des mitteleozänen Eckfeld-Sees nachvollzogen.

\section{Lithofazieszone 1: Minerogene Laminite}

Teufe:

E1/96: $41,30 \mathrm{~m}$ bis $37,67 \mathrm{~m}$

E3/96: $39,20 \mathrm{~m}$ (Endteufe) bis $36,79 \mathrm{~m}$

Die MFZ 1a: Quarz-Fazies bildet die einzige Mikrofazieszone der LFZ 1: Minerogene Laminite. Mit 70\% der Gesamtmächtigkeit machen grobklastische, nebengesteinsreiche Resedimente (Typ-I-Resedimente, vgl. Tab. 30) den Hauptanteil der mineralischen Sedimentabfolge aus. Sie lassen sich aus "hyperconcentrated density flows" bzw. "concentrated density flows" ableiten, die distal in geringer konzentrierte "turbidity currents" übergingen. Die Häufigkeit der dokumentierten Sedimentverlagerungen belegt eine hohe Reliefenergie entlang der steilen, instabilen Kraterinnenwände. Ein Hauptmerkmal der MFZ 1a ist das weitgehende Fehlen von organischen Partikeln im Sediment. Dieser Umstand läßt auf einen noch unbewachsenen Tephrawall schließen, der den See in seiner Frühphase umgab und inn wirksam von einem Detrituseintrag aus dem Umland isolierte.

Den siliziklastischen Ereignislagen zwischengelagert sind feinsandige, teils laminierte, teils strukturlose Pelite. Sie zeigen keinerlei texturelle oder petrographische Merkmale eines lateralen Sedimenttransports und repräsentieren Ablagerungen aus einer feinkörnigen Sedimentsuspension. Die homogenen, quarzsandreichen Pelite kennzeichnen einen anhaltenden Eintrag von Feindetritus durch die kontinuierliche Erosion des Tephrawalls. Unscharfe Alternationen aus jeweils $0,2 \mathrm{~mm}$ bis $1,5 \mathrm{~mm}$ mächtigen tonarmen und tonreichen Siltlagen lassen zusätzlich auf einen Sedimentationsmechanismus schließen, der zu einer Anreicherung von Tonpartikeln in diskreten Horizonten führte. Die Ton/Silt-"couplets" sprechen für einen temporär geschichteten Wasserkörper. Während sand- und siltgroße Quarzkörner kontinuierlich aus der feinkörnigen Suspension ausfielen, wirkte die Epilimnion/Hypolimnion-Grenze als eine Sedimentfalle für Partikel geringerer Dichte. Ihre Akkumulation innerhalb der Wassersäule hatte die Ablagerung unreiner Tonlaminen während episodischer Durchmischungsphasen zur Folge. Die stark schwankenden Mächtigkeiten sowohl der silt- als auch der tonreichen Laminen machen einen 


\begin{tabular}{|c|c|c|c|c|c|c|c|c|c|c|c|}
\hline \multirow{2}{*}{ Laminen-Typen } & \multirow{2}{*}{$\mathrm{Nr}$. } & \multirow{2}{*}{$\begin{array}{c}\text { Mächtig } \\
\text {-keit }\end{array}$} & \multicolumn{8}{|c|}{ Mikrofazieszonen } & \multirow{2}{*}{ Interpretation } \\
\hline & & & $1 a$ & $2 a$ & $2 b$ & $3 a$ & $3 b$ & $3 c$ & $3 d$ & $3 e$ & \\
\hline $\begin{array}{l}\text { Homogene quarzsandreiche } \\
\text { Ton/Siltlagen } \\
\text { Anhang 2-1, Fig. } 01 \text { u. Fig. } 02\end{array}$ & $1 a-1$ & $<1 \mathrm{~cm}$ & & & & & & & & & $\begin{array}{l}\text { Kontinuierlicher Eintrag von } \\
\text { mineralischem Feindetritus }\end{array}$ \\
\hline $\begin{array}{l}\text { Homogene quarzkornführende } \\
\text { Pyritlagen } \\
\text { Anhang 2-1, Fig. } 07\end{array}$ & $2 a-1$ & $<1 \mathrm{~cm}$ & & & & & & & & & $\begin{array}{l}\text { Kontinuierlicher Eintrag von } \\
\text { mineralischem Feindetritus } \\
\text { (primäre Schichtgrenzen durch } \\
\text { synsedimentäre Sulfid-Bildung } \\
\text { überprägt) }\end{array}$ \\
\hline $\begin{array}{l}\text { Lagen von matrixgestütztem } \\
\text { Quarzsand und -silt } \\
\text { Anhang 2-1, Fig. } 04\end{array}$ & $1 a-4,2 a-2$ & $\begin{array}{l}0,1 \mathrm{~mm}- \\
1 \mathrm{~mm}\end{array}$ & & & & & & & & & $\begin{array}{l}\text { Diskontinuierlicher Eintrag von } \\
\text { mineralischem Feindetritus }\end{array}$ \\
\hline $\begin{array}{l}\text { Quarzsandführende Siltlagen } \\
\text { Anhang 2-1, Fig. } 05 \text { u. Fig. } 06\end{array}$ & $1 a-2$ & $\begin{array}{l}0,2 \mathrm{~mm}- \\
1,5 \mathrm{~mm}\end{array}$ & & & & & & & & & Ablagerungen aus einer tem- \\
\hline $\begin{array}{l}\text { Quarzsandführende Ton/Silt- } \\
\text { lagen } \\
\text { Anhang 2-1, Fig. } 05 \text { u. Fig. } 06\end{array}$ & $1 a-3$ & $\begin{array}{l}0,2 \mathrm{~mm}- \\
1,5 \mathrm{~mm}\end{array}$ & & & & & & & & & $\begin{array}{l}\text { porär geschichteten Wasser- } \\
\text { säule (teils "Interflow"-Ablage- } \\
\text { rungen) }\end{array}$ \\
\hline $\begin{array}{l}\text { Lagen von korngestütztem } \\
\text { Quarzsand und -silt (extrakla- } \\
\text { stenführend, meist ungradiert) } \\
\text { Anhang 2-1, Fig. } 03 \text { bzw. } \\
\text { Anhang 2-2, Fig. } 01 \text { bis Fig. } 04\end{array}$ & $\begin{array}{l}1 a-5,2 a-3 \\
2 b-3,3 a-2\end{array}$ & $<1,5 \mathrm{~mm}$ & & & & & & & & & $\begin{array}{l}\text { Ablagerungen distaler "turbidity } \\
\text { currents" (Typ-I und Typ-II, vgl. }\end{array}$ \\
\hline $\begin{array}{l}\text { Sandführende Lagen von korn- } \\
\text { gestütztem Quarzsilt } \\
\text { Anhang 2-2, Fig. } 01 \text { bis Fig. } 04\end{array}$ & $2 b-2$ & $<50 \mu \mathrm{m}$ & & & & & & & & & \\
\hline $\begin{array}{l}\text { Extra- und Intraklastenführende } \\
\text { Tonlagen (ungradiert) } \\
\text { Anhang 2-2, Fig. } 06 \text { u. Fig. } 07 \\
\text { bzw. Anhang 2-3, Fig. } 07 \text { bzw. } \\
\text { Anhang 2-4, Fig. } 04\end{array}$ & $\begin{array}{c}3 a-3,3 b-3 \\
3 c-3,3 d-3 \\
3 e-3\end{array}$ & $<1 \mathrm{~mm}$ & & & & & & & & & $\begin{array}{l}\text { Ablagerungen distaler "turbidity } \\
\text { currents" (Typ-III, vgl. Tab. 30) }\end{array}$ \\
\hline $\begin{array}{l}\text { Organisch-reiche Tonlagen } \\
\text { vgl. Anhang } 2-1 \text { bis } 2-6\end{array}$ & $\begin{array}{c}2 b-1,3 a-1 \\
3 b-2,3 c-2 \\
3 d-2\end{array}$ & $\begin{array}{l}15 \mu \mathrm{m}- \\
3 \mathrm{~mm}\end{array}$ & & & & & & & & & $\begin{array}{l}\text { Weitgehend kontinuierlicher } \\
\text { Eintrag von allochthonem und } \\
\text { autochthonem POM sowie } \\
\text { mineralischem Feindetritus }\end{array}$ \\
\hline $\begin{array}{l}\text { Diatomeenlagen } \\
\text { Anhang 2-2, Fig. } 07 \text { u. Fig. } 08 \\
\text { bzw. Anhang 2-3, Fig. } 01 \text { bis } \\
\text { Fig. } 06\end{array}$ & $3 b-1$ & $\begin{array}{l}30 \mu \mathrm{m}- \\
1,5 \mathrm{~mm}\end{array}$ & & & & & & & & & Diatomeen-Blüten \\
\hline $\begin{array}{l}\text { Organisch-reiche Opallagen } \\
\text { Anhang 2-4, Fig. } 01 \text { bis Fig. } 05\end{array}$ & $3 c-1$ & $\begin{array}{l}5 \mu \mathrm{m}- \\
2 \mathrm{~mm}\end{array}$ & & & & & & & & & $\begin{array}{l}\text { Synsedimentäre Lösung von } \\
\text { Skelettopal }\end{array}$ \\
\hline $\begin{array}{l}\text { Diatomeen/Opal(CT)-Lagen } \\
\text { Anhang 2-5, Fig. } 01 \text { bis Fig. } 08 \\
\text { bzw. Anhang 2-6, Fig. } 01 \text { bis } \\
\text { Fig. } 04\end{array}$ & $3 d-1$ & $\begin{array}{l}10 \mu \mathrm{m}- \\
0,25 \mathrm{~mm}\end{array}$ & & & & & & & & & $\begin{array}{l}\text { Diatomeen-Blüten } \\
\text { (primäre Schichtgrenzen durch } \\
\text { frühdiagenetische Kieselsäure- } \\
\text { Migration teils überprägt) } \\
\end{array}$ \\
\hline $\begin{array}{l}\text { Opal(CT)-reiche Horizonte } \\
\text { Anhang 2-6, Fig. } 05 \text { bis Fig. } 08\end{array}$ & $3 e-1$ & $<0,5 \mathrm{~mm}$ & & & & & & & & & Primäre Schichtgrenzen durch \\
\hline $\begin{array}{l}\text { Tonreiche Horizonte } \\
\text { Anhang 2-6, Fig. } 07 \text { u. Fig. } 08\end{array}$ & $3 e-2$ & $<0,1 \mathrm{~mm}$ & & & & & & & & & $\begin{array}{l}\text { frundiagenetıscne Kleseisaure- } \\
\text { Migration überprägt }\end{array}$ \\
\hline $\begin{array}{l}\text { Siderit fein verteilt bzw. in } \\
\text { Linsen und Lagen angereichert } \\
\text { Anhang 2-2, Fig. } 05 \text { bzw. } \\
\text { Anhang 2-3, Fig. } 02 \text { u. Fig. } 03 \\
\text { bzw. Anhang 2-4, Fig. } 06 \text { u. Fig. } \\
07 \text { bzw. Anhang 2-5, Fig. } 04 \text { bis } \\
\text { Fig. } 06\end{array}$ & $\begin{array}{c}3 a-1,3 b-1 \\
3 c-4,3 d-4 \\
3 e-4\end{array}$ & $\begin{array}{l}10 \mu \mathrm{m}- \\
0,3 \mathrm{~mm}\end{array}$ & & & & & & & & & $\begin{array}{l}\text { Frühdiagenetische Siderit- } \\
\text { Bildung }\end{array}$ \\
\hline
\end{tabular}

Tab. 29: Beschreibung, Vorkommen und Deutung von Laminen-Typen innerhalb der profundalen Seesedimente 


\begin{tabular}{|c|c|c|c|c|c|c|}
\hline \multirow[b]{2}{*}{ Textur-Abschnitt } & & \multirow[b]{2}{*}{ Mächtigkeit } & \multicolumn{3}{|c|}{ Resedimente } & \multirow[b]{2}{*}{ Interpretation } \\
\hline & & & $\stackrel{\bar{d}}{\grave{1}}$ & 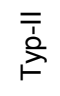 & 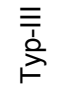 & \\
\hline $\begin{array}{l}\text { Regellos orientierte, komponenten- und matrixge- } \\
\text { stützte Extraklasten bis Steinkorngröße (im oberen } \\
\text { Textur-Abschnitt normalgradiert) } \\
\text { Anhang 2-7, Fig. } 01 \text { u. Fig. } 05 \text { bzw. Anhang 2-8, } \\
\text { Fig. } 01 \text { u. Fig. } 02\end{array}$ & $\mathrm{X}_{1}$ & bis $2,4 \mathrm{~m}$ & & & & $\begin{array}{c}\text { Ablagerungen von } \\
\text { "hyperconcentrated density flows" }\end{array}$ \\
\hline $\begin{array}{l}\text { Brekziierte, intern gefaltete Laminite und umgela- } \\
\text { gerte Resedimente ("slumping-" und "sliding"- } \\
\text { Strukturen) } \\
\text { Anhang 2-8, Fig. } 02 \text { u. Fig. } 03\end{array}$ & $\mathrm{X}_{2}$ & bis $1,4 \mathrm{~m}$ & & & & $\begin{array}{c}\text { Ablagerungen von } \\
\text { subaquatischen "slumps/slides" }\end{array}$ \\
\hline $\begin{array}{l}\text { Komponentengestützte Extraklasten bis Feinkies- } \\
\text { korngröße (normalgradiert, bei Typ-II-Resedimen- } \\
\text { ten mit eingeregelten Phytoklasten im oberen } \\
\text { Textur-Abschnitt) } \\
\text { Anhang 2-7, Fig. 01, Fig. } 02 \text { u. Fig. } 05 \text { bzw. Anhang } \\
\text { 2-8, Fig. } 04\end{array}$ & $\mathrm{Y}_{1}$ & bis $20 \mathrm{~cm}$ & & & & $\begin{array}{c}\text { Ablagerungen von } \\
\text { "concentrated density flows" }\end{array}$ \\
\hline $\begin{array}{l}\text { Eingeregelte Laminen-Fragmente und Phytoklasten } \\
\text { (normalgradiert, im oberen Textur-Abschnitt gele- } \\
\text { gentlich Anreicherung von Quarzsand) } \\
\text { Anhang 2-8, Fig. } 04\end{array}$ & $\mathrm{Y}_{2}$ & bis $60 \mathrm{~cm}$ & & & & Ablagerungen von \\
\hline $\begin{array}{l}\text { Korngestützte Quarze bis Feinsandkorngröße (bei } \\
\text { Typ-II- und Typ-III-Resedimenten normalgradiert) } \\
\text { Anhang 2-7, Fig. } 01 \text { bis Fig. } 03 \text { u. Fig. } 05\end{array}$ & Z & bis $15 \mathrm{~cm}$ & & & & \\
\hline
\end{tabular}

Tab. 30: Beschreibung, Vorkommen und Deutung von Textur-Abschnitten innerhalb idealer ResedimentSequenzen der profundalen Seesedimente

streng saisonal gesteuerten Wechsel von Stagnation und Zirkulation im Eckfeld-See unwahrscheinlich. Es ist vielmehr davon auszugehen, daß die Turbulenzen häufiger, teils hochkonzentrierter Sedimentströme sporadisch zu einer vertikalen Durchmischung des Wasserkörpers beitrugen. Insgesamt deuten die sedimentologischen Befunde auf einen oligotrophen, warm polymiktischen See mit irregulärem Zirkulationsmuster hin.

\section{Lithofazieszone 2: Übergangsschichten}

Teufe:

E1/96: $37,67 \mathrm{~m}$ bis $33,15 \mathrm{~m}$

E3/96: $36,79 m$ bis $32,65 m$

Die LFZ 2: Übergangsschichten ist durch das Einsetzen der organischen Sedimentation im Eckfelder Maar gekennzeichnet. Extraklastenreiche Resedimente (Typ-II-Resedimente, vgl. Tab. 30) bleiben jedoch ein signifikanter Bestandteil der Profilsequenz.

Innerhalb der basalen MFZ 2a: Quarz-Pyrit-Fazies tritt Pyrit mit Werten zwischen rund 48 Vol.\% und über 85 Vol.\% gesteinsbildend auf. Der ungewöhnlich hohe Pyritisierungsgrad ist mit dem stratigraphisch ersten Nachweis von Primärproduzenten (Botryococcus cf. braunii) und organischem Detritus (hauptsächlich Texto-Ulminit und Humodetrinit) in den Eckfelder Profundalsedimenten korreliert. Offensichtlich löste der Eintrag von degradierbarer organischer Substanz als Reduktionsmittel und Energiequelle eine intensive bakterielle Reduktion eines angestauten Sulfat-Reservoirs aus. In den pyritreichen Sedimenten der MFZ 2a: Quarz-Pyrit- 
Fazies ist der Beginn einer meromiktischen Seephase dokumentiert. Neben der Akkumulation von Schwefelwasserstoff und Hydrogenkarbonat aus der bakteriellen Sulfatreduktion trug insbesondere die Anreicherung von reduziertem Eisen im anoxischen Tiefenwasser zu einer Stabilisierung der Chemokline bei. Verbunden mit dem kontinuierlich zunehmenden Eintrag von organischer Substanz führte die permanente Trennung eines stagnierenden, anoxischen Monimolimnions von einem zirkulierenden oxischen Mixolimnion zur Ablagerung der organischreichen Laminite im Hangenden der Lithofazieszone 2.

In der folgenden MFZ 2b: Quarz-Huminit-Fazies wechsellagern geringmächtige, siliziklastische Turbidite mit bis $0,5 \mathrm{~mm}$ mächtigen organisch-reichen Tonlagen. Die Dominanz von huminitischem Detritus innerhalb der tonreichen Horizonte markiert eine allmähliche Besiedelung der Kraterrandbereiche durch Landpflanzen. Gleichzeitig steigen auch die Gehalte Sporomorphen (Sporinit) im Sediment.

\section{Lithofazieszone 3: Biogene Laminite}

Teufe:

E1/96: $33,15 \mathrm{~m}$ bis $5,00 \mathrm{~m}$

E2/96: 20,50m (Endteufe) bis 4,50m

E3/96: $32,65 \mathrm{~m}$ bis $4,25 \mathrm{~m}$

Die für die liegende Mikrofazieszone typischen Quarzkornlagen verlieren innerhalb der MFZ 3a: Huminit-Fazies zunehmend an Bedeutung. Mit der Abnahme siliziklastischer Einschaltungen geht ein deutlicher Anstieg der POM-Werte von durchschnittlich 9.5 Vol.\% in MFZ $2 \mathrm{~b}$ auf 29,5 Vol.\% in MFZ 3a einher. Neben den erhöhten Anteilen von organischen Partikeln im Sediment ist dieser Profilabschnitt auch durch das erstmalige Auftreten von frühdiagenetisch gebildetem Siderit in Form von maximal 300 mm mächtigen Horizonten oder isolierten Linsen gekennzeichnet. Das gemeinsame Auftreten von Siderit und Pyrit im Sediment spricht für anoxisch-nichtsulfidisch-methanische Milieubedingungen. Unter diesen geochemischen Verhältnissen sind die Voraussetzungen für eine Sideritgenese erst nach der weitgehenden Konsumierung von $\mathrm{HS}^{-}$durch die Reaktion mit detritischen Eisenmineralen gegeben.

Die MFZ 3b: Aulacoseira-Fazies beginnt mit dem ersten mikroskopischen Nachweis von Diatomeen-Frusteln bei Bohrmeter 31,80 (E1/96) bzw. 31,38 (E3/96). Im Vergleich zu anderen lakustrinen Tertiärvorkommen Mitteleuropas, wie beispielsweise Sieblos/Rhön (Unteroligozän), Enspel/Westerwald (Oberoligozän) und Rott/Siebengebirge (Oberoligozän), zeichnet sich die Diatomeen-Flora von Eckfeld durch eine ungewöhnlich geringe Diversität aus. Sie wird weitgehend durch den Indiviuenreichtum von Aulacoseira cf. granulata geprägt. Die zentrischen Kieselalgen treten in $30 \mu \mathrm{m}$ bis $1,5 \mathrm{~mm}$ mächtigen Lagen konzentriert auf und bilden mit maximal 25 Vol.\% einen Hauptgemengteil des Gesteins. Nach den ökologischen Anforderungen der rezenten Art ist auf einen eutrophen, Si-reichen See mit leicht alkalischem (etwa im Bereich von $\mathrm{pH}$ 8) Mixolimnion zu schließen. Die Anreicherungen von Aulacoseira-Schalen in nahezu monospezifischen Lagen belegen episodische Massenentfaltungen der Kieselalge, die vermutlich durch den Wechsel von Niederschlags- und Evaporationsphasen unter tropischen bis subtropischen Klimabedingungen gesteuert wurden. 
Die MFZ 3b: Aulacoseira-Fazies schließt zum Hangenden hin mit einem Typ-III-Resediment (vgl. Tab. 30) ab. Es beinhaltet ein ungeordnetes Gemenge aus zerscherten und intern gefalteten Laminit-Bruchstücken. Mit 2,65m stellt diese Rutschungsbrekzie die mächtigste Resediment-Sequenz innerhalb der untersuchten Profilabfolge dar. Sie ist in ähnlicher Mächtigkeit ebenfalls aus der rund 60m südwestlich gelegenen Bohrung von 1980 bekannt. Der Rutschkörper breitete sich offensichtlich über weite Teile des zentralen Seebodens aus.

Auffällig ist der fehlende Nachweis von Phytoplankton-Vertretern (Botryococcus cf. braunii und Aulacoseira cf. granulata) direkt im Hangenden des Resediments (MFZ 3c: Huminit-OpalFazies). Die von der Massenverlagerung induzierten Turbulenzen hatten sehr wahrscheinlich eine partielle oder vollständige Auflösung der Fe(II)hydrogenkarbonat-Meromixis im EckfeldSee und ein Aufdringen toxischer Verbindungen, wie $\mathrm{H}_{2} \mathrm{~S}$ und $\mathrm{CH}_{4}$, in die photische Zone zur Folge. Während Botryococcus im oberen Teil der Sedimentfolge erneut einsetzt, bleibt der Nachweis von Diatomeen aus. Ob Letzteres tatsächlich temporär ungünstige Lebensbedingungen für Aulacoseira widerspiegelt, kann nicht abschließend geklärt werden. So deuten ausgeprägte Korrosionserscheinungen an der einzigen innerhalb der MFZ 3c nachgewiesenen Chrysophyceen-Zyste auf eine verstärkte Lösung von biogenem Opal bereits im Bereich der Sediment/Wassergrenze hin. Vermutlich führte die Mischung mit alkalischen Oberflächenwasser (siehe oben) zu höheren $\mathrm{pH}-$ Werten im Tiefenwasser.

Die folgende MFZ 3d: Aulacoseira-Opal(CT)-Fazies ist durch das erneute Auftreten von Diatomeen-Resten in $10 \mu \mathrm{m}$ bis $250 \mu \mathrm{m}$ mächtigen Lagen gekennzeichnet. Im Gegensatz zu den diagenetisch unveränderten Diatomeenlagen der MFZ 3b: Aulacoseira-Fazies zeichnen sich die diatomeenreichen Horizonte in diesem Profilabschnitt jedoch durch eine fortgeschrittene Umwandlung von Skelettopal (Opal-A) zu Opal-CT aus. Opal-CT wurde in Form von maximal $3 \mu \mathrm{m}$ großen sphärischen Aggregaten (Lepisphären) vorwiegend in den Hohlräumen gelöster Diatomeen-Schalen nachgewiesen. Die Kieselsäure-Migration hatte eine Überprägung der primären Schichtgrenzen zwischen opal- und tonreichen Lagen zu Folge.

Im Profilbereich der MFZ 3e: Tetraedron-Opal(CT)-Fazies sind die Gehäusereste von Aulacoseira cf. granulata nur noch vereinzelt und lediglich an der Basis der Abfolge nachzuweisen. Daß Diatomeen dennoch eine bedeutende Phytoplankton-Gruppe im Eckfeld-See darstellten ist durch häufige Schalenabdrücke im opalreichen Sediment belegt. Für diese Phase der See-Entwicklung ist also weiterhin von einem eutrophen, leicht alkalischen Mixolimnion auszugehen. Das teils individuenreiche Auftreten der Grünalge Tetraedron cf. minimum in MFZ 3e widerspricht dieser Vorstellung nicht, da es sich bei den Vertretern der Gattung Tetraedron generell um eutrophe Arten zu handeln scheint. Der fluoreszenzmikroskopische Nachweis von Tetraedron ist in Eckfeld allerdings maßgeblich an den Verkieselungsgrad der Sedimente gebunden. Während die Grünalge in schwächer opalisierten Tonhorizonten stark kompaktiert wurde, blieben die dünnwandigen, $8 \mu \mathrm{m}$ bis $11 \mu \mathrm{m}$ großen Einzelzellen in opalreichen Sedimentabschnitten nahezu vollkörperlich erhalten. Dieser Umstand ist ein starker Hinweis auf eine äußerst frühdiagenetische Lösung und Wiederausfällung biogener Kieselsäure. Die Umwandlung von Skelettopal zu Opal-CT vollzog sich offensichtlich vor einer weitreichenden Sedimentkonsolidierung. Eine dreidimensionale Überlieferung von Tetraedron wäre andernfalls kaum zu erklären. 
Im Gegensatz zu den devonklastenreichen Resedimenten (Typ-I und Typ-II, vgl. Tab. 30) zeichnen sich die Typ-III-Resedimente der LFZ 3 durch schichtparallel eingelagerte LaminitFragmente als bedeutende Gesteinskomponente aus. Die hohen Gehalte an Intraklasten belegen, daß die Bildung organisch-reicher Pelite nicht nur auf die zentralen Seebereiche beschränkt war. Offensichtlich lagen weite Teile der Kraterhänge innerhalb des anoxischen Monimolimnions. Eine Höhenlage der Chemokline oberhalb von Regionen, deren Hangneigung für die Entstehung von Massenverlagerungen ausreichte, erklärt den allgemeinen Reichtum an umgelagerten Schwarzpeliten innerhalb der Lithofazieszone 3: Biogene Laminite.

Aus organisch-petrologischer Sicht ist in den profundalen Seeablagerungen von Eckfeld ein deutlicher Trend von einer vorwiegend allochthonen hin zu einer stärker autochthon geprägten organischen Sedimentation entwickelt. Der rückläufige Nachweis von huminitischem Detritus ab der MFZ 3c: Huminit-Opal-Fazies geht mit einer generell abnehmenden Häufigkeit siliziklastischer Schüttungsereignisse einher. Zu diesem Zeitpunkt der See-Entwicklung waren die Kraterwände offensichtlich weitgehend stabilisiert. Das Spektrum der überlieferten Primärproduzenten spricht für einen permanent eutrophen See. Lediglich die mineralischen Sedimente der MFZ 1a: Quarz-Fazies an der Basis der untersuchten Profilabfolge wurden in einem oligotrophen Wasserkörper abgelagert.

Die mikrofaziellen und organisch-petrologischen Untersuchungen ergaben keine Hinweise auf ein jahreszeitlich induziertes Sedimentationsmuster im ehemaligen Eckfeld-See. Vielmehr scheinen die Alternationen aus Diatomeenlagen und organisch-reichen Tonhorizonten innerhalb der MFZ 3b: Aulacoseira-Fazies, eine supersaisonale Zyklizität im Wechsel von regenreichen und regenarmen Jahren abzubilden. 


\section{Literatur}

Aaronson, S., Berner, T., Gold, K., Kushner, L., Patni, N.J., Repak, A. \& Rubin, D. (1983): Some observations on the green planktonic alga Botryococcus braunii and its bloom form. $-\mathrm{J}$. Plankton Research, 5: 693-700.

AlLmAN, M. \& LAWRENCE, D.F (1972): Geological Laboratory Techniques. - Blandford Press, London, $335 \mathrm{~S}$

ALPERN, B. (1981): Les schistes bitumineux: constitution, réserves, valorisation. - Bulletin du Centres Recherche Exploration-Production Elf-Aquitaine, 5: 319-352.

BAHRIG, B. (1988): Palaeo-environment information from deep water siderite (Lake of Laach, West Germany). - In: FLEET, A.J. et al. [eds.]: Lacustrine Petroleum Source Rocks. - Geol. Soc. Spec. Publ., 40: 153-158.

BAHRIG, B. (1989): Stable isotope composition of siderite as an indikator of the paleoenvironmental history of oil shale lakes. - Palaeogeogr., Palaeoclimat., Palaeoecol., 70: 139-151.

BARKER, P. (1992): Differential diatom dissolution in Late Quaternary sediments from Lake Manyara, Tanzania: an experimental approach. - J. Paleolimnol., 7: 235-251.

BARKER, P., FONTES, J.-C., GASSE, F. \& DRUART, J.-C. (1994): Experimental dissolution of diatom silica in concentrated salt solutions and implications for paleoenvironmental reconstroction. - Limnol. Oceanogr., 39(1): 99-110.

BATTEN, D.J. (1996a): Palynofacies and palaeoenvironmental interpretation. - In: JANSONIUS, J. \& McGREGoR, D.C. [eds.]: Palynology: Principles and Applications, vol. 3 - New Directions, other Applications and Floral History. - Am. Assoc. Strat. Palyn. Found.: 1011-1064.

BATTEN, D.J. (1996b): Colonial Chlorococcales. - In: JANSONIUS, J. \& MCGREGOR, D.C. [eds.]: Palynology: Principles and Applications, vol. 1 - Principles. - Am. Assoc. Strat. Palyn. Found.: 191203.

BATTEN, D.J. \& GRENFELL, H.R. (1996): Botryococcus. - In: JANSONIUS, J. \& MCGREGOR, D.C. [eds.]: Palynology: Principles and Applications, vol. 1 - Principles. - Am. Assoc. Strat. Palyn. Found.: 205214.

Behrensmeyer, A.K., DAmUth, J.D., DiMichele, W.A., PotTs, R., Sues, H.-D. \& Wing, S.L. (1992): Terrestrial Ecosystems through Time. - Univ. Chicago Press, Chicago and London, $568 \mathrm{~S}$.

Berkaloff, C., Casadevall, E., Largeau, C., Metzger, P., Peracca, S. \& Virlet, J. (1983): The resistant polymer of the walls of the hydrocarbon-rich alga Botryococcus braunii. - Phycochemistry, 22: 389-397.

BERNER, R.A. (1969): The synthesis of framboidal pyrite. - Econ. Geol., 64: 383-384.

BERnER, R.A. (1971): Principles of Chemical Sedimentology. - McGraw-Hill, New York, 240 S.

BERNER, R.A. (1981a): A new Geochemical Classification of Sedimentary Environments. - J. Sed. Petrol., 51(2): 359-365.

BERNER, R.A. (1981b): Authigenic mineral formation resulting from organic matter decomposition in modern sediments. - Fortschr. Miner., 59: 117-135. 
BERnER, R.A. (1984): Sedimentary pyrite formation: An update. - Geochim. et Cosmochim. Acta, 48: 605615.

Blokker, P., Schouten, S., van den Ende, H., De LeeuW, J.W., Hatcher, P.G. \& Sinninghe Damste, J.S. (1998): Chemical structure of algaenans from the fresh water algae Tetraedron minimum, Scenedesmus communis and Pediastrum boryanum. - Org. Geochem., 29: 1453-1468.

BoumA, A.H. (1962): Sedimentology of Some Flysch Deposits. - Elsevier, Amsterdam, 168 S.

BRAUER, J. \& LUTZ, H. (1990): Forschungsbericht Grabung Eckfeld. - Mitt. Rhein. Naturforsch. Ges., 11: 13-23.

BRAUN, A. (2001): Ein phosphatisierter Süßwasser-Schwamm aus dem Tertiär von Rott (Siebengebirge, Rheinland). - Paläont. Zeitschr., 75(1): 43-50.

BüCHEL, G. (1992): Das Kelberger Hoch. - Die Geowissenschaften, 10/5: 133-142.

BÜCHEL, G. (1993): Maars of the Westeifel, Germany. - In: NegENDANK, J. F. W. \& ZoLITSCHKA, B. [eds.]: Paleolimnology of European Maar Lakes. - Lekture Notes in Earth Sciences, 49: 1-13.

BÜCHEL, G. \& LORENZ, V. (1993): Syn- and post-eruptive mechanism of the Alaskan Ukinrek Maars in 1977. - In: NegendANK, J. F. W. \& ZolitschKA, B. [eds.]: Paleolimnology of European Maar Lakes. Lekture Notes in Earth Sciences, 49: 15-60.

BULLWINKEL, V. \& RIEGEL, W. (2000): Organic matter distribution in sediments of the Eckfeld maar lake. Terra Nostra 2000/6: International Maar Conference, Daun/Vulkaneifel: 83-85.

BULLWINKEL, V. \& RIEGEL, W. (2001): The Laminated Lake Sediments of the Eckfeld Maar (Middle Eocene, Germany): Types of Stratification and Role of Organic Matter. - Facies, 45: 165-176.

BULLWINKEL, V. \& SosNITZA, M. (2000): Eine Methode zur Herstellung von Dünnschliffen aus gering verfestigten, ungetrockneten Schwarzpeliten. - Mainzer naturwiss. Archiv, 38: 1-10.

Burgis, M.J. \& MoRRIS, P. (1987): The Natural History of Lakes. - Cambridge Univ. Press, Cambridge, $218 \mathrm{~S}$.

Bustin, R.M., Cameron, A.R., Grieve, D.A. \& Kalkreuth, W.D. (1985): Coal Petrology: Its Principles, Methods, and Applications. - Geol. Assoc. Canada, Short Course Notes: 3, St. Johns, 230 S.

CAnfield, D.E. \& Raiswell, R. (1991): Pyrite formation and fossil preservation. - In: AlLison, P.A. \& BRIGGS, D.E.G. [eds.]: Taphonomy: releasing the data locked in the fossil record. - Plenum Press, New York: 337-386.

CANTAREL, P. \& Lippold, H. J. (1977): Alter und Abfolge des Vulkanismus der Hocheifel. - Jb. Geol. Paläont., Mh. 1977: 600-612.

Chacón-Baca, E., Beraldi-Campesi, H., Cevallos-Ferriz, S.R.S., Knoll, A.H. \& GolubiC, S. (2002): 70 Ma nonmarine diatoms from nothern Mexico. - Geology, 30(3): 279-281.

Chaffee, A.L., Johns, R.B., BAeRKen, M.J., LeeuW, J.W., SchenK, P.A. \& Boon, J.J. (1984): Chemical effects in gelification processes and lithotype formation in Victorian brown coals. - Adv. Org. Geochem., 6: 409-416.

CHOLNOKY, B.J. (1968): Die Ökologie der Diatomeen in Binnengewässern. - J. Cramer, Lehre, 699 S.

ClAUSING, A. (1998): Mikro-organofazielle Studien an Sedimenten des Enspel-Sees (Oberoligozän, Westerwald, Deutschland). - Hallesches Jahrb. Geowiss., B20: 119-133. 
CLAUSING, A. (2000): Limnische Primärproduktion im deutschen Tertiär: Kenntnisstand und Perspektiven. - Zbl. Geol. Paläont. Teil II, 1/2: 1-13.

CLAUSING, A. (2001): Primärproduktion und Laminationsbildung ausgewählter lakustriner Environments im Tertiär und Permokarbon Deutschlands. - Hallesches Jahrb. Geowiss., Reihe B, Beiheft 14: 1-183.

COHEN, A.D., SPACKMAN, W. \& RAYMOND, R. (1987): Interpreting the characteristics of coal seams from chemical, physical and petrographic studies of peat deposits. - in: ScotT, A.C. [ed.]: Coal and Coalbearing Strata: Recent Advances. - Geol. Soc. Spec. Publ., 32: 107-125.

Cole, R.D. \& PICARD, M.D. (1975): Primary and Secondary Sedimentary Structures in Oil Shale and other Fine-grained rocks, Green River Formation (Eocene), Utah and Colorado. - Utah Geol., 2/1: 49-67.

ColLINSON, J.D. \& THOMPSON, D.B. (1989): Sedimentary Structures. - Unwin \& Hyman, London, 207 S.

Cook, A.C. (1982): Organic Petrology. - In: CoOK, A.C. [ed.]: The origin and petrology of organic matter in coals, oil shales and petroleum source-rocks. - Univ. Wollongong, Australia: 1-35.

COOK, A.C. \& KANTSLER, A.J. (1982): Description of the maceral groups and macerals found in coals, oil shales, and as dispersed organic matter in sediments. - In: COOK, A.C. [ed.]: The origin and petrology of organic matter in coals, oil shales and petroleum source-rocks. - Univ. Wollongong, Australia: 3665.

CRAWFORD, R.M. (1975): The taxonomy and classification of the diatom genus Melosira C. Ag., I. The type species M. nummuloides C. Ag. - Br. phycol. J., 10: 323-338.

CROSSLEY, R. \& OWEN, B. (1988): Sand turbidites and organic-rich diatomaceous muds from Lake Malawi, Central Africa. - In: FLEET, A.J. et al. [eds.]: Lacustrine Petroleum Source Rocks. - Geol. Soc. Spec. Publ., 40: 369-374.

DAMNATI, B., TAIEB, M. \& WILLIAMSON, D. (1992): Laminated deposits from Lake Magadi (Kenya). Climate contrast effect during the maximum wet period between 12,000-10,000 yrs B.P. - Bull. Soc. géol. France, 163: 407-414.

DANNAT, C. (2000): Forschungsprojekt Eckfelder Maar: Jahresbericht 1999. - Mitt. Rhein. Naturforsch. Ges., 21: 17-21.

DAVISON, W. (1988): Interactions of iron, carbon and sulphur in marine and lacustrine sediments. - In: FLEET, A.J. et al. [eds.]: Lacustrine Petroleum Source Rocks. - Geol. Soc. Spec. Publ., 40: 131-137.

DECKER, K. (1991): Rhythmic Bedding in Siliceous Sediments - an Overview. - In: EINSELE, G. et al. [eds.]: Cycles and Events in Statigraphy. - Springer, Berlin usw.: 464-479.

DE DECKKER, P. (1988): Large Australian lakes during the last 20 million years: sites for petroleum source rock or metal ore deposition, or both? - In: FLEET, A.J. et al. [eds.]: Lacustrine Petroleum Source Rocks. - Geol. Soc., Spec. Publ., 40: 45-58.

Dehm, R., Gall, H., Höfling, R., Jung, W. \& Malz, H. (1977): Die Tier- und Pflanzenreste aus den obermiozänen Riessee-Ablagerungen in der Forschungsbohrung Nördlingen 1973. - Geologica Bavarica, 75: 91-109.

DELLINO, P. (2000): Phreatomagmatic deposits: fragmentation, transportation and deposition mechanisms. - Terra Nostra 2000/6: International Maar Conference, Daun/Vulkaneifel: 99-105.

DEN BRoK, B. \& Hofmeister, W. (2000): Palagonites from the Eckfeld Maar and the Hillscheider Diatrem, Hocheifel, Germany. - Terra Nostra 2000/6: International Maar Conference, Daun/Vulkaneifel: 78-82. 
Derenne, S., Metzger, P., Largeau, C., Van Bergen, P.F., Gatellier, J.P., Sinninghe Damsté, J.S., de LEEUW, J.W. \& BERKALOFF, C. (1991): Similar morphological and chemical variations of Gloeocapsomorpha prisca in Ordovician sediments and cultured Botryococcus braunii as a response to changes in salinity. - In: ECKARDT, C.B. et al. [eds]: Advances in Organic Geochemistry 1991. Organic Geochem., 19: 299-313.

DIESSEL, C.F.K. (1992): Coal-Bearing Depositional Systems. - Springer, Berlin usw., 721 S.

Dodson, J.R. \& RAMRATH, A. (2001): An Upper Pliocene lacustrine environmental record from southWestern Australia - preliminary results. - Palaeogeogr., Palaeoclimat., Palaeoecol., 167: 309-320.

DUFF, K.E. \& SMOL, J.P. (1994): Chrysophycean cyst flora from British Columbia (Canada) lakes. - Nova Hedwigia, 58: 353-389.

DufF, K.E., ZEEB, B.A. \& SMOL, J.P. (1995): Atlas of Chrysophycean cysts. - Developments in Hydrobiology, 99. - Kluwer Academic Publishers, Dordrecht, 189 S.

EAton, R.A. \& Hale, M.D.C. (1993): Wood: Decay, Pests and Protection. - Chapman \& Hall, London, $546 \mathrm{~S}$.

EINSELE, G. (1991): Submarine Mass Flow Deposits and Turbidites. - In: EINSELE, G. et al. [eds.]: Cycles and Events in Statigraphy. - Springer, Berlin usw.: 313-339.

EMERSON, S. (1976): Early diagenesis in anaerobic lake Sediments: chemical equilibria in interstitial waters. - Geochim. et Cosmochim. Acta, 40: 925-934.

Enos, P. (1973): Channelized submarine carbonate debris flow, Cretaceous, Mexico. - Bull. Am. Assoc. Petrol. Geol., 57: 777.

ENOS, P. (1977): Flow regimes in debris flow. - Sedimentology, 24: 133-142.

ERIKsson, K.-E-L., Blanchette, R.A. \& Ander, P. (1990): Microbial and Enzymatic Degradation of Wood and Wood Components. - Springer, Berlin usw., $407 \mathrm{~S}$.

FABRICIUS, F. (1962): Die Strukturen des "Rogenpyrits" (Kössener Schichten, Rät) als Beitrag zum Problem der "vererzten Bakterien". - Geol. Rdsch., 51: 647-657.

FAHN, A. (1982): Plant Anatomy. - Pergamon Press, Oxford, 544 S.

FELDER , M. \& GAUPP, R. (2001): Paläolimnische Rekonstruktion paläogener Seen anhand authigen gefällter Siderite. - In: GAUPP, R. \& VAN DER KLAUW, S. [Hrsg.]: Sediment 2001 - Programm, Kurzfassungen, Exkursionsführer. - Schriftr. Dtsch. Geol. Ges., 13: 35.

Felder, M., Gaupp, R. \& WutTKE, M. (2000a): Sideritgenese in lakustrinen Sedimenten. - Mitt. Ges. Geol. Bergbaustud. Österr., 43: Sediment 2000, Kurzfassungen: 42.

FElder, M., GAupP, R. \& WUTTKE, M. (2000b): Siderite precipitation in Paleogene maar lakes of Central Europe. - Terra Nostra 2000/6: International Maar Conference, Daun/Vulkaneifel: 112-117.

FELDER, M. \& HARMS, F.-J. (2001): Forschungsbohrung Messel 2001, Profilbeschreibung. - http://home.tonline.de/home/brg_me_01/Profilbeschreibung.doc.

FELDER, M., WeidenfELLER, M. \& WUTTKE, M. (1998): Lithologische Beschreibung einer Forschungsbohrung im Zentrum des oberoligozänen, vulkano-lakustrinen Beckens von Enspel/Westerwald (Rheinland-Pfalz; Bundesrep. Deutschland). - Mainzer Geowiss. Mitt., 27: 101136. 
FISCHER, C. (1999): Grobklastika im mitteleozänen Eckfelder Maar (Südwesteifel): Sedimentologische und petrographische Analyse. - Mainzer naturwiss. Archiv, 37: 21-54.

FISCHER, C., GAUPP, R. \& PIRRUNG, M. (2000): Coarse grained sediments from the Eckfeld Maar (Middle Eocene, Eifel, Germany): Development of sedimentary facies. - Terra Nostra 2000/6: International Maar Conference, Daun/Vulkaneifel: 118-122.

FISHER, R.V. (1971): Features of coarse-grained, high-concentration fluids and their deposits. - J. Sed. Petrol., 41: 916-927.

FISHER, R.V. (1983): Flow transformations in sediment gravity flows. - Geology, 11: 273-274.

FLÖRKE, O.W., HOLLMANN, R., RAD, U. VON \& RÖSCH, H. (1976): Intergrowth and twinning in opal-CT lepispheres. - Contr. Mineral. Petrol., 58: 235-242.

Foged, N. (1980): Diatoms in Öland, Sweden. - Bibl. Phycol., 49: 193 S.

Foster, C.B., CERnOvskis, A. \& O’BRIEN, G.W. (1985): Organic-walled microfossils from the Early Cambrian of South Australia. - Alcheringa, 9: 259-268.

FOTT, B. (1971): Algenkunde. - VEB G. Fischer, Jena, 581 S.

FRANKENHÄUSER, H. \& WILDE, V. (1993): Flowers from the Middle Eocene of Eckfeld (Eifel, Germany) First results. - In: Negendank, J. F. W. \& ZolitschKA, B. [eds.]: Paleolimnology of European Maar Lakes. - Lekture Notes in Earth Sciences, 49: 491-497.

FRANKENHÄUSER, H., GwOSDEK, S. \& LUTZ, H. (1997): Forschungsprojekt Eckfelder Maar: 1996. - Mitt. Rhein. Naturforsch. Ges., 18: 41-45.

FRANZEN, J.L. (1993): Das biostratigraphische Alter der Fossillagerstätte Eckfelder Maar bei Manderscheid (Eifel). - Mainzer naturwiss. Archiv, 31: 201-214.

FRANZEN, J.L. (1994): Neue Säugerfunde aus dem Eozän des Eckfelder Maares bei Manderscheid (Eifel). - In: NeUfFER, F.O. et al. [Hrsg.]: Fossillagerstätte Eckfelder Maar. - Mainzer naturwiss. Archiv, Beiheft 16: 189-211.

FRANZEN, J.L. (1995): Die Equoidea des europäischen Mitteleozäns (Geiseltalium). - Hallesches Jahrb. Geowiss., B17: 31-45.

FÜCHTBAUER, H. (1988a): Kieselgesteine. - In: FüCHTBAUER, H. [Hrsg.]: Sedimente und Sedimentgesteine, Teil II: Sedimentpetrologie. - Schweizerbart, Stuttgart: 501-542.

FÜCHTBAUER, H. (1988b): Transportvorgänge und Sedimentstrukturen. - In: FÜCHTBAUER, H. [Hrsg.]: Sedimente und Sedimentgesteine, Teil II: Sedimentpetrologie. - Schweizerbart, Stuttgart: 779-863.

Galloway, W.E. \& HobDAY, D.K. (1998): Terrigenous Clastic Depositional Systems - Applications to Fossil Fuel and Groundwater Resources. - Springer, Berlin usw., 489 S.

GAUCH, H.G. (1982): Multivariate Analysis in Community Ecology. - Cambridge Univ. Press, Cambridge, 298 S.

GAYLORD, D.R., PRICE, S.M. \& SUYDAM, J.D. (2001): Volcanic and hydrothermal influences on middle Eocene lacustrine sedimentary deposits, Republic Basin, northern Washington, USA. - In: WHITE, J.D.L. \& RIGGS, N.R. [eds.]: Volcaniclastic Sedimentation in Lacustrine Settings. - Spec. Publs. int. Assoc. Sediment., 30: 199-222. 
Gelin, F., de LeeuW, J. W., Sinninghe Damsté, J. S., Derenne, S., Largeau, C. \& Metzger, P. (1994): Similarity of chemical structures of soluble aliphatic polyaldehyde and insoluble algaenan in the green microalga Botryococcus braunii race A as revealed by analytical pyrolysis. - Org. Geochem., 21: 423435.

GiResse, P., MALEY, J. \& KeltS, K. (1991): Sedimentation and palaeoenvironment in crater lake Barombi Mbo, Cameroon, during the last 25,000 years. - Sediment. Geol., 71: 151-175.

GlagoleV, A.A. (1934): Quantitative analysis with the microscope by the point method. - Eng. Min. J., 135: $399-400$.

GLENN, C.R. \& KeLTS, K. (1991): Sedimentary Rhythms in Lake Deposites. - In: EINSELE, G. et al. [eds.]: Cycles and Events in Statigraphy. - Springer, Berlin usw.: 188-221.

GómEZ FERNÁNDEZ, J.C. \& MELÉNDEZ, N. (1991): Rhythmically laminated lacustrine carbonates in the Lower Cretaceous of La Serranía de Cuenca Basin (Iberian Ranges, Spain). - In: ANADÓN, P. et al. [eds.]: Lacustrine Facies Analysis. - Spec. Publs. int. Assoc. Sedimentol., 13: 245-256.

GotH, K. (1990): Der Messeler Ölschiefer - Ein Algenlaminit. - Cour. Forsch.-Inst. Senckenberg, 131: 1143.

Goth, K., DE LeeuW, J.W., PüttmanN, W. \& TegelaAR, E.W. (1988): Origin of Messel Oil Shale kerogen. - Nature, 336: 759-761.

GROH, K. \& JUNGBLUTH, J.H. (1994): Vorläufige Mitteilung zur Najadenfauna (Mullusca: Bivalvia: Unionidae) des Eckfelder Maares (Mittel-Eozän). - In: NEUFFER, F.O. et al. [Hrsg.]: Fossillagerstätte Eckfelder Maar. - Mainzer naturwiss. Archiv, Beiheft 16: 151-165.

GRUBER, G. (1994): Kieselschwamm-Nadeln aus dem Mitteleozän des Eckfelder Maares, Eifel (Porifera: Demospongiae: Spongillidae). - Mainzer naturwiss. Archiv, 32: 1-6.

GRUBER, G. \& SchÄFER, P. (2000): Mitteleozäne Süßwasser-Ostracoden aus dem Eckfelder Maar (Vulkaneifel). - Mainzer naturwiss. Archiv, 38: 11-25.

GuY-OHLSON, D. (1992): Botryococcus as an aid in the interpretation of palaeoenvironment and depositional processes. - Rev. Palaeobot. Palynol., 71: 1-15.

GUY-OHLSON, D. (1998): The use of the microalga Botryococcus in the interpretation of lacustrine environments at the Jurassic-Cretaceous transition in Sweden. - Palaeogeogr., Palaeoclimat., Palaeoecol., 140: 347-356.

HALFMAN, J.D. \& JOHNSON, T.C. (1988): Lake Turkana - High-resolution record of cyclic change during the past $4 \mathrm{Ka}$ from lake Turkana, Kenya. - Geology, 16: 496-500.

HAMPTON, M.A. (1972): The role of subaqueous debris flow in generating turbidity currents. - J. Sed. Petrol., 42: 775-793.

HAMPTON, M.A. (1975): Competence of fine-grained debris flows. - J. Sed. Petrol., 45: 834-844.

HANSELMANN, , K. (1989): Rezente Sedimente - Lebensraum für Mikroorganismen. - Die Geowissenschaften, 7(4), 98-112.

harmon, M.E., Franklin, J.F., Swanson, F.J., Sollins, P., Gregory, S.V., Lattin, J.D., ANDERSON, N.H., Cline, S.P., Aumen, N.G., Sedell, J.R., Lienkaemper, G.W., Cromack, K. \& Cummins, K.W. (1986): Ecology of Coarse Woody Debris in Temperate Ecosystems. - Adv. Ecol. Research, 15: 133302 . 
HARMS, F.-J. (2002): Bohrung in der Grube Messel erfolgreich beendet. - Natur und Museum 132(1): 4042.

HATCHER, P.G., LeRCH, H.E. \& VeRHEYEN, T.V. (1989a): Organic geochemical studies of the transformation of gymnospermous xylem during peatification and coalification of subbituminous coal. Int. J. Coal Geol., 13: 65-97.

HATCheR, P.G., WiLson, M.A., VASsallo, A.M. \& LeRCH, H.E. (1989b): Studies of angiospermous wood in Australian brown coal by nuclear magnetic resonance and analytical pyrolysis: new insights into the early coalification process. - Int. J. Coal Geol., 13: 99-126.

HAWORTH, E.Y. (1988): Distribution of diatom taxa of the old genus Melosira (now mainly Aulacoseira) in Cumbrian waters. - in: Round, F.E. [ed.]: Algae and the Aquatic Environment. - Biopress, Bristol: 138-167.

HedGes, J.I., CoWIE, G.L. \& ERTEL, J.R. (1985): Degradation of carbohydrates and lignins in buried woods. - Geochim. Cosmochim. Acta, 49: 701-711.

HEIL, R. (1964): Kieselschwamm-Nadeln im Ölschiefer der Grube Messel bei Darmstadt. - Notizbl. hess. L.-Amt Bodenforsch., 92: 60-67.

HEIM, D. (1990): Tone und Tonminerale. - Enke, Stuttgart, 157 S.

Hein, J.R., SCHOlL, D.W., BARRon, J.A., JONES, M.G. \& Miller, J. (1978): Diagenesis of Late Cenozoic diatomaceous deposits and formation of the bottom simulating reflector in the southern Bering Sea. Sedimentology, 25: 155-181.

Hollerbach, A., Hufnagel, H. \& WeHner, H. (1977): Organisch-geochemische und -petrologische Untersuchungen an den See-Sedimenten aus der Forschungsbohrung Nördlingen 1973. - Geologica Bavarica, 75: 139-153.

HOTTENROTT, M. (1998): Mikrofloren aus den Bohrprofilen Sieblos 1994/1 und 1994/2 an der Wasserkuppe/Rhön (Eozän-Unter-Oligozän). - In: MARTINI, E. \& ROTHE, P. [Hrsg.]: Die altteriäre Fossillagerstätte Sieblos an der Wasserkuppe/Röhn. - Geol. Abh. Hessen, 104: 201-213.

HsÜ, K.J. \& KELTS, K. (1985): Swiss Lakes as a Geological Laboratory, Part I: Turbidity Currents. Naturwissenschaften, 72: 315-321.

HuC, A.Y. (1988): Aspects of depositional processes of organic matter in sedimentary basins. - In: Mattavelli, L. \& Novelli, L. [eds.]: Advances in organic geochemistry 1987. - Pergamon Press, New York, 263-272.

Huc, A.Y., Le Fournier, J., VAndenbroucke, M. \& Bessereau, G. (1990): Northern Lake Tanganyika An Example of Organic Sedimentation in an Anoxic Rift Lake. - In: KATZ, B.J. [ed.]: Lacustrine Basin Exploration - Case Studies and Modern Analogs. - AAPG Memoir, 50: 169-185.

HuCKENHOLZ, H.-G. (1983): Tertiary volcanism of the Hocheifel area. - In: FucHS, K. et al. [eds.]: Plateau uplift. Springer, Berlin usw.: 121-128.

HUCKENHOLZ, H.-G. \& BüCHEL, G. (1988): Tertiärer Vulkanismus der Hocheifel (DMG-Tagung 1988). Fortschr. Miner. 66 (Beiheft 2): 43-82.

HUSTEDT, F. (1945): Die Diatomeenflora norddeutscher Seen mit besonderer Berücksichtigung des holsteinischen Seengebietes. I-IV. - Arch. f. Hydrobiol., 41: 392-414.

HUSTEDT, F. (1957): Die Diatomeenflora des Flußsystems der Weser im Gebiet der Hansestadt Bremen. - Abh. Naturw. Ver. Bremen, 34: 181-440. 
HUTTON, A.C. (1987): Petrographic Classification of Oil Shales. - Int. J. Coal Geol., 8: 203-231.

Hutton, A.C., KantsLer, A.J., CoOK, A.C. \& MCKIRDY, D.M. (1980): Organic matter in oil shales. - APEA J., 20: 44-67.

IIJIMA, A. \& TADA, R. (1981): Silica diagenesis of Neogene diatomaceous and volcanoclastic sediments in nothern Japan. - Sedimentology, 28: 185-200.

ILER, R. (1979): The chemistry of silica. - Wiley, New York, 866 S.

INESON, J.R. (1985): Submarine glide blocks from the Lower Cretaceous of the Antarctic Peninsula. Sedimentology, 32: 659-670.

INTERNATIONALE KOMMISSION FÜR KOHLENPETROLOGIE [ICCP] (1971): Internationales Lexikon für Kohlenpetrologie. - Ergänzung zur 2. Ausgabe, CNRS, Paris, unpaginiert.

ISAACS, C.M. (1981): Porosity reduction during diagenesis of the Monterey Formation, Santa Barbara Coastal Area, California. - In: GARRISON, R.E. et al. [eds.]: The Monterey Formation and related siliceous rocks of California. - Soc. Econ. Paleontol. Mineral. Pacific Section Spec. Publ.: 257-271.

IVERSON, R.M. (1997): Physics of debris flows. - Rev. Geophys., 35: 245-296.

JACOB, H. (1989): Classification, structure, genesis and practical importance of natural solid bitumen ("migrabitumen"). - Int. J. Coal Geol., 11, 61-79.

JANKOWSKI, B: (1981): Die Geschichte der Sedimentation im Nördlinger Ries und Randecker Maar. Bochumer geol. u. geotechn. Arb., 6: 1-315.

JANKOWSKI, B. \& LITTKE, R. (1986): Das organische Material der Ölschiefer von Messel. Geowissenschaften in unserer Zeit, 4: 73-80.

JASMUND, K. (1993): Bildung und Umbildung von Tonmineralen. - In: JASMUND, K. \& LAGALY, G. [Hrsg.]: Tonminerale und Tone. - Steinkopff, Darmstadt: 168-192.

JONES, F.B. \& BOWSER, C.J. (1978): The Mineralogy and Related Chemistry of Lake Sediments. - In: LeRMAN, A. [ed.]: Lakes - Chemistry, Geology, Physics. - Springer, New York usw.: 179-235.

JøRGENSEN, B.B. (1990): The sulfur cycle of freshwater sediments: Role of thiosulfate. - Limnol. Oceanogr. 35: 1329-1342.

JYOTHI, B., SUDHAKAR, G. \& VENKATESWARLU, V. (1990): Ecological evaluation of chlorococcalean blooms - a comparative account. - J. Indian Bot. Soc., 69: 115-119.

Kadouri, A., Derenne, S., largeau, C., Casadevall, E. \& Berkaloff, C. (1988): Resistant biopolymer in the outer walls of Botryococcus braunii, B race. - Phytochemistry, 27: 551-557.

KAstneR, M., KeEnE, J.B. \& GIESKES, J.M. (1977): Diagenesis of siliceous oozes - I. Chemical controls on the rate of opal-A to opal-CT transformation - an experimental study. - Geochim. et Cosmochim.

Acta, 41: 1041-1059.

KATZ, B.J. (1990): Controls on Distribution of Lacustrine Source Rocks through Time and Space. - In: KATZ, B.J. [ed.]: Lacustrine Basin Exploration - Case Studies and Modern Analogs. - AAPG Memoir, 50: 61-76.

KEENE, J.B. (1976): The distribution, mineralogy, and petrography of biogenic and authigenic silica from the Pacific Basin. - Ph.D. thesis, Univ. Calif. San Diego, Scripps Inst. Ocean., 264 S. [zitiert in FÜCHTBAUER, H. (1988a)] 
KELTS, K. (1988): Environments of deposition of lacustrine petroleum source rocks: an introduction. - In: FLEET, A.J. et al. [eds.]: Lacustrine Petroleum Source Rocks. - Geol. Soc. Spec. Publ., 40: 3-26.

KJENSMO, J. (1968): Iron as the primary factor rendering lakes meromictic, and related problems. - Mitt. int. Verein. Limnol., 14: 83-93.

Kölschbach, K.-H., MeYer, W., SteTS, J. \& Thon, B. (1993): Das Manderscheider Antiklinorium in der Südwest-Eifel (Rheinisches Schiefergebirge). - Decheniana 146: 296-314.

KomÁREK J. \& FotT, B. (1983): Das Phytoplankton des Süßwassers. - In: Die Binnengewässer, Bd. 16, 7. Teil, 1. Hälfte, Schweizerbart, Stuttgart, 1044 S.

KomÁREK, J \& MARVAN, P (1992): Morphological Differences in Natural Populations of the Genus Botryococcus (Clorophyceae). - Arch. Protistenkd., 141: 65-100.

KöSTER, H.M. (1974): Ein Beitrag zur Geochemie und Entstehung der Oberpfälzischen Kaolin-FeldspatLagerstätten. - Geol. Rdsch., 63: 655-689.

KOVACH, W.L., BATTEN, D.J. (1994): Association of palynomorphs and palynodebris with depositional environments: quantitative approaches. - In: TRAVERSE, A. [ed.]: Sedimentation of organic particles. Cambridge Univ. Press, Cambridge: 391-407.

KoVÁČIK, L. (1975): Taxonomic review of the genus Tetraedron (Chlorococcales). - Arch. Hydrobiol., Suppl. 46: 354-391.

KRAmmer, K. \& LANGe-BeRtalot, H. (1991): Bacillariophyceae, 3. Teil: Centrales, Fragilariaceae, Eunotiaceae. - in: PASCHER, A. [Begr.]: Süßwasserflora von Mitteleuropa, 2(3). - G. Fischer, Stuttgart, 576 S.

KRIBEK, B. (1975): The Origin of Framboidal Pyrite as a Surface Effect of Sulphur Grains. - Mineral. Deposita, 10: 389-396.

KRINSLEY, D.H., PYE, K., Boggs, S \& ToVEY, N.K. (1998): Backscattered Scanning Electron Microscopy and Image Analysis of Sediments and Sedimentary Rocks. - Cambridge Univ. Press, Cambridge, $193 \mathrm{~S}$

LAMBERT, A.M. \& LÜTHI, S.M. (1977): Lake circulation induced by density currents: an experimental approach. - Sedimentology, 24: 735-741.

Largeau, C., Casadevall, E., Kadouri, A. \& Metzger, P. (1984): Formation of Botryococcus-derived kerogens - Comparative study of immature torbanites and of the extant alga Botryococcus braunii. Org. Geochem. 6: 327-332.

Largeau, C., Derenne, S., Casadevall, E., Kadouri, A. \& Sellier, N. (1986): Pyrolysis of immature torbanite and of the resistant biopolymer (PRB A) isolated from extant alga Botryococcus braunii. Mechanism of formation and structure of torbanite. - Org. Geochem., 10: 1023-1032.

LEEDER, M.R. (1982): Sedimentology: Process and Product. - Allen \& Unwin, London, 344 S.

LENZ, O. K. (2000): Paläoökologie eines Küstenmoores aus dem Eozän Mitteleuropas am Beispiel der Wulfersdorfer Flöze und deren Begleitschichten (Helmstedter Oberflözgruppe, Tagebau Helmstedt). http://webdoc.sub.gwdg.de/diss/2000/lenz/index.htm, 230 S.

LEPSIUS, R. (1887-1892): Geologie von Deutschland und den angrenzenden Gebieten. Erster Teil: Das westliche und südliche Deutschland. - J. Engelhorn, Stuttgart, $800 \mathrm{~S}$. 
LEWIN, J.C. (1961): The dissolution of silica from diatom walls. - Geochim. et Cosmochim. Acta, 21: 182198.

LIEBIG, K. (1998): Fossil Microorganisms from the Eocene Messel Oil Shale of Southern Hesse, Germany. - Kaupia, 7: 1-95.

LIEBIG, V. (2001): Neuaufnahme der Forschungsbohrungen KB 1,2,4,5 und 7 von 1980 aus der Grube Messel (Sprendlinger Horst, Südhessen). - Kaupia, 11: 3-68.

LITTKE, R. (1993): Deposition, Diagenesis and Weathering of Organic Matter-Rich Sediments. - Lekture Notes in Earth Sciences, 47: 1-216.

LIU, S.Y., TAYLOR, G.H. \& SHIBAOKA, M. (1982): Biochemical gelification and the nature of some huminite macerals. - in: MALLETT, C.W. [ed.]: Coal resources - origin, exploration and utilization in Australia. Austr. Coal Geol., 4: 145-152.

LÖHNERTZ, W. (1978a): Geologie und Heimatkunde - Tonabbau und Ziegelfabrikation im Liesertal bei Manderscheid - Jb. Kreis Bernkastel-Wittlich, 1978: 220-227.

LÖHNERTZ, W. (1978b): Zur Altersstellung der tiefliegenden fluviatilen Tertiärablagerungen der SE-Eifel (Rheinisches Schiefergebirge). - N. Jb. Geol. Paläont. Abh., 156: 179-206.

LÖHNERTZ, W. (1982): Forschungsbohrung Eckfeld. - Jb. Kreis Bernkastel-Wittlich, 1982: 266-267.

LÖHNERTZ, W. (1994): Grundzüge der morphologischen Entwicklung der südlichen Eifel im ältesten Tertiär - In: NEUFFER, F.O. et al. [Hrsg.]: Fossillagerstätte Eckfelder Maar. - Mainzer naturwiss. Archiv, Beiheft 16: 17-38.

LOHMAN, K.E. (1960): The ubiquitous diatom - A brief survey of the present state of knowledge. - Am. J. Sci., 258-A: 180-191.

LORENZ, V. (1987): Phreatomagmatism and its relevance. - Chem. Geol., 62: 149-156.

LORENZ, V. (2000): Formation of maar-diatreme volcanoes. - Terra Nostra 2000/6: International Maar Conference, Daun/Vulkaneifel: 284-291.

LOVE, L.G. (1957): Micro-organisms and the presence of syngenetic pyrite. - Geol. Soc. London Quart. J., 113: $429-440$.

LOVE, L.G. (1962a): Biogenic primary sulfide of the Permian Kupferschiefer and marl slate. - Econ. Geol., 57: 350-366.

LOVE, L.G. (1962b): Further studies on micro-organisms and the presence of syngenetic pyrite. Palaeontology, 5: 444-459.

LOVE, L.G. \& ZIMMERMAN, D.O. (1961): Bedded pyrite and micro-organisms from the Mount Isa shale. Econ. Geol., 56: 873-896.

LOWE, D.R. (1975): Water escape structures in coarse-grained sediments. - Sedimentology, 22: $157-204$.

LOWE, D.R. (1979): Sediment gravity flows: their classification and some problems of application to natural flows and deposits. - Soc. Econ. Paleontol. Mineral. Spec. Publ., 27: 75-82.

LOWE, D.R. (1982): Sediment gravity flows: II. Depositional models with special reference to the deposits of high-density turbidity currents. - J. Sed. Petrol., 52: 279-297.

LOWE, D.R. (1988): Suspended-load fallout rate as an independent variable in the analysis of current structures. - Sedimentology, 35: 765- 776. 
LUDLAM, S.D. (1974): Fayetteville Green Lake, New York. 6. The role of turbidity currents in lake sedimentation. - Limnol. Oceanogr., 19: 656-664.

LUTZ, H. (1988): Die Arthropoden-Thanatozönose vom "Eckfelder Maar" - Ein erster Überblick. - Mainzer naturwiss. Archiv, 26: 151-155.

LUTZ, H. (1991): Fossilfundstelle Eckfelder Maar - Beiträge zur Flora und Fauna des Mitteleozäns der Eifel. - Landessamml. f. Naturkunde Rheinl.-Pfalz, Mainz, 51 S.

LUTZ, H. (1993a): Zur Sedimentologie der Leithorizonte des "Eckfelder Maares" bei Manderscheid/Eifel (Mittel-Eozän; Deutschland). - Mainzer naturwiss. Archiv, 31: 65-83.

LUTZ, H. (1993b): Zur Taphonomie der aquatischen und terrestrischen Fauna des "Eckfelder Maares" (Mittel-Eozän; Deutschland): Ergebnisse aus den Grabungen 1990-1992. - Mainzer naturwiss. Archiv, 31: 85-113.

LUTZ, H. (1993c): Eckfeldapis electrapoides nov.gen.n.sp., eine "Honigbiene" aus dem Mittel-Eozän des "Eckfelder Maares" bei Manderscheid/Eifel, Deutschland (Hymenoptera: Apidae, Apinae). - Mainzer naturwiss. Archiv, 31: 177-199.

LUTZ, H. (1993d): The Middle-Eocene "Fossillagerstätte Eckfelder Maar" (Eifel, Germany). - Kaupia, 2: 21-25.

LUTZ, H. (1993e): Grabungskampagne im "Eckfelder Maar": 1992. - Mitt. Rhein. Naturforsch. Ges., 14: 53-59.

LUTZ, H. (1998): Zur Korrelation von Fazies und Fossilführung im Eckfelder Maar (Mittel-Eozän, Vulkaneifel, Deutschland). - Mainzer naturwiss. Archiv, 36: 39-46.

LUTZ, H. (1999): Forschungsprojekt Eckfelder Maar: Grabungskampagne 1998. - Mitt. Rhein. Naturforsch. Ges., 20: 28-31.

LUTZ, H. (2000): Correlation of facies and fossil contents in meromictic maar lakes - The example Eckfeld Maar. - Terra Nostra 2000/6: International Maar Conference, Daun/Vulkaneifel: 301-308.

LUTZ, H. \& NeUfFeR, F.O. (1997): Eckfeld Maar. - Terra Nostra 97/8: $7^{\text {th }}$ International Symposium on Paleolimnology, Heiligkreuztal, Excursion Guide: B55-B60.

LUTZ, H. \& Neuffer, F. O. (2001): Ein Klimaarchiv mit Haut und Haaren. - Forschung, 1/2001: 12-15.

LUTZ, H., FRANKENHÄUSER, H. \& NeUfFer, F.O. (1998): Fossilfundstätte Eckfelder Maar - Archiv eines mitteleozänen Lebensraums in der Eifel. - Landessamml. f. Naturkunde Rheinl.-Pfalz, Mainz, $51 \mathrm{~S}$.

Lutz, H., Neuffer, F.O., Harms, F.-J., SchaAl, S., Micklich, N., Gruber, G., Schweigert, G. \& Lorenz, V. (2000): Tertiary maars as fossil deposits: Eckfeld, Messel, Randeck, Höwenegg, Öhningen. - In: NEUfFER, F.O. \& LUTZ, H [eds.]: Field Trip Guidebook, International Maar Conference Daun/Vulkaneifel, 2000. - Mainzer naturwiss. Archiv, Beiheft 24: 125-160.

MAI, D.H. (1995): Tertiäre Vegetationsgeschichte Europas. - Fischer, Jena usw., 691 S.

MARR, J.G., HARFF, P.A., ShANmUgam, G. \& PARKER, G. (2001): Experiments on subaqueous sandy gravity flows: The role of clay and water content in flow dynamics and depositional structures. - Geol. Soc. Am. Bull., 113: 1377-1386.

MARTINI, E. \& RIETSCHEL, S. (1978): Lösungserscheinungen an Schwammnadeln im Messeler Ölschiefer (Mittel-Eozän). - Erdöl-Erdgas-Z., 94: 94-97. 
MARTINI, E. \& ROTHE, P. (1988): Die unteroligozäne "Braunkohlen"-Lagerstätte Sieblos an der Wasserkuppe/Rhön (Geologie, Paläontologie, Paläoökologie, Paläogeographie). - Beitr. Naturkde. Osthessen, 24: 7-25.

MARTINI, E. \& Rothe, P. (1993): Sieblos - A Lower Oligocene Messel-Type Deposit. - Kaupia, 2: 39-53.

MARTINI, E. \& SCHILLER, W. (1995): Amphidisken der Schwammgattung Ephydatia im Unter-Oligozän von Sieblos/Rhön. - Beitr. Naturkde. Osthessen, 31: 17-21.

MARTINI, E. \& Schrader, H. (1988): Diatomeen, Chrysophyceen, Nannoplankton, Dinoflagellaten und Schwammnadeln aus dem Unter-Oligozän von Sieblos/Rhön. - Beitr. Naturkde. Osthessen, 24: 5560.

Mertz, D.F., SWisher, C.C., Franzen, J.L., Neuffer, F.O. \& LUTZ, H. (2000): Numerical dating of the Eckfeld maar fossil site, Eifel, Germany: a calibration mark for the Eocene time scale. Naturwissenschaften, 87: 270-274.

MEYER, W. (1994): Geologie der Eifel. - Schweitzerbart, Stuttgart, 618 S.

MeYer, W., PIRRUng, B. M. \& StetS, J. (1994): Der variscische Sockel in der Umgebung des Eckfelder Maares. - In: NEUFFER, F.O. et al. [Hrsg.]: Fossillagerstätte Eckfelder Maar. - Mainzer naturwiss. Archiv, Beiheft 16: 9-16.

MiCKLICH, N. \& WUTTKE, M. (1988): Ein Percoidei-Fund mit "Weichteilerhaltung" aus dem Eozän von Eckfeld bei Manderscheid (SW-Eifel, Bundesrepublik Deutschland). - Mainzer naturwiss. Archiv, 26: 75-106.

MidDleton, G.V. (1993): Sediment deposition from turbidity currents. - Annu. Rev. Earth Planet. Sci., 21: 89-114.

MINGRAM, J. (1994): Sedimentologie und Zyklizität laminierter eozäner Ölschiefer von Eckfeld/Eifel. - In: NeUfFER, F.O. et al. [Hrsg.]: Fossillagerstätte Eckfelder Maar. - Mainzer naturwiss. Archiv, Beiheft 16: 55-86.

MINGRAM, J. (1997): Eckfeld Dry Maar Sediments. - Terra Nostra 97/8: $7^{\text {th }}$ International Symposium on Paleolimnology, Excursion Guide: B60-B65.

MINGRAM, J. (1998): Laminated Eocene maar-lake sediments from Eckfeld (Eifel region, Germany) and their short-term periodicities. - Palaeogeogr., Palaeoclimat., Palaeoecol., 140: 289-305.

MORI, S.,SAIJO, Y. \& MiZUNO, T. (1984): Limnology of Japanese lakes and ponds. - In: TAUB, F. [ed.]: Lakes and Reservoirs. Ecosystem of the World. - Elsevier, Amsterdam: 303-330.

MÖRS, T. (1995): Die Sedimentationsgeschichte der Fossillagerstätte Rott und ihre Alterseinstufung anhand neuer Säugetierfunde (Oberoligozän, Rheinland). - Cour. Forsch.-Inst. Senckenberg, 187: 1101.

MüLLER, W.E.G., ZAHN, R.K. \& MAIDHOF, A. (1982): Spongilla gutenbergiana n. sp., ein Süßwasserschwamm aus dem Mittel-Eozän von Messel. - Senckenbergiana lethaea, 63: 465-472.

Müller-Sohnius, D., HORN, P. \& HuCKenHOLZ, H. G. (1989): Kalium-Argon-Datierungen an tertiären Vulkaniten der Hocheifel (BRD) - Chem. Erde, 49: 119-136.

MüLLER-WILMES, B. (1989): Erste diatomeenkundliche Untersuchungen im "Eckfelder Maar". - Bericht für das Naturhistorische Museum Mainz / Landessammlung f. Naturkunde Rheinl.-Pfalz, unveröff., 4 S. [zitiert in WILDE, V., FRANKENHÄUSER, H. \& LUTZ, H. (1993)]. 
MÜNTER, C. (1999): Kohlepetrographische Untersuchungen an den Proben des oligozänen Ölschiefers von Enspel/Westerwald. - unveröff. Dipl.-Arb., Georg-August-Univ. Göttingen, 96 S.

MULDER, T. \& ALEXANDER, J. (2001): The physical character of subaqueous sedimentary density flows and their deposits. - Sedimentology, 48: 269-299.

MURATA, K.J., FRIEDMAN, I., GLEASON, J.D. (1977): Oxygen isotope relations between diagenetic silica minerals in Monterey Shale, Temblor Range, California. - Am. J. Sci, 277: 259-272.

NARDIN, T.R., HEIN, F.J., GoRSLINE, D.S. \& EDWARDS, B.D. (1979): A review of mass movement processes, sediment and acoustic characteristics, and contrasts in slope and base-of-slope systems versus canyon-fan-basin floor systems. - In: DOYLE, L.J. \& PILKEY, O.H. [Eds.]: Geology of Continental Slopes. - SEPM Spec. Publ., 27: 61-73.

NEGENDANK, J.F.W., IRON, G. \& LINDEN, J. (1982): Ein eozänes Maar bei Eckfeld nordöstlich Manderscheid (SW-Eifel). - Mainzer Geowiss. Mitt., 11: 157-172.

NeLSON, C.H. (1967): Sediments of Crater Lake, Oregon. - Bull. Geol. Soc. Am., 78: 833-848.

NeuBERT, E. (1995): Die Gastropoda des "Eckfelder Maares" (Mittel-Eozän). - Mainzer naturwiss. Archiv, 33: 53-61.

NeuHAus, A. (1940): Über die Erzführung des Kupfermergels der Haaseler und der Gröditzer Mulde in Schlesien. - Zeitschr. f. angew. Mineral., 2: 304-343.

NICKEL, B. (1994): Neue palynologische Untersuchungen am mitteleozänen Ölschiefer von Eckfeld bei Manderscheid/Eifel. - Erste Ergebnisse. - Mainzer naturwiss. Archiv, 32: 7-25.

NiCKEL, B. (1996): Die mitteleozäne Mikroflora von Eckfeld bei Manderscheid/Eifel. - Mainzer naturwiss. Archiv, Beiheft 18: 1-146.

NYGAARD, G. (1956): Ancient and Recent Flora of Diatoms and Chrysophyceae in Lake Gribs $\emptyset$. - Folia Limnol. Scandinavica, 8: 32-94.

OEHLER, J.H. (1975): Origin and distribution of silica lepispheres in porcelanite from the Monterey Formation of California. - J. Sed. Petrol., 45: 252-257.

OLSEN, P.E. (1990): Tectonic, Climatic, and Biotic Modulation of Lacustrine Ecosystems - Examples from Newark Supergroup of Eastern North America. - In: KATZ, B.J. [ed.]: Lacustrine Basin Exploration Case Studies and Modern Analogs. - AAPG Memoir, 50: 209-224.

PALMER, B.A. \& SHAWKEY, E.P. (2001): Lacustrine-fluvial transitions in a small intermontane valley, Eocene Challis volcanic field, Idaho. - In: WHITE, J.D.L. \& RIGGS, N.R. [eds.]: Volcaniclastic Sedimentation in Lacustrine Settings. - Spec. Publs. int. Assoc. Sediment., 30: 179-198.

PANKOW, H. (1976): Algenflora der Ostsee, II. Plankton. - Fischer, Stuttgart, 493 S.

PARKER, J.I. \& EDGINGTON, D.N. (1976): Concentration of diatom frustules in Lake Michigan sediment cores. - Limnol. Oceanogr., 21: 887-893.

Pedersen, G.K., Larsen, L.M., Pedersen, A.K. \& HJortKJ/er, B.F. (1998): The syn-volcanic Naajaat lake, Paleocene of West Greenland. - Palaoegeogr., Palaeoclimat., Palaeoecol., 140: 271-287.

PfLUG, H.D. (1959): Die Deformationsbilder im Tertiär des rheinisch-saxonischen Feldes. - Freib. Forsch.-H., C71: 1-110.

PHILLIPS, W.R. \& GRIFFEN, D.T. (1981): Optical Mineralogy. - Freemann \& Co., San Francisco, 677 S. 
Pielou, E.C. (1984): The Interpretation of Ecological Data. - Wiley \& Sons, New York, 263 S.

PIPER, D.J.W. \& StoW, D.A.V. (1991): Fine-Grained Turbidites. - In: EINSELE, G. et al. [eds.]: Cycles and Events in Statigraphy. - Springer, Berlin usw.: 360-376.

PIRRUNG, B.M. (1992a): Zur Frage der Entstehung eozäner Sedimente im "Eckfelder Maar" bei Manderscheid, Südwesteifel. - Mitt. Pollichia, 79: 139-158.

PIRRUNG, B.M. (1992b): Geologische und geophysikalische Untersuchungen am tertiären "Eckfelder Maar", Südwesteifel. - Mainzer naturwiss. Archiv, 30: 3-21.

PIRRUNG, B.M. (1993): Weitere Sondierungen und geophysikalische Untersuchungen am eozänen Eckfelder Maar bei Manderscheid, Südwesteifel. - Mainzer naturwiss. Archiv, 31: 37-63.

PIRRUNG, B.M. (1998): Zur Entstehung isolierter alttertiärer Seesedimente in Zentraleuropäischen Vulkanfeldern. - Mainzer naturwiss. Archiv, Beiheft 20: 1-117.

PIRRUNG, M., BÜChEL, G. \& JACOBY, W. (2001): The Tertiary volcanic basin of Eckfeld, Enspel and Messel (Germany). - Z. dt. geol. Ges., 152/1: 27-59.

PolLINGHER, U. (1990): Effects of Latitude on Phytoplankton Composition and Abundance in Large Lakes. - In: TILZER, M.M. \& SERRUYA, C. [eds.]: Large Lakes. - Springer, Berlin usw.: 368-402.

PostMA, D. (1982): Pyrite and siderite formation in brackish and freshwater swamp sediments. - Am. J. Sci., 282: 1151-1183.

PostmA, G. (1986): Classification for sediment gravity-flow deposits based on flow conditions during sedimentation. - Geology, 14: 291-294.

PRESTON, R.D. (1974): The Physical Biology of Plant Cell Walls. - Chapman \& Hall, London, 491 S.

RAYNER, A.D.M. \& BODDY, L. (1988): Fungal Decomposition of Wood - Its Biology and Ecology. - Wiley \& Sons, Chichester, $587 \mathrm{~S}$.

RICHTER, G. \& WUTTKE, M. (1995): Der Messeler Süßwasser-Kieselschwamm Spongilla gutenbergiana, eine Ephydatia. - Natur und Museum, 125(4): 134-135.

RICHTER, G. \& WUTTKE, M. (1999): Lutetiospongilla heili n. gen. n. sp. und die eozäne Spongillidenfauna von Messel. - Cour. Forsch.-Inst. Senckenberg, 216: 183-195.

Riegel, W., BlickWede, H., BuLlWinkEL, V., LenZ, O. \& WiLDE, V. (2002): Eine Blätterkohle aus dem Mitteleozän von Helmstedt (Tagebau Helmstedt, Bezirk Braunschweig). - Cour. Forsch.-Inst. Senckenberg, im Druck.

Riegel, W., Bode, T., Hammer, J., Hammer-Schiemann, G., Lenz, O. \& Wilde, V. (1999): The palaeoecology of the Lower and Middle Eocene at Helmstedt, northern Germany - A study in contrast. - Acta Palaeobot. Suppl. 2: 349-358.

RIGGs, N.R., ORT, M.H., White, J.D.L., WILSON, C.J.N, HOUghton, B.F. \& CLARKSON, R. (2001): Post-1.8ka marginal sedimentation in Lake Taupo, New Zealand: effects of wave energy and sediment supply in a rapidly rising lake. - In: WHITE, J.D.L. \& RIGGS, N.R. [eds.]: Volcaniclastic Sedimentation in Lacustrine Settings. - Spec. Publs. int. Assoc. Sediment., 30: 151-177.

Round, F.E. (1981): The ecology of algae. - Cambridge Univ. Press, Cambridge, 653 S.

Round, F.E. \& BROOK, A.J. (1959): The phytoplankton of some Irish loughs and an assessment of their trophic status. - Proc. Roy. Ir. Acad., 60: 168-191. 
Round, F.E., CRAWFORD, R.M. \& MANN, D.G. (1990): The Diatoms. - Cambridge Univ. Press, Cambridge: $747 \mathrm{~S}$.

RUSSELL, N.J. \& BARRON, P.F. (1984): Gelification of Victorian Tertiary soft brown coal wood. II. Changes in chemical structure associated with variation in the degree of gelification. - Int. J. Coal Geol., 4: 119142.

RUST, J. (2002): Diversität und Entwicklung der Insekten im europäischen Alttertiär. - GEO 2002, Programm und Kurzfassungen. - Schriftr. Dtsch. Geol. Ges., 21: 287.

RUTTNER, F. (1952): Planktonstudien der Deutschen Limnologischen Sunda-Expedition. - Arch. Hydrobiol., Suppl. 21 (1/2): 1-274.

RYBAK, M., RYBAK, I. \& NICHOLLS, K. (1991): Sedimentary chrysophycean cyst assemblages as paleoindicators in acid sensitive lakes. - J. Paleolimnol., 5: 19-72.

SACHSE, M. \& LUTZ, H. (2001): Forschungsprojekt Eckfelder Maar: Jahresbericht 2000. - Mitt. Rhein. Naturforsch. Ges., 22: 36-41.

SANDGREN, C.D. (1988): The ecology of chrysophyte flagellates: their growth and perennation strategies as freshwater phytoplankton. - in: SANDGREN, C.D. [ed.]: Growth and Reproductive Strategies of Freshwater Phytoplankton. - Cambridge Univ. Press, Cambridge: 9-104.

SANDGREN, C.D. (1991): Chrysophyte reproduction and resting cysts: a paleolimnologist's primer. - J. Paleolimnol., 5: 1-9.

SATAKE, K. (1977): Microbial sulphate reduction in a volcanic acid lake having pH 1.8 to 2.0. - Jap. J. Limnol., 38: 33-35. [zitiert in MORI, S., SAIJO, Y. \& MIZUNO, T. (1984)]

SCHILLER, W. (1998): Kieselige Mikrofossilien aus dem Unter-Oligozän von Sieblos/Rhön. - Geol. Abh. Hessen, 104: 173-199.

SCHILLER, W. (1999): Kieselige Thekamöben aus dem Mittel-Eozän des Eckfelder Maares in der Eifel. Mainzer naturwiss. Archiv, 37: 55-62.

SCHILLER, W. (2000): Feinstratigraphische Untersuchungen der Kernbohrung Enspel 1991(2) unter Berücksichtigung der kieseligen Mikrofossilien. - Mainzer naturwiss. Archiv, 38: 39-91.

SCHILLER, W. \& LANGE-BERTALOT, H. (1997): Eolimna martinii n. gen., n. sp. (Bacillariophyceae) aus dem Unter-Oligozän von Sieblos/Rhön im Vergleich mit ähnlichen rezenten Taxa. - Paläont. Zeitschr., 71 (3/4): 163-172.

SCHNEIDER, J., RÖHRS, J. \& JÄGER, P. (1990): Sedimentation and Eutrophication History of Austrian Alpine Lakes. - In: TILZER, M.M. \& SERRUYA, C. [eds.]: Large Lakes. - Springer, Berlin usw.: 316-335.

SCHNEIDERHÖHN, H. (1923): Chalkographische Untersuchungen des Mansfelder Kupferschiefers. - N. Jb. Mineral., Geol. und Paläont., B 47: 1-38.

Schwertmann, U. \& Niederbudde, E.-A. (1993): Tonminerale in Böden. - In: JAsmund, K. \& Lagaly, G. [Hrsg.]: Tonminerale und Tone. - Steinkopff, Darmstadt: 212-265.

ScotT, A.C. (2002): Coal petrology and the origin of coal macerals: a way ahead? - Int. J. Coal Geol., 50: 119-134.

SeLLEY, R.C. (1985): Elements of Petroleum Geology. - Freeman \& Co., New York, 449 S. 
ShANKS, W.C., SeYfried, W.E., MeYER, W.C. \& O'NeIL, T.J. (1976): Mineralogy of Oil Shale. - In: Yen, T.F. \& CHILINGARIAN, G.V. [eds.]: Developments in Petroleum Science, 5, Elsevier, Amsterdam: 81102.

SHAW, G. (1971): The chemistry of sporopollenin. - In: BROOKS, J. et al. [eds.]: Sporopollenin. Academic Press, London: 305-350.

SHERWOOD, N.R. (1984): Final Project Report to the Department of National Development and Energy on NERDDC Program Project 79/9014, Low Rank Oil Shales: Part I - Organic Petrology. - Univ. Wollongong, Australia, $293 \mathrm{~S}$.

SIMONSEN, R. (1979): The diatom system: ideas on phylogeny. - Bacillaria, 2: 9-71.

SMITH, G.A. (1986): Coarse-grained nonmarine volcaniclastic sediment: terminology and depositional process. - Geol. Soc. Am. Bull., 97: 1-10.

SMOL, J.P. (1988): Chrysophycean microfossils in paleolimnological studies. - Palaeogeogr., Palaeoclimat., Palaeoecol., 62: 287-297.

STARMACH, K. (1985): Chrsophyceae und Haptophyceae. - in: PASCHER, A. [Begr.]: Süßwasserflora von Mitteleuropa, 1. - G. Fischer, Stuttgart, $515 \mathrm{~S}$.

STEIN, C.L. \& KIRKPATRICK, R.J. (1976): Experimental porcelanite recristallization kinetics: A nucleation and growth model. - J. Sed. Petrol., 46: 430-435.

STOERMER, E.F. \& LADEWSKI, T.B. (1976): Apparent optimal temperatures for the occurrence of some common phytoplankton species in southern Lake Michigan. - Great Lakes Res. Div., 18, 49 S.

STOUT, J.D., GOH, K.M. \& RAFTER, T.A. (1981): Chemistry and turnover of naturally occuring resistant organic compounds in soil. - Soil Biochem., 5: 1-73.

STOUT, S.A. \& SPACKMAN, W. (1987): A Microscopic Investigation of Woody Tissues in Peats: Some Processes Active in the Peatification of Ligno-Cellulosic Cell Walls. - Int. J. Coal Geol., 8: 55-68.

Stout, S.A., Spackman, W., Boon, J.J., KISTEMAKer, P.G. \& Bensley, D.F. (1989): Correlations between the microscopic and chemical changes in wood during peatification and early coalification: a canonical variant study. - Int. J. Coal Geol., 13: 41-64.

STURM, M. (1979): Origin and composition of clastic varves. - In: SCHLÜCHTER, C. [ed.]: Moraines and Varves. - Proc. INQUA symp. on genesis and lithology of Quaternary deposits, Zurich 1978: 281-285.

STURM, M. \& MATTER, A. (1978): Turbidites and varves in Lake Brienz (Switzerland): deposition of clastic detritus by density currents. - Spec. Publs. int. Assoc. Sediment., 2: 147-168.

SWEENEY, R.E. \& KAPLAN, I.R. (1973): Pyrite framboid formation: laboratory synthesis and marine sediments. --Econ. Geol., 68: 618-634.

TADA, R. (1991): Compaction and Cementation in Siliceous Rocks and Their Possible Effect on Bedding Enhancement. - In: EINSELE, G. et al. [eds.]: Cycles and Events in Statigraphy. - Springer, Berlin usw.: 480-491.

TADA, R. \& IIJIMA, A. (1983): Petrology and diagenetic changes of Neogene siliceous rocks in northern Japan. - J. Sed. Petrol., 53: 911-930.

TALBOt, M.R. \& AlLEN, P.A. (1996): Lakes. - In: READING, H.G. [ed.]: Sedimentary Environments: Process, Facies and Stratigraphy. - Blackwell, Oxford: 83-124. 
TAPPAN, H. (1980): The paleobiology of plant protists. - W.H. Freeman, San Francisco, 1028 S.

TAULBEE, D.N., SeiBert, E.D., BARRON, L.S. \& RobL, T.L. (1990): Comparison of maceral group chemistries for a New Albany and an Oil Shale kerogen. - Energy and Fuels, 4: 254-263.

TAYloR, G.H., TeICHMÜller, M., DAvis, A., Diessel, C.F.K., LitTKe, R. \& RobeRT, P. (1998): Organic Petrology. - Geb. Borntraeger, Berlin, Stuttgart, 704S.

TEICHMÜLLER, M. (1974): Über neue Macerale der Liptinit-Gruppe und die Entstehung von Micrinit. Fortschr. Geol. Rheinld. u. Westf., 24: 37-64.

TEICHMÜLLER, M. (1989): The genesis of coal from the viewpoint of coal petrology. - Int. J. Coal Geol., 12: $1-87$.

TEICHMÜLLER, M. \& TEICHMÜLLER, R. (1982): The geological basis of coal formation. - In: STACH, E. et al. [eds.]: Stach's Textbook of Coal Petrology. - Gebr. Borntraeger, Berlin, 5-86.

TEMPERLEY, B.N. (1936): The boghead controversy and the morphology of the boghead algae. Transactions of the Royal Society of Edinburgh, 58: 855-868.

TILZER, M.M. (1990): Environmental and Physiological Control of Phytoplankton Productivity in Large Lakes. - In: TILZER, M.M. \& SERRUYA, C. [eds.]: Large Lakes. - Springer, Berlin usw.: 339-367.

TISSOT, B.P. \& WELTE, D.H. (1984): Petroleum Formation and Occurence. - Springer, Berlin usw., 699 S.

TRAVERSE, A. (1955): Occurrence of the oil-forming alga Botryococcus in lignites and other Tertiary sediments. - Micropaleontology, 1: 343-350.

TRAVERSE, A. (1992): Organic fluvial sediment: palynomorphs and 'palynodebris' in the lower Trinity River, Texas. - Annals of the Missouri Botanical Garden, 79: 110-125.

TRÖGER, W.E. (1967): Optische Bestimmung der gesteinsbildenden Minerale, Teil 2 Textband. Schweizerbart, Stuttgart, $822 \mathrm{~S}$.

TRUDINGER, P.A. (1992): Bacterial sulfate reduction: current status and possible origin. - In: SCHIDLOWSKI, M. et al. [eds.]: Early Organic Evolution. - Springer, Berlin: 367-377.

TUCKER, M.E. (2001): Sedimentary Petrology: An Introduction to the Origin of Sedimentary Rocks. Blackwell, Oxford, $262 \mathrm{~S}$.

TYSON, R.V. (1995): Sedimentary Organic Matter. - Chapman \& Hall, London, 615 S.

Vincent, W.F., Wurstbaug, W., Neale, P.J. \& Richerson, P.J. (1986): Polymixis and algae production in a tropical lake: latitudinal effects on the seasonality of photosynthesis. - Freshwater Biol., 16: 781803.

VolKMER-RIBEIRO, C. (1990): A new insight into the systematics, evolution and taxonomy of freshwater sponges. - In: RüTZLER, K. [ed.]: New Perspectives in Sponge Biology - Smithsonian Inst. Press, Washington, D.C.: 323-331.

Volkmer-RibeiRo, C. \& Reitner, J. (1991): Renewed Study of the Type Material of Palaeospongilla chubutensis Ott and Volkheimer (1972). - In: REITNER, J. \& KEUPP, H. [eds.]: Fossil and Recent Sponges. - Springer, Berlin usw.: 121-133.

VON DECHEN, H. (1884): Geologische und Paläontologische Übersicht der Rheinprovinz und der Provinz Westfalen, sowie einiger angrenzenden Gegenden. - A. Henry, Bonn, 933 S. 
VON DER BRELIE, G. \& WOLF, M. (1981): Zur Petrographie und Palynologie heller und dunkler Schichten im rheinischen Hauptbraunkohlenflöz. - Fortschr. Geol. Rheinld. u. Westf., 29: 95-163.

VON DER BRELIE, G. (1977): Die Pollenflora der See-Sedimente in der Forschungsbohrung Nördlingen 1973. - Geologica Bavarica, 75: 111-125.

VON DER BRElie, G., QuitzoW, H.W. \& StAdLER, G. (1969): Neue Untersuchungen im Alttertiär von Eckfeld bei Manderscheid (Eifel). - Fortschr. Geol. Rheinld. u. Westf., 17: 27-40.

VON RAD, U. (1979): $\mathrm{SiO}_{2}$-Diagenese in Tiefseesedimenten. - Geol. Rundsch., 68: 1025-1036.

Vos, H. \& MingRAM, J. (2002): Solare Fingerabdrücke in eozänen Laminiten aus dem Eckfelder Maar. GEO 2002, Programm und Kurzfassungen. - Schriftr. Dtsch. Geol. Ges., 21: 342.

WAKE, L.V. \& HILLEN, L.W. (1980): Study of a "bloom" of the oil-rich alga Botryococcus braunii in the Darwin River Reservoir. - Biotechnology and Bioengineering, 22: 1637-1656.

WARD, J.H. (1963): Hierachical grouping to optimize an objective function. - J. Am. statist. Assoc., 58 : 236-244.

WeBER, C.O. (1853): Ueber das Braunkohlenlager von Eckfeld in der Eifel. - Verh. naturhist. Vereins preuss. Rheinl. u. Westph., 10: 409-415.

Weber, J. \& ZIMMERLE, W. (1985): Pyroclastic detritus in the lacustrine sediments of the Messel Formation. - Senckenb. letheae, 66: 171-176.

Wehner, H., Hufnagel, H., Teschner, M. \& Koester, J. (1992): Maturity- and Facies-Controlled Composition of the Organic Matter of Selected Oil Shales. - In: WHELAN, J.K. \& FARRINGTON, J.W. [eds.]: Organic Matter: Productivity, Accumulation, and Preservation in Recent and Ancient Sediments. - Columbia Univ. Press, New York, 453-468.

WeISSENFELS, N. (1989): Biologie und Mikroskopische Anatomie der Süßwasserschwämme (Spongillidae). - Fischer, Stuttgart und New York, $110 \mathrm{~S}$.

WeRFF, A. VAN DER \& HULS, H. (1957-1974): Diatom Flora of the Netherlands. - Koeltz, Königstein: keine Seitenangabe.

WESTRICH, J.T. \& BERNER, R.A. (1984): The role of sedimentary organic matter in bacterial sulfate reduction: The G model tested. - Limnol. Oceanogr., 29: 236-247.

Wetzel, R.G. (1983): Limnology. - Saunders College Publ., Philadelphia, 858 S.

WignalL, P.B. (1994): Black Shales. - Clarendon Press, Oxford, 127 S.

WILDE, V \& FRANKENHÄUSER, H. (1998): The Middle Eocene plant taphocoenosis from Eckfeld (Eifel, Germany). - Rev. Palaeobot. Palynol., 101: 7-28.

Wilde, V., FrANKENHÄUSER, H. \& LUTZ, H. (1993): Algenreste aus den mitteleozänen Sedimenten des Eckfelder Maares bei Manderscheid in der Eifel. - Mainzer naturwiss. Archiv, 31: 127-148.

WILSON, M.V.H. (1993): Calibration of Eocene Varves at Horsefly, British Columbia, Canada, and Temporal Distribution of Specimens of the Eocene Fish Amyzon aggregatum WILSON. - Kaupia, 2: 2738.

WISE, S.W. \& KELTS, K.R. (1972): Inferred diagenetic history of a weakly silicified deep sea chalk. Trans. Gulf-Coast Assoc. Geol. Soc., 22: 177-203. 
WUTTKE, M. (1988): Erhaltung - Lösung - Umbau. Zum Verhalten biogener Stoffe bei der Fossilisation. In: SCHAAL, S. \& ZIEGLER, W. [Hrsg.]: Messel - Ein Schaufenster in die Geschichte der Erde und des Lebens. - Kramer, Frankfurt a.M., 265-275.

WUTTKE, M. \& RADTKE, G. (1993): Agglutinierende Mikrobenmatten im Profundal des mitteleozänen Eckfelder Maar-Sees bei Manderscheid/Eifel (Bundesrepublik Deutschland). - Mainzer naturwiss. Archiv, 31: 115-126.

ZimmeRLe, W. (1995): Petroleum Sedimentology. - In: BeCKMAnN, H. [ed.]: Geology of Petroleum, vol. 8. Enke, Stuttgart, $413 \mathrm{~S}$.

ZINK, K. \& PÜTTMANN, W. (1994): Biomarker-Analytik an ausgewählten Proben aus der Fossilfundstätte Eckfeld. - In: NeUfFER, F.O. et al. [Hrsg.]: Fossillagerstätte Eckfelder Maar. - Mainzer naturwiss. Archiv, Beiheft 16: 103-124.

ZIPPI, P.A. (1998): Freshwater algae from the Mattagami Formation (Albian), Ontario: Paleoecology, botanical affinities, and systematic taxonomy. - Micropaleontology, 44, suppl. no. 1: 1-78.

ZOLITSCHKA, B. (1993): Palaeoecological implications from the sedimentary record of a subtropical maar lake (Eocene Eckfelder Maar; Germany). - In: NEGENDANK, J. F. W. \& ZoLITSCHKA, B. [eds.]: Paleolimnology of European Maar Lakes. - Lekture Notes in Earth Sciences, 49: 477-484.

ZOLITSCHKA, B. (1998): Paläoklimatische Bedeutung Laminierter Sedimente. - Relief, Boden, Paläoklima, 13: 1-176.

ZsÓTÉR, M. (1997): Petrographische und geochemische Charakterisierung vulkanogener Gesteine (Tuffe und Alkalibasalte) des mitteleozänen Eckfelder Maares und zweier benachbarter Vulkanbauten (SWEifel, Deutschland). - Mainzer naturwiss. Archiv, 35: 61-82. 



\section{Dank}

Diese Arbeit entstand auf Anregung und unter der Betreuung von Prof. W. RIEGEL (Geowiss. Zentrum, Universität Göttingen). Herr Prof. J. PAUL (Geowiss. Zentrum, Universität Göttingen) übernahm freundlicherweise das Korreferat. Beiden danke ich für ihr stetes Interesse am Fortgang der Untersuchungen und die vielen anregenden Diskussionen.

Mein aufrichtiger Dank gilt weiterhin:

- Herrn M. SosNiTZA (Geowiss. Zentrum, Universität Göttingen) für sein Engagement und seinen Einfallsreichtum bei der Proben-Präparation.

- Herrn Dr. T. HEINRICHS (Geowiss. Zentrum, Universität Göttingen) für die Unterstützung am REM.

- allen Beteiligten an dem Gemeinschaftsprojekt "Bohrung Eckfelder Maar 1996", insbesondere Herrn Dr. H. LUTZ (Naturhist. Museum Mainz/Landessammlung für Naturkunde Rheinl.-Pfalz), Frau Dr. G. GRUBER (Hessisches Landesmuseum Darmstadt), Herrn Dr. V. WILDE und Frau Dr. B. NICKEL (beide Forsch.-Inst. Senckenberg Frankfurt/M), Herrn Dr. J. MINGRAM (GeoForschungsZentrum Potsdam), Herrn Dr. A. BECHTEL (Montanuniversität Leoben und Universität Bonn) und Herrn Dipl.-Geol. M. SABEL (Universität Bonn), Herrn Dr. C. FISCHER (Universität Jena) und Herrn Dipl.-Geol. W. SCHILLER (Universität Frankfurt/M). Ihre Anregungen, Hinweise und kritischen Bemerkungen gingen auf verschiedene Weise in diese Arbeit ein.

- den Gutachtern der Deutschen Forschungsgemeinschaft für die positive Beurteilung des Projektes Ri 154/23 "Organische Petrologie und Mikrofazies der mitteleozänen Seesedimente des Eckfelder Maares".

- Herrn Dipl.-Geol. A. QUAST (Geowiss. Zentrum, Universität Göttingen) für die freundschaftliche Zusammenarbeit sowie Frau Dipl.-Geol. C. HÜHNE, Frau Dipl.-Geol. M. PACHE, Herrn Dipl.-Geol. St. Delecat und Herrn Dr. St. STEINMETZ (alle Geowiss. Zentrum, Universität Göttingen) für die konstruktiven Kaffeepausen.

- meinen Eltern für die vielfältige Unterstützung.

Frau Christine HoLz danke ich aus unzähligen Gründen, in erster Linie aber für ihre Nachsicht. 



\section{Lebenslauf}

Geboren am 14. Mai 1969 in Bremerhaven

Staatsangehörigkeit: deutsch

\section{Hochschulausbildung und wissenschaftlicher Werdegang}

10/1990 - 10/1996 Studium der Geowissenschaften am Institut und Museum für Geologie und Paläontologie (IMGP) der Georg-August-Universität in Göttingen

12/1992 - 05/1993 Wissenschaftliche Hilfskraft am IMGP der Universität Göttingen

01/1997-07/1998 Examinierte wissenschaftliche Hilfskraft am IMGP der Universität Göttingen

08/1998-01/2003 Promotion am Geowissenschaftlichen Zentrum der Universität Göttingen (GZG) im Rahmen des von der DFG geförderten Forschungsprojektes Organische Petrologie und Mikrofazies der mitteleozänen Seesedimente des Eckfelder Maares (Südwesteifel) unter der Betreuung von Prof. Dr. W. Riegel

10/1999-02/2003 Studium der Wirtschaftswissenschaften an der Georg-AugustUniversität in Göttingen

seit $07 / 2002$

Beratender Geologe des von der DFG geförderten Sub-SaharaPaleogene-Projects der Universitäten Greifswald und Dar-es-Salaam (Tansania) 